\title{
Facile Fabrication of Cu-N-C Catalyst with Atomically Dispersed Unsaturated Cu-N2 Active Sites for Highly Efficient and Selective Glaser-Hay Coupling
}

Peng Ren, ${ }^{1,2}$ Qinglin Li, ${ }^{1,2}$ Tao Song, ${ }^{1}$ Yong Yang ${ }^{1 *}$

${ }^{1}$ CAS Key Laboratory of Bio-Based Materials, Qingdao Institute of Bioenergy and

Bioprocess Technology, Chinese Academy of Sciences, Qingdao 266101, China

${ }^{2}$ University of Chinese Academy of Sciences, Sino-Danish College, Beijing 100049, China

*Corresponding author

E-mail: yangyong@qibebt.ac.cn 


\section{Content}

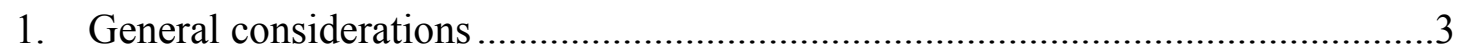

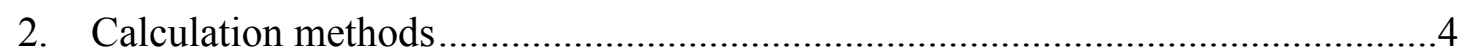

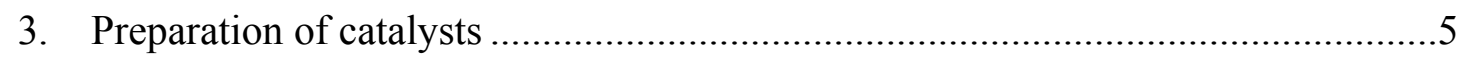

4. General procedures for the synthesis of (un)symmetrical 1,3-diynes.....................5

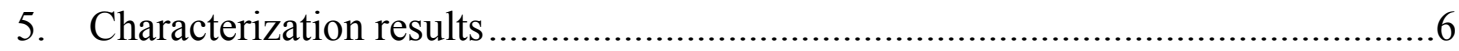

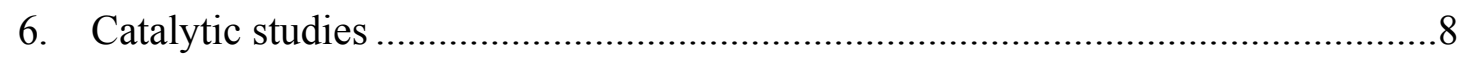

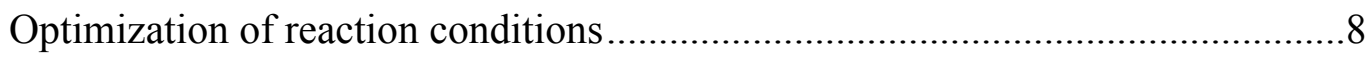

Substrate scopes for the synthesis of symmetrical 1,3-diynes ............................13

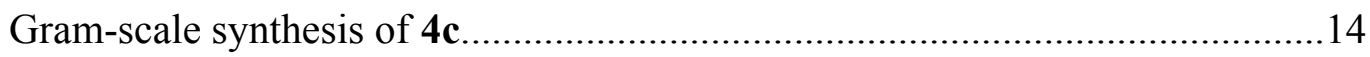

Recyclability of the catalyst $\mathrm{Cu}_{1} / \mathrm{NC}-800$ for the benchmark reaction................14

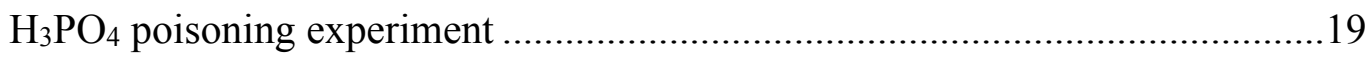

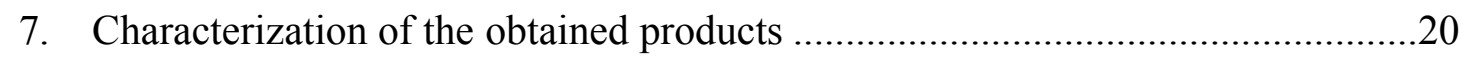

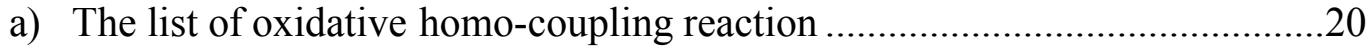

b) The list of oxidative cross-coupling reaction ............................................27

c) ${ }^{1} \mathrm{H}$ and ${ }^{13} \mathrm{C}$ NMR spectra of products of oxidative homo-coupling ................38

d) ${ }^{1} \mathrm{H}$ and ${ }^{13} \mathrm{C}$ NMR spectra of products of oxidative cross-coupling ................64

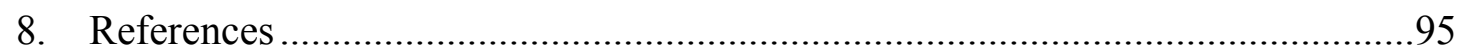




\section{General considerations}

Unless otherwise noted, all reagents were purchased commercially from Sigma-Aldrich, or Aladdin and used as received without further purification. The fresh bamboo shoots were obtained from Anhui Taiping Test Centre, International Centre for Bamboo and Rattan, Anhui Province, China. All operations were carried out in an argon atmosphere using glovebox and Schlenk techniques unless otherwise specified. The X-ray diffraction (XRD) patterns of all the catalysts were obtained on a Bruker D8 Advance X-ray diffraction diffractometer equipped with $\mathrm{Cu}$ Ka radiation $(\lambda=1.5147 \AA)$. The morphology of catalysts was examined by an H-7600 transmission electron microscopy (TEM), a Tecnai G2 F30 high-resolution TEM (HRTEM) and a FEI Tecnai G2 F20 scanning transmission electron microscopy (STEM). Nitrogen adsorption-desorption data were obtained on a Micromeritics ASAP 2020 static volumetric sorption analyzer. The specific surface area of the samples was calculated by the BrunauerEmmet-Teller (BET) method. The micropore volume was calculated by t-plot method. The pore size distributions were determined by non-local density functional theory (NLDFT). The X-ray photoelectron spectroscopy (XPS) data was collected on an ESCALAB 250Xi (Thermo Scientific, UK) instrument equipped with a monochromatized Al Ka line source. All the binding energies obtained were calibrated based on the $\mathrm{C} 1 \mathrm{~s}$ peak at $284.8 \mathrm{eV}$. The elemental composition analysis of the catalysts was conducted on Vario El elemental analyzer. Ion Chromatography was conducted on a Thermo Scientific Dionex ICS-5000 equipped with CS12 column with methanesulfonic acid $(20 \mathrm{mM})$ as an eluent. Raman spectra were obtained on a Horiba Jobin Yvon LabRAM HR800 Raman spectrometer system using a $532 \mathrm{~nm}$ wavelength laser at room temperature. Inductively coupled plasma atomic emission spectroscopy (ICPAES) was conducted on a PerkinElmer Optima 5300 DV instrument. X-ray absorption spectroscopy (XAS) measurements were carried out at room temperature on the $1 \mathrm{~W} 1 \mathrm{~B}$ beamline at BSRF (Beijing Synchrotron Radiation Facility). Samples were pelletized as disks of $6 \mathrm{~mm}$ diameter using paraffin as a binder, while the copper phthalocyanine $(\mathrm{CuPc}), \mathrm{Cu}_{2} \mathrm{O}$, $\mathrm{CuO}$ was mixed with $\mathrm{BN}$ powder. Transmission-mode $\mathrm{Cu}$ K-edge X-ray absorption spectra were collected for all samples at room temperature and the energy was calibrated by the absorption edge of $\mathrm{Cu}$ foil. The XAFS data were analyzed using IFEFFIT. The XAFS raw data 
were background subtracted, normalized and Fourier transformed by standard procedure within the ATHENA program. Gas chromatography analysis was performed on an Agilent HP-7890 instrument with a flame ionization detector (FID) and an HP-5MS capillary column (30 m, 0.25 mm i.d., $0.25 \mu \mathrm{m}$ film thicknesses) using helium as the carrier gas. Gas chromatography-mass spectrometry analysis was carried out on an Agilent HP-7890 instrument with an Agilent HP5975 with triple-axis detector and HP-5 capillary column using helium carrier gas. NMR spectra were from a Bruker DRX-400, or DRX-600, instrument and calibrated using residual nondeuterated solvent $\left(\mathrm{CDCl}_{3}: \delta_{\mathrm{H}}=7.26 \mathrm{ppm}, \delta_{\mathrm{C}}=77.16 \mathrm{ppm} ; \mathrm{DMSO}-d_{6}, \delta_{\mathrm{H}}=2.50 \mathrm{ppm}, \delta_{\mathrm{C}}=\right.$ $39.60 \mathrm{ppm}$ ) as an internal reference. High-resolution mass data were recorded on Bruke Maxis UHR TOF mass spectrometers in ESI mode.

\section{Calculation methods}

Density functional theory (DFT) calculations were performed with the Vienna ab-initio simulation package (VASP) ${ }^{1}$. In our calculations, the spin-polarized projector augmented wave (PAW) methods ${ }^{2}$ were applied together with the Perdew-Burke-Ernzerhof $(\mathrm{PBE})^{3}$ electron exchange correlation functional of the generalized gradient approximation (GGA) ${ }^{4}$. The energy cut-off of the wave function in the plane-wave basis was set to $400 \mathrm{eV}$, the calculations were carried out until the maximum force upon each relaxed atom was less than $0.05 \mathrm{eV} \AA^{-1}$.

In order to investigate the mechanisms of oxidative cross-coupling of terminal alkynes on the catalyst $\mathrm{Cu}_{1} / \mathrm{NC}-800$, 3-methoxyprop-1-yne and hex-5-ynenitrile were chosen as two model terminal alkynes for the calculation. The following models including $\mathrm{Cu}-\mathrm{N}_{4}, \mathrm{Cu}-\mathrm{N}_{3}, \mathrm{Cu}-\mathrm{N}_{2}$ and graphene were set up in a $(12.8 \times 12.3) \AA$ rectangle supercell of the graphene plane were investigated. The vacuum thickness between the graphene layers was set to $20 \AA$ to avoid interlayer interactions. The Brillouin zone was sampled by a $2 \times 2 \times 1 \mathrm{k}$-point grid with the Monkhorst-Pack scheme for structure optimization. The model of $\mathrm{Cu}-\mathrm{N}_{2}$ was set in a supercell of a graphene ribbon with armchair edges, consisting of 3 benzene rings, and the edge $\mathrm{C}$ atoms saturated with $\mathrm{H}$ atoms. The vacuum thickness in the directions perpendicular and parallel to the ribbon plane was set to about $20 \AA$. In addition, a $4 \times 4$ surface cell was used to construct a four-layer $\mathrm{Cu}(111)$ slab, the top three layers of the $\mathrm{Cu}(111)$ slab was allowed to relax. The Brillouin-zone integration was performed along with a $2 \times 2 \times 1$ Monkhorst-Pack grid for the different surface slabs. 
The adsorption energy ( $\left.\mathrm{E}_{\text {ads }}\right)$ was computed as below,

$$
E_{\text {ads }}=E_{\text {total }}-E_{\text {substrate }}-E_{\text {gas-phase adsorbate }}
$$

Equation 1

where $E_{\text {total }}$ is the total energy calculated for the adsorption system, $E_{\text {substrate }}$ is the calculated energy of the clean substrate and $E_{\text {gas-phase adsorbate }}$ is the calculated energy of the gas-phase molecule.

\section{Preparation of catalysts}

The catalyst $\mathrm{Cu}_{1} / \mathrm{NC}-800$ was prepared via a facile tandem hydrothermal-pyrolysis process. Typically, fresh bamboo shoots were first cut into small slices, dried and ground into powder followed by the hydrothermal carbonization (HTC) process in a Teflon-inner stainless-steel autoclave with deionized water at $180^{\circ} \mathrm{C}$. In this step, the bamboo shoots were converted into biochar. The resulting brown biochar was filtered, washed thoroughly with deionized water and dried under vacuum at room temperature. Next, the obtained solids were homogeneously mixed with copper nitrate solution at $60{ }^{\circ} \mathrm{C}$ for $2 \mathrm{~h}$ followed by drying and pyrolysis at $800^{\circ} \mathrm{C}$ under a $\mathrm{N}_{2}$ atmosphere for $2 \mathrm{~h}$ with a heating rate of $5^{\circ} \mathrm{C} \min ^{-1}$. The obtained catalyst was denoted as $\mathrm{Cu}_{1} / \mathrm{NC}-800$. For comparison, the catalyst $\mathrm{Cu} / \mathrm{AC}-800$ using activated carbon as the support instead under otherwise identical preparation procedure.

\section{General procedures for the synthesis of (un)symmetrical 1,3-diynes.}

A $25 \mathrm{~mL}$ glass tube was charged with a magnetic stirring bar, alkyne1 (0.13 mmol), alkyne2 (0.26 mmol), $\mathrm{Cu}_{1} / \mathrm{CN}-800$ (20 mg, 1.5 or $3 \mathrm{~mol} \%$ of $\mathrm{Cu}$ ), a mixture solvent of MeCN and water $(4 \mathrm{~mL}, 1 / 1, \mathrm{v} / \mathrm{v})$. The reaction was stirred for $18 \mathrm{~h}$ at $100^{\circ} \mathrm{C}$ under atmospheric air. After completion of the reaction, the reaction mixture was cooled to room temperature, the organic phase was analyzed by GC using trans-stilbene as an internal standard to measure the conversion and selectivity. The products were purified by flash column chromatography on silica gel using appropriate eluents and structurally confirmed by NMR. 


\section{Characterization results}
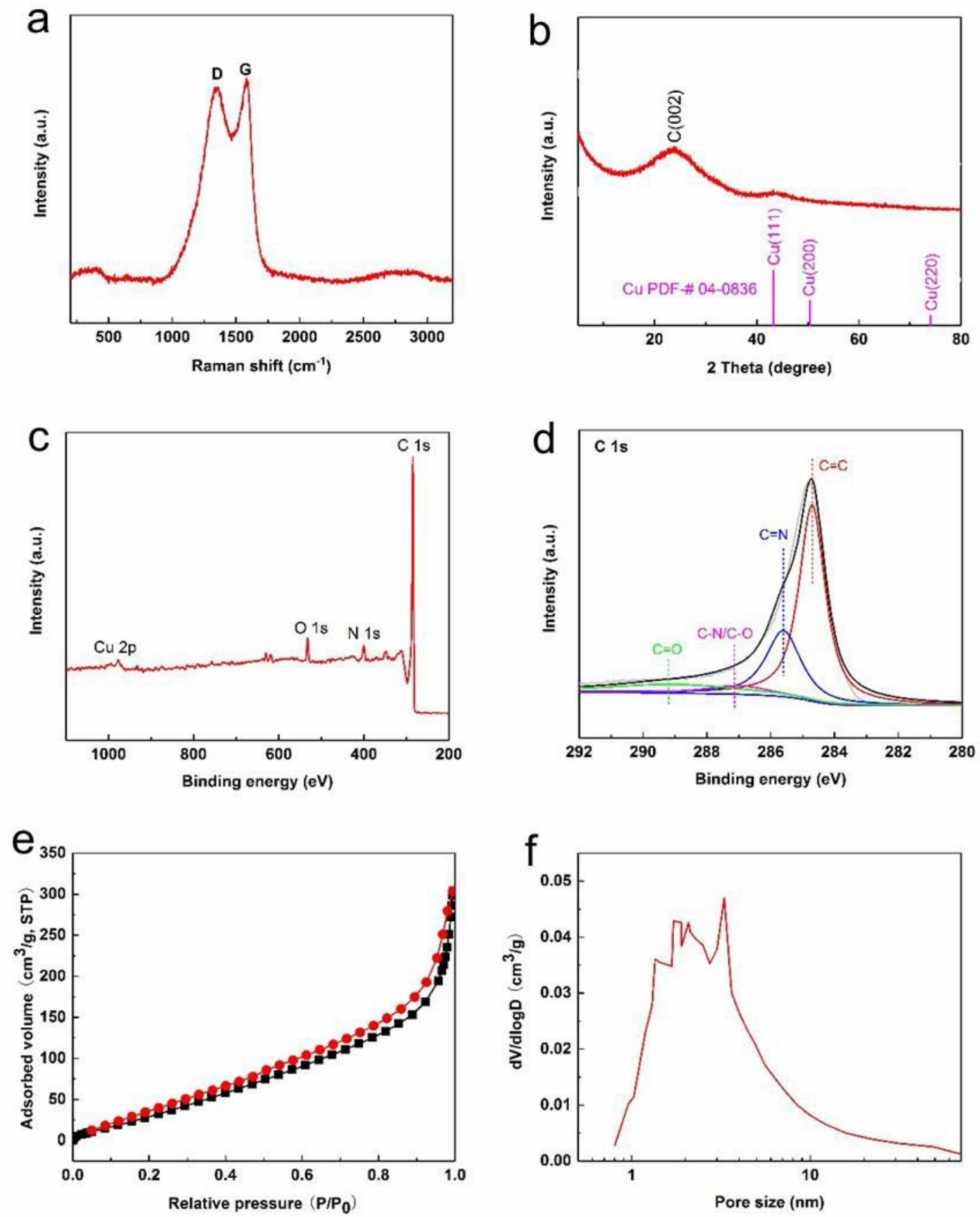

Figure S1. Characterization of the catalyst $\mathrm{Cu}_{1} / \mathrm{NC}-800$ : (a) Raman spectrum, (b) XRD diffraction with $\mathrm{Cu}$ reference, (c) XPS survey spectrum, (d) C 1s XPS, (e) $\mathrm{N}_{2}$ adsorptiondesorption isotherms, and (f) Nonlocal Density Functional Theory (NLDFT) adsorption pore size distribution. 


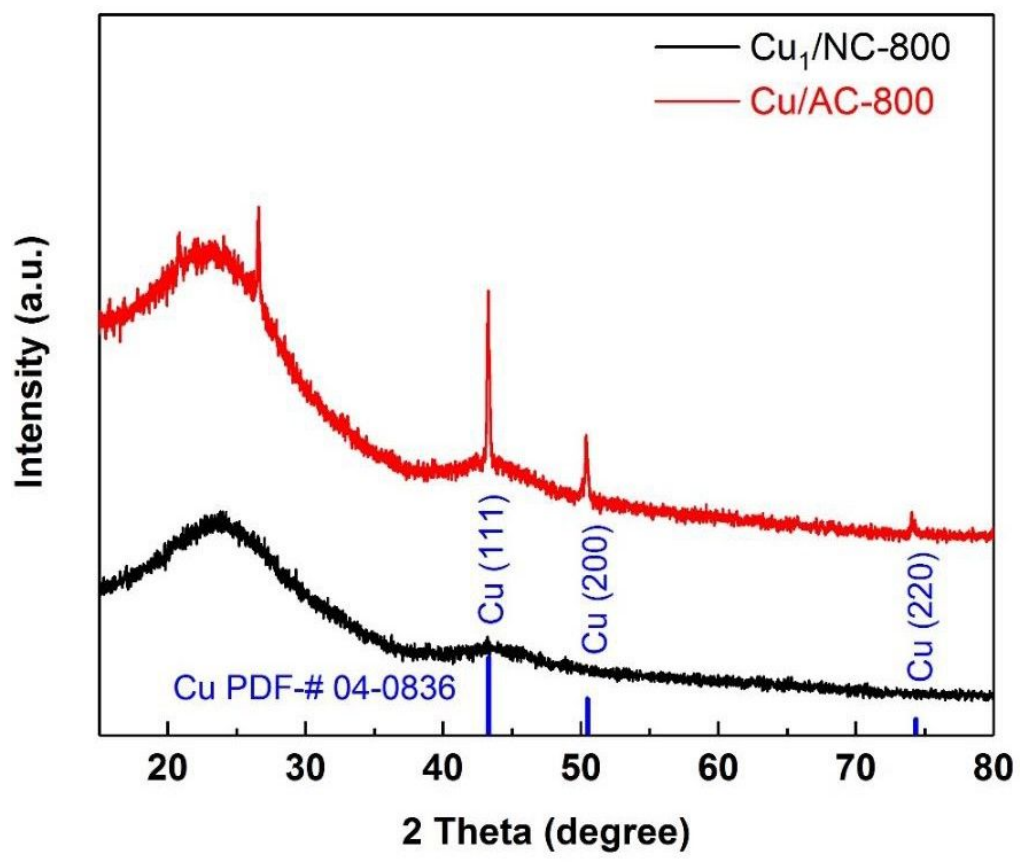

Figure S2. XRD pattern for the catalysts $\mathrm{Cu}_{1} / \mathrm{NC}-800$ and $\mathrm{Cu} / \mathrm{AC}-800$.

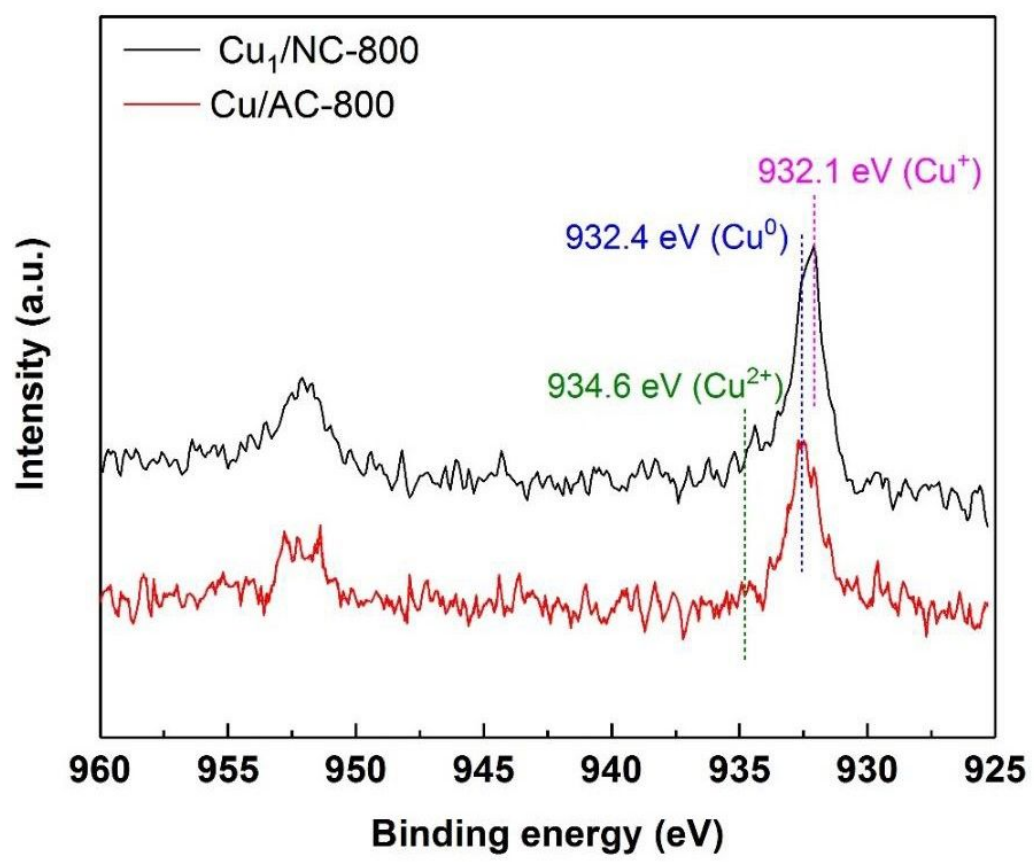

Figure S3. $\mathrm{Cu} 2 \mathrm{p}$ XPS spectra for the catalysts $\mathrm{Cu}_{1} / \mathrm{NC}-800$ and $\mathrm{Cu} / \mathrm{AC}-800$. 
Table S1. Chemical composition and texture properties of the catalyst $\mathrm{Cu}_{1} / \mathrm{NC}-800$ and $\mathrm{Cu} / \mathrm{AC}-$ 800.

\begin{tabular}{lcccccc}
\hline Sample & $\mathrm{Cu}$ & \multicolumn{3}{c}{ Elemental analysis } & \multicolumn{2}{c}{ BET analysis } \\
\cline { 3 - 7 } & $\begin{array}{c}\text { Content } \\
(\mathrm{wt} \%)^{\mathrm{a}}\end{array}$ & $\begin{array}{c}\mathrm{C} \\
(\mathrm{wt} \%)\end{array}$ & $\begin{array}{c}\mathrm{O} \\
(\mathrm{wt} \%)\end{array}$ & $\begin{array}{c}\mathrm{N} \\
(\mathrm{wt} \%)\end{array}$ & $\begin{array}{c}\text { S } \mathrm{BET} \\
\left(\mathrm{m}^{2} \mathrm{~g}^{-1}\right)^{\mathrm{b}}\end{array}$ & $\begin{array}{c}\text { Pore volume } \\
\left(\mathrm{cm}^{3} \mathrm{~g}^{-1}\right)^{\mathrm{c}}\end{array}$ \\
\hline $\mathrm{Cu} / \mathrm{CN}-800$ & 1.28 & 78.34 & 10.49 & 5.44 & 150.5 & 0.423 \\
$\mathrm{Cu} / \mathrm{AC}-800$ & 1.31 & 92.28 & 6.47 & 0.03 & 1129.4 & 0.913 \\
\hline
\end{tabular}

${ }^{\text {a } D e t e r m i n e d ~ b y ~ I C P-A E S . ~}{ }^{b}$ Specific surface areas were determined by the BET multipoint

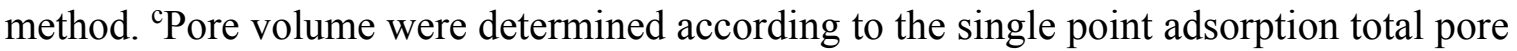
volume of pores less than $187.2160 \mathrm{~nm}$ width at $\mathrm{P} / \mathrm{Po}=0.99$.

Table S2. Curve-fitting analysis of EXAFS spectrum of the catalyst $\mathrm{Cu}_{1} / \mathrm{NC}-800$ and $\mathrm{CuPc}$ as a reference. ${ }^{\text {a] }}$

\begin{tabular}{lccccc}
\hline Sample & Shell & $\mathbf{C N}{ }^{[\mathbf{b}]}$ & $\mathbf{R}\left(\AA^{\left[{ }^{[\mathbf{c}]}\right.}\right.$ & $\begin{array}{c}\Delta \boldsymbol{\sigma}^{\mathbf{2}[\mathbf{d}]} \\
\left(\mathbf{\AA}^{\left.\mathbf{2} \times \mathbf{1 0}^{-\mathbf{3}}\right)}\right.\end{array}$ & $\begin{array}{c}\Delta \boldsymbol{E} \\
(\mathbf{e V})^{[\mathrm{e}]}\end{array}$ \\
\hline \multirow{2}{*}{$\mathrm{Cu}_{1} / \mathrm{NC}-800$} & $\mathrm{Cu}-\mathrm{Cu}$ & - & - & 0.009 & $2.2 \pm 1.2$ \\
\cline { 2 - 4 } & $\mathrm{Cu}-\mathrm{N}$ & $2.2 \pm 0.3$ & $1.89 \pm 0.03$ & & \\
\hline $\mathrm{CuPc}$ & $\mathrm{Cu}-\mathrm{N}$ & $3.9 \pm 0.4$ & $1.94 \pm 0.02$ & 0.004 & $8.2 \pm 1.6$ \\
\hline
\end{tabular}

[a] Fourier transform and Fourier-filtering regions were limited, where $\Delta \mathrm{k}=3.0 \sim 14.0 \AA^{-1}$ and $\Delta \mathrm{r}=1.7 \sim 3.2 \AA$, respectively. [b] Coordination number. [c] Bond distance between absorber and backscatter atoms. [d] The Debye-Waller factor (DW). [e] The inner potential correction accounts for the difference in the inner potential between the sample and reference.

\section{Catalytic studies}

\section{Optimization of reaction conditions}

Table S3. Optimization of reaction conditions for the homocoupling. ${ }^{a}$

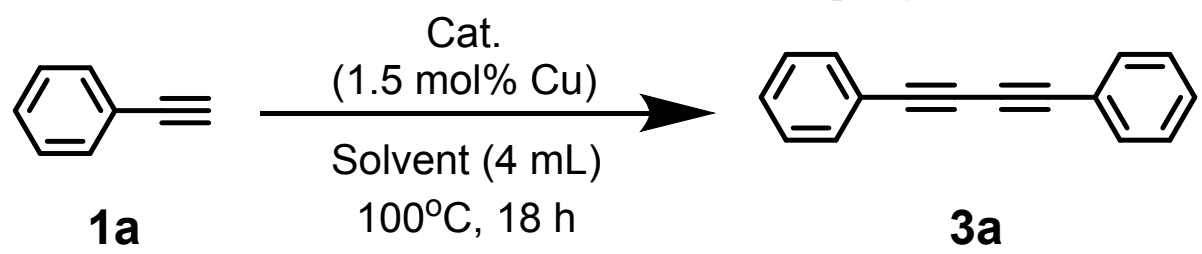




\begin{tabular}{|c|c|c|c|}
\hline Entry & $\begin{array}{c}\text { Catalyst } \\
(1.5 \mathrm{~mol} \% \mathrm{Cu})\end{array}$ & Solvent & Yield $(\%)^{b}$ \\
\hline 1 & $\mathrm{Cu}_{1} / \mathrm{NC}-800$ & Acetone & 0 \\
\hline 2 & $\mathrm{Cu}_{1} / \mathrm{NC}-800$ & Toluene & 0 \\
\hline 3 & $\mathrm{Cu}_{1} / \mathrm{NC}-800$ & $\mathrm{CH}_{2} \mathrm{Cl}_{2}$ & 0 \\
\hline 4 & $\mathrm{Cu}_{1} / \mathrm{NC}-800$ & Ethanol & trace \\
\hline 5 & $\mathrm{Cu}_{1} / \mathrm{NC}-800$ & Ethyl acetate & 0 \\
\hline 6 & $\mathrm{Cu}_{1} / \mathrm{NC}-800$ & Isopropanol & trace \\
\hline 7 & $\mathrm{Cu}_{1} / \mathrm{NC}-800$ & 1,4-dioxane & trace \\
\hline 8 & $\mathrm{Cu}_{1} / \mathrm{NC}-800$ & $\mathrm{MeCN}$ & 10 \\
\hline 9 & $\mathrm{Cu}_{1} / \mathrm{NC}-800$ & Water & trace \\
\hline 10 & $\mathrm{Cu}_{1} / \mathrm{NC}-800$ & EtOH/water (4:1) & 18 \\
\hline 11 & $\mathrm{Cu}_{1} / \mathrm{NC}-800$ & IPA/water (4:1) & 21 \\
\hline 12 & $\mathrm{Cu}_{1} / \mathrm{NC}-800$ & $\mathrm{MeCN} /$ water $(4: 1)$ & 37 \\
\hline 13 & $\mathrm{Cu}_{1} / \mathrm{NC}-800$ & $\mathrm{MeCN} /$ water $(1: 1)$ & 98 \\
\hline $14^{\mathrm{c}}$ & $\mathrm{Cu}_{1} / \mathrm{NC}-800$ & $\mathrm{MeCN} /$ water $(1: 1)$ & 0 \\
\hline 15 & $\mathrm{Cu} / \mathrm{AC}-800$ & $\mathrm{MeCN} /$ water $(1: 1)$ & 18 \\
\hline 16 & NC-800 & $\mathrm{MeCN} /$ water(1:1) & trace \\
\hline 17 & $\mathrm{Cu}$ & MeCN/water(1:1) & 12 \\
\hline 18 & $\mathrm{Cu}\left(\mathrm{NO}_{3}\right)_{2}$ & MeCN/water(1:1) & 15 \\
\hline 19 & $\mathrm{Cu}_{2} \mathrm{O}$ & MeCN/water(1:1) & 8 \\
\hline 20 & $\mathrm{CuO}$ & MeCN/water(1:1) & 0 \\
\hline 21 & $\mathrm{CuPc}$ & MeCN/water(1:1) & 0 \\
\hline 22 & Blank & MeCN/water(1:1) & 0 \\
\hline
\end{tabular}

${ }^{a}$ Reaction conditions: phenylacetylene (1a, $\left.0.2 \mathrm{mmol}\right)$, catalyst $(1.5 \mathrm{~mol} \%$ of $\mathrm{Cu})$, solvent (4 $\mathrm{mL}), 100^{\circ} \mathrm{C}$, under atmospheric air. ${ }^{b}$ Determined by GC using trans-stilbene as an internal standard sample and confirmed with its corresponding authentic sample. ${ }^{\text {cUnder }}$ argon atmosphere. 
A set of reaction parameters including solvent and different $\mathrm{Cu}$ sources were intensively screened to optimize the reaction conditions. As shown in Table S3, those common organic solvents, such as acetone, toluene, $\mathrm{CH}_{2} \mathrm{Cl}_{2}$, ethyl acetate, ethanol, iropropanol, and 1,4-dioxane are not suitable for the reaction with no reactivity (entries 1-7), while $\mathrm{MeCN}$ gives $10 \% \mathrm{GC}$ yield of the desired product (entry 8). Addition of a certain volume of water into the ethanol, iropropanol or $\mathrm{MeCN}$ as solvent significantly facilitates the reaction (entries 10-12), especially for the reaction using $\mathrm{MeCN}$ as solvent (entry 12). Further studies show that increasing the volume of water in the solvent, nearly quantitative conversion was achieved under otherwise identical conditions (entry 13). However, the reaction didn't proceed when it was performed under argon atmosphere, indicating air as the oxidant is necessary for the success of the reaction (entry 14). For comparison, other $\mathrm{Cu}$ sources, e.g., $\mathrm{Cu}$ powder, $\mathrm{Cu}_{2} \mathrm{O}, \mathrm{Cu}\left(\mathrm{NO}_{3}\right)_{2}, \mathrm{CuO}, \mathrm{CuPc}$, were screened (entries 17-20) and they exhibited low or no reactivity. This finding was further verified by the catalyst $\mathrm{Cu} / \mathrm{AC}-800$ with obvious formation of the metallic $\mathrm{Cu}$ nanoparticles on carbon (Figure S3 and S4). In this case, only 18\% GC yield of the desired product was observed, which is a larger 5 times lower than that of the catalyst $\mathrm{Cu}_{1} / \mathrm{NC}-800$, highlighting the distinct advantage of the atomically dispersed $\mathrm{Cu}$ for the reaction. In addition, the reaction didn't occur using NC-800 as a catalyst or in the absence of a catalyst (entries 16 and 21), indicating the critical role the catalyst $\mathrm{Cu}_{1} / \mathrm{NC}-800$.

Table S4. Optimization of reaction conditions for the heterocoupling. ${ }^{\mathrm{a}}$

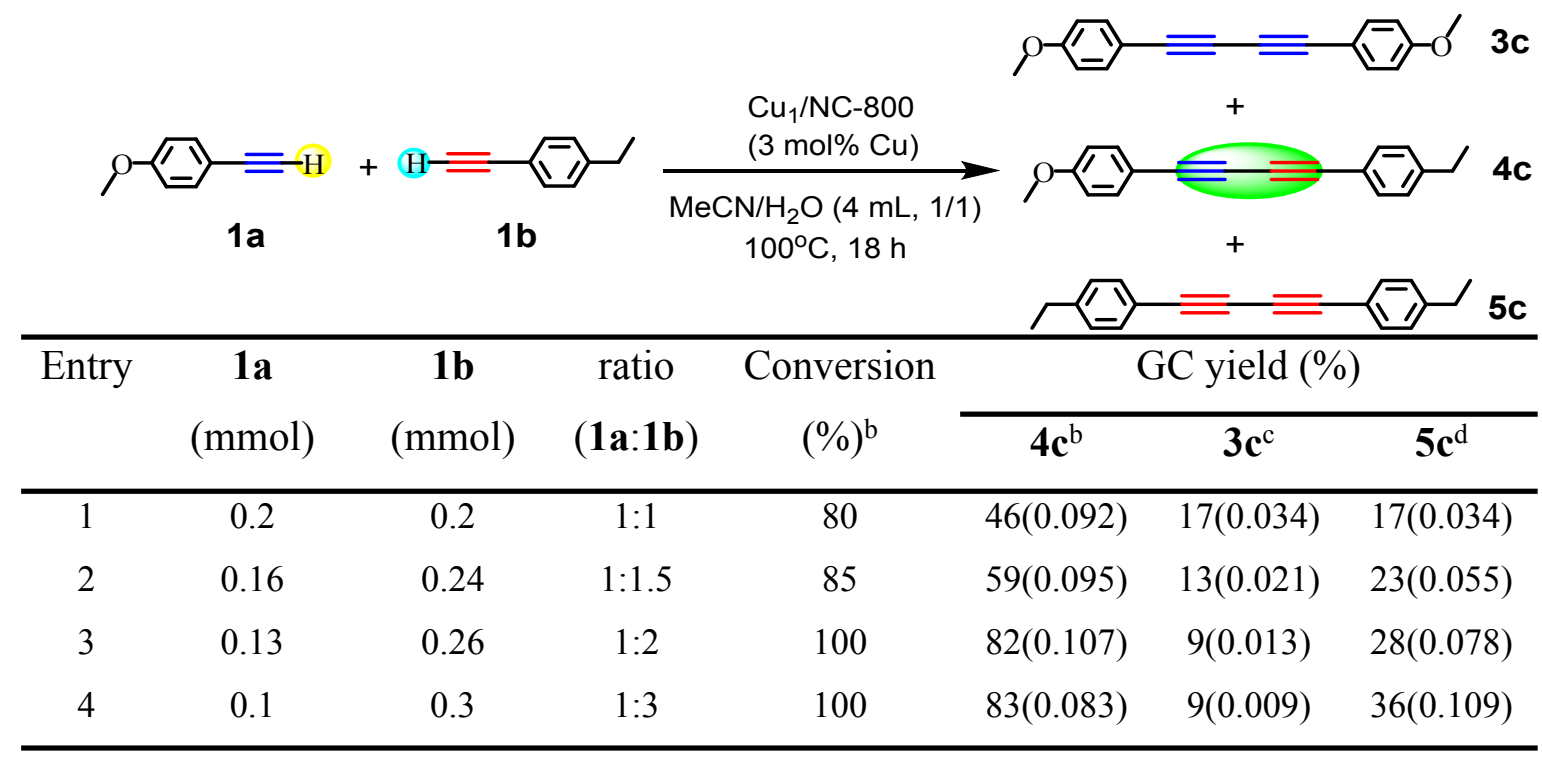

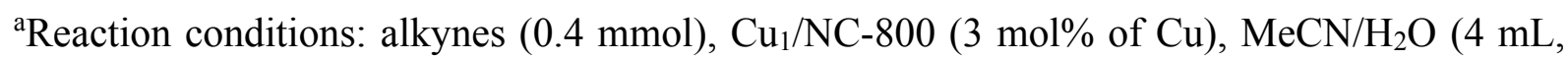


$1 / 1, \mathrm{v} / \mathrm{v}), 100^{\circ} \mathrm{C}$, under atmospheric air. ${ }^{b}$ Determined by GC using trans-stilbene as an internal standard sample based on 1a. ${ }^{\mathrm{b}} \mathrm{GC}$ yield based on 1a; The number in the parentheses are amounts of $\mathbf{3 c}(\mathrm{mmol}){ }^{\mathrm{c}}{ }^{\mathrm{GC}}$ yield based on 1a; The number in the parentheses are amounts of $\mathbf{4 c}(\mathrm{mmol}) .{ }^{\mathrm{d}} \mathrm{GC}$ yield based on $\mathbf{1 b}$; The number in the parentheses are amounts of $\mathbf{5 c}(\mathrm{mmol})$.

With the optimal conditions for the homocoupling of terminal alkynes in hand, we further explored it for synthesis of unsymmetrical 1,3-diynes via the oxidative cross-coupling of two different terminal alkynes. Basically, the ratio of two terminal alkynes played a critical role on the selectivity to the desired unsymmetrical 1,3-diynes, and a large excess of one terminal alkyne is required in most cases catalysed by copper catalysts. As such, we intensively studied the influence of the ratio of two different terminal alkynes on the selectivity. As shown in Table $\mathrm{S} 4$, we found that a maximum yield to $3 \mathbf{c}$ could be accomplished with the ratio of $1 / 2$ for the two alkynes (entry 3$)$. 
Table S5. Comparison of the catalytic activity for the synthesis of unsymmetrical 1,3-diynes from two terminal alkynes over different catalysts.

\begin{tabular}{|c|c|c|c|c|c|c|c|c|c|c|}
\hline \multirow[t]{2}{*}{ Entry } & \multicolumn{7}{|c|}{ Reaction conditions } & \multirow{2}{*}{$\begin{array}{l}\text { Yield } \\
(\%)\end{array}$} & \multirow[t]{2}{*}{ TON } & \multirow[t]{2}{*}{ Ref. } \\
\hline & $\begin{array}{c}\mathrm{R}^{1-} \\
\text { alkyne } \\
(\mathrm{mmol})\end{array}$ & $\begin{array}{l}\text { Catalyst } \\
(\mathrm{mol} \%)\end{array}$ & $\begin{array}{c}\text { Ratio } \\
\left(\mathrm{R}^{2}-/ \mathrm{R}^{1}-\right. \\
\text { alkyne }))\end{array}$ & Base & $\begin{array}{l}\text { Ligand } \\
(\mathrm{mol} \%)\end{array}$ & Oxidant & Solvent & & & \\
\hline 2 & 0.2 & $\begin{array}{c}\operatorname{dppm}(\mathrm{AuBr})_{2} \\
(2.5)\end{array}$ & 1.3 & & $\begin{array}{c}\text { phenanthroline } \\
\text { (10) }\end{array}$ & $\begin{array}{l}\mathrm{PhI}(\mathrm{OAc})_{2} \\
(2 \text { equiv. })\end{array}$ & $\begin{array}{c}\mathrm{CH}_{3} \mathrm{CN} / 1,4 \text {-dioxane } \\
(0.6 \mathrm{~mL} / 0.2 \mathrm{~mL})\end{array}$ & 85 & 34 & 2 \\
\hline 4 & 0.42 & $\begin{array}{c}\mathrm{Cu} / \mathrm{Cu}_{2} \mathrm{O} @ \mathrm{COF} \\
(0.0992)\end{array}$ & 1.2 & & $\begin{array}{l}\text { TMEDA } \\
(47.62)\end{array}$ & Air & $\begin{array}{c}\mathrm{CHCl}_{3} / 1,4 \text {-dioxane } \\
(0.3 \mathrm{~mL} / 0.1 \mathrm{~mL})\end{array}$ & 70 & 170 & 4 \\
\hline 5 & 0.3 & $\begin{array}{c}\mathrm{Au} @ \mathrm{NH}_{2}-\mathrm{SBA}-15 \\
(9.9)\end{array}$ & 2 & & $\begin{array}{c}1,10- \\
\text { phenanthroline } \\
(40)\end{array}$ & $\begin{array}{l}\mathrm{PhI}(\mathrm{OAc})_{2} \\
\text { (1.5 equiv.) }\end{array}$ & $\begin{array}{c}\text { DCM } \\
(4 \mathrm{~mL})\end{array}$ & 80 & 8.1 & 5 \\
\hline 6 & 0.16 & $\begin{array}{c}\text { Meso } \mathrm{Cu} / \mathrm{MnO}_{\mathrm{x}} \\
(18)\end{array}$ & 2 & & & Air & $\begin{array}{l}\text { Toluene } \\
(5 \mathrm{~mL})\end{array}$ & 50 & 2.7 & 6 \\
\hline
\end{tabular}


Table S6. Close Comparison of the catalytic activity for the synthesis of unsymmetrical 1,3-diynes from two terminal alkynes over different catalysts.

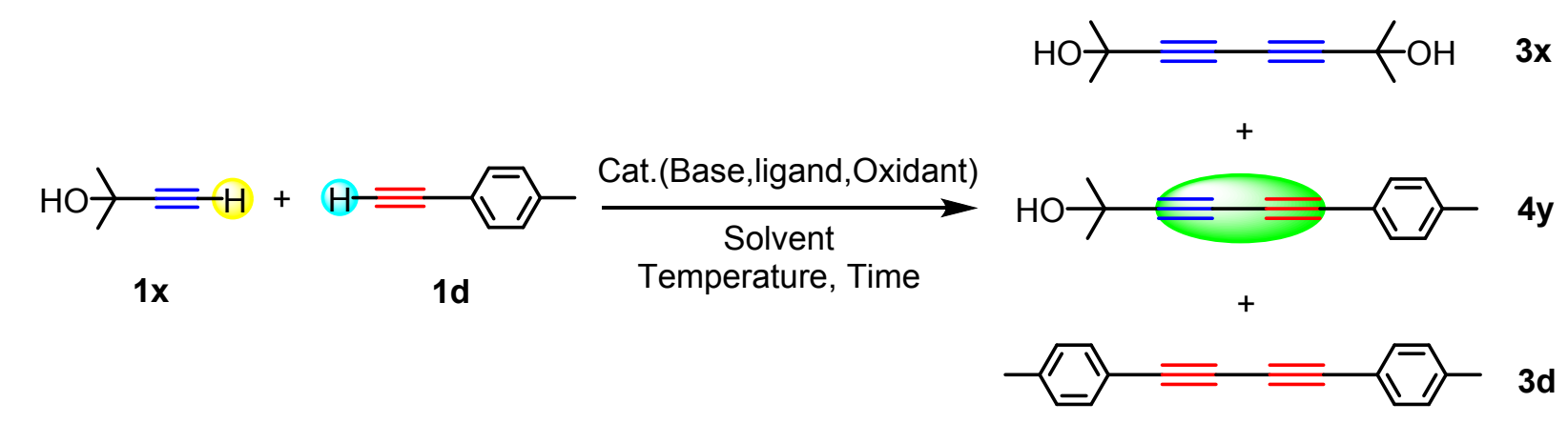

\begin{tabular}{|c|c|c|c|c|c|c|c|c|c|c|c|c|c|c|}
\hline \multirow[t]{2}{*}{$\overline{\text { Entry }}$} & \multicolumn{8}{|c|}{ Reaction conditions } & \multicolumn{3}{|c|}{ Yield $^{\mathrm{b}}(\%)$} & \multirow[b]{2}{*}{$4 y / 3 x / 3 d$} & \multirow[t]{2}{*}{ TON } & \multirow[t]{2}{*}{ Ref. } \\
\hline & $\begin{array}{c}\mathbf{1 x} \\
(\mathrm{mmol})\end{array}$ & $\begin{array}{l}\text { Catalyst } \\
(\mathrm{mol} \%)\end{array}$ & $\begin{array}{c}\text { Ratio } \\
(\mathbf{1 x} / \mathbf{1 d})\end{array}$ & $\begin{array}{c}\mathrm{T} \\
{ }^{\circ} \mathrm{C} \\
\end{array}$ & Base & $\begin{array}{l}\begin{array}{l}\text { Ligand } \\
(\mathrm{mol} \%)\end{array} \\
\end{array}$ & Oxidant & Solvent & $4 y$ & $3 x$ & $3 d$ & & & \\
\hline 1 & 0.2 & $\begin{array}{l}\text { Cu powder } \\
\text { (5) }\end{array}$ & 1.3 & 50 & - & $\begin{array}{l}\text { TMEDA } \\
\text { (20) }\end{array}$ & Air & $\begin{array}{c}\mathrm{CHCl}_{3} / 1,4- \\
\text { dioxane } \\
(0.3 \mathrm{~mL} / 0.1 \\
\mathrm{mL})\end{array}$ & 73 & 7.3 & 16.06 & $10 / 1 / 2.2$ & 14.6 & 1 \\
\hline 2 & 0.2 & $\begin{array}{c}\operatorname{dppm}(\mathrm{AuBr})_{2} \\
(2.5)\end{array}$ & 1.3 & 50 & - & $\begin{array}{c}\text { phenanthroline } \\
\text { (10) }\end{array}$ & $\begin{array}{l}\mathrm{PhI}(\mathrm{OAc})_{2} \\
(2 \text { equiv. })\end{array}$ & $\begin{array}{c}\mathrm{CH}_{3} \mathrm{CN} / 1,4- \\
\text { dioxane } \\
(0.6 \mathrm{~mL} / 0.2 \\
\mathrm{mL})\end{array}$ & 80 & - & - & - & 32 & 2 \\
\hline 3 & 0.13 & $\begin{array}{c}\mathrm{Cu}_{1} / \mathrm{NC}-800 \\
\text { (3) }\end{array}$ & 2 & 100 & - & - & Air & $\begin{array}{c}\text { MeCN/Water } \\
(2 \mathrm{~mL} / 2 \mathrm{~mL})\end{array}$ & 70 & 10 & 30 & $7 / 1 / 3$ & 23.3 & $\begin{array}{l}\text { This } \\
\text { work }\end{array}$ \\
\hline
\end{tabular}




\section{Ref.}

1. Su, L.; Dong, J.; Liu, L.; Sun, M.; Qiu, R.; Zhou, Y.; Yin, S. F., Copper Catalysis for Selective Heterocoupling of Terminal Alkynes. J. Am. Chem. Soc. 2016, 138, 12348. 2 Peng, H.; Xi, Y.; Ronaghi, N.; Dong, B.; Akhmedov, N. G.; Shi, X., Goldcatalyzed oxidative cross-coupling of terminal alkynes: selective synthesis of unsymmetrical 1,3-diynes. J. Am. Chem. Soc. 2014, 136, 13174.

3. $\mathrm{Xu}, \mathrm{H}$.; $\mathrm{Wu}, \mathrm{K}$;; Tian, J.; Zhu, L.; Yao, X., Recyclable $\mathrm{Cu} / \mathrm{C}_{3} \mathrm{~N}_{4}$ composite catalysed homo- and cross-coupling of terminal alkynes under mild conditions. Green Chem. 2018, 20, 793.

4. Chakraborty, D.; Nandi, S.; Mullangi, D.; Haldar, S.; Vinod, C. P.; Vaidhyanathan, $\mathrm{R}$., $\mathrm{Cu} / \mathrm{Cu}_{2} \mathrm{O}$ Nanoparticles Supported on a Phenol-Pyridyl COF as a Heterogeneous Catalyst for the Synthesis of Unsymmetrical Diynes via Glaser-Hay Coupling. ACS Appl. Mater. Inter. 2019, 11, 15670.

5. Vilhanová, B.; Václavík, J.; Artiglia, L.; Ranocchiari, M.; Togni, A.; van Bokhoven, J. A., Subnanometer Gold Clusters on Amino-Functionalized Silica: An Efficient Catalyst for the Synthesis of 1,3-Diynes by Oxidative Alkyne Coupling. ACS Catal. 2017, 7, 3414.

6. $\quad$ Biswas, S.; Mullick, K.; Chen, S.-Y.; Kriz, D. A.; Shakil, M. D.; Kuo, C.-H.; Angeles-Boza, A. M.; Rossi, A. R.; Suib, S. L., Mesoporous Copper/Manganese Oxide Catalyzed Coupling of Alkynes: Evidence for Synergistic Cooperative Catalysis. ACS Catal. 2016, 6, 5069.

7. Yin, W.; He, C.; Chen, M.; Zhang, H.; Lei, A, Nickel-Catalyzed Oxidative Coupling Reactions of Two Different Terminal Alkynes Using $\mathrm{O}_{2}$ as the Oxidant at Room Temperature: Facile Syntheses of Unsymmetric 1,3-Diynes. Org. Lett. 2009, 11, 709. 


\section{Gram-scale synthesis of $4 c$.}

A $100 \mathrm{~mL}$ round-bottom flask was charged with a magnetic stirring bar, 1-ethynyl-4methoxybenzene (1a), 1-ethyl-4-ethynylbenzene (1b), $\mathrm{Cu}_{1} / \mathrm{NC}-800(1.3 \mathrm{~mol} \%$ of $\mathrm{Cu})$, $\mathrm{MeCN} / \mathrm{H}_{2} \mathrm{O}(50 \mathrm{~mL})$. The reaction was stirred at preheated oil bath $\left(100^{\circ} \mathrm{C}\right)$ for $48 \mathrm{~h}$ under atmospheric air. After completion of the reaction, the reaction mixture was cooled to room temperature for workup. The product 3c was purified by flash column chromatography and structurally confirmed by NMR.

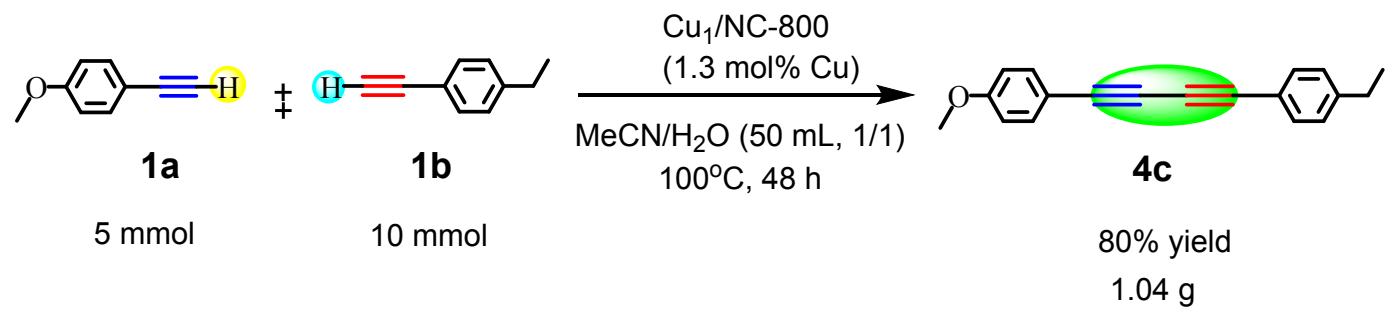

Scheme S1. Gram-scale synthesis of $\mathbf{3 c}$ under standard conditions.

\section{Recyclability of the catalyst $\mathrm{Cu}_{1} / \mathrm{NC}-800$ for the benchmark reaction.}

The benchmark reaction was chosen to investigate the recyclability of the catalyst $\mathrm{Cu}_{1} / \mathrm{NC}$ 800. A $25 \mathrm{~mL}$ reaction tube was charged with a magnetic stirring bar, $1 \mathbf{a}(0.13 \mathrm{mmol}), \mathbf{1 b}$ (0.26 mmol), $\mathrm{Cu}_{1} / \mathrm{NC}-800$ ( $3 \mathrm{~mol} \%$ of $\left.\mathrm{Cu}\right), \mathrm{MeCN} / \mathrm{H}_{2} \mathrm{O}(4 \mathrm{~mL})$. The reaction was stirred at preheated oil bath $\left(100^{\circ} \mathrm{C}\right)$ under atmospheric air for $18 \mathrm{~h}$. After completion of the reaction, the reaction mixture was cooled to room temperature, and the catalyst was carefully collected by washing with $\mathrm{MeCN}$ and drying under vacuum for next use. To check the stability of $\mathrm{Cu}$ species on the support, hot filtration experiment was performed. When the conversion of $1 \mathbf{a}$ approached approximately $40 \%$, the catalyst $\mathrm{Cu}_{1} / \mathrm{NC}-800$ was filtered immediately. The collected filtrate was further hold at $100^{\circ} \mathrm{C}$ oil bath for continuing the reaction. The reaction progress was monitored by GC. 

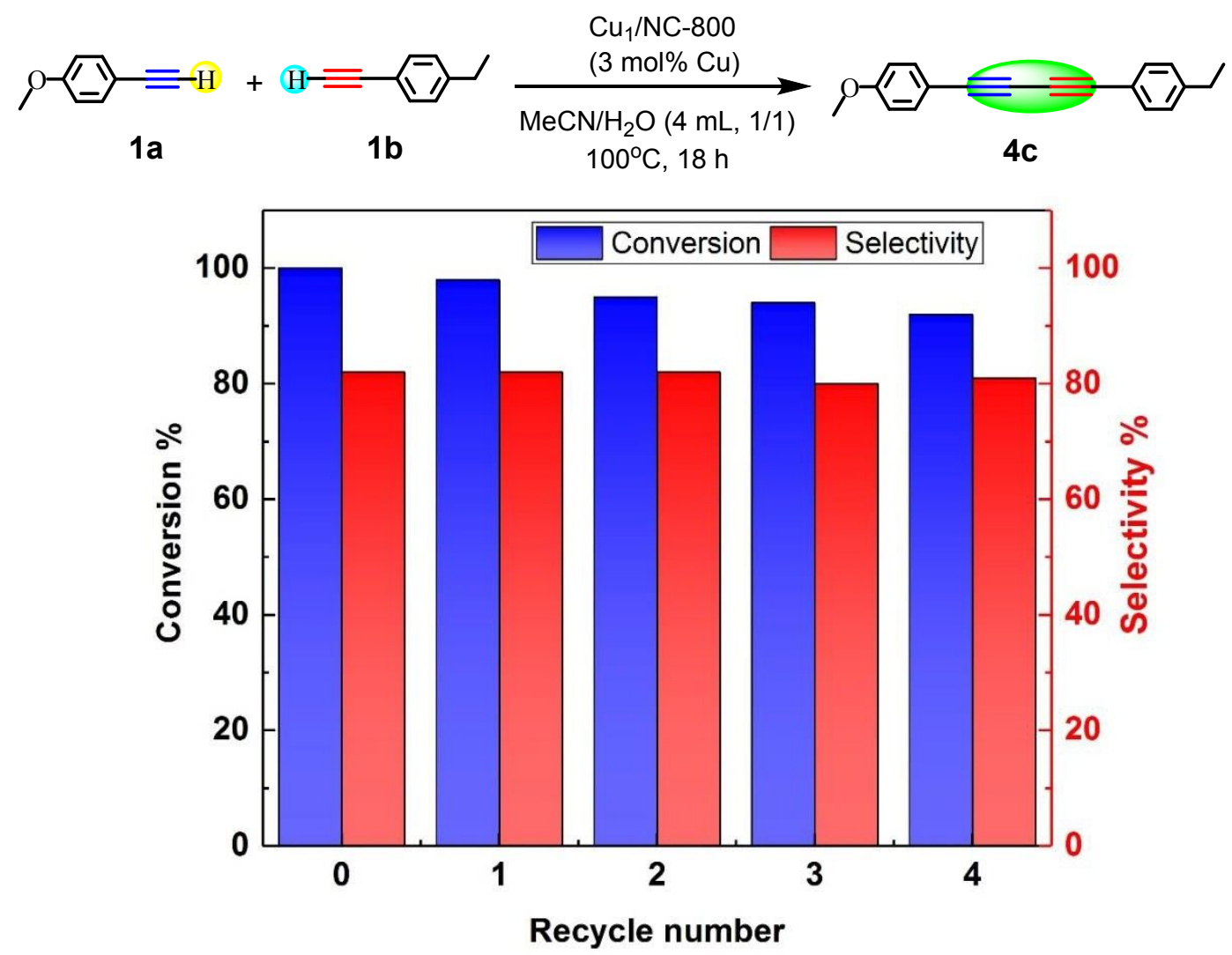

Figure S4. Recyclability of the catalyst $\mathrm{Cu}_{1} / \mathrm{NC}-800$ for the benchmark reaction under the standard conditions.

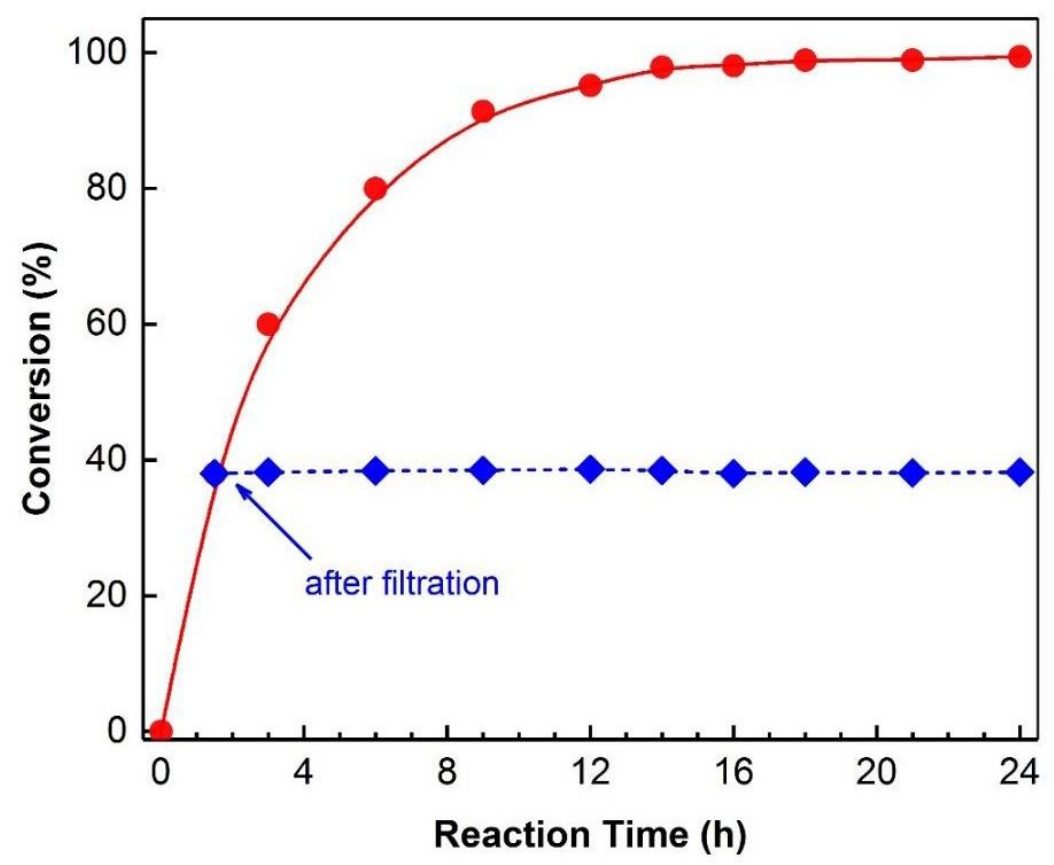

Figure S5. The hot filtration experiment of the benchmark reaction under the standard conditions. 


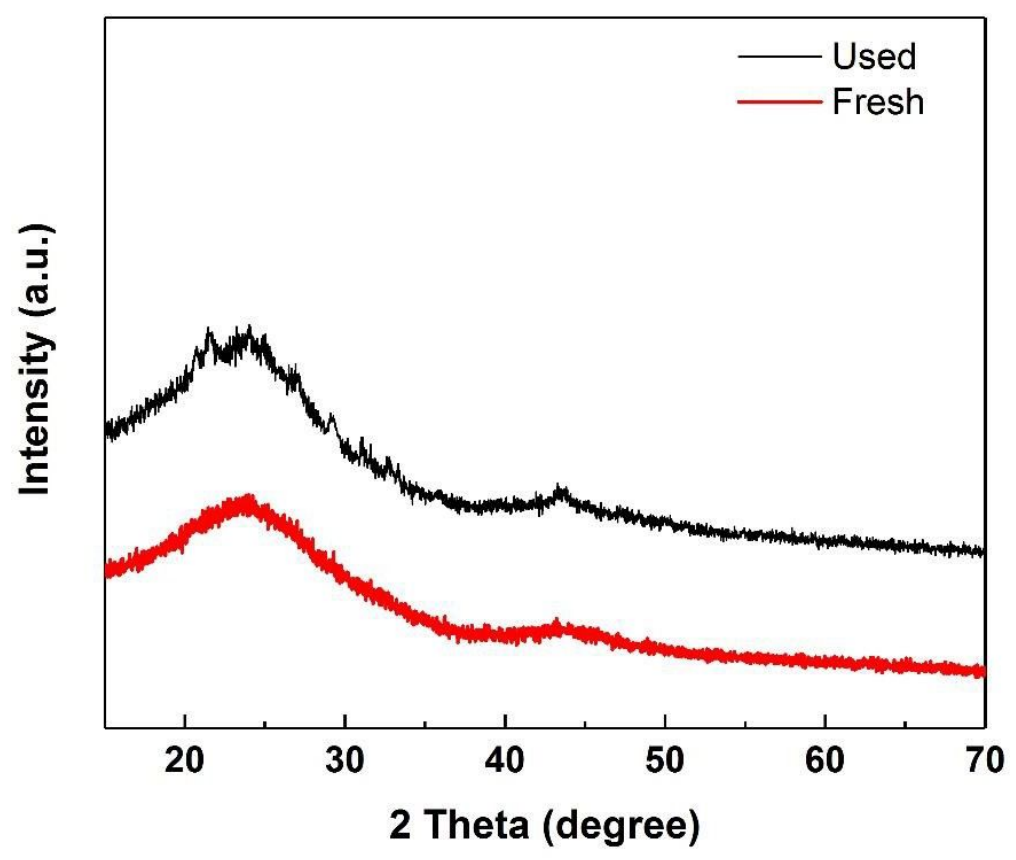

Figure S6. XRD pattern of the fresh and used catalyst $\mathrm{Cu}_{1} / \mathrm{NC}-800$.
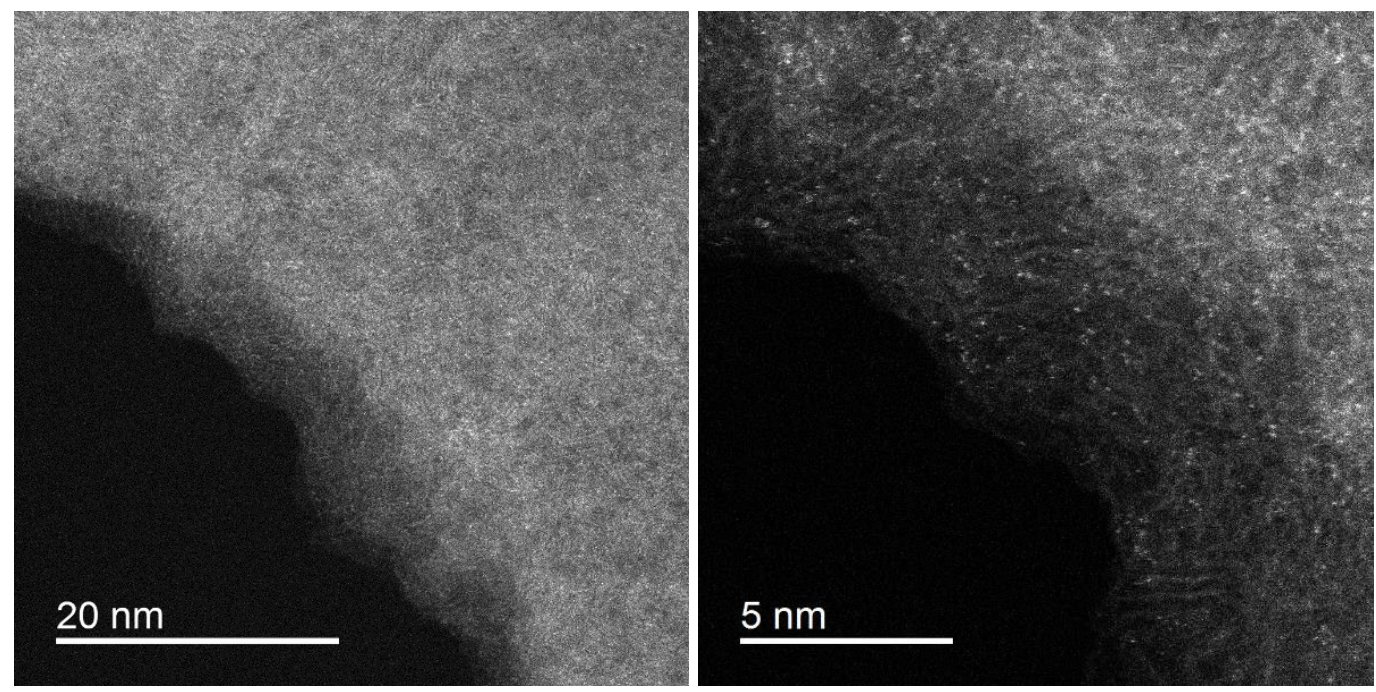

Figure S7. AC HAADF-STEM images of the used catalyst $\mathrm{Cu}_{1} / \mathrm{NC}-800$. 
IM1
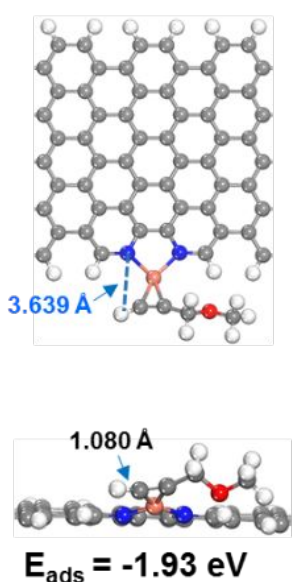

TS1
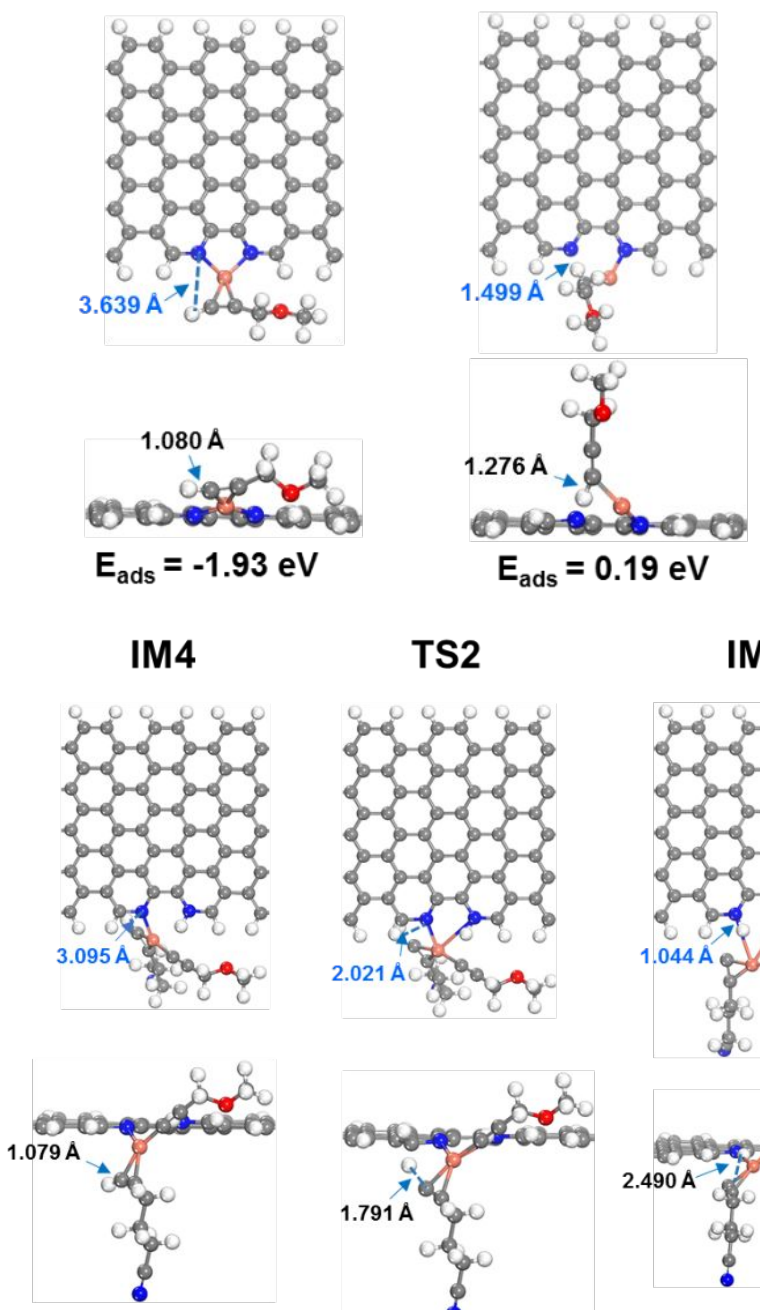

$E_{\text {ads }}=-1.64 \mathrm{eV}$
TS2

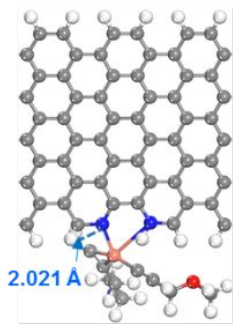

IM4
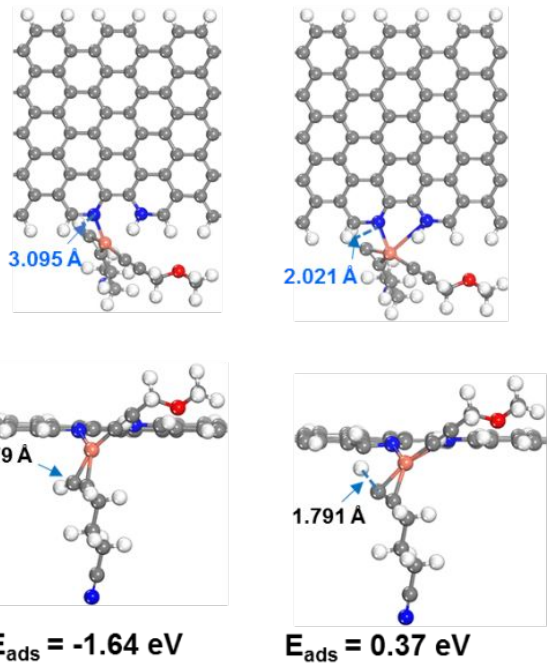

IM2
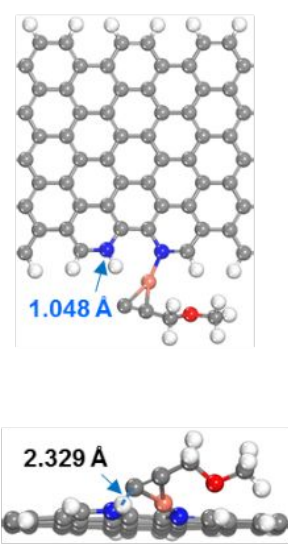

$E_{\text {ads }}=-0.36 \mathrm{eV}$

IM6
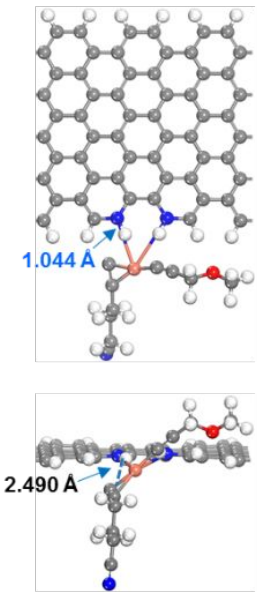

$E_{\text {ads }}=-0.37 \mathrm{eV}$
IM3
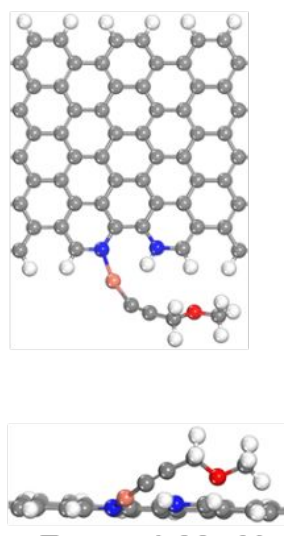

$E_{\text {ads }}=-1.00 \mathrm{eV}$

IM7

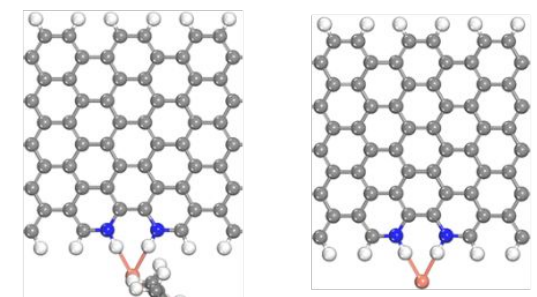

$0-0.00-00$

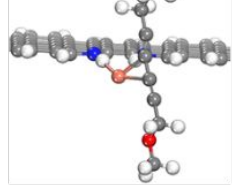

$E_{\text {ads }}=0.72 \mathrm{eV}$

$E_{\text {ads }}=0.66 \mathrm{eV}$

Figure S8. The energy changes of alkynes on the $\mathrm{Cu}-\mathrm{N}_{2}$ site in different states. 


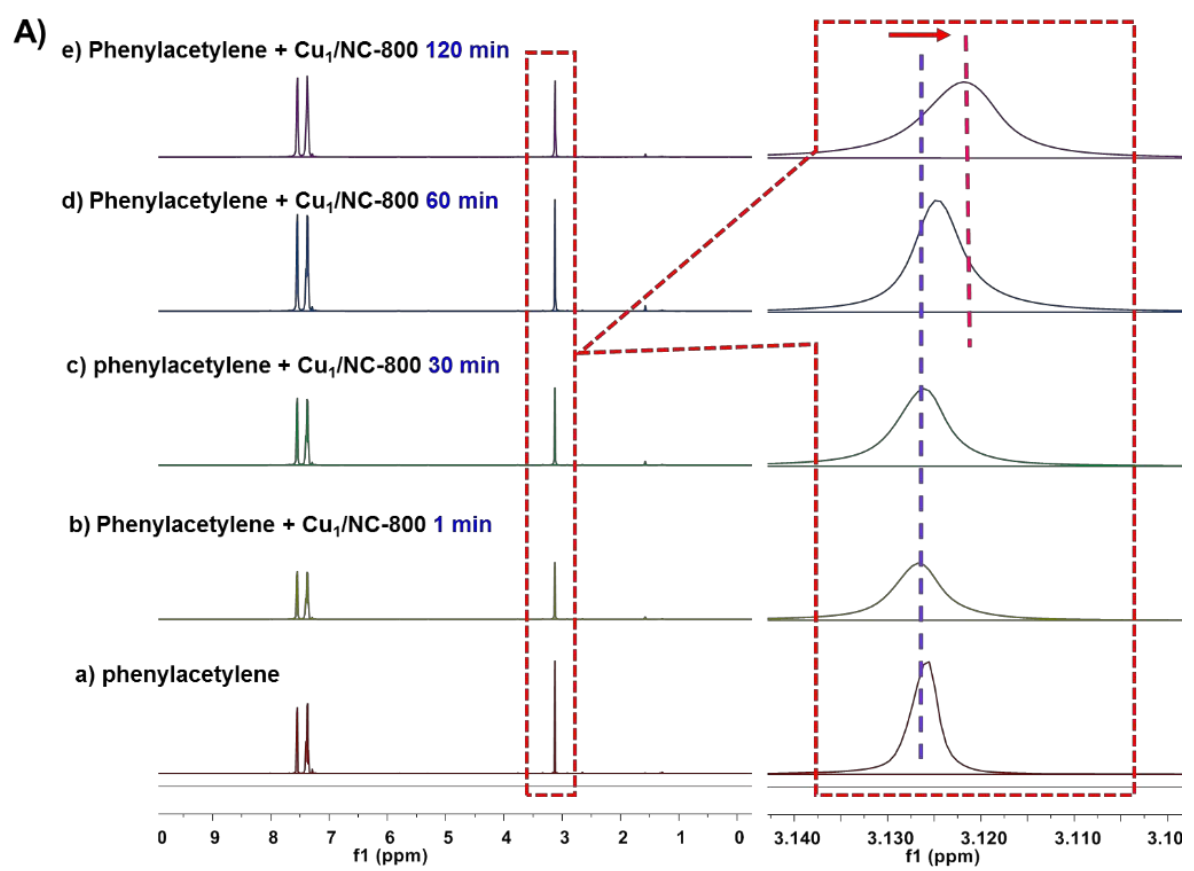

Figure S9. Variation of ${ }^{1} \mathrm{H}$ NMR spectroscopy of phenylacetylene as a function of time in $d$ $\mathrm{CDCl}_{3}$ containing the catalyst $\mathrm{Cu}_{1} / \mathrm{NC}-800$ under nitrogen at room temperature.

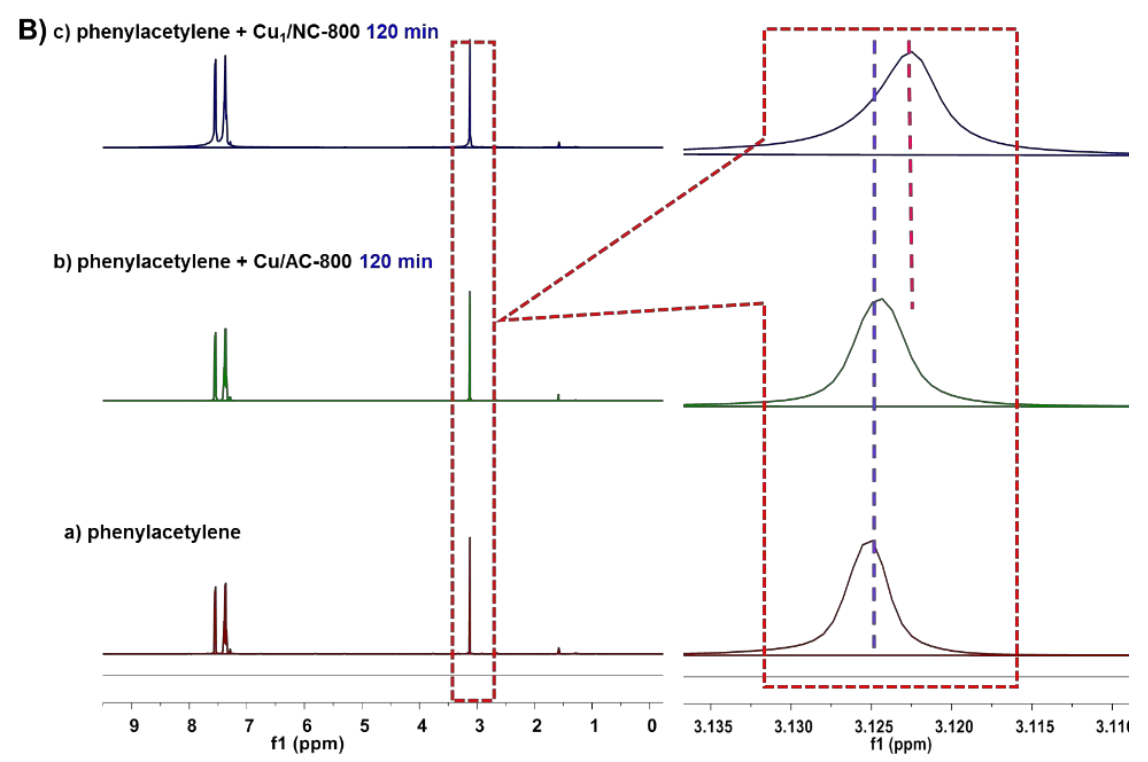

Figure S10. Comparison of ${ }^{1} \mathrm{H}$ NMR spectroscopy of phenylacetylene in $d$ - $\mathrm{CDCl}_{3}$ containing the catalyst $\mathrm{Cu}_{1} / \mathrm{NC}-800$ and $\mathrm{Cu} / \mathrm{AC}-800$ under nitrogen at room temperature. 


\section{$\mathrm{H}_{3} \mathrm{PO}_{4}$ poisoning experiment}

Before reaction, a given amount of $\mathrm{H}_{3} \mathrm{PO}_{4}$ was added into the mixed solvent of $\mathrm{CH}_{3} \mathrm{CN} / \mathrm{H}_{2} \mathrm{O}$ containing the catalyst $\mathrm{Cu}_{1} / \mathrm{NC}-800$. After stirring for $10 \mathrm{~min}$ at room temperature, two terminal alkynes (1a and 1b) were then injected into the solution and subsequently hold at preheated oil bath $\left(100^{\circ} \mathrm{C}\right)$ with stirring. After $18 \mathrm{~h}$, the reaction was analyzed by $\mathrm{GC}$ to determine the conversion and selectivity. The added amount of $\mathrm{H}_{3} \mathrm{PO}_{4}$ corresponds to alkyne 1a, for example, $0.025,0.05,0.1,0.3$, and 0.5 equivalents to $\mathbf{1 a}$.
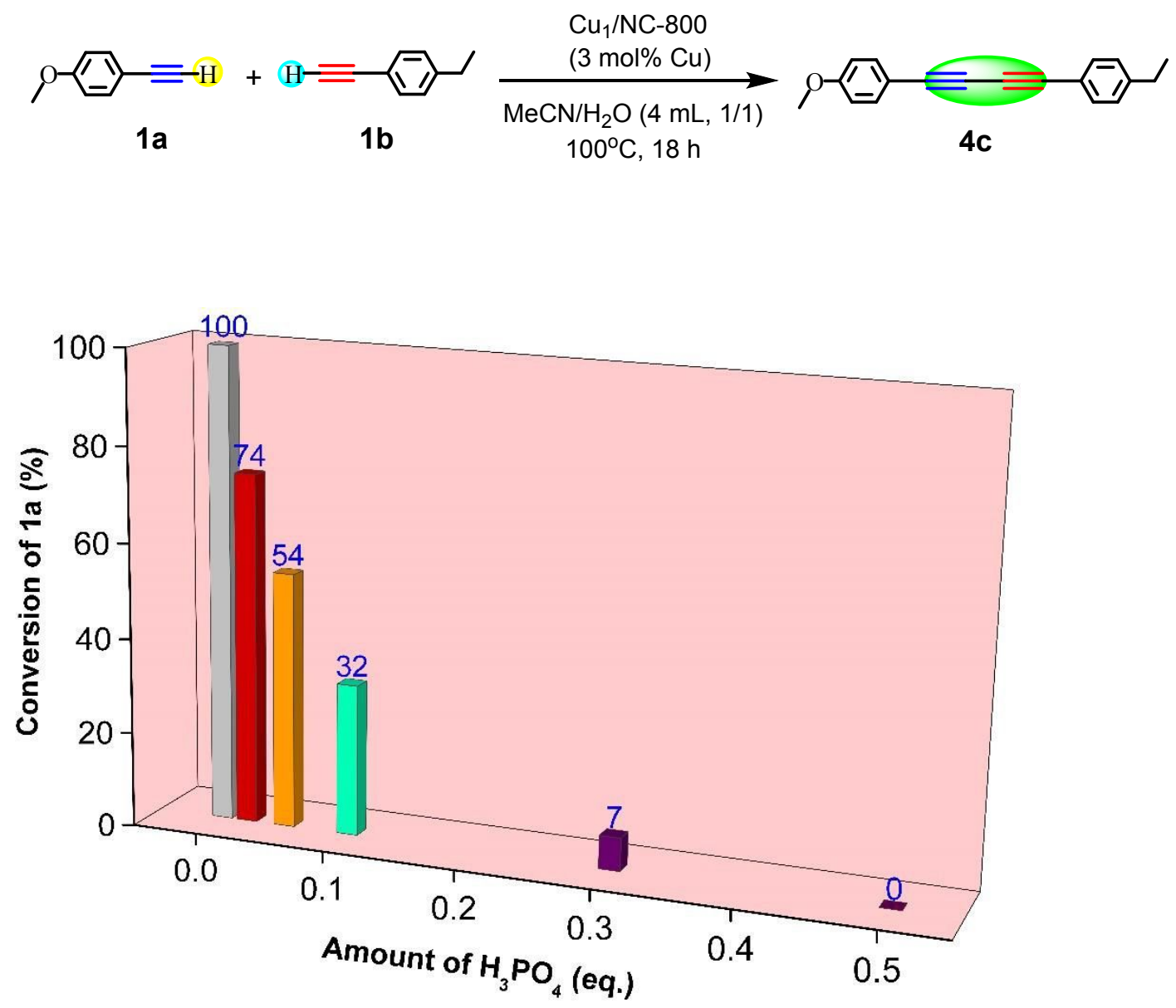

Figure S11. Result of $\mathrm{H}_{3} \mathrm{PO}_{4}$ poisoning experiment. 


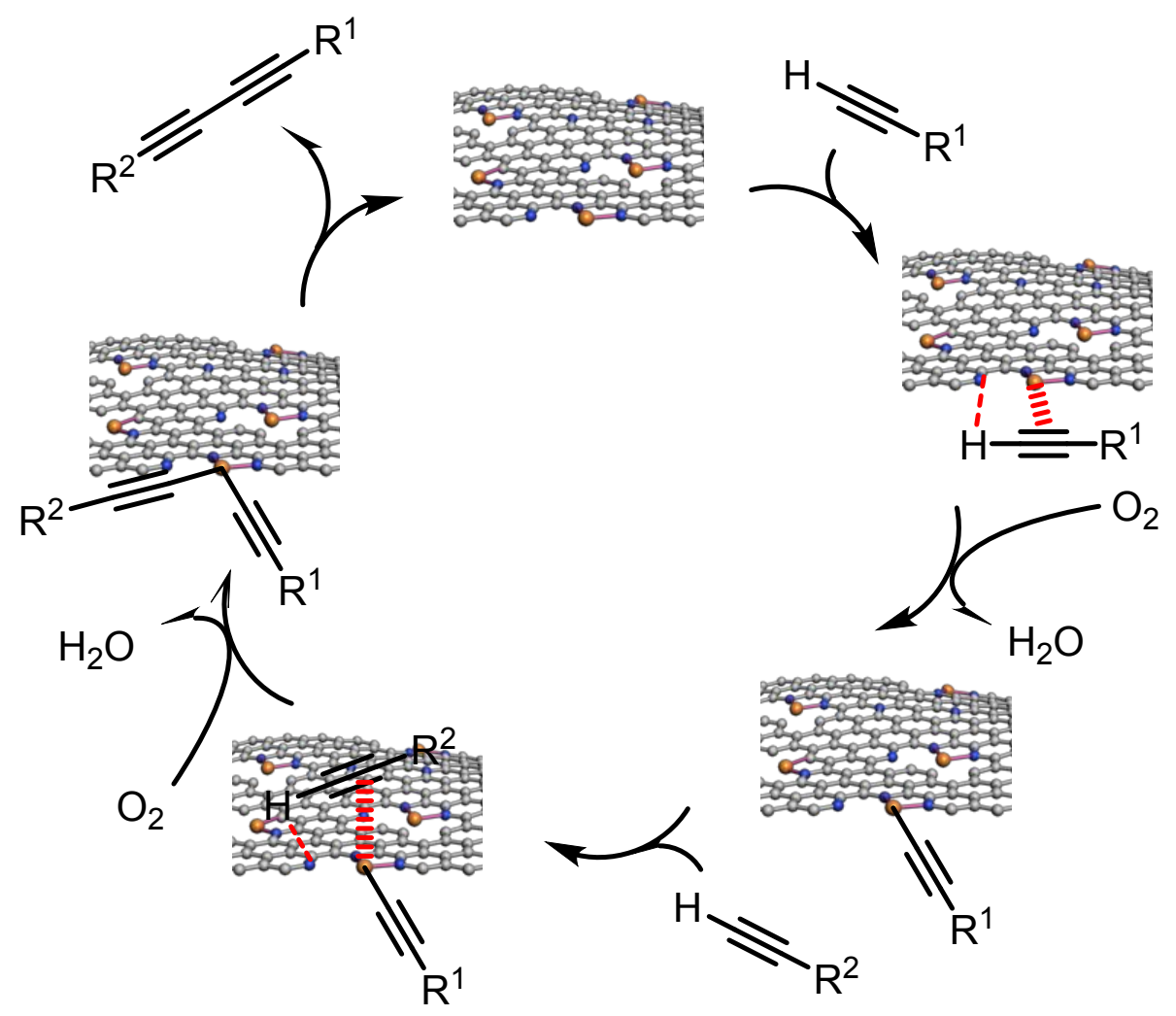

Figure S12. A proposed mechanism. 


\section{Characterization of the obtained products}

a) The list of oxidative homo-coupling reaction

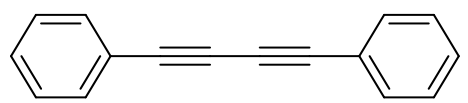

1,4-diphenylbuta-1,3-diyne (3a): Following general method. Ethynylbenzene (1a; $20.4 \mathrm{mg}$, $0.2 \mathrm{mmol})$ afforded the title compound as a white solid $(19.8 \mathrm{mg}, 98 \%)$ by using petroleum ether as the column eluent; ${ }^{1} \mathbf{H}$ NMR $\left(400 \mathrm{MHz}, \mathrm{CDCl}_{3}\right) \delta 7.66-7.48(\mathrm{~m}, 4 \mathrm{H}), 7.35$ (q, $J=7.2$, $6.5 \mathrm{~Hz}, 6 \mathrm{H}) .{ }^{13} \mathbf{C} \mathbf{N M R}\left(101 \mathrm{MHz}, \mathrm{CDCl}_{3}\right) \delta 132.52,129.23,128.46,121.81,81.58,73.94$. The spectroscopic data matched that previously report. ${ }^{5}$

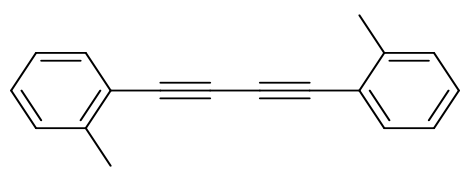

1,4-di-o-tolylbuta-1,3-diyne (3b): Following general method. 1-ethynyl-2-methylbenzene (1b; $23.2 \mathrm{mg}, 0.2 \mathrm{mmol})$ afforded the title compound as a white solid $(22.6 \mathrm{mg}, 98 \%)$ by using petroleum ether as the column eluent; ${ }^{1} \mathbf{H}$ NMR $\left(400 \mathrm{MHz}, \mathrm{CDCl}_{3}\right) \delta 7.43$ (dd, $J=7.6,1.3 \mathrm{~Hz}$, 2H), $7.20(\mathrm{dd}, J=7.5,1.4 \mathrm{~Hz}, 1 \mathrm{H}), 7.18-7.13(\mathrm{~m}, 3 \mathrm{H}), 7.08(\mathrm{td}, J=7.3,1.5 \mathrm{~Hz}, 2 \mathrm{H}), 2.42(\mathrm{~s}$, 6H). ${ }^{13} \mathrm{C}$ NMR $\left(101 \mathrm{MHz}, \mathrm{CDCl}_{3}\right) \delta 140.60,131.87,128.53,128.06,124.61,120.66,80.09$, 75.66, 19.72. The spectroscopic data matched that previously report. ${ }^{6}$

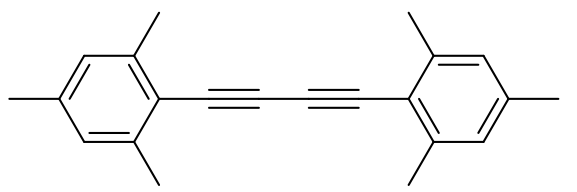

1,4-dimesitylbuta-1,3-diyne (3c): Following general method. 2-ethynyl-1,3,5trimethylbenzene (1c; $28.8 \mathrm{mg}, 0.2 \mathrm{mmol})$ afforded the title compound as a white solid (23.2 mg, $81 \%$ ) by using petroleum ether as the column eluent; ${ }^{1} \mathbf{H}$ NMR $\left(400 \mathrm{MHz}, \mathrm{CDCl}_{3}\right) \delta 6.89$ (s, 4H), 2.47 (s, 12H), 2.30 (s, 6H). ${ }^{13} \mathbf{C}$ NMR (151 MHz, $\left.\mathrm{CDCl}_{3}\right) \delta 141.72,138.57,127.72$, $118.94,80.59,80.51,21.44,21.01$. The spectroscopic data matched that previously report. ${ }^{7}$

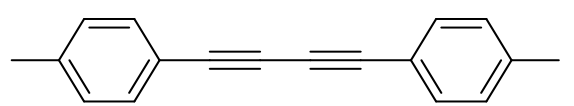

1,4-di-p-tolylbuta-1,3-diyne (3d): Following general method. 1-ethynyl-4-methylbenzene (1d; $23.2 \mathrm{mg}, 0.2 \mathrm{mmol}$ ) afforded the title compound as a white solid (22.3 $\mathrm{mg}, 97 \%)$ by using petroleum ether as the column eluent; ${ }^{1} \mathbf{H}$ NMR $\left(400 \mathrm{MHz}, \mathrm{CDCl}_{3}\right) \delta 7.54-7.33(\mathrm{~m}, 4 \mathrm{H}), 7.14$ 
(d, $J=7.9 \mathrm{~Hz}, 4 \mathrm{H}), 2.37$ (s, 6H). ${ }^{13} \mathbf{C}$ NMR $\left(101 \mathrm{MHz}, \mathrm{CDCl}_{3}\right) \delta 139.50,132.40,129.22,118.80$, $81.55,73.46,21.64$. The spectroscopic data matched that previously report. ${ }^{5}$

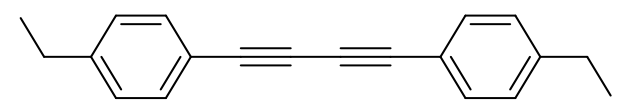

1,4-bis(4-ethylphenyl)buta-1,3-diyne(3e): Following general method. 1-ethyl-4ethynylbenzene (1e; $26.0 \mathrm{mg}, 0.2 \mathrm{mmol}$ ) afforded the title compound as a white solid (23 mg, $89 \%$ ) by using petroleum ether as the column eluent; ${ }^{1} \mathbf{H} \mathbf{N M R}\left(400 \mathrm{MHz}, \mathrm{CDCl}_{3}\right) \delta 7.49-7.40$ (m, 4H), $7.17(\mathrm{~d}, J=8.1 \mathrm{~Hz}, 4 \mathrm{H}), 2.66$ (q, $J=7.6 \mathrm{~Hz}, 4 \mathrm{H}), 1.25(\mathrm{~d}, J=7.6 \mathrm{~Hz}, 6 \mathrm{H}) .{ }^{13} \mathbf{C} \mathbf{~ N M R}$ $\left(101 \mathrm{MHz}, \mathrm{CDCl}_{3}\right) \delta 145.75,132.49,128.03,119.02,81.57,73.45,28.93,15.26$. The spectroscopic data matched that previously report. ${ }^{5}$

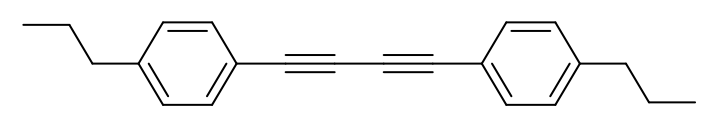

1,4-bis(4-propylphenyl)buta-1,3-diyne(3f): Following general method. 1-ethynyl-4propylbenzene (1f; $28.8 \mathrm{mg}, 0.2 \mathrm{mmol}$ ) afforded the title compound as a yellow solid $(25.2 \mathrm{mg}$, $88 \%$ ) by using petroleum ether as the column eluent; ${ }^{1} \mathbf{H}$ NMR $\left(400 \mathrm{MHz}, \mathrm{CDCl}_{3}\right) \delta 7.44(\mathrm{~d}, J$ $=7.8 \mathrm{~Hz}, 4 \mathrm{H}), 7.14(\mathrm{~d}, J=7.8 \mathrm{~Hz}, 4 \mathrm{H}), 2.59$ (t, $J=7.6 \mathrm{~Hz}, 4 \mathrm{H}), 1.64(\mathrm{q}, J=7.5 \mathrm{~Hz}, 4 \mathrm{H}), 0.94$ (t, $J=7.3 \mathrm{~Hz}, 6 \mathrm{H}) .{ }^{13} \mathbf{C} \mathbf{N M R}\left(101 \mathrm{MHz}, \mathrm{CDCl}_{3}\right) \delta 144.23,132.40,119.04,81.59,73.49,38.06$, $24.28,13.77$. The spectroscopic data matched that previously report. ${ }^{5}$

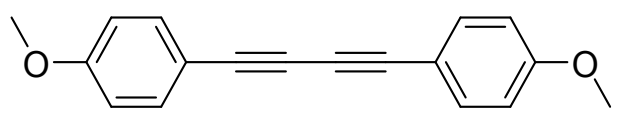

1,4-bis(4-methoxyphenyl)buta-1,3-diyne(3g): Following general method. 1-ethynyl-4methoxybenzene $(\mathbf{1 g} ; 26.4 \mathrm{mg}, 0.2 \mathrm{mmol})$ afforded the title compound as a white solid $(25.2$ mg, 96\%) by using petroleum ether as the column eluent; ${ }^{1} \mathbf{H}$ NMR (400 MHz, $\left.\mathrm{CDCl}_{3}\right) \delta 7.68$ - $7.33(\mathrm{~m}, 4 \mathrm{H}), 7.03$ - $6.75(\mathrm{~m}, 4 \mathrm{H}), 3.82(\mathrm{~s}, 6 \mathrm{H}) .{ }^{13} \mathbf{C} \mathbf{N M R}\left(101 \mathrm{MHz}, \mathrm{CDCl}_{3}\right) \delta 160.24$, $134.04,114.14,113.95,81.23,72.95,55.35$. The spectroscopic data matched that previously report. 5

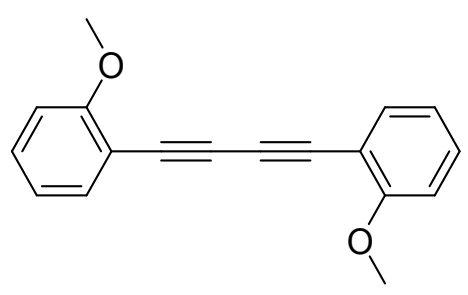


1,4-bis(2-methoxyphenyl)buta-1,3-diyne(3h): Following general method. 1-ethynyl-2methoxybenzene (1h; $26.4 \mathrm{mg}, 0.2 \mathrm{mmol})$ afforded the title compound as a yellow solid (25.2 mg, 96\%) by using petroleum ether as the column eluent; ${ }^{1} \mathbf{H}$ NMR $\left(400 \mathrm{MHz}, \mathrm{CDCl}_{3}\right) \delta 7.48$ (dd, $J=7.6,1.7 \mathrm{~Hz}, 2 \mathrm{H}), 7.32(\mathrm{ddd}, J=8.8,7.6,1.7 \mathrm{~Hz}, 2 \mathrm{H}), 6.99-6.78(\mathrm{~m}, 4 \mathrm{H}), 3.90(\mathrm{~s}, 6 \mathrm{H})$. ${ }^{13}$ C NMR (101 MHz, $\left.\mathrm{CDCl}_{3}\right) \delta 161.34,134.42,130.54,120.51,111.30,110.66,78.65,77.99$, 55.83. The spectroscopic data matched that previously report. ${ }^{8}$

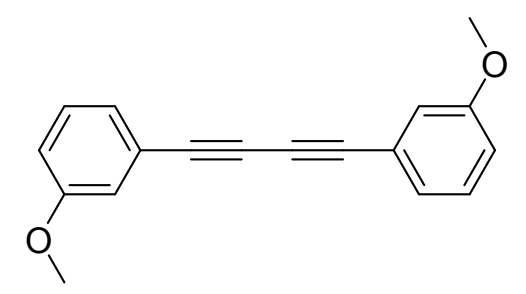

1,4-bis(3-methoxyphenyl)buta-1,3-diyne(3i): Following general method. 1-ethynyl-3methoxybenzene (1i; $26.4 \mathrm{mg}, 0.2 \mathrm{mmol})$ afforded the title compound as a yellow oil $(25.5 \mathrm{mg}$, 97\%) by using petroleum ether as the column eluent; ${ }^{1} \mathbf{H}$ NMR (400 MHz, $\left.\mathrm{CDCl}_{3}\right) \delta 7.26-7.18$ (m, 2H), 7.12 (dt, $J=7.6,1.2 \mathrm{~Hz}, 2 \mathrm{H}), 7.04$ (dd, $J=2.7,1.4 \mathrm{~Hz}, 2 \mathrm{H}), 6.93$ (ddd, $J=8.3,2.6$, $1.0 \mathrm{~Hz}, 2 \mathrm{H}), 3.80(\mathrm{~s}, 6 \mathrm{H}) .{ }^{13} \mathbf{C}$ NMR $\left(101 \mathrm{MHz}, \mathrm{CDCl}_{3}\right) \delta 159.31,129.55,125.09,122.71$, $117.10,116.06,81.52,73.67,55.33$. The spectroscopic data matched that previously report. ${ }^{8}$

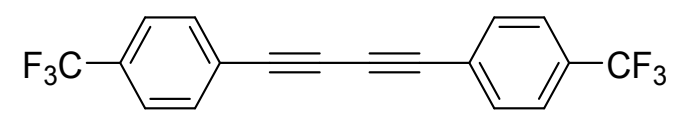

1,4-bis(4-(trifluoromethyl)phenyl)buta-1,3-diyne(3j): Following general method. 1-ethynyl4-(trifluoromethyl)benzene $(\mathbf{1 j} ; 34.0 \mathrm{mg}, 0.2 \mathrm{mmol}$ ) afforded the title compound as a yellow solid (28.8 $\mathrm{mg}, 85 \%)$ by using petroleum ether as the column eluent; ${ }^{1} \mathbf{H}$ NMR (400 MHz, $\left.\mathrm{CDCl}_{3}\right) \delta 7.80-7.49(\mathrm{~m}, 8 \mathrm{H}) .{ }^{13} \mathbf{C} \mathbf{~ N M R}\left(101 \mathrm{MHz}, \mathrm{CDCl}_{3}\right) \delta 132.80,131.58,131.26,130.93$, 130.57, 127.75, 125.50, 125.47, 125.43, 125.39, 125.26, 125.04, 122.33, 119.63, 80.96, 75.63. The spectroscopic data matched that previously report. ${ }^{5}$

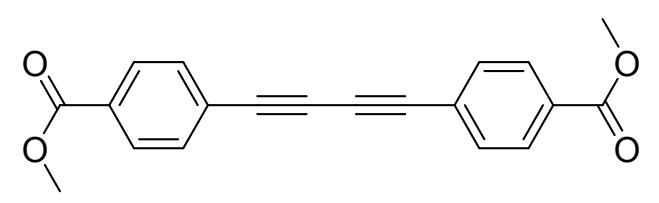

Dimethyl 4,4'-(buta-1,3-diyne-1,4-diyl)dibenzoate(3k): Following general method. Methyl 4-ethynylbenzoate $(\mathbf{1 k} ; 32.0 \mathrm{mg}, 0.2 \mathrm{mmol})$ afforded the title compound as a white solid $(29.6$ mg,93\%) by using petroleum ether : ethyl acetate (10:1) as the column eluent; ${ }^{1} \mathbf{H}$ NMR (400 $\left.\mathrm{MHz} \mathrm{CDCl}_{3}\right) \delta 8.16-7.88(\mathrm{~m}, 4 \mathrm{H}), 7.74-7.46(\mathrm{~m}, 4 \mathrm{H}), 3.93(\mathrm{~s}, 6 \mathrm{H}) .{ }^{13} \mathrm{C}$ NMR $(101 \mathrm{MHz}$, 
$\left.\mathrm{CDCl}_{3}\right) \delta 166.24,132.47,130.55,129.57,126.11,81.85,76.27,52.36$. The spectroscopic data matched that previously report. ${ }^{6}$

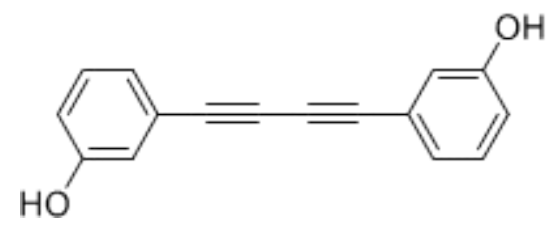

3,3'-(buta-1,3-diyne-1,4-diyl)diphenol (31): Following general method. 3-ethynylphenol (11; $23.6 \mathrm{mg}, 0.2 \mathrm{mmol})$ afforded the title compound as a pale beige solid (21.6 $\mathrm{mg}, 92 \%)$ by using petroleum ether : ethyl acetate (10:1) as the column eluent; ${ }^{1} \mathbf{H}$ NMR (400 MHz, DMSO- $\left.d_{6}\right) \delta$ $9.84(\mathrm{~s}, 2 \mathrm{H}), 7.23$ (t, $J=7.9 \mathrm{~Hz}, 2 \mathrm{H}), 7.02$ (dt, $J=7.6,1.2 \mathrm{~Hz}, 2 \mathrm{H}), 6.93$ (t, $J=2.0 \mathrm{~Hz}, 2 \mathrm{H})$, $6.88(\mathrm{dd}, J=8.2,2.4 \mathrm{~Hz}, 2 \mathrm{H}) .{ }^{13} \mathbf{C}$ NMR $\left(101 \mathrm{MHz}, \mathrm{DMSO}-d_{6}\right) \delta 157.86,130.59,123.72$, $121.68,118.95,118.11,82.25,73.40$. The spectroscopic data matched that previously report. ${ }^{9}$

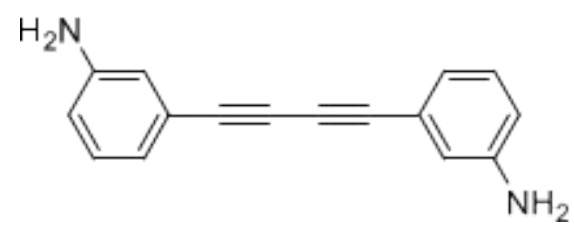

3,3'-(buta-1,3-diyne-1,4-diyl)dianiline(3m): Following general method. 3-ethynylaniline (1 m; $23.4 \mathrm{mg}, 0.2 \mathrm{mmol})$ afforded the title compound as a pale yellow solid (22.8 $\mathrm{mg}, 98 \%$ ) by using petroleum ether :ethyl acetate(5:1) as the column eluent; ${ }^{1} \mathbf{H}$ NMR $\left(400 \mathrm{MHz}, \mathrm{CDCl}_{3}\right) \delta 7.11(\mathrm{t}$, $J=7.8 \mathrm{~Hz}, 2 \mathrm{H}), 6.93(\mathrm{~d}, J=7.6 \mathrm{~Hz}, 2 \mathrm{H}), 6.82(\mathrm{t}, J=1.9 \mathrm{~Hz}, 2 \mathrm{H}), 6.68(\mathrm{dd}, J=8.1,2.4 \mathrm{~Hz}$, 2H), $3.72(\mathrm{~s}, 4 \mathrm{H}) .{ }^{13} \mathrm{C}$ NMR $\left(101 \mathrm{MHz}, \mathrm{CDCl}_{3}\right) \delta 146.29,129.37,122.97,122.49,118.40$, $116.24,81.63,73.38$. The spectroscopic data matched that previously report. ${ }^{5}$

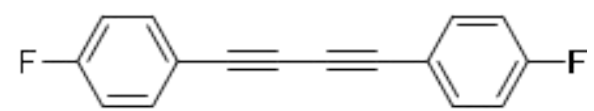

1,4-bis(4-fluorophenyl)buta-1,3-diyne (3n): Following general method. 1-ethynyl-4fluorobenzene (1n; $24.0 \mathrm{mg}, 0.2 \mathrm{mmol}$ ) afforded the title compound as a white solid (22.4 mg,94 \%) by using petroleum ether as the column eluent; ${ }^{1} \mathbf{H}$ NMR $\left(400 \mathrm{MHz}, \mathrm{CDCl}_{3}\right) \delta 7.64$ - 7.39 (m, 4H), 7.22 - 6.90 (m, 4H). ${ }^{13} \mathbf{C}$ NMR (101 MHz, $\left.\mathrm{CDCl}_{3}\right) \delta 164.31,161.81,134.58$, $134.50,117.84,117.80,116.03,115.80,80.43,73.54$. The spectroscopic data matched that previously report. ${ }^{5}$ 


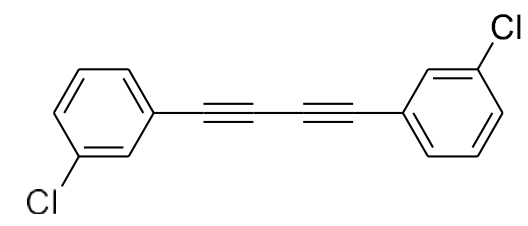

1,4-bis(3-chlorophenyl)buta-1,3-diyne(3o): Following general method. 1-chloro-3ethynylbenzene (1o; $27.3 \mathrm{mg}, 0.2 \mathrm{mmol})$ afforded the title compound as a pale yellow crystals (22.8 mg, 84\%) by using petroleum ether as the column eluent; ${ }^{1} \mathbf{H}$ NMR $\left(400 \mathrm{MHz}, \mathrm{CDCl}_{3}\right) \delta$ $7.51(\mathrm{t}, J=1.8 \mathrm{~Hz}, 2 \mathrm{H}), 7.39(\mathrm{ddt}, J=19.8,8.3,1.4 \mathrm{~Hz}, 4 \mathrm{H}), 7.29(\mathrm{~d}, J=7.8 \mathrm{~Hz}, 2 \mathrm{H}) .{ }^{13} \mathbf{C}$ NMR $\left(101 \mathrm{MHz}, \mathrm{CDCl}_{3}\right) \delta 134.37,132.28,130.67,129.73,123.29,80.56,74.69$. The spectroscopic data matched that previously report. ${ }^{9}$

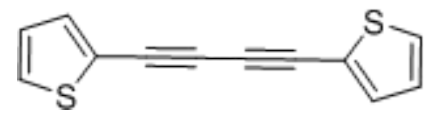

1,4-di(thiophen-2-yl)buta-1,3-diyne (3p): Following general method. 2-ethynylthiophene (1p; $21.6 \mathrm{mg}, 0.2 \mathrm{mmol}$ ) afforded the title compound as a pale brown solid (18.2 $\mathrm{mg}, 85 \%)$ by using petroleum ether : ethyl acetate (30:1) as the column eluent; ${ }^{1} \mathbf{H} \mathbf{~ N M R}\left(400 \mathrm{MHz}, \mathrm{CDCl}_{3}\right) \delta 7.34$ $(\mathrm{dd}, J=8.5,4.4 \mathrm{~Hz}, 4 \mathrm{H}), 7.00(\mathrm{dd}, J=5.1,3.7 \mathrm{~Hz}, 2 \mathrm{H}) .{ }^{13} \mathbf{C} \mathbf{N M R}\left(101 \mathrm{MHz}, \mathrm{CDCl}_{3}\right) \delta 134.42$, $128.93,127.24,121.93,77.79,76.66$. The spectroscopic data matched that previously report. ${ }^{6}$

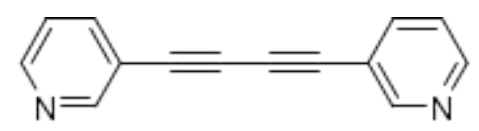

1,4-di(pyridin-3-yl)buta-1,3-diyne (3q): Following general method. 3-ethynylpyridine (1q; $20.6 \mathrm{mg}, 0.2 \mathrm{mmol}$ ) afforded the title compound as a colorless solid (17.0 $\mathrm{mg}, 83 \%)$ by using petroleum ether : ethyl acetate (5:1) as the column eluent; ${ }^{1} \mathbf{H} \mathbf{N M R}\left(600 \mathrm{MHz}, \mathrm{CDCl}_{3}\right) \delta 8.78$ $(\mathrm{s}, 2 \mathrm{H}), 8.61(\mathrm{~s}, 2 \mathrm{H}), 7.81(\mathrm{~d}, J=7.9 \mathrm{~Hz}, 2 \mathrm{H}), 7.49-7.25(\mathrm{~m}, 2 \mathrm{H}) .{ }^{13} \mathbf{C} \mathbf{N M R}\left(151 \mathrm{MHz}, \mathrm{CDCl}_{3}\right)$ $\delta 153.10,149.44,139.39,123.21,118.96,79.20,76.64$. The spectroscopic data matched that previously report. ${ }^{6}$

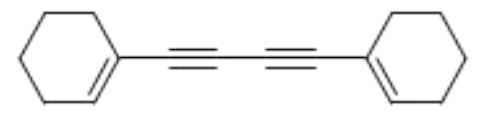

1,4-di(cyclohex-1-en-1-yl)buta-1,3-diyne (3r): Following general method. 1ethynylcyclohex-1-ene (1r; $21.2 \mathrm{mg}, 0.2 \mathrm{mmol})$ afforded the title compound as a white solid (20.2 mg, 96\%) by using petroleum ether as the column eluent; ${ }^{1} \mathbf{H}$ NMR $\left(400 \mathrm{MHz}, \mathrm{CDCl}_{3}\right) \delta$ $6.51-5.90(\mathrm{~m}, 1 \mathrm{H}), 2.11$ (hept, $J=3.0 \mathrm{~Hz}, 4 \mathrm{H}), 1.60$ (dddt, $J=14.2,8.6,6.1,2.6 \mathrm{~Hz}, 4 \mathrm{H}) .{ }^{13} \mathbf{C}$ 
NMR $\left(101 \mathrm{MHz}, \mathrm{CDCl}_{3}\right) \delta 138.11,119.96,82.70,71.56,28.70,25.88,22.14,21.32$. The spectroscopic data matched that previously report. ${ }^{10}$

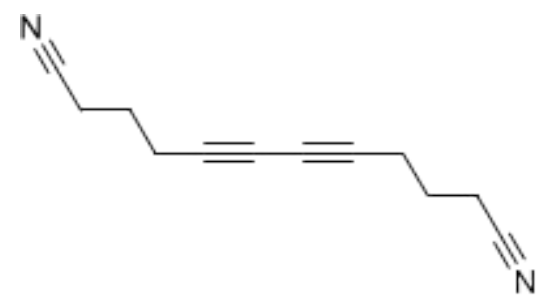

Dodeca-5,7-diynedinitrile (3s): Following general method. Hex-5-ynenitrile (1s; $18.6 \mathrm{mg}, 0.2$ mmol) afforded the title compound as a yellow oil (12.2 $\mathrm{mg}, 66 \%)$ by using petroleum ether : ethyl acetate (20:1) as the column eluent; ${ }^{1} \mathbf{H}$ NMR $\left(400 \mathrm{MHz}, \mathrm{CDCl}_{3}\right) \delta 2.48(\mathrm{dtd}, J=14.1$, 7.0, $1.6 \mathrm{~Hz}, 8 \mathrm{H}), 1.90(\mathrm{pd}, J=6.9,1.6 \mathrm{~Hz}, 4 \mathrm{H}) .{ }^{13} \mathbf{C ~ N M R}\left(151 \mathrm{MHz}, \mathrm{CDCl}_{3}\right) \delta 118.79,75.17$, $66.78,24.23,18.33,16.21$. The spectroscopic data matched that previously report. ${ }^{11}$

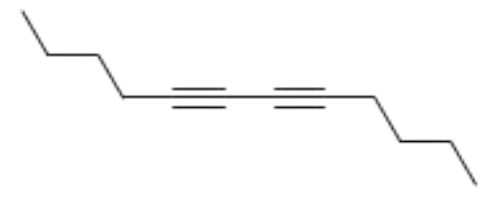

Dodeca-5,7-diyne(3t): Following general method. Hex-1-yne (1t; $16.4 \mathrm{mg}, 0.2 \mathrm{mmol})$ afforded the title compound as a yellow liquid $(9.7 \mathrm{mg}, 60 \%)$ by using petroleum ether as the column eluent; ${ }^{1}$ H NMR (400 MHz, $\left.\mathrm{CDCl}_{3}\right) \delta 2.25(\mathrm{t}, J=6.9 \mathrm{~Hz}, 1 \mathrm{H}), 1.53-1.45(\mathrm{~m}, 1 \mathrm{H}), 1.45-1.37$ $(\mathrm{m}, 1 \mathrm{H}), 1.37-1.15(\mathrm{~m}, 2 \mathrm{H}), 0.90(\mathrm{t}, J=7.2 \mathrm{~Hz}, 2 \mathrm{H}) .{ }^{13} \mathrm{C} \mathrm{NMR}\left(101 \mathrm{MHz}, \mathrm{CDCl}_{3}\right) \delta 77.49$, $65.23,30.39,21.92,18.89,13.54$. The spectroscopic data matched that previously report. ${ }^{5}$

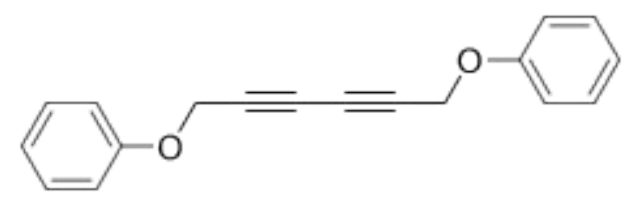

1,6-diphenoxyhexa-2,4-diyne(3u): Following general method. (Prop-2-yn-1-yloxy)benzene $(1 \mathbf{u} ; 26.4 \mathrm{mg}, 0.2 \mathrm{mmol})$ afforded the title compound as a yellow solid (21.5 mg, 82\%) by using petroleum ether as the column eluent; ${ }^{1} \mathbf{H}$ NMR $\left(600 \mathrm{MHz}, \mathrm{CDCl}_{3}\right) \delta 7.39-7.26(\mathrm{~m}, 4 \mathrm{H}), 7.07$ - $6.98(\mathrm{~m}, 2 \mathrm{H}), 6.95(\mathrm{~d}, J=8.2 \mathrm{~Hz}, 4 \mathrm{H}), 4.75(\mathrm{~s}, 4 \mathrm{H}) .{ }^{13} \mathbf{C ~ N M R}\left(151 \mathrm{MHz}, \mathrm{CDCl}_{3}\right) \delta 157.40$, $129.57,121.78,114.88,74.67,71.04,56.18$. The spectroscopic data matched that previously report. $^{12}$

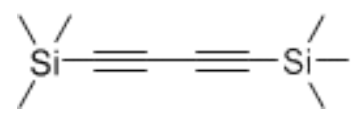


1,4-bis(trimethylsilyl)buta-1,3-diyne(3v): Following general method. Ethynyltrimethylsilane (1v; $19.6 \mathrm{mg}, 0.2 \mathrm{mmol}$ ) afforded the title compound as a white solid (13.6 $\mathrm{mg}, 70 \%$ ) by using petroleum ether as the column eluent; ${ }^{1} \mathbf{H}$ NMR $\left(400 \mathrm{MHz}, \mathrm{CDCl}_{3}\right) \delta 0.07(\mathrm{~s}, 18 \mathrm{H}) .{ }^{13} \mathbf{C ~ N M R}$ $\left(151 \mathrm{MHz}, \mathrm{CDCl}_{3}\right) \delta 87.37,84.04,1.03$. The spectroscopic data matched that previously report. $^{13}$

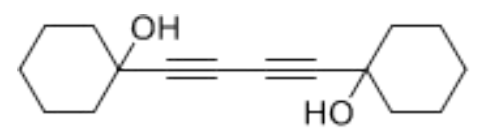

1,1'-(buta-1,3-diyne-1,4-diyl)bis(cyclohexan-1-ol) (3w): Following general method. 1ethynylcyclohexan-1-ol (1w; $24.8 \mathrm{mg}, 0.2 \mathrm{mmol})$ afforded the title compound as a light yellow solid. (20.7 mg, 84\%) by using petroleum ether: ethyl acetate (20:1) as the column eluent; ${ }^{1} \mathbf{H}$ NMR $\left(600 \mathrm{MHz}, \mathrm{CDCl}_{3}\right) \delta 2.19-1.83(\mathrm{~m}, 6 \mathrm{H}), 1.74-1.53(\mathrm{~m}, 14 \mathrm{H}) .{ }^{13} \mathbf{C}$ NMR $(151 \mathrm{MHz}$, $\left.\mathrm{CDCl}_{3}\right) \delta 83.01,69.22,68.34,39.67,25.01,23.11$. The spectroscopic data matched that previously report. ${ }^{11}$<smiles>CC(C)(O)C#CC#CC(C)(C)O</smiles>

2,7-dimethylocta-3,5-diyne-2,7-diol (3x): Following general method. 2-methylbut-3-yn-2-ol (1x; $16.8 \mathrm{mg}, 0.2 \mathrm{mmol}$ ) afforded the title compound as a white solid (11.5 $\mathrm{mg}, 69 \%$ ) by using petroleum ether: ethyl acetate (20:1) as the column eluent; ${ }^{1} \mathbf{H} \mathbf{~ N M R}\left(400 \mathrm{MHz}, \mathrm{CDCl}_{3}\right) \delta 1.53$ $(\mathrm{s}, 12 \mathrm{H}), 1.26(\mathrm{~s}, 2 \mathrm{H}) .{ }^{13} \mathrm{C}$ NMR $\left(101 \mathrm{MHz}, \mathrm{CDCl}_{3}\right) \delta 83.99,66.33,65.58,31.05$. The spectroscopic data matched that previously report. ${ }^{5}$

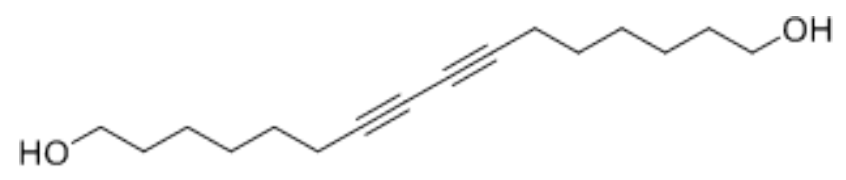

Hexadeca-7,9-diyne-1,16-diol (3y): Following general method. Oct-7-yn-1-ol (1y; 25.2 mg, $0.2 \mathrm{mmol})$ afforded the title compound as a green oil $(18.0 \mathrm{mg}, 72 \%)$ by using petroleum ether: ethyl acetate (5:1) as the column eluent; ${ }^{1} \mathbf{H}$ NMR $\left(400 \mathrm{MHz}, \mathrm{CDCl}_{3}\right) \delta 3.63$ (t, $\left.J=6.6 \mathrm{~Hz}, 4 \mathrm{H}\right)$, $2.26(\mathrm{t}, J=6.9 \mathrm{~Hz}, 4 \mathrm{H}), 1.90(\mathrm{~s}, 2 \mathrm{H}), 1.66-1.48(\mathrm{~m}, 8 \mathrm{H}), 1.47-1.31(\mathrm{~m}, 8 \mathrm{H}) .{ }^{13} \mathrm{C}$ NMR $(101$ $\left.\mathrm{MHz}, \mathrm{CDCl}_{3}\right) \delta 77.41,65.36,62.75,32.53,28.56,28.23,25.22,19.12$. The spectroscopic data matched that previously report. ${ }^{14}$ 


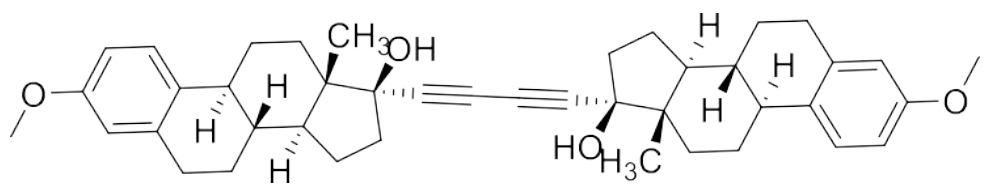

(8R,8'R,9S,9'S,13S,13'S,14S,14'S,17S,17'S)-17,17'-(buta-1,3-diyne-1,4-diyl)bis(3methoxy-13-methyl-7,8,9,11,12,13,14,15,16,17-decahydro-6H-cyclopenta[a]phenanthren17-ol) (3z): Following general method. (8R,9S,13S,14S,17R)-17-ethynyl-3-methoxy-13methyl-7,8,9,11,12,13,14,15,16,17-decahydro-6H-cyclopenta[a]phenanthren-17-ol (1z; 62.1 $\mathrm{mg}, 0.2 \mathrm{mmol}$ ) afforded the title compound as a white solid (34.7 $\mathrm{mg}, 56 \%$ ) by using petroleum ether: ethyl acetate (10:1) as the column eluent; ${ }^{1} \mathbf{H}$ NMR $\left(600 \mathrm{MHz}, \mathrm{CDCl}_{3}\right) \delta 7.21(\mathrm{dd}, J=$ 8.6, $1.1 \mathrm{~Hz}, 2 \mathrm{H}), 6.71(\mathrm{dd}, J=8.6,2.8 \mathrm{~Hz}, 2 \mathrm{H}), 6.63(\mathrm{~d}, J=2.8 \mathrm{~Hz}, 2 \mathrm{H}), 3.78(\mathrm{~s}, 6 \mathrm{H}), 2.86(\mathrm{td}$, $J=7.6,7.1,4.0 \mathrm{~Hz}, 4 \mathrm{H}), 2.43-2.32(\mathrm{~m}, 4 \mathrm{H}), 2.26(\mathrm{td}, J=11.2,4.3 \mathrm{~Hz}, 2 \mathrm{H}), 2.03(\mathrm{ddd}, J=$ 13.7, 11.9, $3.9 \mathrm{~Hz}, 4 \mathrm{H}), 1.87(\mathrm{ddd}, J=12.7,7.2,5.1 \mathrm{~Hz}, 4 \mathrm{H}), 1.82-1.73(\mathrm{~m}, 4 \mathrm{H}), 1.71-1.67$ $(\mathrm{m}, 2 \mathrm{H}), 1.51-1.36(\mathrm{~m}, 8 \mathrm{H}), 0.88(\mathrm{~s}, 6 \mathrm{H}) .{ }^{13} \mathbf{C}$ NMR (151 MHz, $\left.\mathrm{CDCl}_{3}\right) \delta 157.45,137.92$, 132.43, 126.37, 113.81, 111.51, 83.35, 80.69, 70.34, 55.22, 49.86, 47.94, 43.41, 39.45, 38.99, 33.07, 29.82, 27.24, 26.39, 22.96, 12.78. HRMS (ESI) Calcd. for $\mathrm{C}_{42} \mathrm{H}_{50} \mathrm{O}_{4}[\mathrm{M}+\mathrm{H}]^{+}:$: 619.3709, Found: 619.3718 .

\section{b) The list of oxidative cross-coupling reaction}

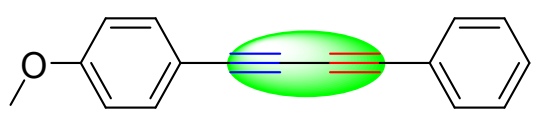

1-methoxy-4-(phenylbuta-1,3-diyn-1-yl)benzene(4a): Following general method. 1-ethynyl4-methoxybenzene (1g, $17.2 \mathrm{mg}, 0.13 \mathrm{mmol})$ and ethynylbenzene (1a, $26.6 \mathrm{mg}, 0.26 \mathrm{mmol})$ afforded the title compound as a colorless solid (24.2 $\mathrm{mg}, 80 \%)$ by using petroleum ether as the column eluent; ${ }^{1} \mathbf{H}$ NMR $\left(400 \mathrm{MHz}, \mathrm{CDCl}_{3}\right) \delta 7.52(\mathrm{~d}, J=7.2 \mathrm{~Hz}, 2 \mathrm{H}), 7.47(\mathrm{t}, J=7.7 \mathrm{~Hz}, 2 \mathrm{H})$, $7.34(\mathrm{q}, J=7.0 \mathrm{~Hz}, 3 \mathrm{H}), 6.86(\mathrm{~d}, J=8.1 \mathrm{~Hz}, 2 \mathrm{H}), 3.82(\mathrm{~d}, J=6.4 \mathrm{~Hz}, 3 \mathrm{H}) .{ }^{13} \mathbf{C}$ NMR $(151$ $\left.\mathrm{MHz}, \mathrm{CDCl}_{3}\right) \delta 160.38,134.14,132.44,129.03,128.42,122.03,114.18,113.72,81.83,81.04$, $74.18,72.75,55.37$. The spectroscopic data matched that previously report. ${ }^{5}$

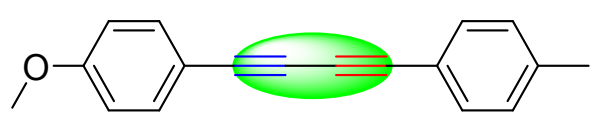

1-methoxy-4-(p-tolylbuta-1,3-diyn-1-yl)benzene(4b): Following general method. 1-ethynyl4-methoxybenzene (1g, $17.2 \mathrm{mg}, 0.13 \mathrm{mmol})$ and 1-ethynyl-4-methylbenzene (1d, $30.2 \mathrm{mg}$, $0.26 \mathrm{mmol})$ afforded the title compound as a white solid $(26.3 \mathrm{mg}, 82 \%)$ by using petroleum ether as the column eluent; ${ }^{1} \mathbf{H}$ NMR $\left(600 \mathrm{MHz}, \mathrm{CDCl}_{3}\right) \delta 7.52-7.44(\mathrm{~m}, 2 \mathrm{H}), 7.41(\mathrm{~d}, J=7.9$ $\mathrm{Hz}, 2 \mathrm{H}), 7.14$ (d, J=7.9 Hz, 2H), $6.97-6.75$ (m, 2H), 3.82 (s, 3H), 2.37 (s, 3H). ${ }^{13} \mathbf{C}$ NMR 
$\left(151 \mathrm{MHz}, \mathrm{CDCl}_{3}\right) \delta 160.30,139.41,134.09,132.36,129.21,118.89,114.15,113.86,81.48$, $73.55,72.88,55.36,21.63$. The spectroscopic data matched that previously report. ${ }^{5}$

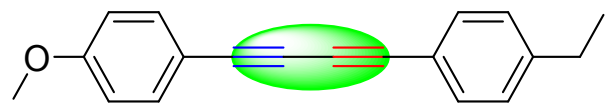

1-ethyl-4-((4-methoxyphenyl)buta-1,3-diyn-1-yl)benzene(4c): Following general method. 1ethynyl-4-methoxybenzene $(\mathbf{1 g}, 17.2 \mathrm{mg}, 0.13 \mathrm{mmol})$ and 1-ethyl-4-ethynylbenzene $(\mathbf{1 e}, 33.9$ $\mathrm{mg}, 0.26 \mathrm{mmol})$ afforded the title compound as a white solid $(26.4 \mathrm{mg}, 78 \%)$ by using petroleum ether as the column eluent; ${ }^{1} \mathbf{H}$ NMR $\left(600 \mathrm{MHz}, \mathrm{CDCl}_{3}\right) \delta 7.65-7.28(\mathrm{~m}, 4 \mathrm{H}), 7.17(\mathrm{~d}, J=8.0$ $\mathrm{Hz}, 2 \mathrm{H}), 6.98-6.73(\mathrm{~m}, 2 \mathrm{H}), 3.82(\mathrm{~s}, 3 \mathrm{H}), 2.66$ (q, $J=7.6 \mathrm{~Hz}, 2 \mathrm{H}), 1.24(\mathrm{t}, J=7.6 \mathrm{~Hz}, 3 \mathrm{H})$. ${ }^{13}$ C NMR $\left(151 \mathrm{MHz}, \mathrm{CDCl}_{3}\right) \delta 160.32,145.69,134.10,132.48,128.04,119.12,114.17,113.87$, $81.49,73.56,55.36,28.95,15.29$. The spectroscopic data matched that previously report. ${ }^{5}$

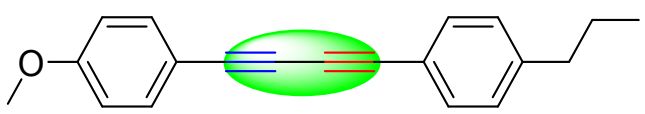

1-methoxy-4-((4-propylphenyl)buta-1,3-diyn-1-yl)benzene(4d): Following general method. 1-ethynyl-4-methoxybenzene (1g; $17.2 \mathrm{mg}, 0.13 \mathrm{mmol})$ and 1-ethynyl-4-propylbenzene (1f, $37.5 \mathrm{mg}, 0.26 \mathrm{mmol})$ afforded the title compound as a yellow solid (28.2 $\mathrm{mg}, 79 \%)$ by using petroleum ether as the column eluent; ${ }^{1} \mathbf{H}$ NMR $\left(600 \mathrm{MHz}, \mathrm{CDCl}_{3}\right) \delta 7.60-7.35(\mathrm{~m}, 4 \mathrm{H}), 7.14$ $(\mathrm{d}, J=8.1 \mathrm{~Hz}, 2 \mathrm{H}), 6.86(\mathrm{~d}, J=8.8 \mathrm{~Hz}, 2 \mathrm{H}), 3.82(\mathrm{~s}, 3 \mathrm{H}), 2.59$ (t, $J=7.6 \mathrm{~Hz}, 2 \mathrm{H}), 1.63$ (q, $J=$ $7.5 \mathrm{~Hz}, 2 \mathrm{H}), 0.94$ (t, $J=7.3 \mathrm{~Hz}, 3 \mathrm{H}) .{ }^{13} \mathbf{C} \mathbf{~ N M R}\left(151 \mathrm{MHz}, \mathrm{CDCl}_{3}\right) \delta 160.29,144.16,134.09$, $132.37,128.61,119.12,114.15,113.87,81.45,81.38,73.54,72.90,55.36,38.05,24.28,13.77$. The spectroscopic data matched that previously report. ${ }^{5}$

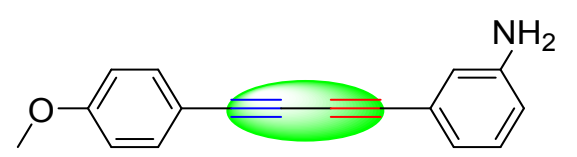

3-((4-methoxyphenyl)buta-1,3-diyn-1-yl)aniline(4e): Following general method. 1-ethynyl4-methoxybenzene (1g; $17.2 \mathrm{mg}, 0.13 \mathrm{mmol})$ and 3-ethynylaniline (1 $\mathbf{m}, 30.5 \mathrm{mg}, 0.26 \mathrm{mmol})$ afforded the title compound as a yellow solid (18.7 $\mathrm{mg}, 58 \%)$ by using petroleum ether : ethyl acetate (5:1) as the column eluent; ${ }^{1} \mathbf{H}$ NMR $\left(600 \mathrm{MHz}, \mathrm{CDCl}_{3}\right) \delta 7.52-7.40(\mathrm{~m}, 2 \mathrm{H}), 7.11(\mathrm{t}$, $J=7.8 \mathrm{~Hz}, 1 \mathrm{H}), 6.92(\mathrm{~d}, J=7.6 \mathrm{~Hz}, 1 \mathrm{H}), 6.91-6.74(\mathrm{~m}, 3 \mathrm{H}), 6.67(\mathrm{dd}, J=8.1,2.3 \mathrm{~Hz}, 1 \mathrm{H})$, $3.82(\mathrm{~s}, 3 \mathrm{H}), 3.79-3.57(\mathrm{~m}, 2 \mathrm{H}) .{ }^{13} \mathbf{C}$ NMR $\left(151 \mathrm{MHz}, \mathrm{CDCl}_{3}\right) \delta 160.32,146.27,134.11$, $129.35,122.94,122.64,118.37,116.14,114.15,113.80,81.53,73.52,72.84,55.36$. The spectroscopic data matched that previously report. ${ }^{5}$ 


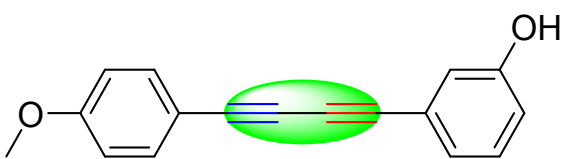

3-((4-methoxyphenyl)buta-1,3-diyn-1-yl)phenol (4f): Following general method. 1-ethynyl4-methoxybenzene (1g; $17.2 \mathrm{mg}, 0.13 \mathrm{mmol}$ ) and 3-ethynylphenol (11, $30.7 \mathrm{mg}, 0.26 \mathrm{mmol})$ afforded the title compound as a yellow solid (24.5 $\mathrm{mg}, 76 \%)$ by using petroleum ether : ethyl acetate (10:1) as the column eluent; ${ }^{1} \mathbf{H}$ NMR (600 MHz, $\left.\mathrm{CDCl}_{3}\right) \delta 7.56-7.43(\mathrm{~m}, 2 \mathrm{H}), 7.20$ $(\mathrm{t}, J=7.9 \mathrm{~Hz}, 1 \mathrm{H}), 7.10(\mathrm{dt}, J=7.7,1.2 \mathrm{~Hz}, 1 \mathrm{H}), 6.98(\mathrm{dd}, J=2.6,1.4 \mathrm{~Hz}, 1 \mathrm{H}), 6.93-6.78(\mathrm{~m}$, 3H), 5.07 (s, 1H), 3.83 (s, 3H). ${ }^{13}$ C NMR $\left(151 \mathrm{MHz}, \mathrm{CDCl}_{3}\right) \delta 160.40,155.31,134.17,129.79$, 125.24, 123.20, 118.88, 116.69, 114.20, 113.65, 81.98, 80.61, 74.21, 72.70, 55.39. HRMS (ESI) Calcd. For $\mathrm{C}_{17} \mathrm{H}_{13} \mathrm{O}_{2}[\mathrm{M}+\mathrm{H}]^{+}:$249.0910, Found: 249.0912.

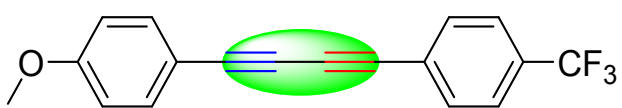

1-methoxy-4-((4-(trifluoromethyl)phenyl)buta-1,3-diyn-1-yl)benzene(4g): $\quad$ Following general method. 1-ethynyl-4-methoxybenzene (1g; $17.2 \mathrm{mg}, 0.13 \mathrm{mmol})$ and 1-ethynyl-4(trifluoromethyl)benzene $(\mathbf{1 j}, 44.2 \mathrm{mg}, 0.26 \mathrm{mmol}$ ) afforded the title compound as a yellow solid (26.5 mg, 68\%) by using petroleum ether as the column eluent; ${ }^{1} \mathbf{H}$ NMR $(600 \mathrm{MHz}$, $\left.\mathrm{CDCl}_{3}\right) \delta 7.69-7.55(\mathrm{~m}, 4 \mathrm{H}), 7.55-7.42(\mathrm{~m}, 2 \mathrm{H}), 6.95-6.80(\mathrm{~m}, 2 \mathrm{H}), 3.83(\mathrm{~s}, 3 \mathrm{H}) .{ }^{13} \mathbf{C}$ NMR $\left(151 \mathrm{MHz}, \mathrm{CDCl}_{3}\right) \delta 160.65,134.27,132.62,130.65,130.43,126.50,125.96,125.34,124.70$, $122.89,114.25,113.25,83.22,79.34,76.58,72.34,55.38$. The spectroscopic data matched that previously report. ${ }^{5}$

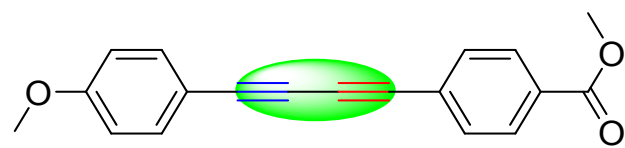

Methyl 4-((4-methoxyphenyl)buta-1,3-diyn-1-yl)benzoate(4h): Following general method. 1-ethynyl-4-methoxybenzene (1g; $17.2 \mathrm{mg}, 0.13 \mathrm{mmol})$ and methyl 4-ethynylbenzoate (1k, 41.6 $\mathrm{mg}, 0.26 \mathrm{mmol})$ afforded the title compound as a yellow solid (21.14 $\mathrm{mg}, 56 \%)$ by using petroleum ether as the column eluent; ${ }^{1} \mathbf{H}$ NMR $\left(600 \mathrm{MHz}, \mathrm{CDCl}_{3}\right) \delta 8.08-7.93(\mathrm{~m}, 2 \mathrm{H}), 7.56$ $(\mathrm{d}, J=8.4 \mathrm{~Hz}, 2 \mathrm{H}), 7.48(\mathrm{~d}, J=8.8 \mathrm{~Hz}, 2 \mathrm{H}), 6.87(\mathrm{~d}, J=8.8 \mathrm{~Hz}, 2 \mathrm{H}), 3.92(\mathrm{~s}, 3 \mathrm{H}), 3.83(\mathrm{~s}, 3 \mathrm{H})$. ${ }^{13}$ C NMR $\left(151 \mathrm{MHz}, \mathrm{CDCl}_{3}\right) \delta 166.37,160.60,134.25,132.30,130.09,129.53,126.76,114.23$, $113.35,83.41,80.03,72.48,55.38,52.32$. The spectroscopic data matched that previously report. $^{15}$

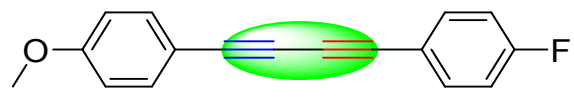


1-fluoro-4-((4-methoxyphenyl)buta-1,3-diyn-1-yl)benzene(4i): Following general method. 1-ethynyl-4-methoxybenzene (1g; $17.2 \mathrm{mg}, 0.13 \mathrm{mmol})$ and 1-ethynyl-4-fluorobenzene (1n, $31.2 \mathrm{mg}, 0.26 \mathrm{mmol})$ afforded the title compound as a white solid (25.4 $\mathrm{mg}, 78 \%)$ by using petroleum ether as the column eluent; ${ }^{1} \mathbf{H} \mathbf{N M R}\left(600 \mathrm{MHz}, \mathrm{CDCl}_{3}\right) \delta 7.78-7.29(\mathrm{~m}, 4 \mathrm{H}), 7.03$ $(\mathrm{t}, J=8.7 \mathrm{~Hz}, 2 \mathrm{H}), 6.95-6.73(\mathrm{~m}, 2 \mathrm{H}), 3.83(\mathrm{~s}, 3 \mathrm{H}) .{ }^{13} \mathbf{C ~ N M R}\left(151 \mathrm{MHz}, \mathrm{CDCl}_{3}\right) \delta 163.75$, $162.09,160.42$, 134.46, 134.41, 134.15, 118.16, 118.13, 115.92, 115.78, 114.19, 113.60, 81.82, $79.92,73.97,72.59,55.36$. The spectroscopic data matched that previously report. ${ }^{5}$

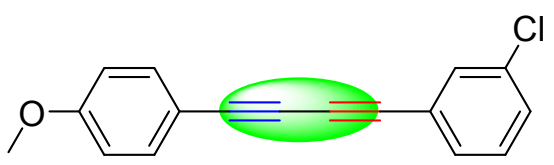

1-chloro-3-((4-methoxyphenyl)buta-1,3-diyn-1-yl)benzene(4j): Following general method. 1-ethynyl-4-methoxybenzene (1g; $17.2 \mathrm{mg}, 0.13 \mathrm{mmol})$ and 1-chloro-3-ethynylbenzene (10, $35.5 \mathrm{mg}, 0.26 \mathrm{mmol})$ afforded the title compound as a yellow solid (24.3 $\mathrm{mg}, 70 \%)$ by using petroleum ether as the column eluent; ${ }^{1} \mathbf{H}$ NMR $\left(600 \mathrm{MHz}, \mathrm{CDCl}_{3}\right) \delta 7.55-7.43(\mathrm{~m}, 3 \mathrm{H}), 7.38$ (dt, $J=7.7,1.4 \mathrm{~Hz}, 1 \mathrm{H}), 7.33$ (ddd, $J=8.1,2.1,1.1 \mathrm{~Hz}, 1 \mathrm{H}), 7.25$ (t, $J=7.9 \mathrm{~Hz}, 1 \mathrm{H}), 6.93$ $6.82(\mathrm{~m}, 2 \mathrm{H}), 3.82$ (s, 3H). ${ }^{13} \mathbf{C}$ NMR (151 MHz, $\left.\mathrm{CDCl}_{3}\right) \delta 160.54,134.28,134.23,132.14$, $130.55,129.66,129.32,123.82,114.22,113.40,82.63,79.39,75.41,72.46,55.38$. The spectroscopic data matched that previously report. ${ }^{\mathbf{1 6}}$

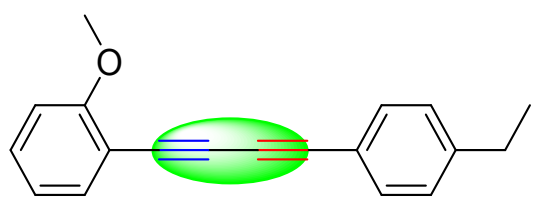

1-((4-ethylphenyl)buta-1,3-diyn-1-yl)-2-methoxybenzene(4k): Following general method. 1-ethynyl-2-methoxybenzene $(\mathbf{1 h} ; 17.18 \mathrm{mg}, 0.13 \mathrm{mmol})$ and 1-ethyl-4-ethynylbenzene (1e, $33.9 \mathrm{mg}, 0.26 \mathrm{mmol})$ afforded the title compound as a yellow oily liquid (24.4 $\mathrm{mg}, 72 \%$ ) by using petroleum ether as the column eluent; ${ }^{1} \mathbf{H}$ NMR $\left(600 \mathrm{MHz}, \mathrm{CDCl}_{3}\right) \delta 7.63-7.32(\mathrm{~m}, 4 \mathrm{H})$, $7.17(\mathrm{~d}, J=7.9 \mathrm{~Hz}, 2 \mathrm{H}), 6.99-6.75(\mathrm{~m}, 2 \mathrm{H}), 3.82(\mathrm{~s}, 3 \mathrm{H}), 2.66(\mathrm{q}, J=7.6 \mathrm{~Hz}, 2 \mathrm{H}), 1.24(\mathrm{t}, J$ $=7.6 \mathrm{~Hz}, 3 \mathrm{H}) .{ }^{13} \mathbf{C}$ NMR $\left(151 \mathrm{MHz}, \mathrm{CDCl}_{3}\right) \delta 160.32,145.69,134.10,132.48,128.04,119.12$, $114.17,113.87,81.49,81.39,73.56,72.92,55.36,28.95,15.29$. HRMS (ESI) Calcd. for $\mathrm{C}_{19} \mathrm{H}_{16} \mathrm{O}[\mathrm{M}+\mathrm{H}]^{+}:$261.1201, Found: 261.1213.

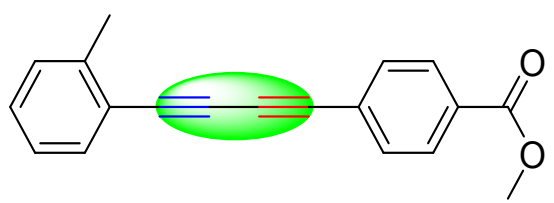


Methyl 4-(o-tolylbuta-1,3-diyn-1-yl)benzoate(4l): Following general method. 1-ethynyl-2methylbenzene (1b; $15.1 \mathrm{mg}, 0.13 \mathrm{mmol})$ and methyl 4-ethynylbenzoate (1k, $41.6 \mathrm{mg}, 0.26$ $\mathrm{mmol}$ ) afforded the title compound as a yellow solid $(24.9 \mathrm{mg}, 70 \%)$ by using petroleum ether as the column eluent; ${ }^{1} \mathbf{H}$ NMR $\left(400 \mathrm{MHz}, \mathrm{CDCl}_{3}\right) \delta 8.14-7.87(\mathrm{~m}, 2 \mathrm{H}), 7.58(\mathrm{~d}, J=8.3 \mathrm{~Hz}$, 2H), $7.50(\mathrm{dd}, J=7.6,1.3 \mathrm{~Hz}, 1 \mathrm{H}), 7.31-7.11(\mathrm{~m}, 3 \mathrm{H}), 3.92(\mathrm{~s}, 3 \mathrm{H}), 2.49$ (s, 3H). ${ }^{13} \mathbf{C} \mathbf{N M R}$ $\left(101 \mathrm{MHz}, \mathrm{CDCl}_{3}\right) \delta 166.34,141.87,133.04,132.35,130.21,129.66,129.55,129.48,126.61$, 125.74, 121.27, 82.14, 81.04, 76.85, 52.35, 20.75. HRMS (ESI) Calcd. for $\mathrm{C}_{19} \mathrm{H}_{14} \mathrm{O}_{2}[\mathrm{M}+\mathrm{H}]^{+}$: 275.0994, Found: 275.0999.

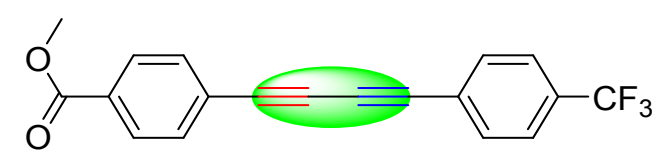

Methyl 4-((4-(trifluoromethyl)phenyl)buta-1,3-diyn-1-yl)benzoate(4m): Following general method. Methyl 4-ethynylbenzoate $(\mathbf{1 k}, 20.8 \mathrm{mg}, 0.13 \mathrm{mmol})$ and 1-ethynyl-4(trifluoromethyl)benzene (1jj, $44.2 \mathrm{mg}, 0.26 \mathrm{mmol}$ ) afforded the title compound as a yellow solid (32.4 mg, 76\%) by using petroleum ether as the column eluent; ${ }^{1} \mathbf{H}$ NMR (600 MHz, $\left.\mathrm{CDCl}_{3}\right) \delta 8.10-7.94(\mathrm{~m}, 2 \mathrm{H}), 7.82-7.43(\mathrm{~m}, 6 \mathrm{H}), 3.93(\mathrm{~s}, 3 \mathrm{H}) .{ }^{13} \mathbf{C ~ N M R}\left(151 \mathrm{MHz}, \mathrm{CDCl}_{3}\right)$ $\delta 166.24,132.79,132.49,131.34,131.13,130.91,130.62,129.59,126.00,125.45,125.43$, 125.40, 124.60, 122.80, 81.68, 81.13, 76.09, 75.80, 52.37. HRMS (ESI) Calcd. for $\mathrm{C}_{19} \mathrm{H}_{12} \mathrm{~F}_{3} \mathrm{O}_{2}$ $[\mathrm{M}+\mathrm{H}]^{+}:$329.0784, Found: 329.0781 .

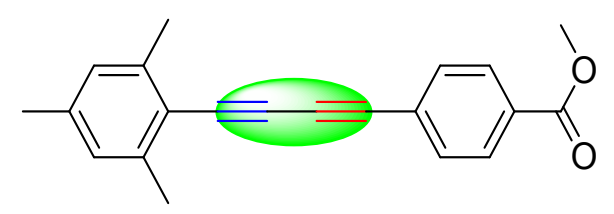

Methyl 4-(mesitylbuta-1,3-diyn-1-yl)benzoate(4n): Following general method. 2-ethynyl1,3,5-trimethylbenzene (1c; $18.8 \mathrm{mg}, 0.13 \mathrm{mmol})$ and methyl 4-ethynylbenzoate $(\mathbf{1 k}, 41.6 \mathrm{mg}$, $0.26 \mathrm{mmol})$ afforded the title compound as a yellow solid $(25.6 \mathrm{mg}, 65 \%)$ by using petroleum ether as the column eluent; ${ }^{1} \mathbf{H}$ NMR $\left(600 \mathrm{MHz}, \mathrm{CDCl}_{3}\right) \delta 8.01(\mathrm{~d}, J=8.4 \mathrm{~Hz}, 2 \mathrm{H}), 7.59(\mathrm{~d}, J$ $=8.4 \mathrm{~Hz}, 2 \mathrm{H}), 6.88(\mathrm{~s}, 2 \mathrm{H}), 3.93(\mathrm{~s}, 3 \mathrm{H}), 2.45(\mathrm{~s}, 6 \mathrm{H}), 2.29(\mathrm{~s}, 3 \mathrm{H}) .{ }^{13} \mathbf{C ~ N M R}\left(151 \mathrm{MHz}, \mathrm{CDCl}_{3}\right)$ $\delta 166.39,142.08,139.23,132.26,130.06,129.53,127.84,126.89,118.29,81.47,81.40,80.47$, 77.17, 52.32, 21.48, 20.97. HRMS (ESI) Calcd. for $\mathrm{C}_{21} \mathrm{H}_{18} \mathrm{O}_{2}[\mathrm{M}+\mathrm{H}]^{+}:$303.1307, Found: 303.1312 . 


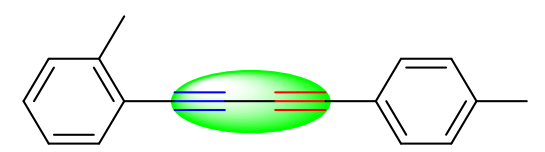

1-methyl-2-(p-tolylbuta-1,3-diyn-1-yl)benzene(4o): Following general method. 1-ethynyl-2methylbenzene (1b; $15.1 \mathrm{mg}, 0.13 \mathrm{mmol})$ and 1-ethynyl-4-methylbenzene (1d, $30.2 \mathrm{mg}, 0.26$ mmol) afforded the title compound as a white solid $(21.6 \mathrm{mg}, 72 \%)$ by using petroleum ether as the column eluent; ${ }^{1} \mathbf{H}$ NMR $\left(400 \mathrm{MHz}, \mathrm{CDCl}_{3}\right) \delta 7.41(\mathrm{dd}, J=7.7,1.4 \mathrm{~Hz}, 1 \mathrm{H}), 7.35(\mathrm{~d}, J$ $=8.2 \mathrm{~Hz}, 2 \mathrm{H}), 7.21-7.12(\mathrm{~m}, 2 \mathrm{H}), 7.12-7.02(\mathrm{~m}, 3 \mathrm{H}), 2.41(\mathrm{~s}, 3 \mathrm{H}), 2.29(\mathrm{~s}, 3 \mathrm{H}) .{ }^{13} \mathbf{C} \mathbf{N M R}$ $\left(101 \mathrm{MHz}, \mathrm{CDCl}_{3}\right) \delta 141.67,139.55,132.93,132.39,129.57,129.24,129.07,125.66,121.75$, $118.79,82.41,80.29,77.62,73.43,21.65,20.75$. The spectroscopic data matched that previously report. ${ }^{17}$

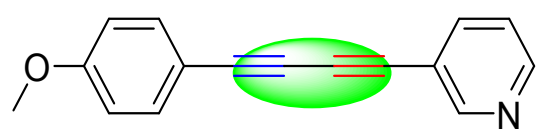

3-((4-methoxyphenyl)buta-1,3-diyn-1-yl)pyridine(4p): Following general method. 1ethynyl-4-methoxybenzene (1g; $17.2 \mathrm{mg}, 0.13 \mathrm{mmol})$ and 3-ethynylpyridine (1q, $26.8 \mathrm{mg}, 0.26$ mmol) afforded the title compound as a brown solid $(18.50 \mathrm{mg}, 61 \%)$ by using petroleum ether : ethyl acetate (5:1) as the column eluent; ${ }^{1} \mathbf{H}$ NMR $\left(600 \mathrm{MHz}, \mathrm{CDCl}_{3}\right) \delta 8.85-8.65(\mathrm{~m}, 1 \mathrm{H})$, $8.56(\mathrm{dd}, J=4.9,1.7 \mathrm{~Hz}, 1 \mathrm{H}), 7.78(\mathrm{dt}, J=7.9,1.9 \mathrm{~Hz}, 1 \mathrm{H}), 7.48(\mathrm{~d}, J=8.8 \mathrm{~Hz}, 2 \mathrm{H}), 7.30-$ $7.25(\mathrm{~m}, 1 \mathrm{H}), 6.95-6.82(\mathrm{~m}, 2 \mathrm{H}), 3.83(\mathrm{~s}, 3 \mathrm{H}) .{ }^{13} \mathbf{C ~ N M R}\left(151 \mathrm{MHz}, \mathrm{CDCl}_{3}\right) \delta 160.63,153.03$, 149.01, 139.23, 134.26, 123.04, 119.51, 114.24, 113.22, 83.12, 77.53, 77.48, 72.28, 55.38. HRMS (ESI) Calcd. for $\mathrm{C}_{16} \mathrm{H}_{11} \mathrm{ON}[\mathrm{M}+\mathrm{H}]^{+}$: 234.0841, Found: 234.0849.

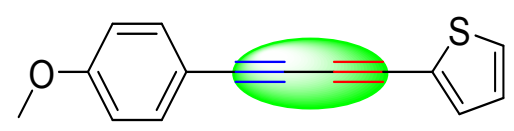

2-((4-methoxyphenyl)buta-1,3-diyn-1-yl)thiophene(4q): Following general method. 1ethynyl-4-methoxybenzene $(\mathbf{1 g} ; 17.2 \mathrm{mg}, 0.13 \mathrm{mmol})$ and 2-ethynylthiophene $(\mathbf{1 p}, 28.1 \mathrm{mg}$, $0.26 \mathrm{mmol}$ ) afforded the title compound as a brown solid $(18.6 \mathrm{mg}, 60 \%)$ by using petroleum ether as the column eluent; ${ }^{1} \mathbf{H}$ NMR $\left(600 \mathrm{MHz}, \mathrm{CDCl}_{3}\right) \delta 7.47(\mathrm{~d}, J=8.8 \mathrm{~Hz}, 2 \mathrm{H}), 7.33(\mathrm{dd}, J$ $=3.7,1.1 \mathrm{~Hz}, 1 \mathrm{H}), 7.30(\mathrm{dd}, J=5.1,1.1 \mathrm{~Hz}, 1 \mathrm{H}), 6.99(\mathrm{dd}, J=5.2,3.7 \mathrm{~Hz}, 1 \mathrm{H}), 6.86(\mathrm{~d}, J=$ $8.8 \mathrm{~Hz}, 2 \mathrm{H}), 3.83$ (s, 3H). ${ }^{13} \mathbf{C}$ NMR (151 MHz, $\left.\mathrm{CDCl}_{3}\right) \delta 160.47,134.14,134.02,128.45$, $127.15,122.33,114.20,113.58,83.92,78.23,74.02,72.59,55.38$. The spectroscopic data matched that previously report. ${ }^{18}$ 


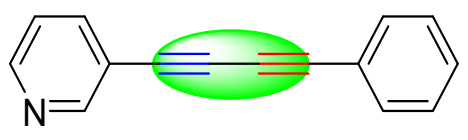

3-(phenylbuta-1,3-diyn-1-yl)pyridine(4r): Following general method. 3-ethynylpyridine (1q, $13.4 \mathrm{mg}, 0.13 \mathrm{mmol}$ ) and phenylacetylene (1a; $26.6 \mathrm{mg}, 0.26 \mathrm{mmol}$ ) afforded the title compound as a brownish solid $(22.7 \mathrm{mg}, 86 \%)$ by using methanol: DCM (1:100) as the column eluent; ${ }^{1} \mathbf{H}$ NMR $\left(600 \mathrm{MHz}, \mathrm{CDCl}_{3}\right) \delta 8.76(\mathrm{~d}, J=2.1 \mathrm{~Hz}, 1 \mathrm{H}), 8.57(\mathrm{dd}, J=4.9,1.7 \mathrm{~Hz}, 1 \mathrm{H})$, $7.79(\mathrm{dt}, J=7.9,1.9 \mathrm{~Hz}, 1 \mathrm{H}), 7.60-7.49(\mathrm{~m}, 2 \mathrm{H}), 7.43-7.31(\mathrm{~m}, 3 \mathrm{H}), 7.27(\mathrm{dd}, J=8.0,4.8$ $\mathrm{Hz}, 1 \mathrm{H}) .{ }^{13} \mathbf{C}$ NMR $\left(151 \mathrm{MHz}, \mathrm{CDCl}_{3}\right) \delta 153.09,149.20,139.30,132.59,129.56,128.52$, $123.06,121.34,119.28,82.80,77.99,77.24,73.41$. The spectroscopic data matched that previously report. ${ }^{19-20}$

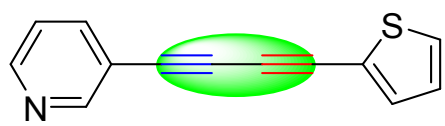

3-(thiophen-2-ylbuta-1,3-diyn-1-yl)pyridine(4s): Following general method. 3ethynylpyridine (1q, $13.4 \mathrm{mg}, 0.13 \mathrm{mmol})$ and 2-ethynylthiophene (1p; $28.1 \mathrm{mg}, 0.26 \mathrm{mmol})$ afforded the title compound as a brown yellow solid (19.3 $\mathrm{mg}, 71 \%)$ by using petroleum ether : ethyl acetate (5:1) as the column eluent; ${ }^{1} \mathbf{H}$ NMR (400 MHz, $\left.\mathrm{CDCl}_{3}\right) \delta 8.69(\mathrm{~s}, 1 \mathrm{H}), 8.51(\mathrm{~d}, J$ $=4.9 \mathrm{~Hz}, 1 \mathrm{H}), 7.72(\mathrm{dt}, J=7.9,1.9 \mathrm{~Hz}, 1 \mathrm{H}), 7.44-7.23(\mathrm{~m}, 2 \mathrm{H}), 7.23-7.16(\mathrm{~m}, 1 \mathrm{H}), 6.94$ $(\mathrm{dd}, J=5.1,3.7 \mathrm{~Hz}, 1 \mathrm{H}) .{ }^{13} \mathrm{C}$ NMR $\left(101 \mathrm{MHz}, \mathrm{CDCl}_{3}\right) \delta 153.03,149.27,139.25,134.74$, 129.19, 127.27, 123.08, 121.57, 119.20, 80.06, 77.44, 75.85. HRMS (ESI) Calcd. for $\mathrm{C}_{13} \mathrm{H}_{7} \mathrm{NS}$ $[\mathrm{M}+\mathrm{H}]^{+}:$210.0299, Found: 210.0311 .

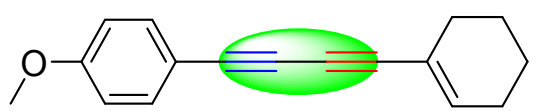

1-(cyclohex-1-en-1-ylbuta-1,3-diyn-1-yl)-4-methoxybenzene(4t): Following general method. 1-ethynyl-4-methoxybenzene (1g; $17.2 \mathrm{mg}, 0.13 \mathrm{mmol})$ and 1-ethynylcyclohex-1-ene (1o, 27.6 $\mathrm{mg}, 0.26 \mathrm{mmol})$ afforded the title compound as a green solid $(22.1 \mathrm{mg}, 72 \%)$ by using petroleum ether : ethyl acetate (20:1) as the column eluent; ${ }^{1} \mathbf{H}$ NMR $\left(600 \mathrm{MHz}, \mathrm{CDCl}_{3}\right) \delta 7.59-7.27(\mathrm{~m}$, $2 \mathrm{H}), 7.05-6.72(\mathrm{~m}, 2 \mathrm{H}), 6.29(\mathrm{t}, J=2.0 \mathrm{~Hz}, 1 \mathrm{H}), 3.81(\mathrm{~s}, 3 \mathrm{H}), 2.14$ (dddd, $J=17.0,7.9,4.2$, $1.9 \mathrm{~Hz}, 4 \mathrm{H}), 1.68-1.61(\mathrm{~m}, 2 \mathrm{H}), 1.61-1.57$ (m, 2H). ${ }^{13} \mathbf{C} \mathbf{N M R}\left(151 \mathrm{MHz}, \mathrm{CDCl}_{3}\right) \delta 160.13$, 138.47, 133.97, 119.90, 114.09, 83.20, 80.72, 72.96, 71.56, 55.33, 28.70, 25.93, 22.15, 21.33. The spectroscopic data matched that previously report. ${ }^{21}$ 


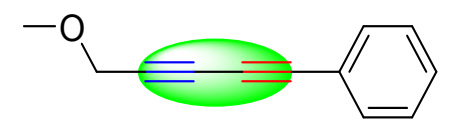

(5-methoxypenta-1,3-diyn-1-yl)benzene(4u): Following general method.3-methoxyprop-1yne (1; $9.1 \mathrm{mg}, 0.13 \mathrm{mmol})$ and ethynylbenzene (1a, $26.6 \mathrm{mg}, 0.26 \mathrm{mmol})$ afforded the title compound as a colorless liquid ( $15.3 \mathrm{mg}, 69 \%)$ by using petroleum ether : ethyl acetate $(50: 1)$ as the column eluent; ${ }^{1} \mathbf{H}$ NMR $\left(400 \mathrm{MHz}, \mathrm{CDCl}_{3}\right) \delta 7.52-7.35(\mathrm{~m}, 2 \mathrm{H}), 7.33-7.18(\mathrm{~m}, 3 \mathrm{H})$, $4.18(\mathrm{~s}, 2 \mathrm{H}), 3.36$ (s, 3H). ${ }^{13} \mathrm{C}$ NMR (101 MHz, $\left.\mathrm{CDCl}_{3}\right) \delta$ 132.61, 129.33, 128.42, 121.44, $78.61,78.07,73.29,71.09,60.38,57.86$. The spectroscopic data matched that previously report. $^{22}$

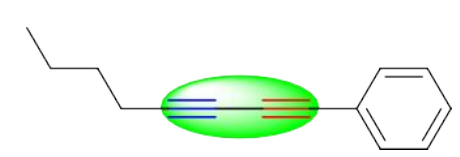

Octa-1,3-diyn-1-ylbenzene (4v): Following general method. Hex-1-yne (1t; 10.7 mg, 0.13 $\mathrm{mmol}$ ) and phenylacetylene (1a, $26.6 \mathrm{mg}, 0.26 \mathrm{mmol})$ afforded the title compound as a colourless liquid (14.2 mg, 60\%) by using petroleum ether as the column eluent; ${ }^{1} \mathbf{H}$ NMR (600 $\left.\mathrm{MHz}, \mathrm{CDCl}_{3}\right) \delta 7.66-7.38(\mathrm{~m}, 2 \mathrm{H}), 7.40-7.26(\mathrm{~m}, 3 \mathrm{H}), 2.37$ (td, $\left.J=7.1,1.9 \mathrm{~Hz}, 2 \mathrm{H}\right), 1.62-$ $1.53(\mathrm{~m}, 2 \mathrm{H}), 1.52-1.35$ (m, 2H), 0.94 (td, $J=7.3,1.9 \mathrm{~Hz}, 3 \mathrm{H}) .{ }^{13} \mathbf{C} \mathbf{~ N M R}\left(151 \mathrm{MHz}, \mathrm{CDCl}_{3}\right)$ $\delta 132.50,128.79,128.34,122.15,84.86,74.69,74.40,65.05,30.30,21.98,19.30,13.56$. The spectroscopic data matched that previously report. ${ }^{22}$

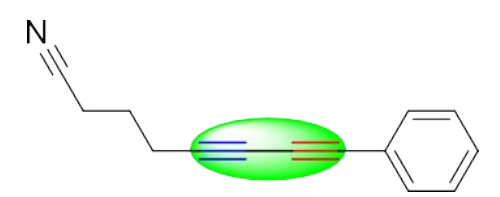

8-phenylocta-5,7-diynenitrile (4w): Following general method. Hex-5-ynenitrile (1s; $12.1 \mathrm{mg}$, $0.13 \mathrm{mmol}$ ) and phenylacetylene (1a, $26.6 \mathrm{mg}, 0.26 \mathrm{mmol})$ afforded the title compound as an colorless liquid (14.6 mg, 58\%) by using petroleum ether: ethyl acetate (10:1) as the column eluent; ${ }^{1} \mathbf{H}$ NMR $\left(600 \mathrm{MHz}, \mathrm{CDCl}_{3}\right) \delta 7.61-7.42(\mathrm{~m}, 2 \mathrm{H}), 7.42-7.26(\mathrm{~m}, 3 \mathrm{H}), 2.55$ (dt, $J=$ 16.6, 7.0 Hz, 4H), 1.94 (p, J=7.0 Hz, 2H). $\left.{ }^{13} \mathbf{C ~ N M R ~ ( 1 5 1 ~ M H z , ~} \mathrm{CDCl}_{3}\right) \delta 132.57,129.16$, $128.42,121.62,118.83,81.12,75.77,73.73,67.03,24.30,18.74,16.25$. The spectroscopic data matched that previously report. ${ }^{22}$

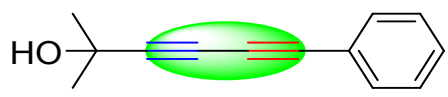

2-methyl-6-phenylhexa-3,5-diyn-2-ol(4x): Following general method.2-methylbut-3-yn-2-ol 
(1x; $10.9 \mathrm{mg}, 0.13 \mathrm{mmol}$ ) and phenylacetylene (1a, $26.6 \mathrm{mg}, 0.26 \mathrm{mmol}$ ) afforded the title compound as an white solid (16.8 $\mathrm{mg}, 70 \%)$ by using petroleum ether: ethyl acetate $(5: 1)$ as the column eluent; ${ }^{1} \mathbf{H}$ NMR $\left(600 \mathrm{MHz}, \mathrm{CDCl}_{3}\right) \delta 7.48-7.37$ (m, 2H), $7.37-7.21(\mathrm{~m}, 3 \mathrm{H}), 2.03$ $(\mathrm{s}, 1 \mathrm{H}), 1.53(\mathrm{~s}, 6 \mathrm{H}) .{ }^{13} \mathrm{C}$ NMR $\left(151 \mathrm{MHz}, \mathrm{CDCl}_{3}\right) \delta$ 132.52, 129.26, 128.43, 121.55, 86.67, $78.82,73.12,67.07,65.77,31.14$. The spectroscopic data matched that previously report. ${ }^{17}$

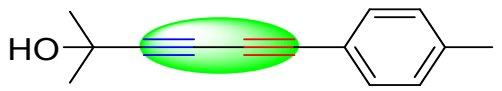

2-methyl-6-(p-tolyl)hexa-3,5-diyn-2-ol(4y): Following general method. 2-methylbut-3-yn-2ol (1x; $10.9 \mathrm{mg}, 0.13 \mathrm{mmol})$ and 1-ethynyl-4-methylbenzene (1d, $30.2 \mathrm{mg}, 0.26 \mathrm{mmol})$ afforded the title compound as an pale yellow solid $(18.0 \mathrm{mg}, 70 \%)$ by using petroleum ether: ethyl acetate (5:1) as the column eluent; ${ }^{1} \mathbf{H}$ NMR $\left(600 \mathrm{MHz}, \mathrm{CDCl}_{3}\right) \delta 7.37(\mathrm{~d}, J=7.9 \mathrm{~Hz}, 2 \mathrm{H}), 7.12$ $(\mathrm{d}, J=7.9 \mathrm{~Hz}, 2 \mathrm{H}), 2.35$ (s, 3H), $2.01(\mathrm{~s}, 1 \mathrm{H}), 1.58(\mathrm{~s}, 6 \mathrm{H}),{ }^{13} \mathbf{C} \mathbf{N M R}\left(151 \mathrm{MHz}, \mathrm{CDCl}_{3}\right) \delta$ $139.66,132.44,129.21,118.41,86.33,79.13,72.50,67.21,65.78,31.16,21.62$. The spectroscopic data matched that previously report. ${ }^{17}$

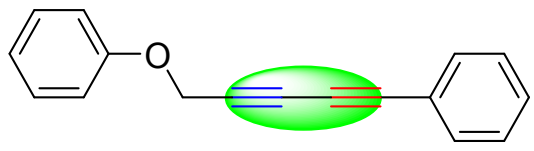

(5-phenoxypenta-1,3-diyn-1-yl)benzene(4z) : Following general method. (Prop-2-yn-1yloxy)benzene (1u; $17.2 \mathrm{mg}, 0.13 \mathrm{mmol})$ and ethynylbenzene (1a, $26.6 \mathrm{mg}, 0.26 \mathrm{mmol})$ afforded the title compound as an green oily liquid $(21.1 \mathrm{mg}, 70 \%)$ by using petroleum ether as the column eluent; ${ }^{1} \mathbf{H}$ NMR $\left(400 \mathrm{MHz}, \mathrm{CDCl}_{3}\right) \delta 7.47-7.34(\mathrm{~m}, 2 \mathrm{H}), 7.33-7.14(\mathrm{~m}, 5 \mathrm{H})$, $7.13-6.71(\mathrm{~m}, 3 \mathrm{H}), 4.76(\mathrm{~s}, 2 \mathrm{H}) .{ }^{13} \mathbf{C}$ NMR $\left(101 \mathrm{MHz}, \mathrm{CDCl}_{3}\right) \delta 157.52,132.65,129.57$, $129.45,128.45,121.71,121.26,114.93,78.79,77.26,73.19,71.82,56.45$. HRMS (ESI) Calcd. for $\mathrm{C}_{17} \mathrm{H}_{12} \mathrm{O}[\mathrm{M}+\mathrm{H}]^{+}:$233.0888, Found: 233.0902 .

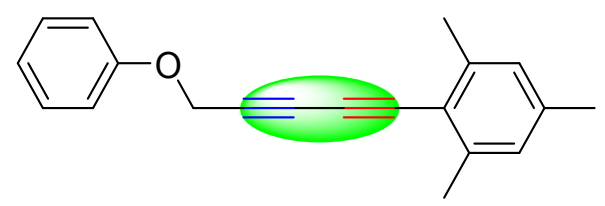

1,3,5-trimethyl-2-(5-phenoxypenta-1,3-diyn-1-yl)benzene(4aa): Following general method. (Prop-2-yn-1-yloxy)benzene (1u; $17.2 \mathrm{mg}, 0.13 \mathrm{mmol}$ ) and 2-ethynyl-1,3,5-trimethylbenzene (1c, $37.5 \mathrm{mg}, 0.26 \mathrm{mmol}$ ) afforded the title compound as an green oily liquid (26.8 $\mathrm{mg}, 75 \%$ ) by using petroleum ether as the column eluent; ${ }^{1} \mathbf{H}$ NMR $(400 \mathrm{MHz}, \mathrm{CDCl} 3) \delta 7.39-7.28(\mathrm{~m}$, 2H), $7.09-6.96$ (m, 3H), 6.85 (s, 2H), 4.86 (s, 2H), 2.39 (s, 6H), 2.28 (s, 3H). ${ }^{13}$ C NMR (151 $\left.\mathrm{MHz}, \mathrm{CDCl}_{3}\right) \delta 157.62,142.14,139.09,129.56,127.76,121.64,118.04,114.89,80.03,77.99$, 
77.09, 72.10, 56.62, 21.44, 20.90. HRMS (ESI) Calcd. for $\mathrm{C}_{20} \mathrm{H}_{18} \mathrm{O}[\mathrm{M}+\mathrm{H}]^{+}:$275.1358, Found: 275.1362 .

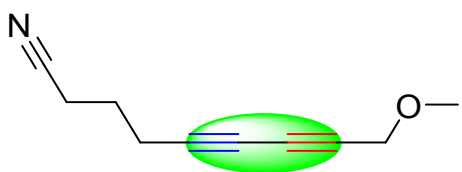

9-methoxynona-5,7-diynenitrile(4ab): Following general method. Hex-5-ynenitrile (1s; 12.1 $\mathrm{mg}, 0.13 \mathrm{mmol}$ ) and 3-methoxyprop-1-yne (1, $18.2 \mathrm{mg}, 0.26 \mathrm{mmol})$ afforded the title compound as an yellow liquid (14.7 $\mathrm{mg}, 70 \%)$ by using petroleum ether : ethyl acetate (5:1)as the column eluent; ${ }^{1}$ H NMR $\left(400 \mathrm{MHz}, \mathrm{CDCl}_{3}\right) \delta 4.15$ (d, $\left.J=1.1 \mathrm{~Hz}, 2 \mathrm{H}\right), 3.38$ (s, 3H), 2.49 (td, $J=7.0$, $5.4 \mathrm{~Hz}, 4 \mathrm{H}), 1.90$ (p, $J=7.0 \mathrm{~Hz}, 2 \mathrm{H}) .{ }^{13} \mathbf{C} \mathbf{N M R}\left(101 \mathrm{MHz}, \mathrm{CDCl}_{3}\right) \delta$ 118.75, 77.67, 72.79, 70.85, 66.41, 60.15, 57.83, 24.17, 18.39, 16.19. HRMS (ESI) Calcd. for $\mathrm{C}_{10} \mathrm{H}_{11} \mathrm{NO}[\mathrm{M}+\mathrm{H}]^{+}$: 162.0841, Found: 162.0845.

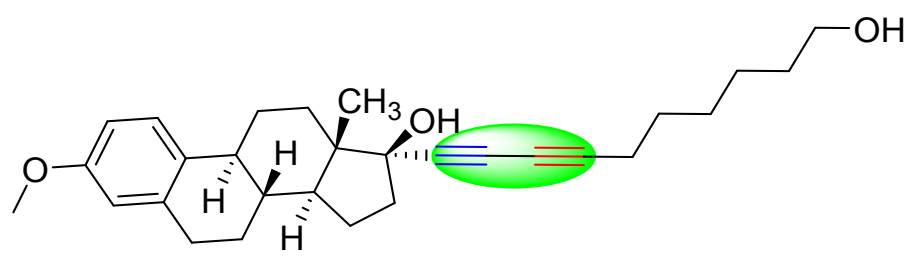

(8R,9S,13S,14S,17S)-17-(10-hydroxydeca-1,3-diyn-1-yl)-3-methoxy-13-methyl-

\section{7,8,9,11,12,13,14,15,16,17-decahydro-6H-cyclopenta[a]phenanthren-17-ol(4ac):}

Following general method. (8S,9S,14S,17S)-17-ethynyl-3-methoxy 7,8,9,11,12,13,14,15,16,17-decahydro-6H-cyclopenta[a]phenanthren-17-ol (1z; 40.4 mg, 0.13 mmol) and oct-7-yn-1-ol (1y, $32.8 \mathrm{mg}, 0.26 \mathrm{mmol})$ afforded the title compound as an green oil liquid (48.0 mg, 85\%) by using petroleum ether: ethyl acetate $(3: 1)$ as the column eluent; ${ }^{1} \mathbf{H}$ NMR $\left(400 \mathrm{MHz}, \mathrm{CDCl}_{3}\right) \delta 7.20(\mathrm{~d}, J=8.6 \mathrm{~Hz}, 1 \mathrm{H}), 6.71(\mathrm{dd}, J=8.6,2.8 \mathrm{~Hz}, 1 \mathrm{H}), 6.63(\mathrm{~d}, J$ $=2.7 \mathrm{~Hz}, 1 \mathrm{H}), 3.78(\mathrm{~s}, 3 \mathrm{H}), 3.64(\mathrm{t}, J=6.6 \mathrm{~Hz}, 2 \mathrm{H}), 2.85(\mathrm{dd}, J=6.7,3.4 \mathrm{~Hz}, 2 \mathrm{H}), 2.40-2.21$ (m, 5H), 2.02 (ddd, $J=13.9,11.9,3.9 \mathrm{~Hz}, 2 \mathrm{H}), 1.90-1.65$ (m, 7H), $1.62-1.53(\mathrm{~m}, 4 \mathrm{H}), 1.48$ $-1.35(\mathrm{~m}, 7 \mathrm{H}), 0.87(\mathrm{~s}, 3 \mathrm{H}) .{ }^{13} \mathbf{C}$ NMR $\left(101 \mathrm{MHz}, \mathrm{CDCl}_{3}\right) \delta 157.42,137.96,132.55,126.37$, 113.80, 111.50, 81.91, 80.47, 79.10, 70.99, 64.71, 62.85, 55.22, 49.76, 47.86, 43.42, 39.47, $38.96,33.04,32.54,29.84,28.65,28.14,27.22,26.43,25.25,22.92,19.32,12.79$. HRMS (ESI) Calcd. for $\mathrm{C}_{29} \mathrm{H}_{38} \mathrm{O}_{3}[\mathrm{M}+\mathrm{H}]^{+}: 435.2821$, Found: 435.2830 .

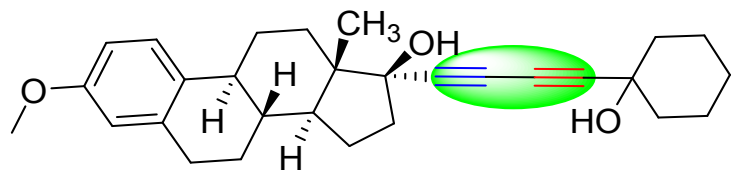


methyl-7,8,9,11,12,13,14,15,16,17-decahydro-6H-cyclopenta[a]phenanthren-17-ol(4ad):

Following general method. (8S,9S,14S,17S)-17-ethynyl-3-methoxy7,8,9,11,12,13,14,15,16,17-decahydro-6H-cyclopenta[a]phenanthren-17-ol (1z; 40.4 mg, 0.13 $\mathrm{mmol}$ ) and 1-ethynylcyclohexan-1-ol (1w, $32.3 \mathrm{mg}, 0.26 \mathrm{mmol})$ afforded the title compound as an white solid $(47.8 \mathrm{mg}, 85 \%)$ by using petroleum ether: ethyl acetate $(20: 1)$ as the column eluent; ${ }^{1} \mathbf{H}$ NMR $\left(600 \mathrm{MHz}, \mathrm{CDCl}_{3}\right) \delta 7.20(\mathrm{~d}, J=8.6 \mathrm{~Hz}, 1 \mathrm{H}), 6.71(\mathrm{dt}, J=8.7,2.5 \mathrm{~Hz}, 1 \mathrm{H})$, $6.63(\mathrm{t}, J=2.1 \mathrm{~Hz}, 1 \mathrm{H}), 3.78(\mathrm{~s}, 3 \mathrm{H}), 2.85(\mathrm{dt}, J=6.9,4.6 \mathrm{~Hz}, 2 \mathrm{H}), 2.37$ (ddt, $J=13.8,5.6,3.7$ $\mathrm{Hz}, 2 \mathrm{H}), 2.26$ (s, 1H), $2.06-2.00(\mathrm{~m}, 2 \mathrm{H}), 1.87-1.39$ (m, 20H), 0.88 (s, 3H). ${ }^{13}$ C NMR (151 $\left.\mathrm{MHz}, \mathrm{CDCl}_{3}\right) \delta 157.45,137.94,132.47,126.37,113.81,111.52,82.81,80.68,80.61,77.25$, 77.04, 76.83, 70.26, 69.22, 68.35, 55.22, 49.82, 47.93, 43.40, 39.67, 39.46, 33.03, 29.83, 27.21, 26.40, 25.02, 23.12, 12.77. HRMS (ESI) Calcd. for $\mathrm{C}_{29} \mathrm{H}_{36} \mathrm{O}_{3}[\mathrm{M}+\mathrm{H}]^{+}$: 433.2664, Found: 433.2670.

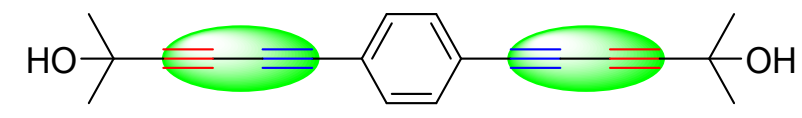

6,6'-(1,4-phenylene)bis(2-methylhexa-3,5-diyn-2-ol)(4ae): Following general method. 1,4diethynylbenzene (1; $10.1 \mathrm{mg}, 0.08 \mathrm{mmol})$ and 2-methylbut-3-yn-2-ol (1x, $26.9 \mathrm{mg}, 0.32 \mathrm{mmol})$ afforded the title compound as an pale yellow solid (13.5 $\mathrm{mg}, 58 \%$ ) by using petroleum ether: ethyl acetate (10:1) as the column eluent; ${ }^{1} \mathbf{H}$ NMR (600 MHz, $\left.\mathrm{CDCl}_{3}\right) \delta 7.41$ (s, 1H), 1.58 (s, 3H). ${ }^{13} \mathrm{C}$ NMR $\left(151 \mathrm{MHz}, \mathrm{CDCl}_{3}\right) \delta 132.43,122.38,88.05,77.97,75.61,66.86,65.78,31.08$. HRMS (ESI) Calcd. for $\mathrm{C}_{20} \mathrm{H}_{18} \mathrm{O}_{2}[\mathrm{M}+\mathrm{H}]^{+}:$291.1307, Found: 291.1311. 
c) ${ }^{1} \mathrm{H}$ and ${ }^{13} \mathrm{C}$ NMR spectra of products in oxidative homo-coupling

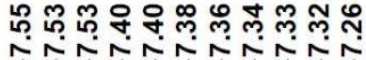

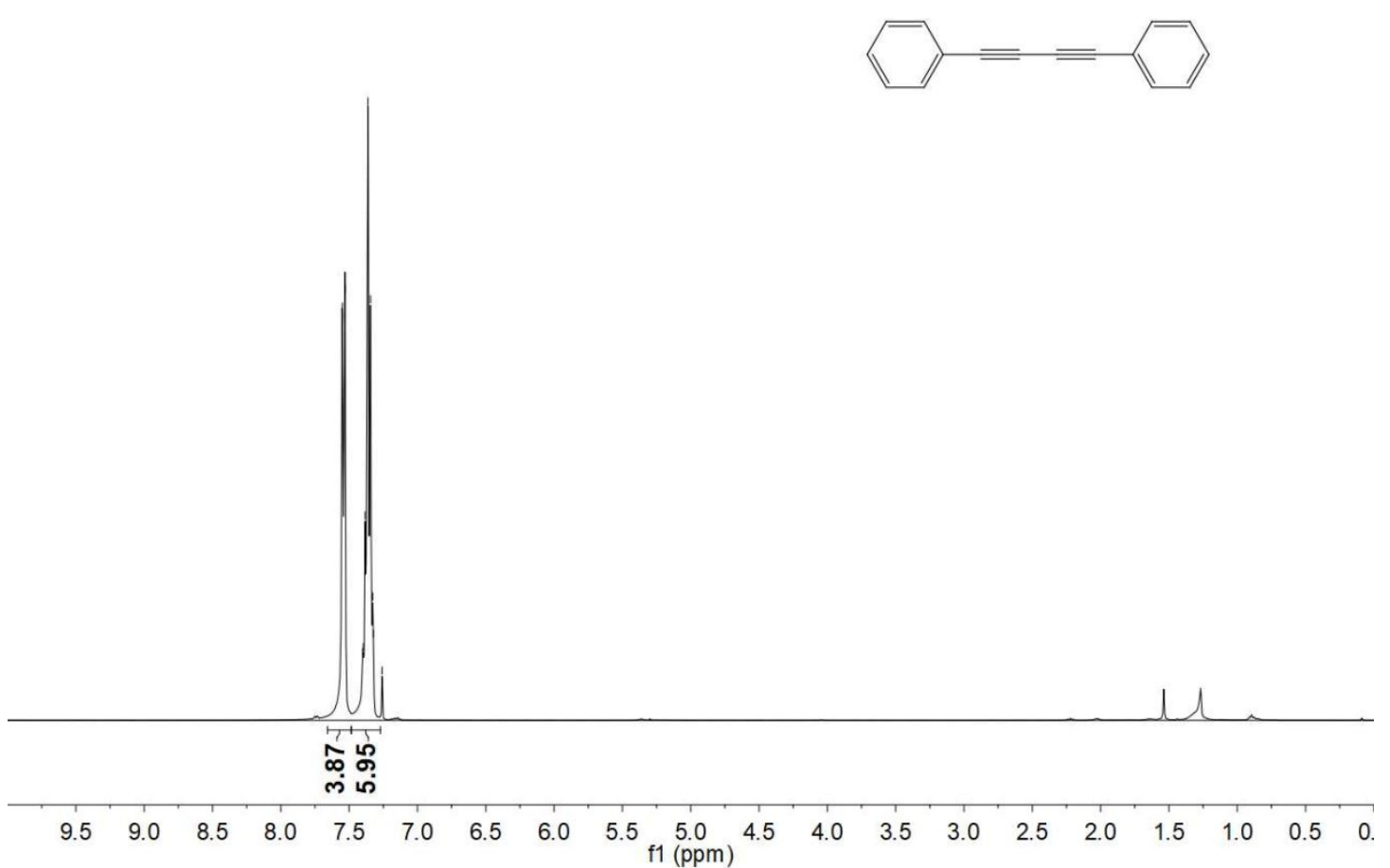

Figure S13. ${ }^{1} \mathrm{H}$ NMR spectroscopy of $\mathbf{3 a}$.

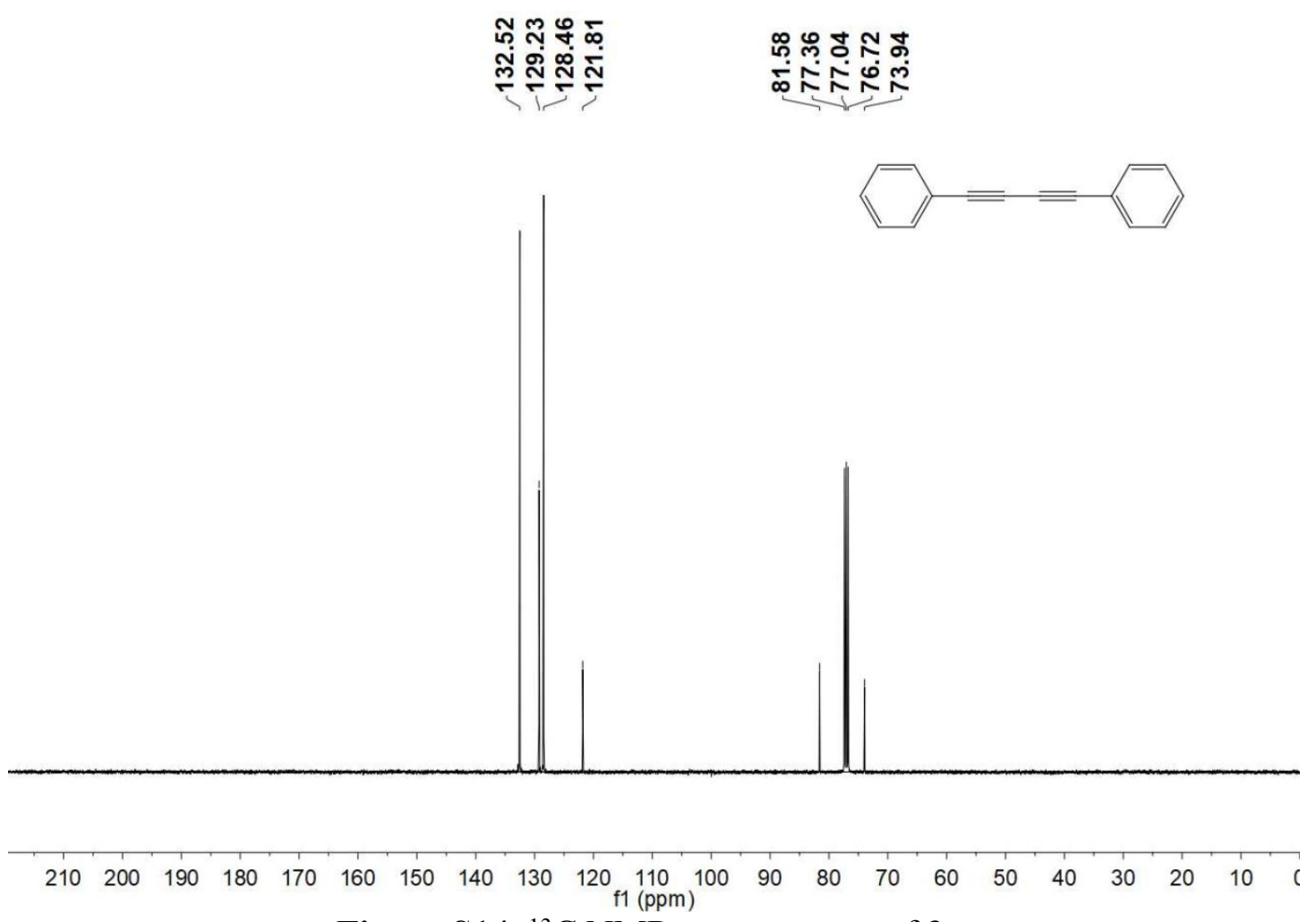

Figure S14. ${ }^{13} \mathrm{C}$ NMR spectroscopy of 3 a. 

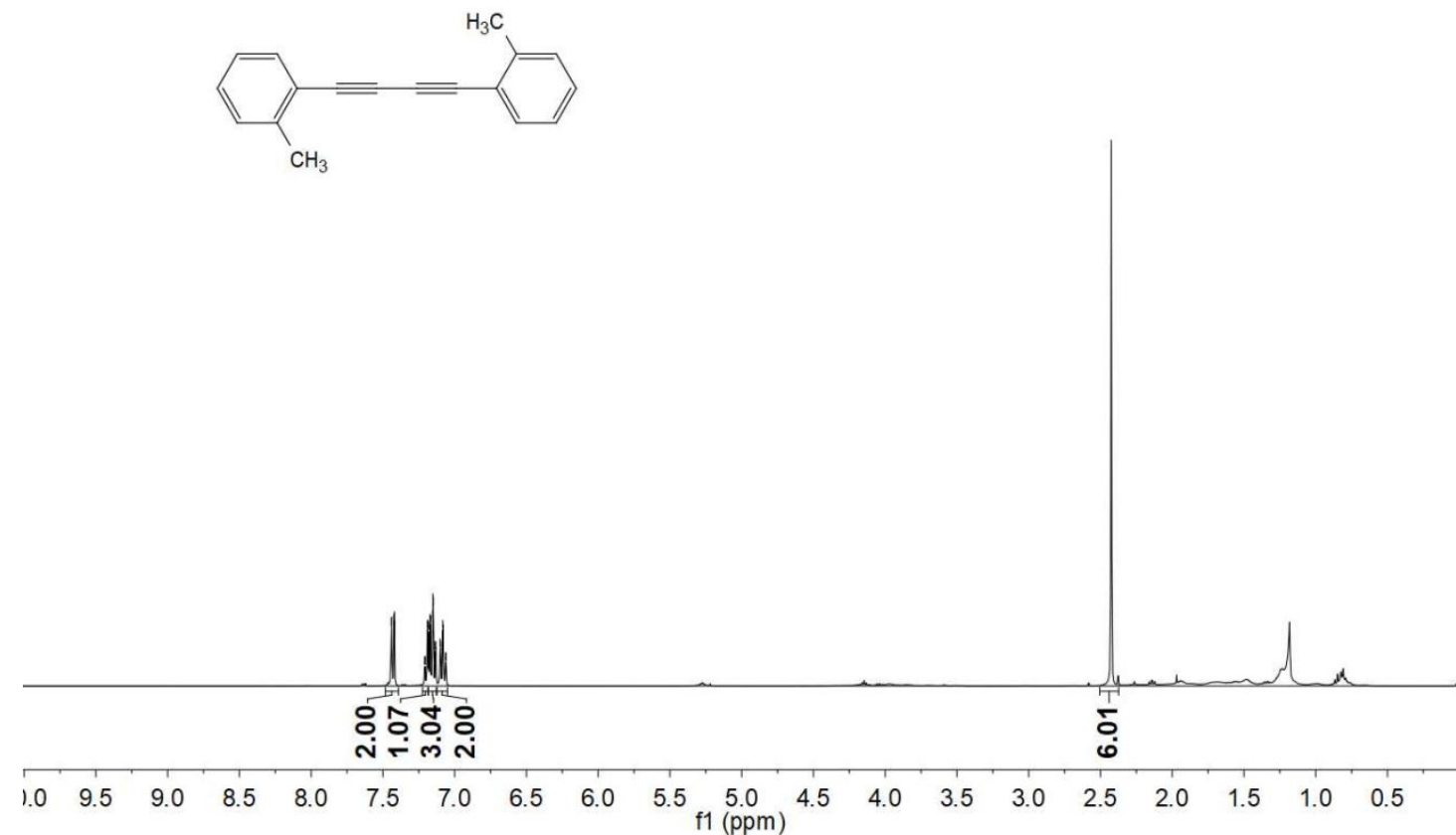

Figure S15. ${ }^{1} \mathrm{H}$ NMR spectroscopy of $\mathbf{3 b}$.

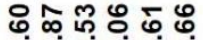

守宁突啇

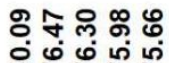

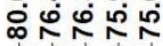

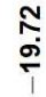
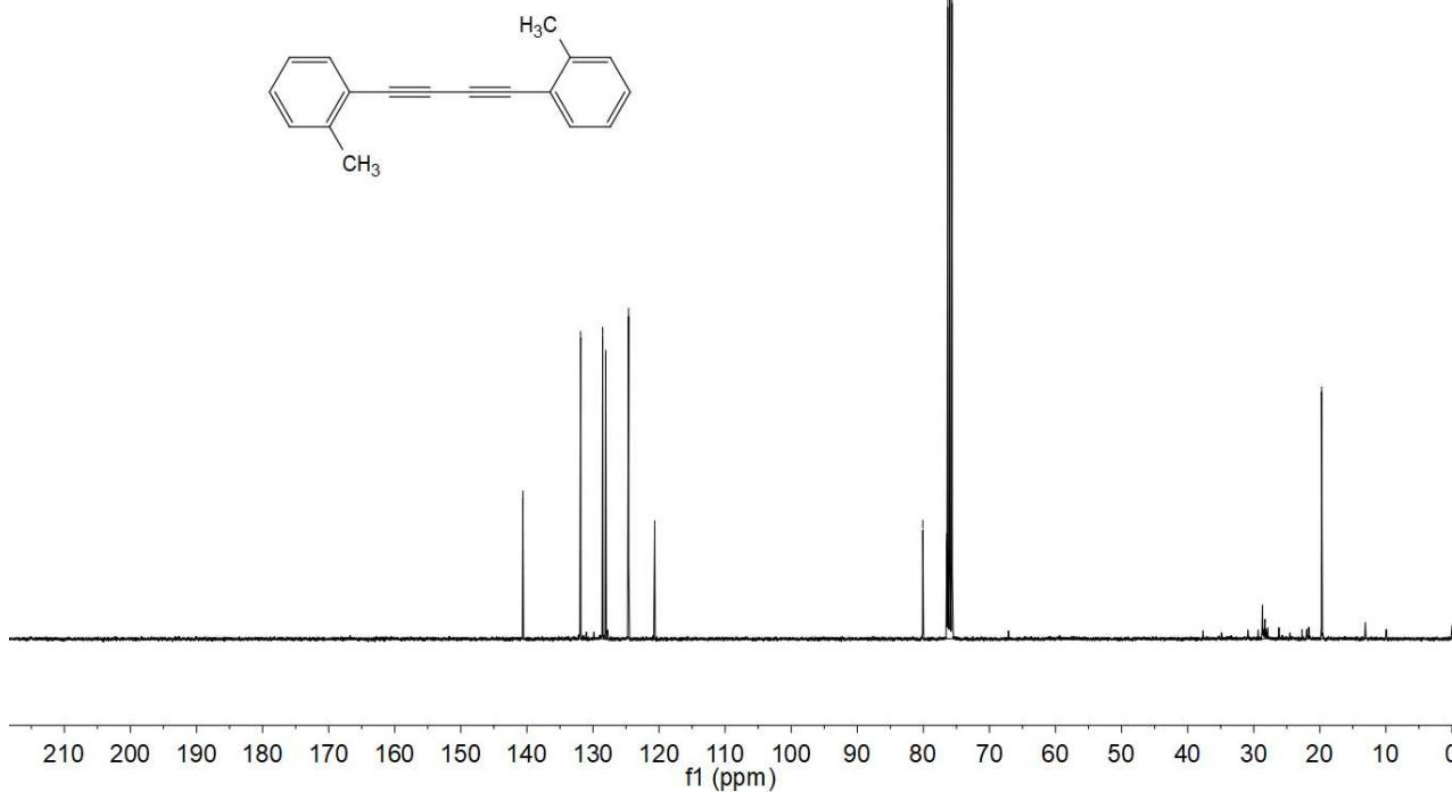

Figure S16. ${ }^{13} \mathrm{C}$ NMR spectroscopy of $\mathbf{3 b}$. 


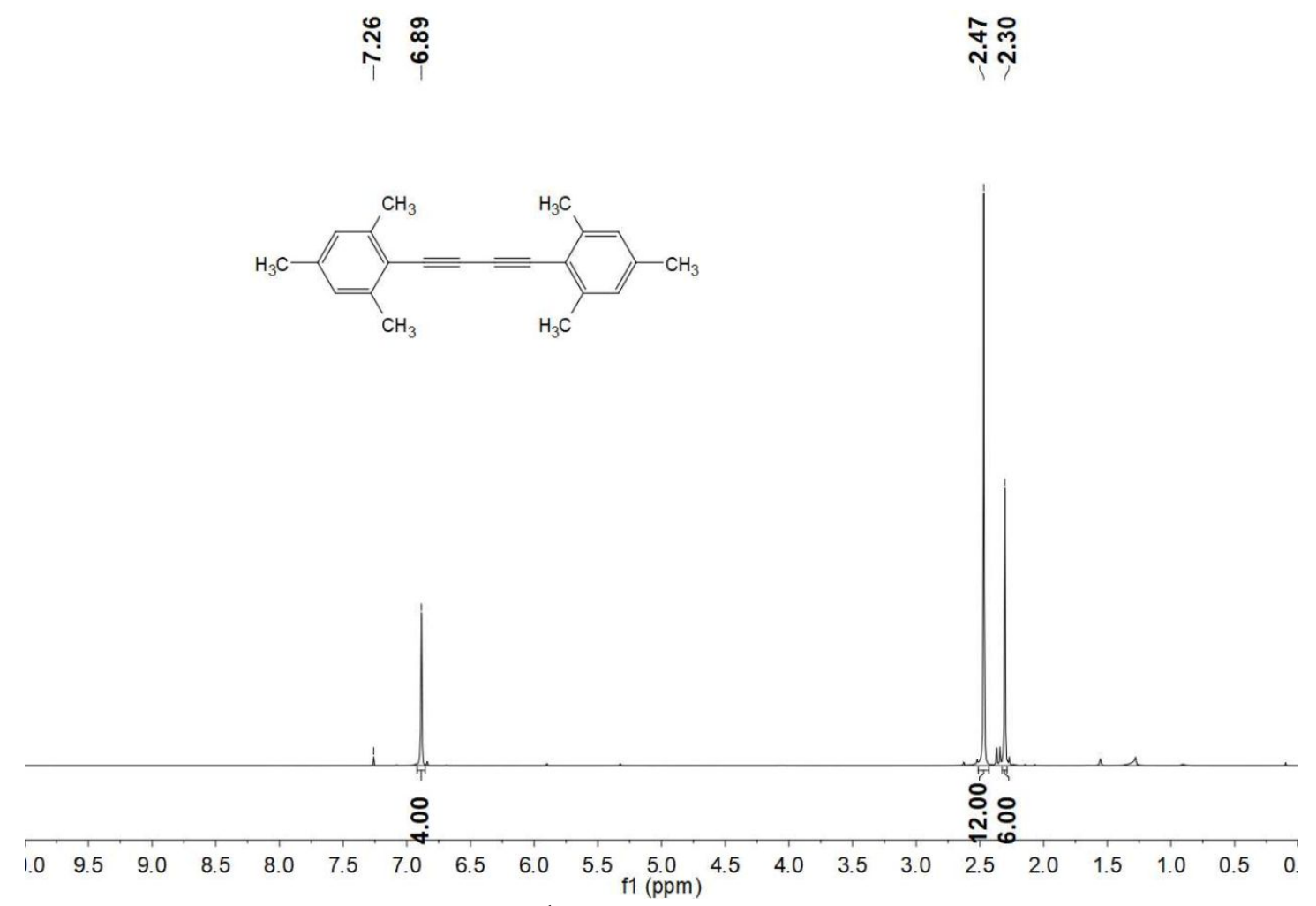

Figure S17. ${ }^{1} \mathrm{H}$ NMR spectroscopy of $\mathbf{3 c}$.

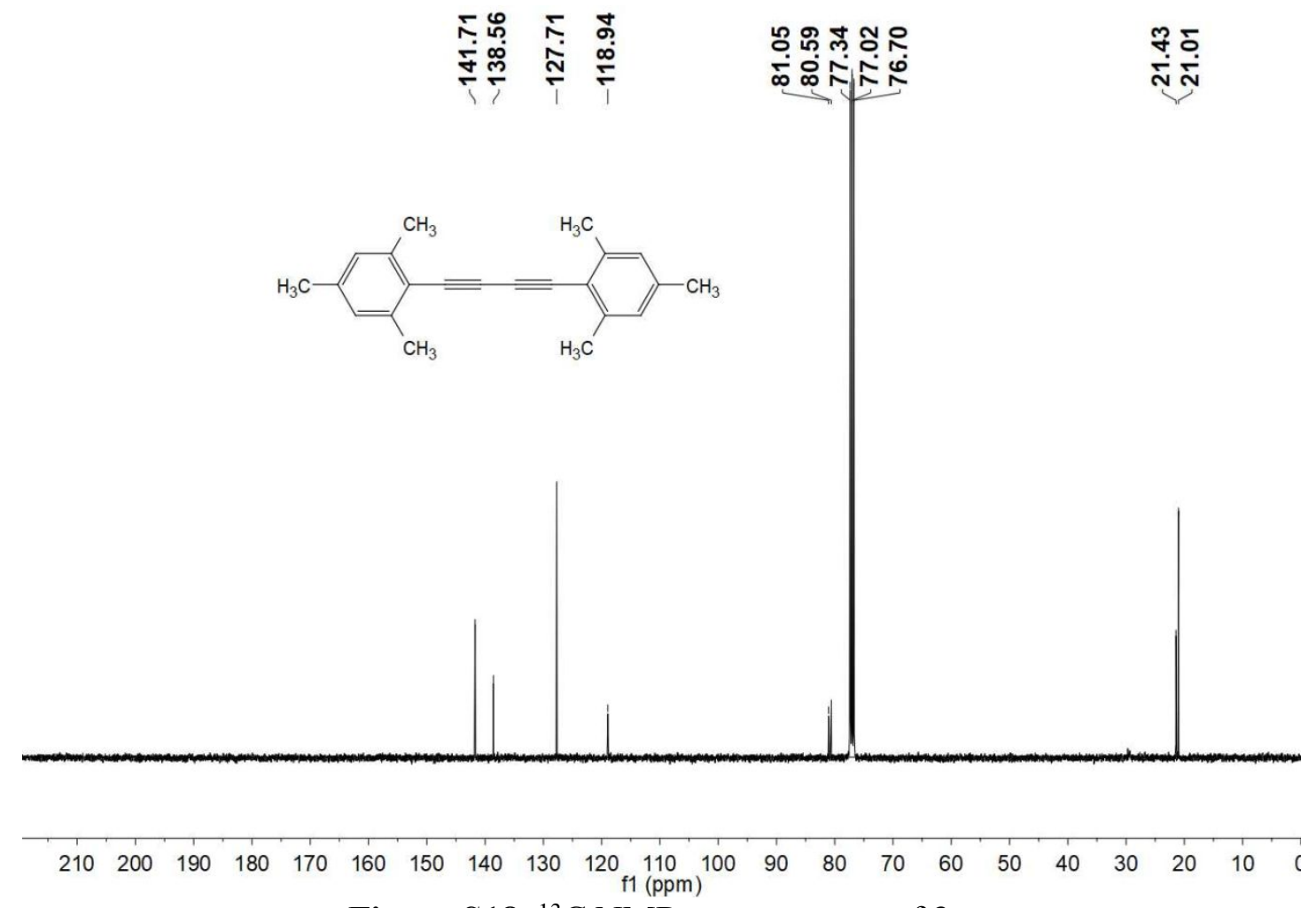

Figure S18. ${ }^{13} \mathrm{C}$ NMR spectroscopy of $\mathbf{3 c}$. 


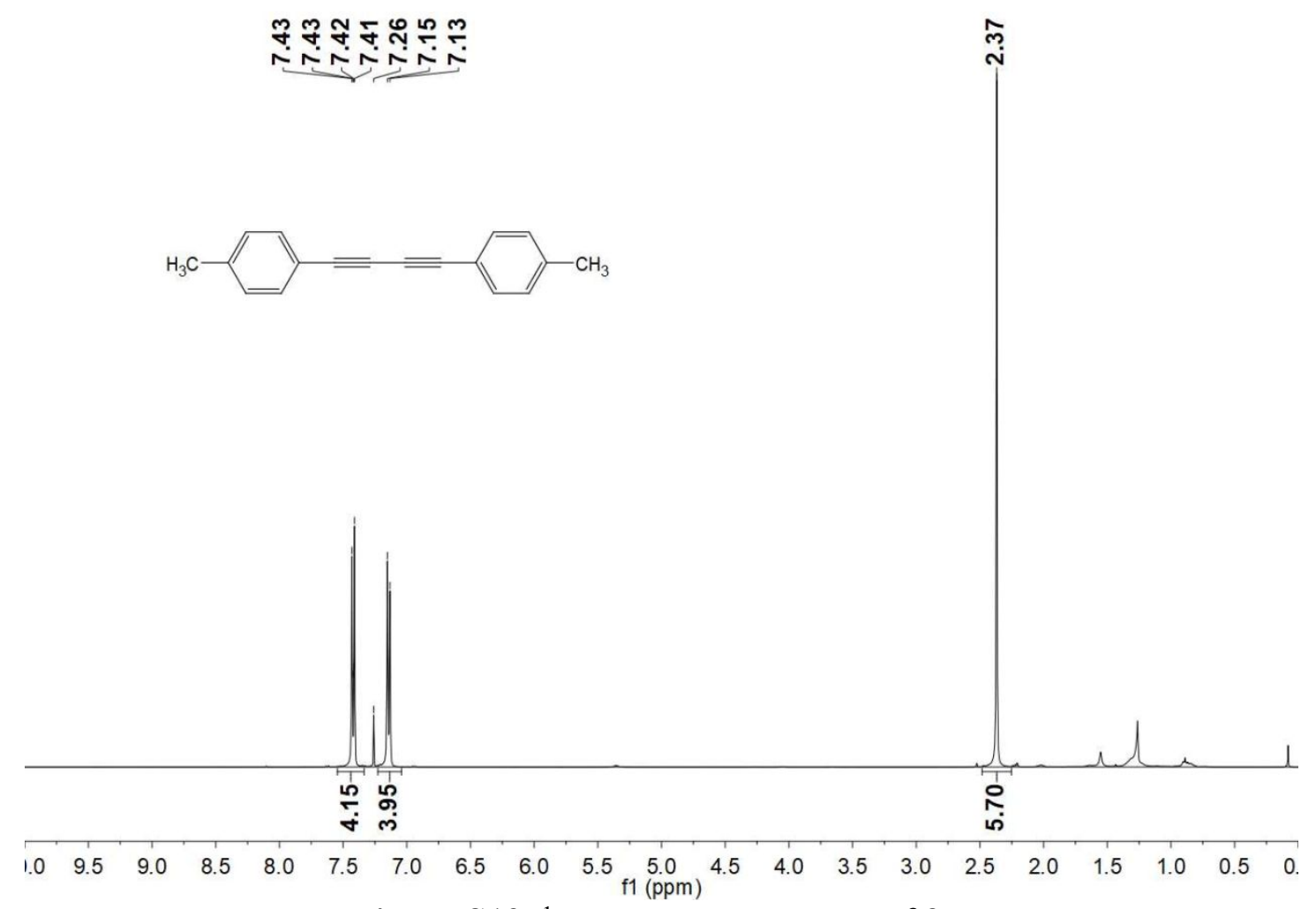

Figure S19. ${ }^{1} \mathrm{H}$ NMR spectroscopy of $\mathbf{3 d}$.

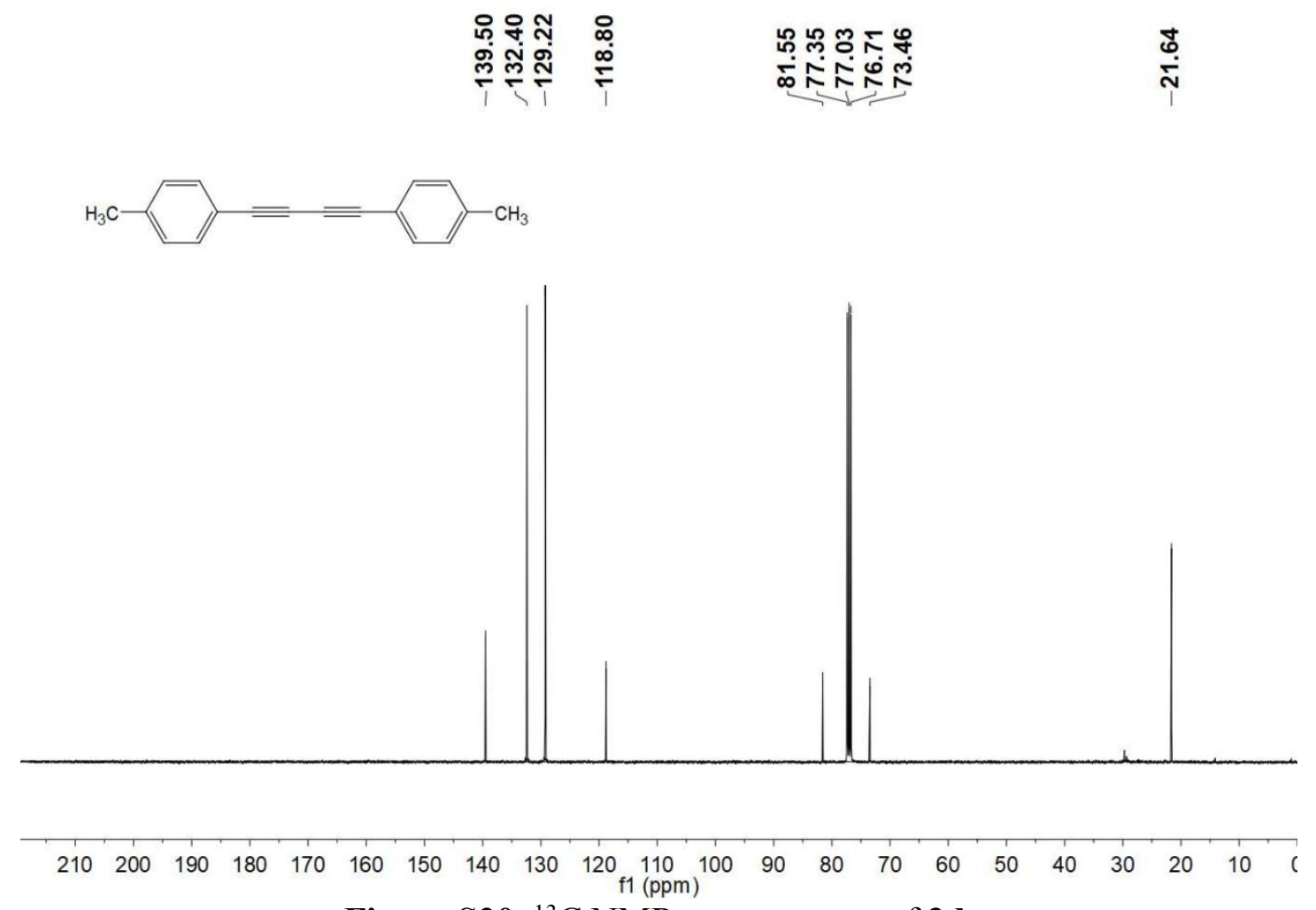

Figure S20. ${ }^{13} \mathrm{C}$ NMR spectroscopy of 3d. 

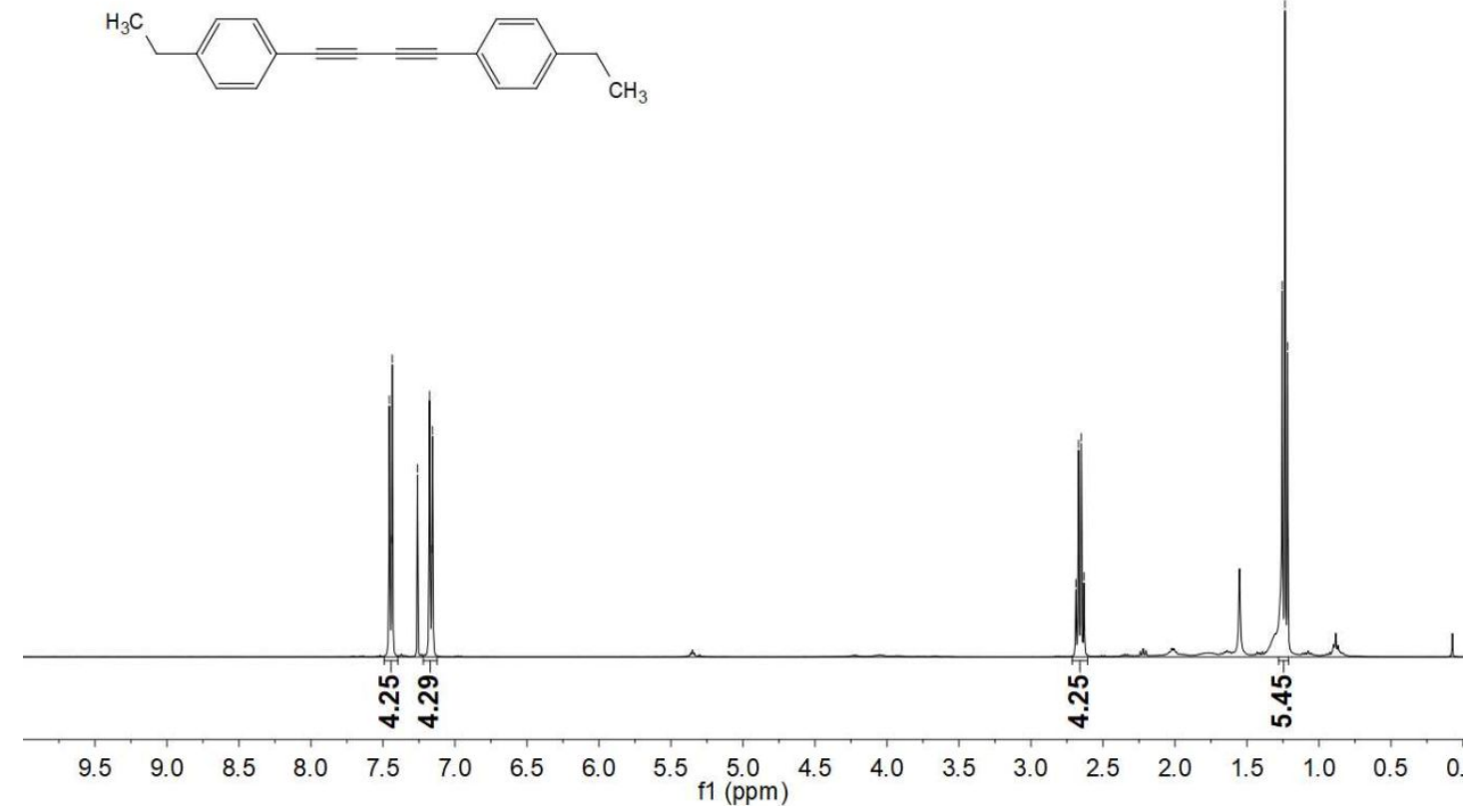

Figure S21. ${ }^{1} \mathrm{H}$ NMR spectroscopy of $\mathbf{3 e}$.

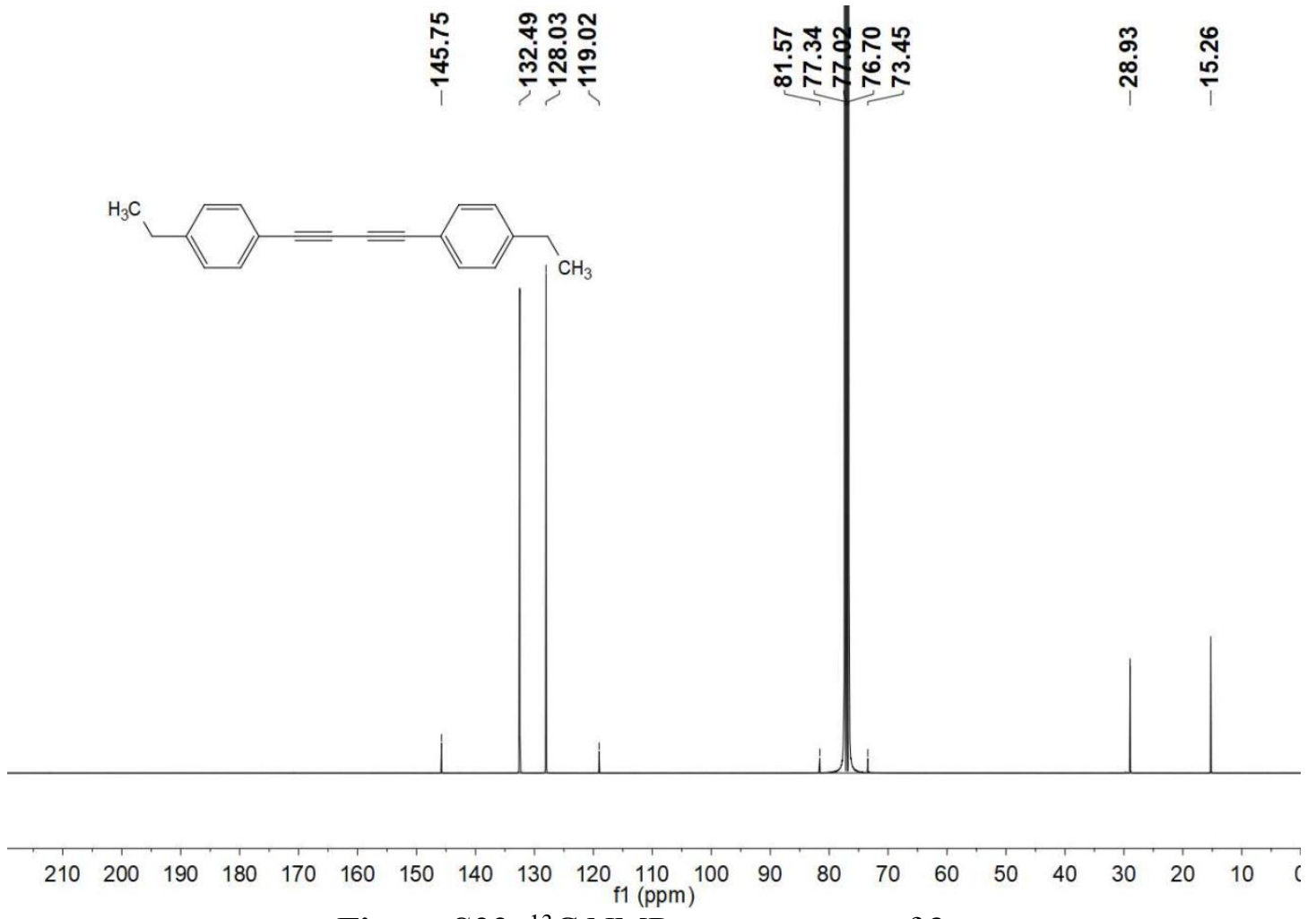

Figure S22. ${ }^{13} \mathrm{C}$ NMR spectroscopy of $\mathbf{3 e}$. 


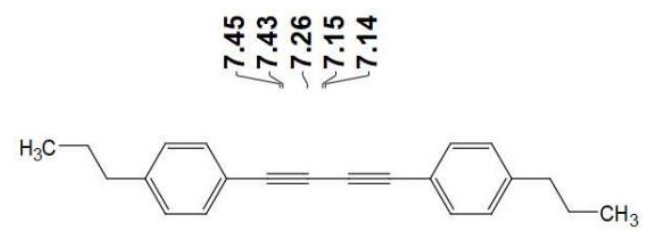

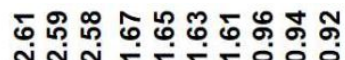

hintritiog

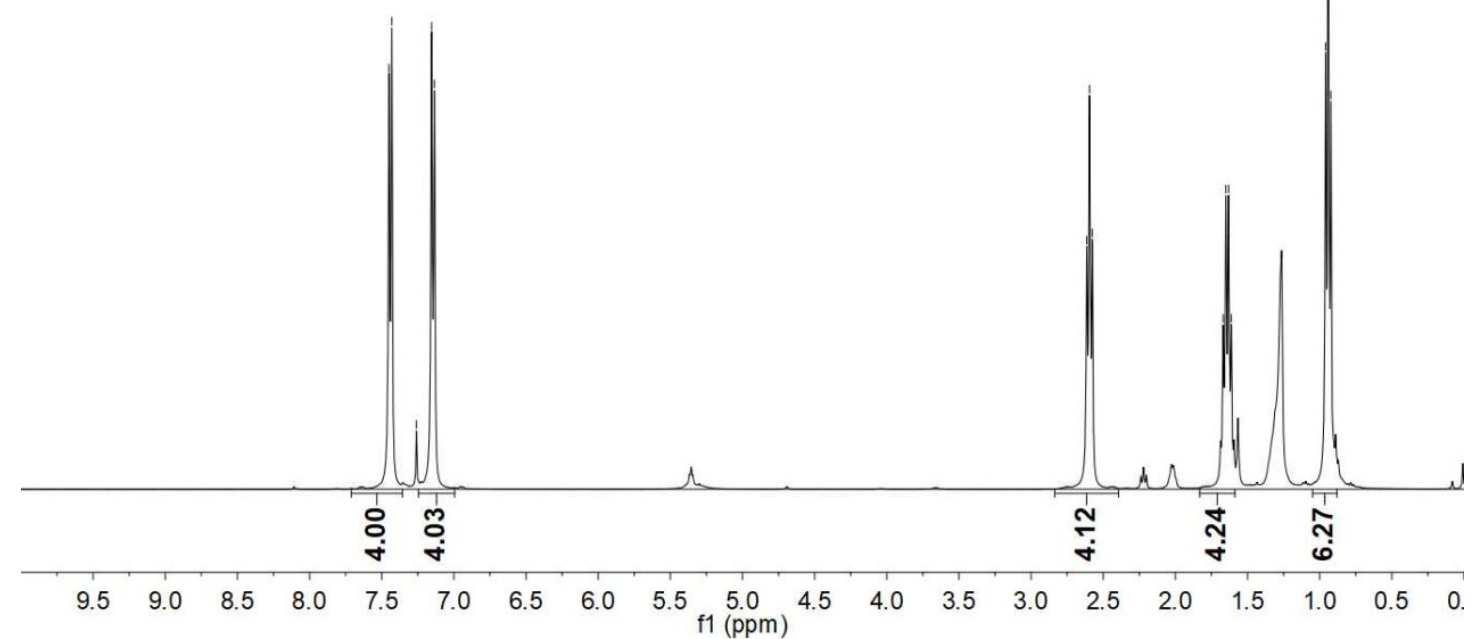

Figure S23. ${ }^{1} \mathrm{H}$ NMR spectroscopy of $\mathbf{3 f}$.

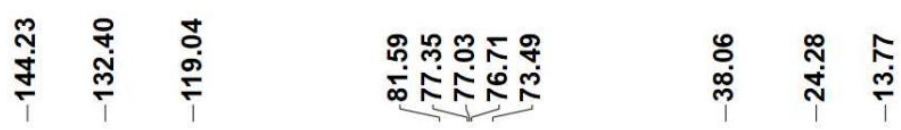
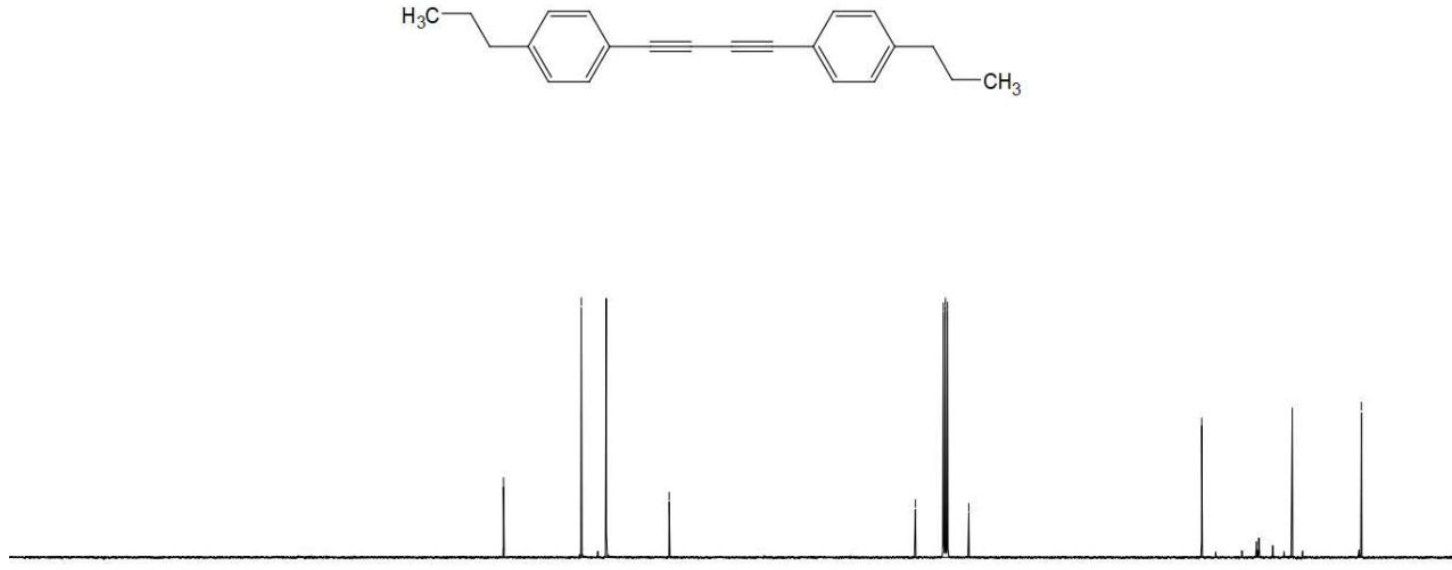

$\begin{array}{lllllllllllllllllllll}210 & 200 & 190 & 180 & 170 & 160 & 150 & 140 & 130 & 120 & 110 & 100 & 90 & 80 & 70 & 60 & 50 & 40 & 30 & 20 & 10\end{array}$

Figure S24. ${ }^{13} \mathrm{C}$ NMR spectroscopy of $\mathbf{3 f}$. 


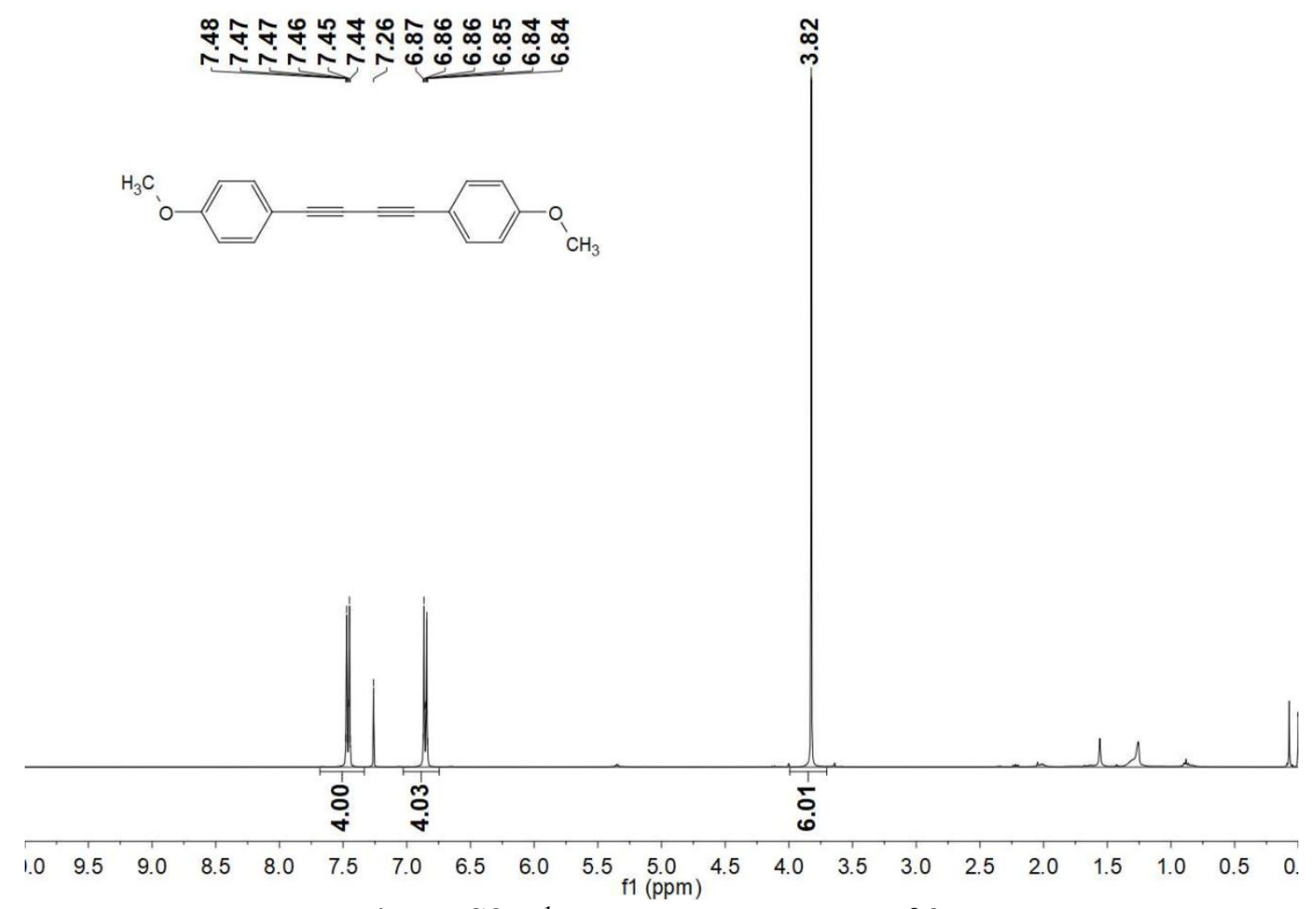

Figure S25. ${ }^{1} \mathrm{H}$ NMR spectroscopy of $\mathbf{3 g}$.

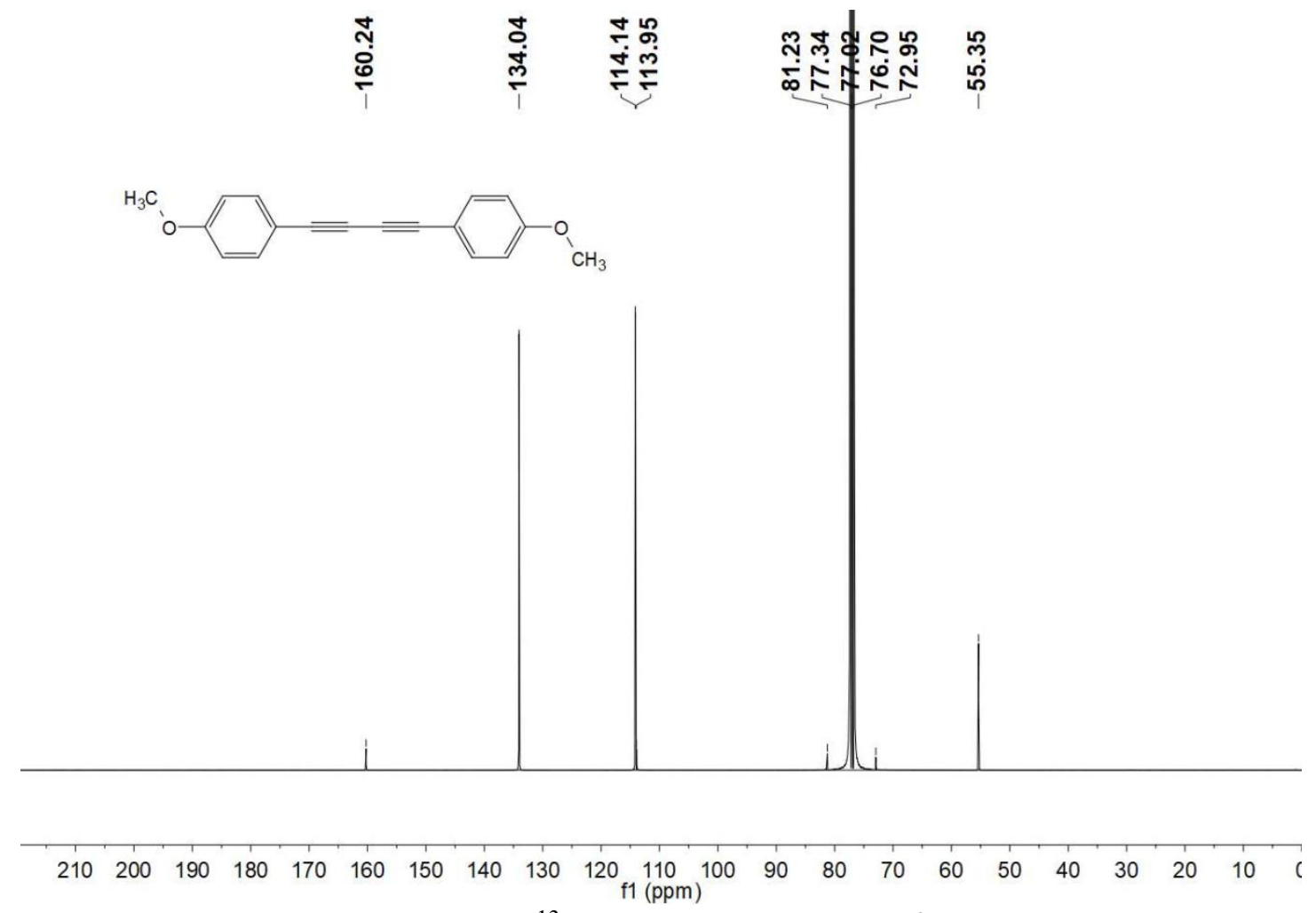

Figure S26. ${ }^{13} \mathrm{C}$ NMR spectroscopy of $\mathbf{3 g}$. 


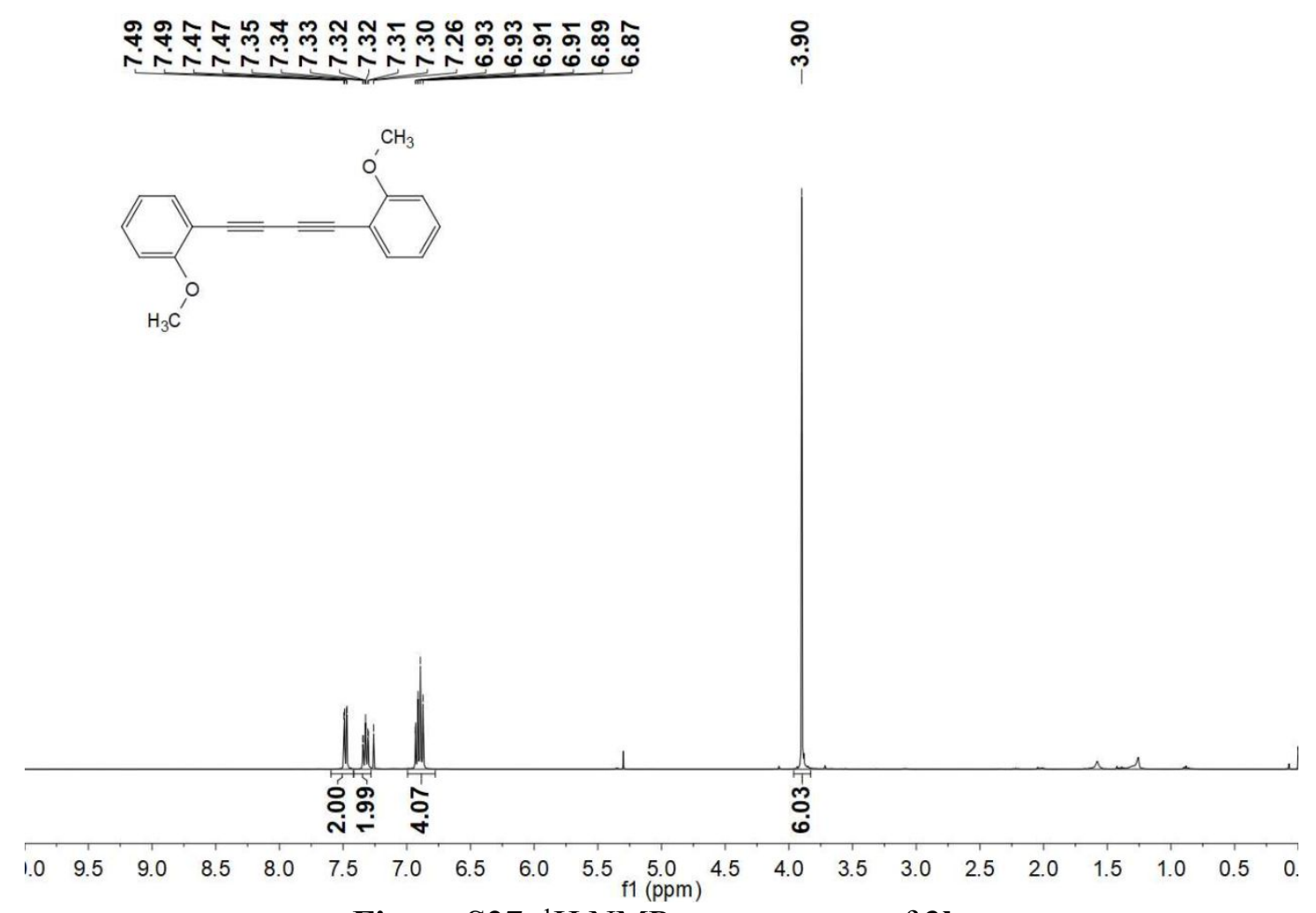

Figure S27. ${ }^{1} \mathrm{H}$ NMR spectroscopy of $\mathbf{3 h}$.

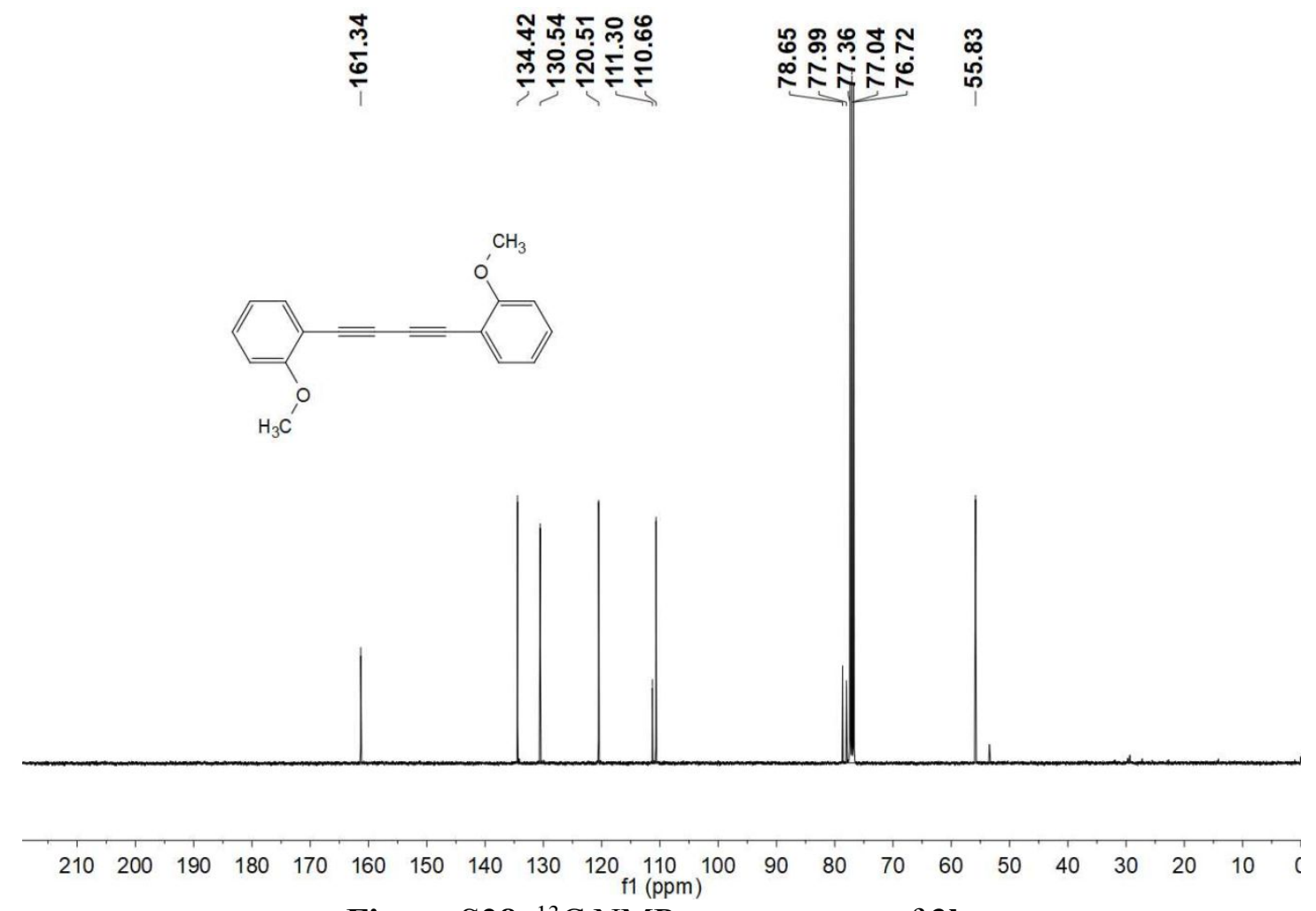

Figure S28. ${ }^{13} \mathrm{C}$ NMR spectroscopy of $\mathbf{3 h}$. 


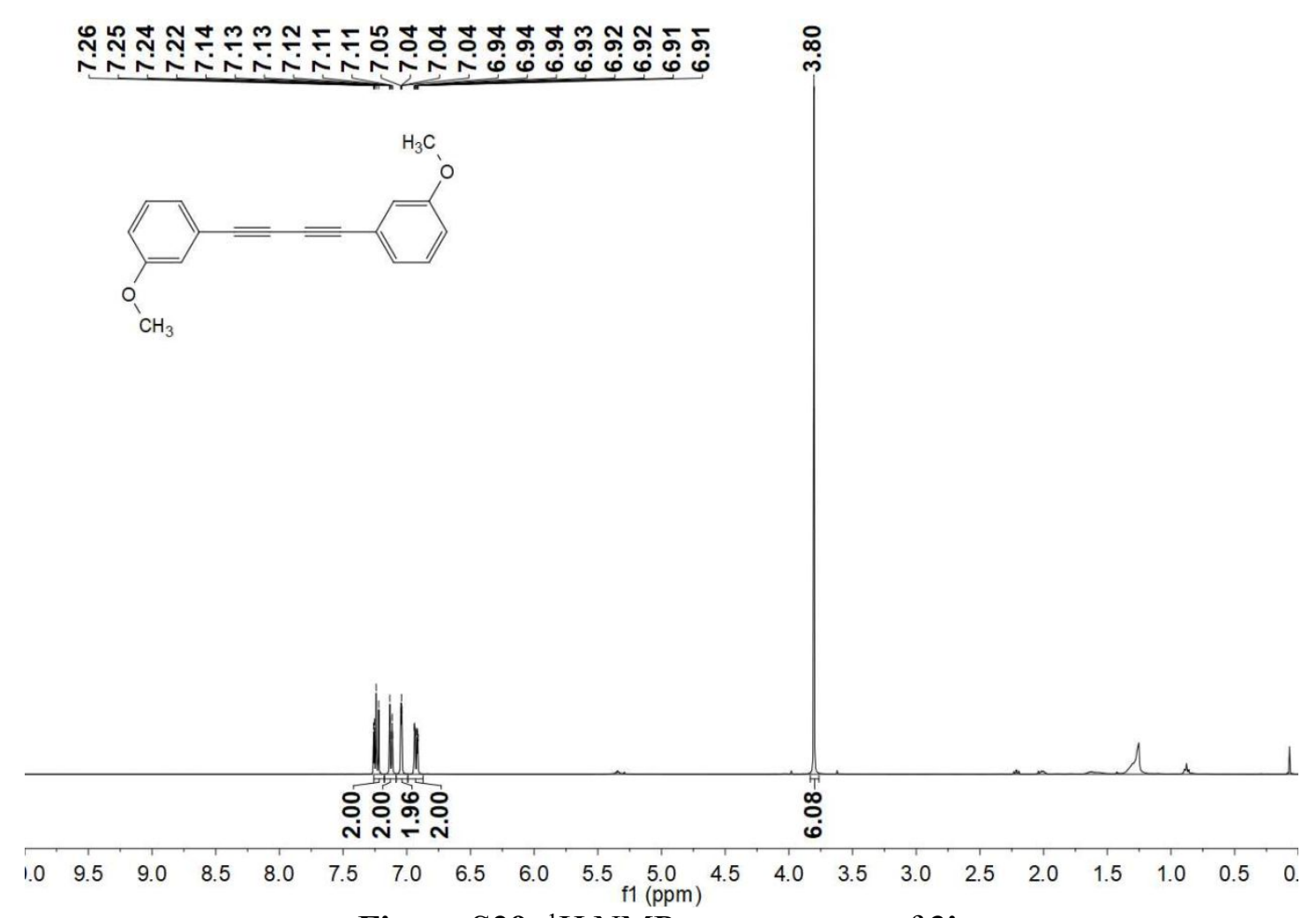

Figure S29. ${ }^{1} \mathrm{H}$ NMR spectroscopy of $\mathbf{3 i}$.

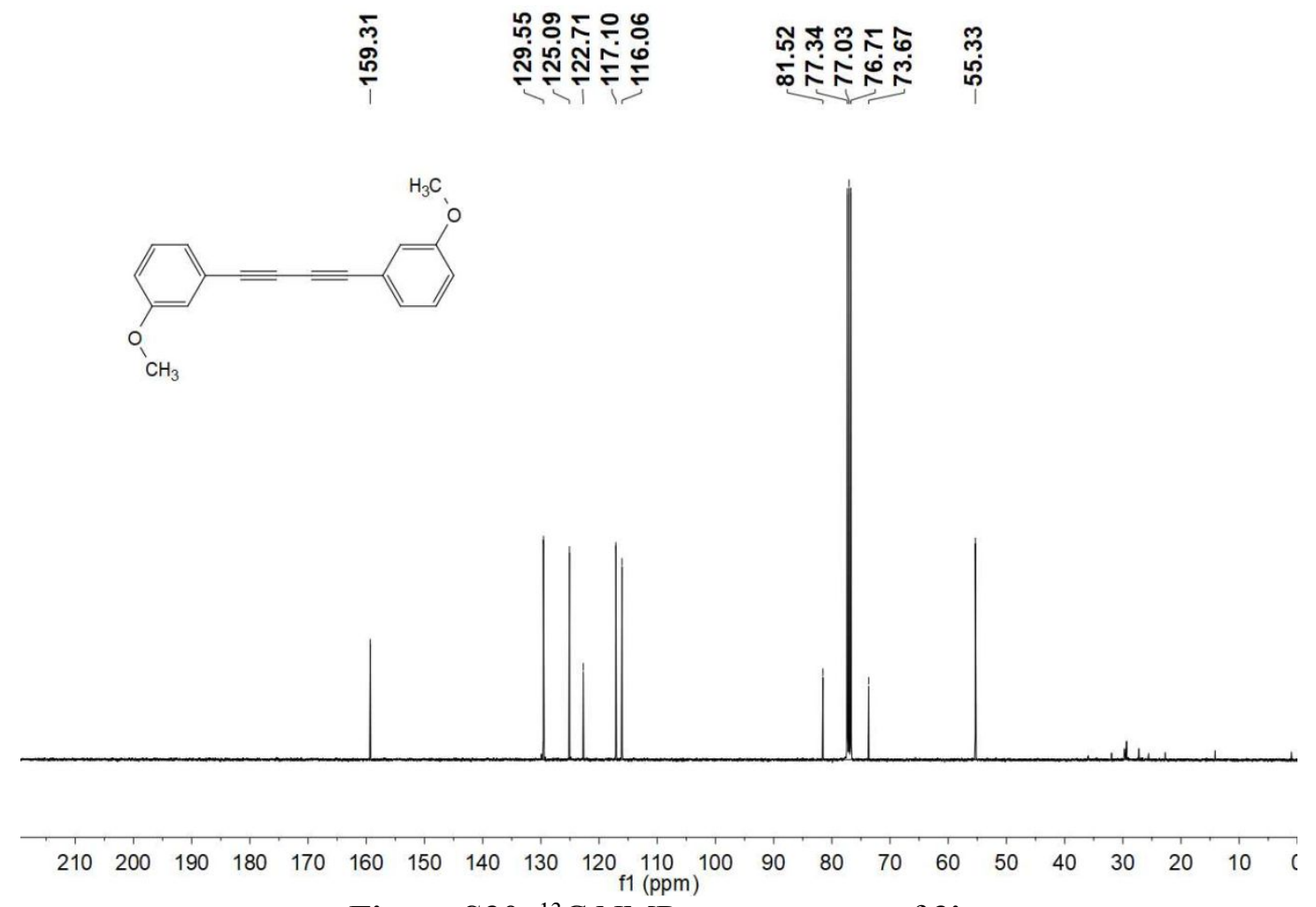

Figure S30. ${ }^{13} \mathrm{C}$ NMR spectroscopy of $\mathbf{3 i}$. 


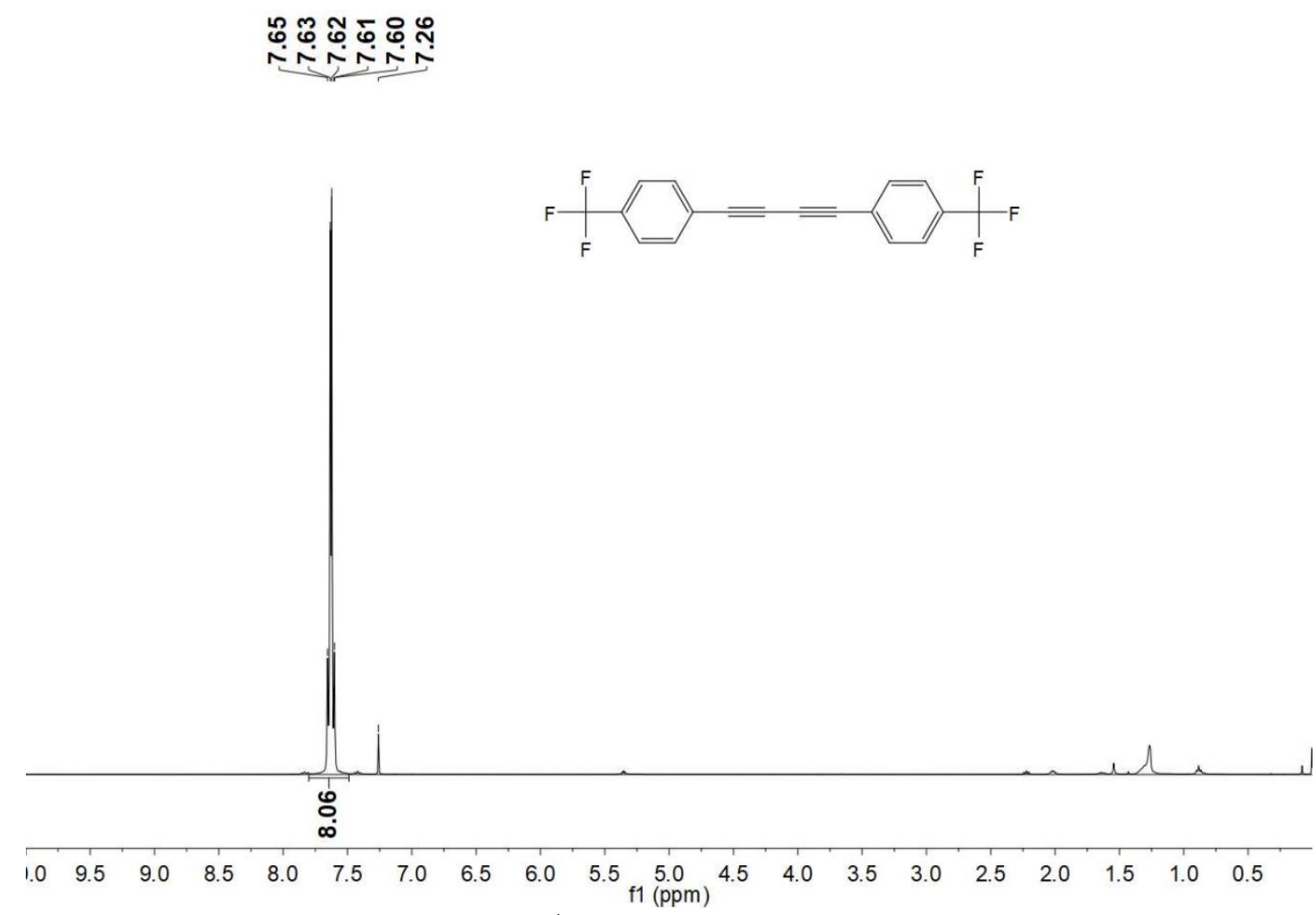

Figure S31. ${ }^{1} \mathrm{H}$ NMR spectroscopy of $\mathbf{3 j}$.

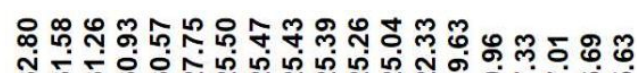

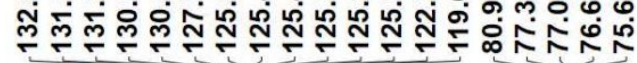

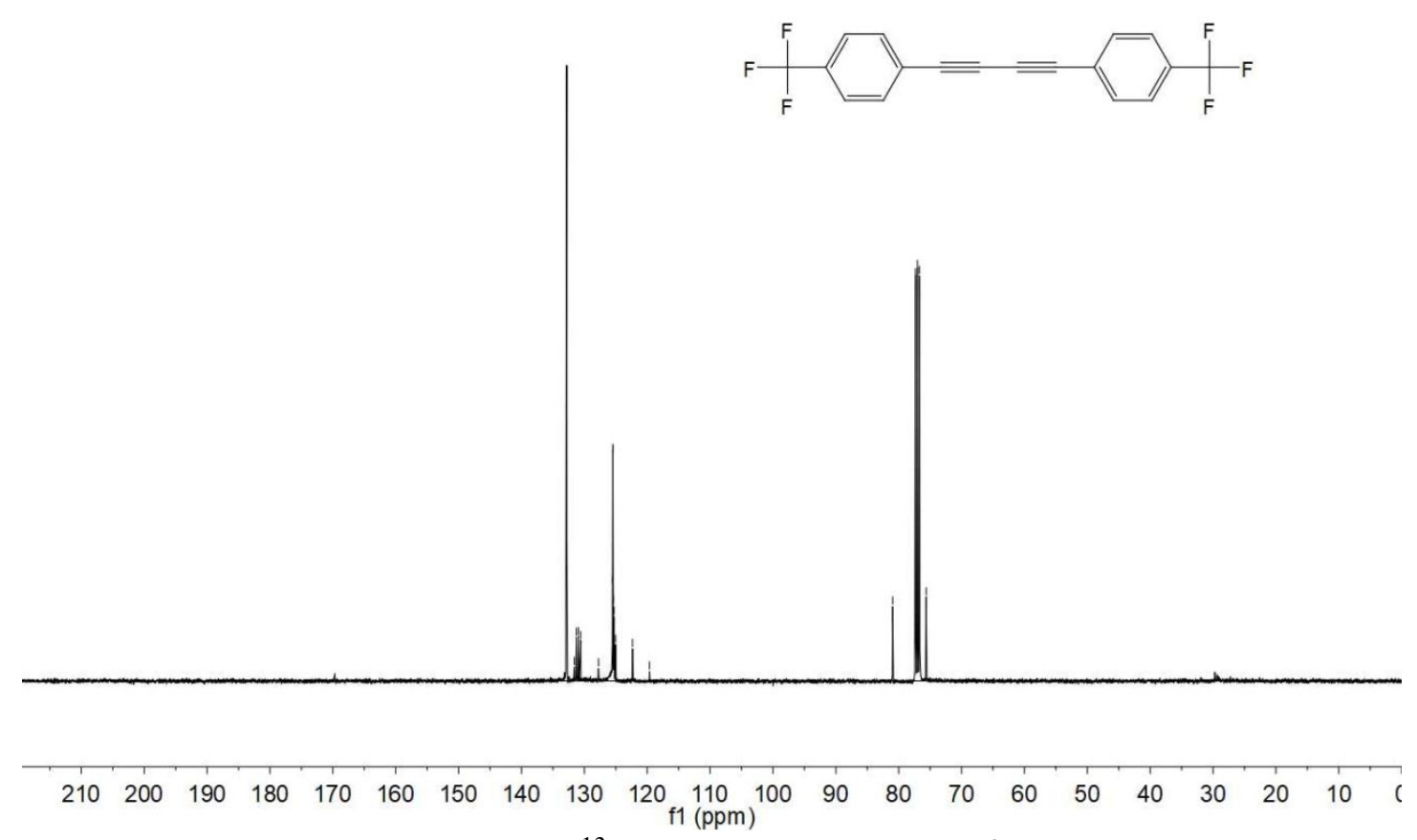

Figure S32. ${ }^{13} \mathrm{C}$ NMR spectroscopy of $\mathbf{3 j}$. 


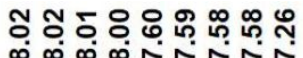
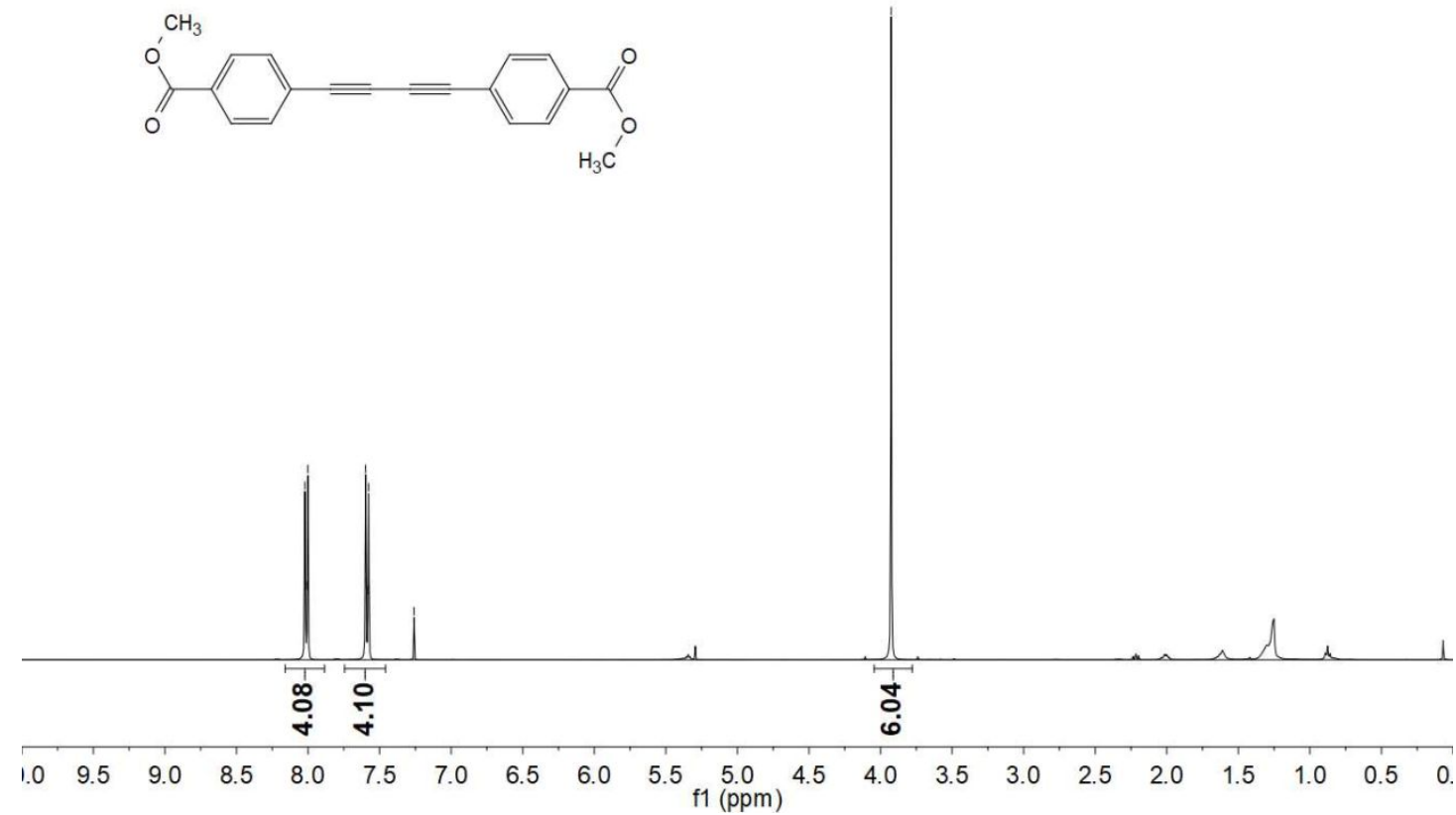

Figure S33. ${ }^{1} \mathrm{H}$ NMR spectroscopy of $\mathbf{3 k}$.

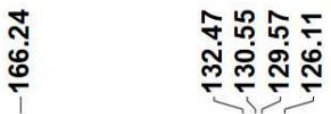

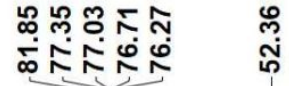
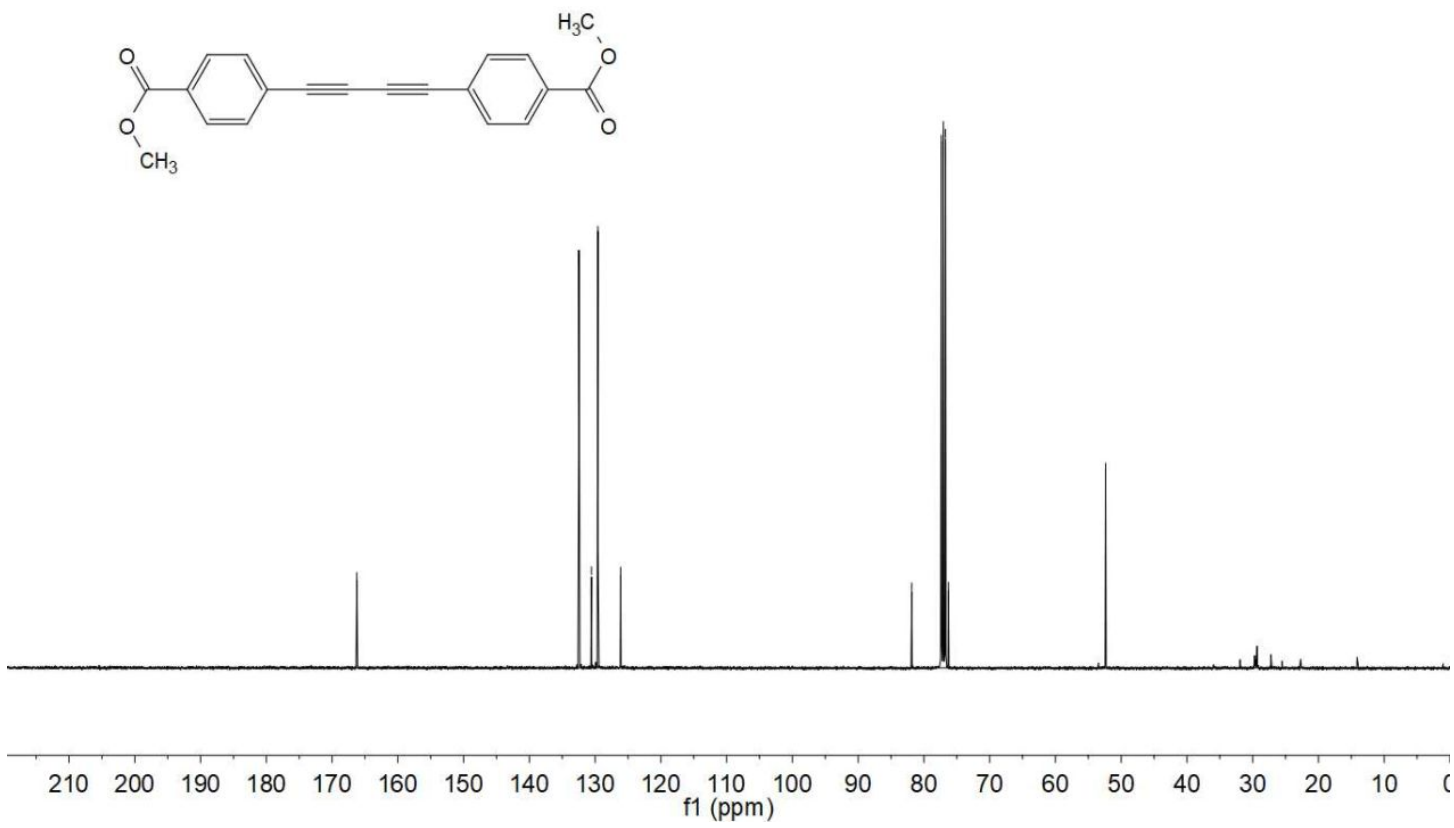

Figure S34. ${ }^{13} \mathrm{C}$ NMR spectroscopy of $3 \mathbf{k}$. 

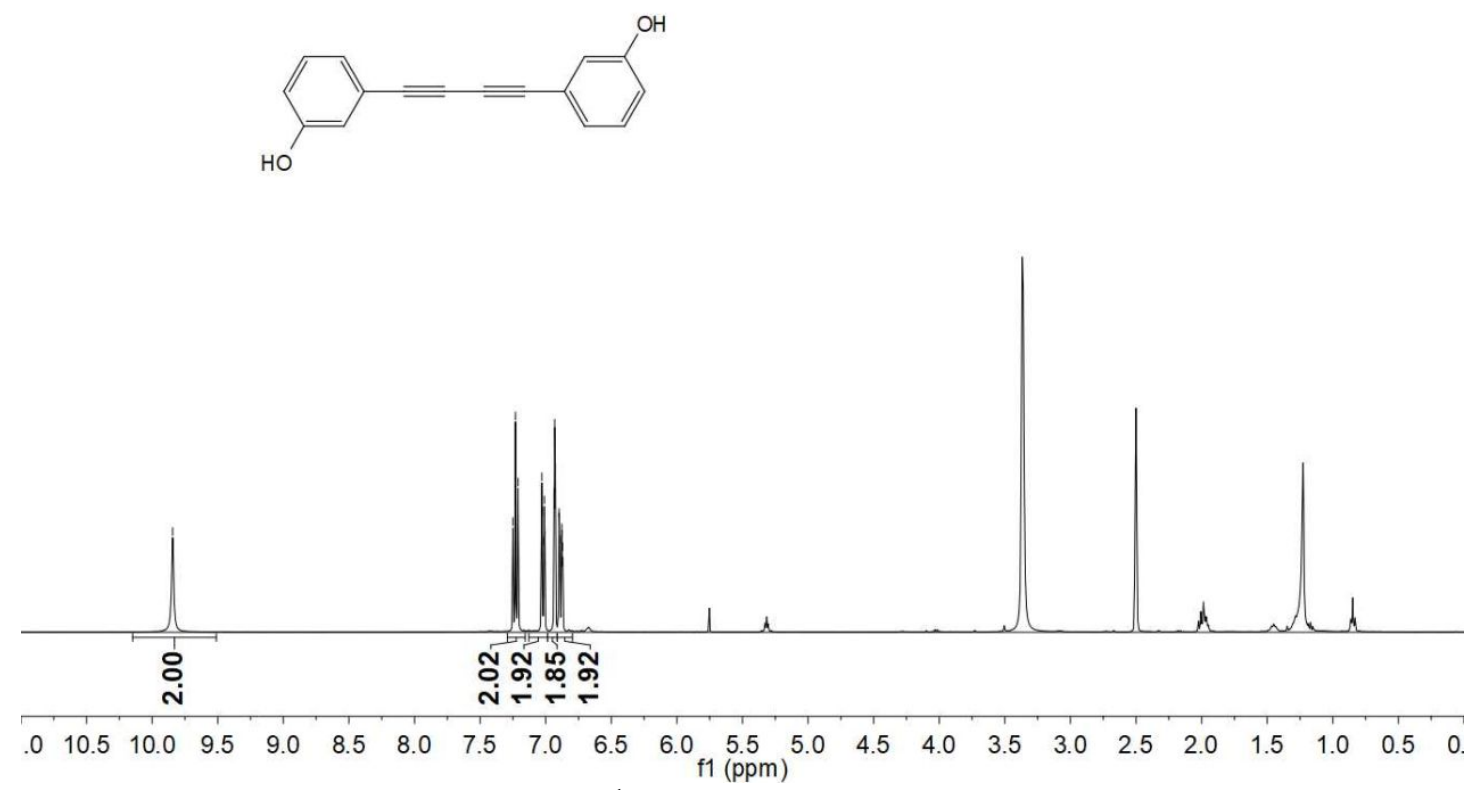

Figure S35. ${ }^{1} \mathrm{H}$ NMR spectroscopy of 31 .

\begin{tabular}{|c|c|c|c|c|}
\hline $\begin{array}{l}\stackrel{\infty}{\infty} \\
\stackrel{1}{n}\end{array}$ & 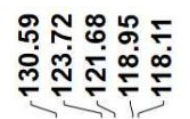 & 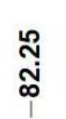 & $\stackrel{\substack{+i}}{i}$ & 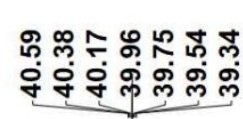 \\
\hline
\end{tabular}
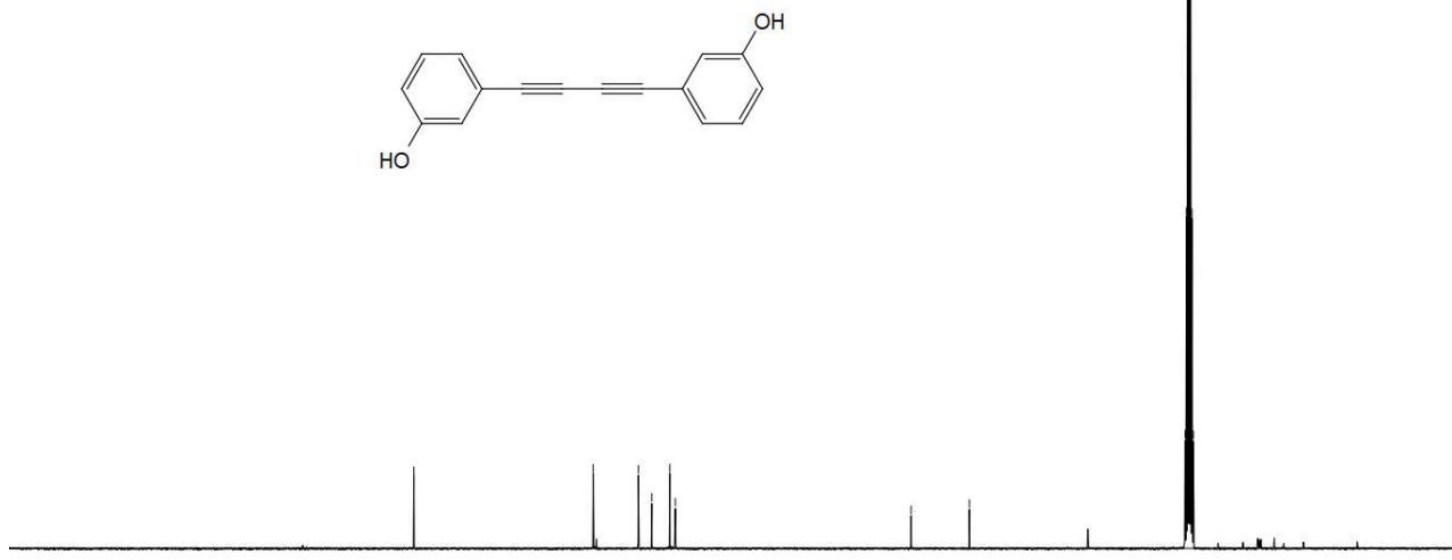

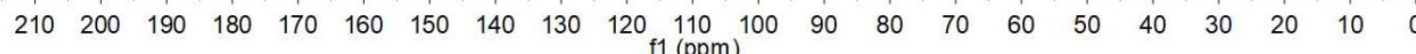

Figure S36. ${ }^{13} \mathrm{C}$ NMR spectroscopy of 31 . 

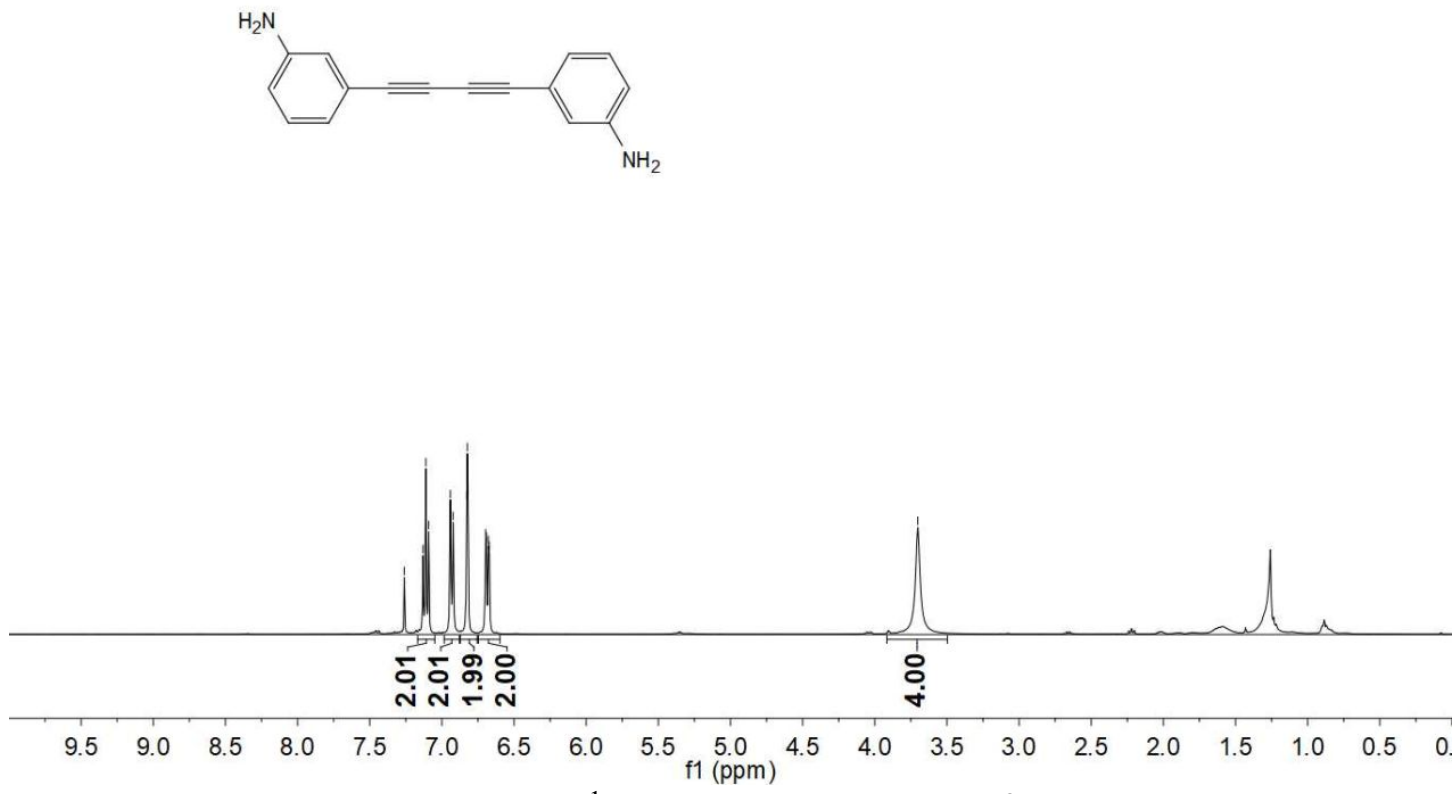

Figure S37. ${ }^{1} \mathrm{H}$ NMR spectroscopy of $\mathbf{3 m}$.

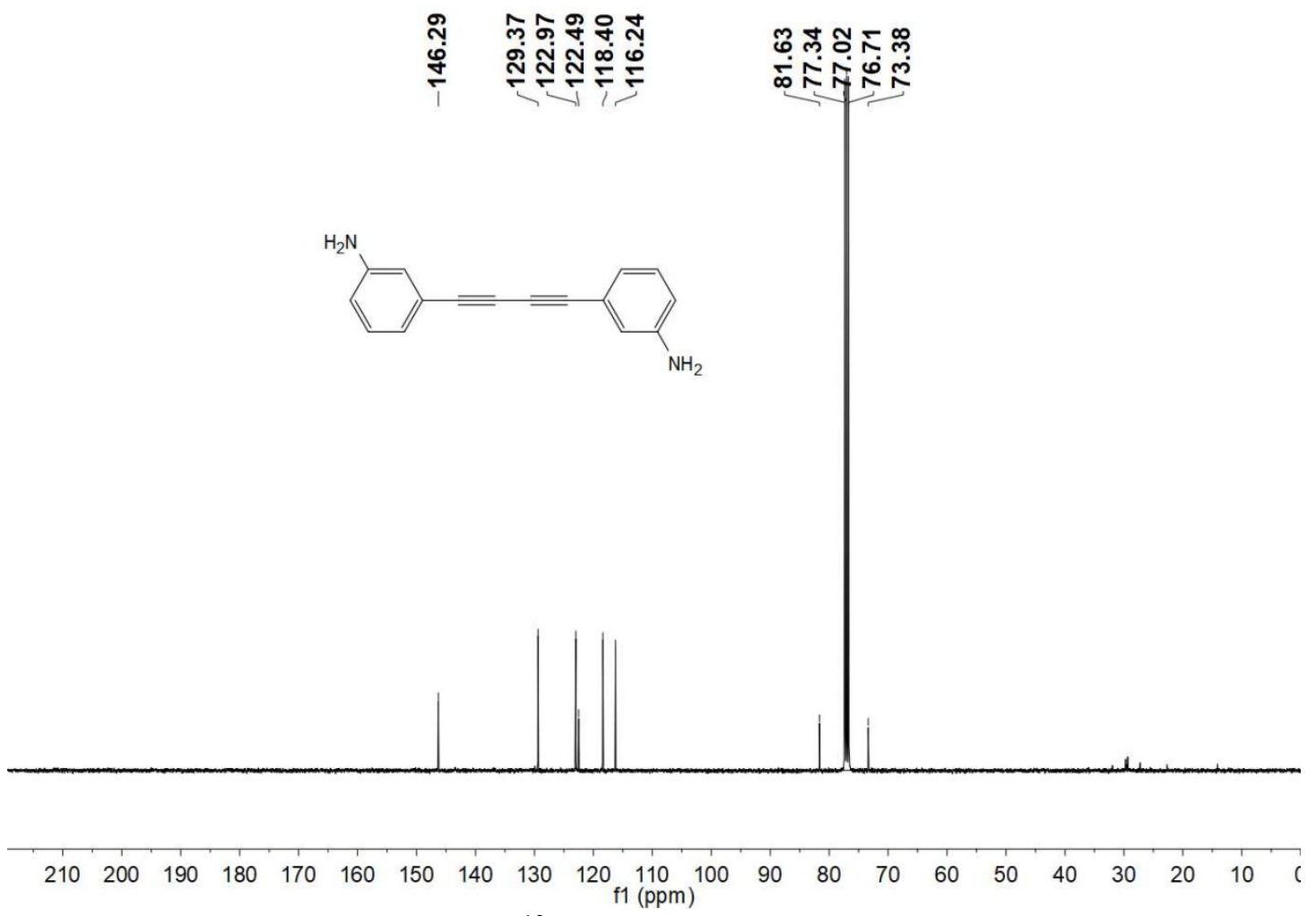

Figure S38. ${ }^{13} \mathrm{C}$ NMR spectroscopy of $\mathbf{3 m}$. 


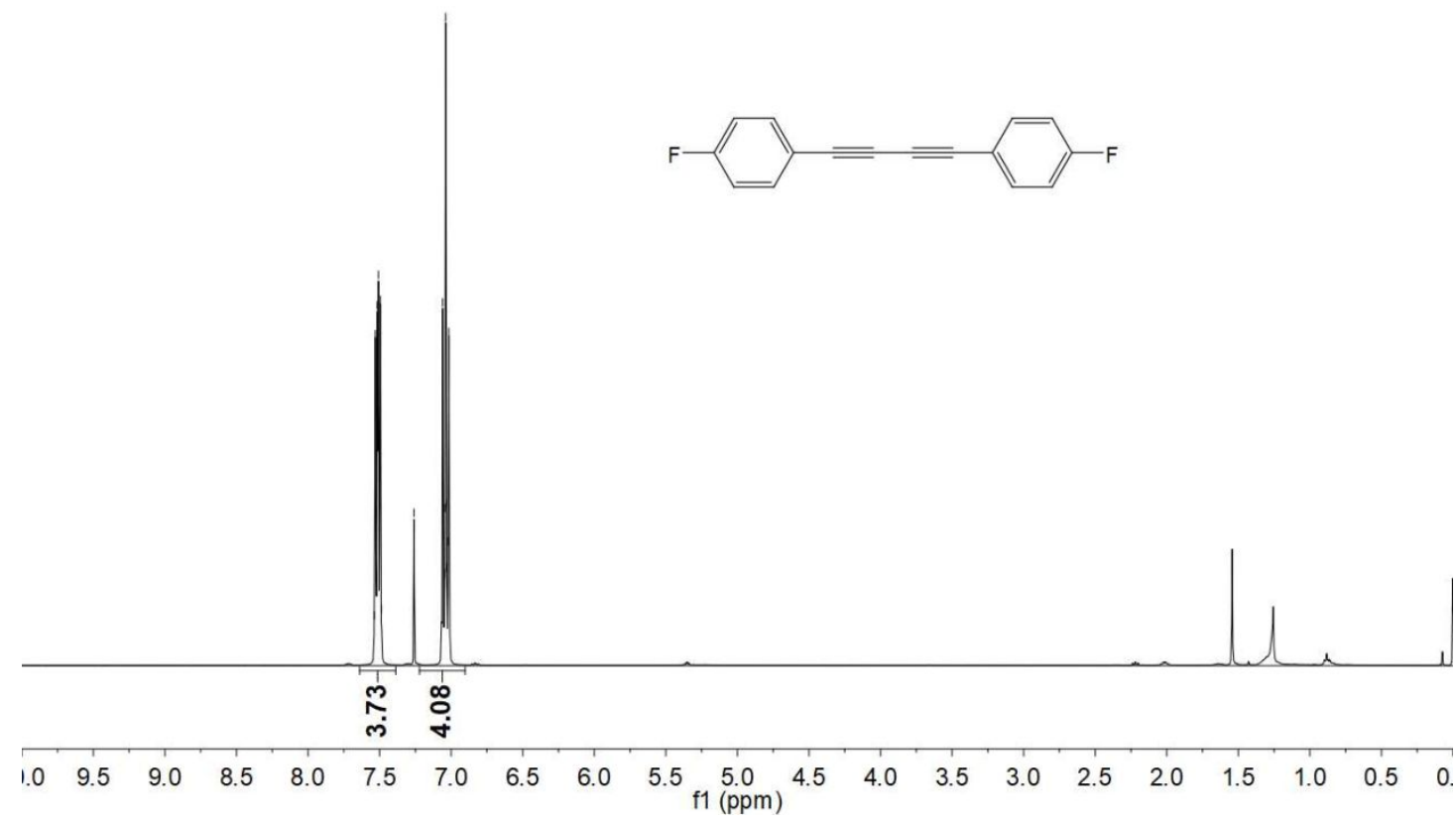

Figure S39. ${ }^{1} \mathrm{H}$ NMR spectroscopy of $\mathbf{3 n}$.

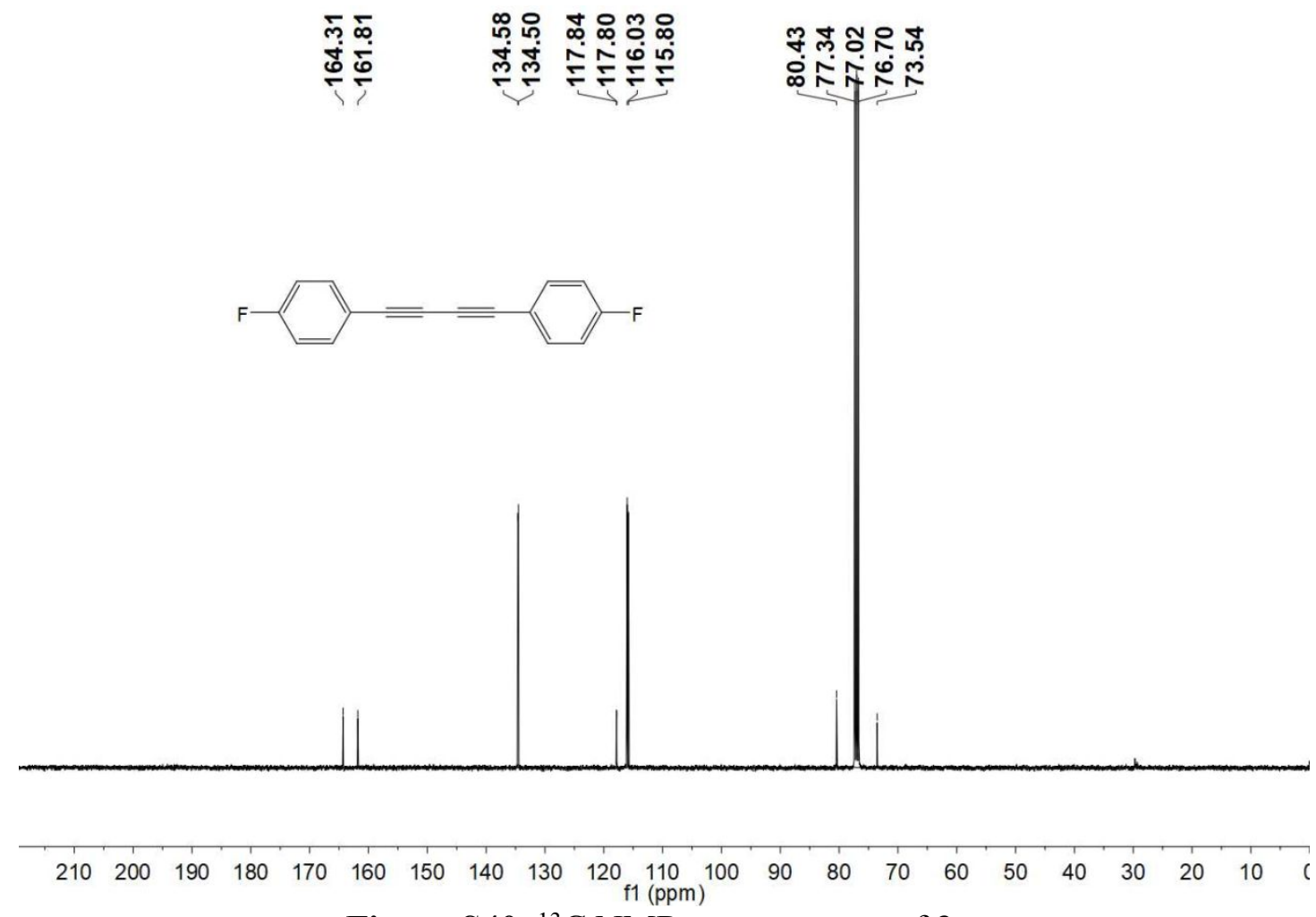

Figure S40. ${ }^{13} \mathrm{C}$ NMR spectroscopy of $\mathbf{3 n}$. 

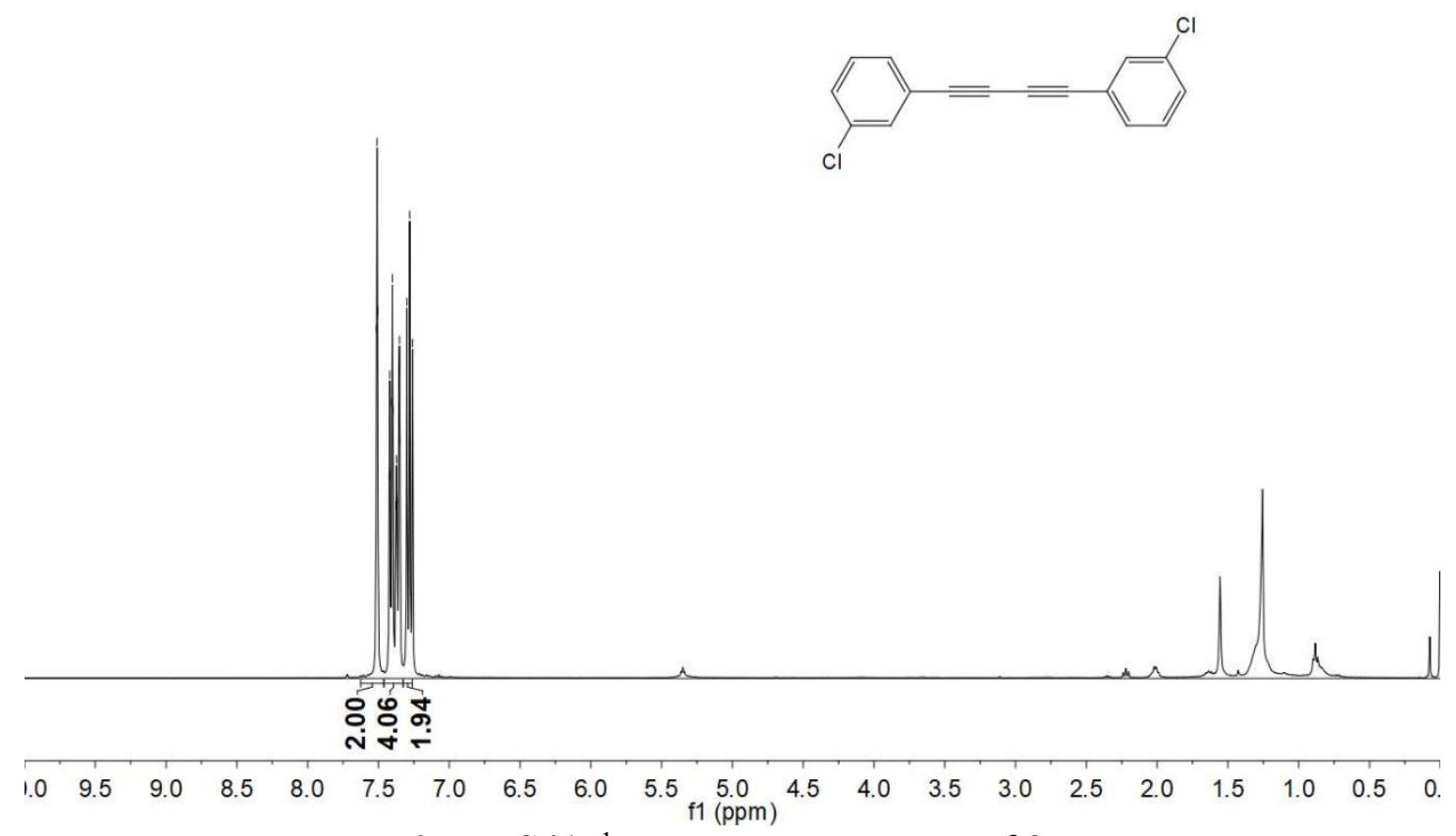

Figure S41. ${ }^{1} \mathrm{H}$ NMR spectroscopy of $\mathbf{3 o}$.

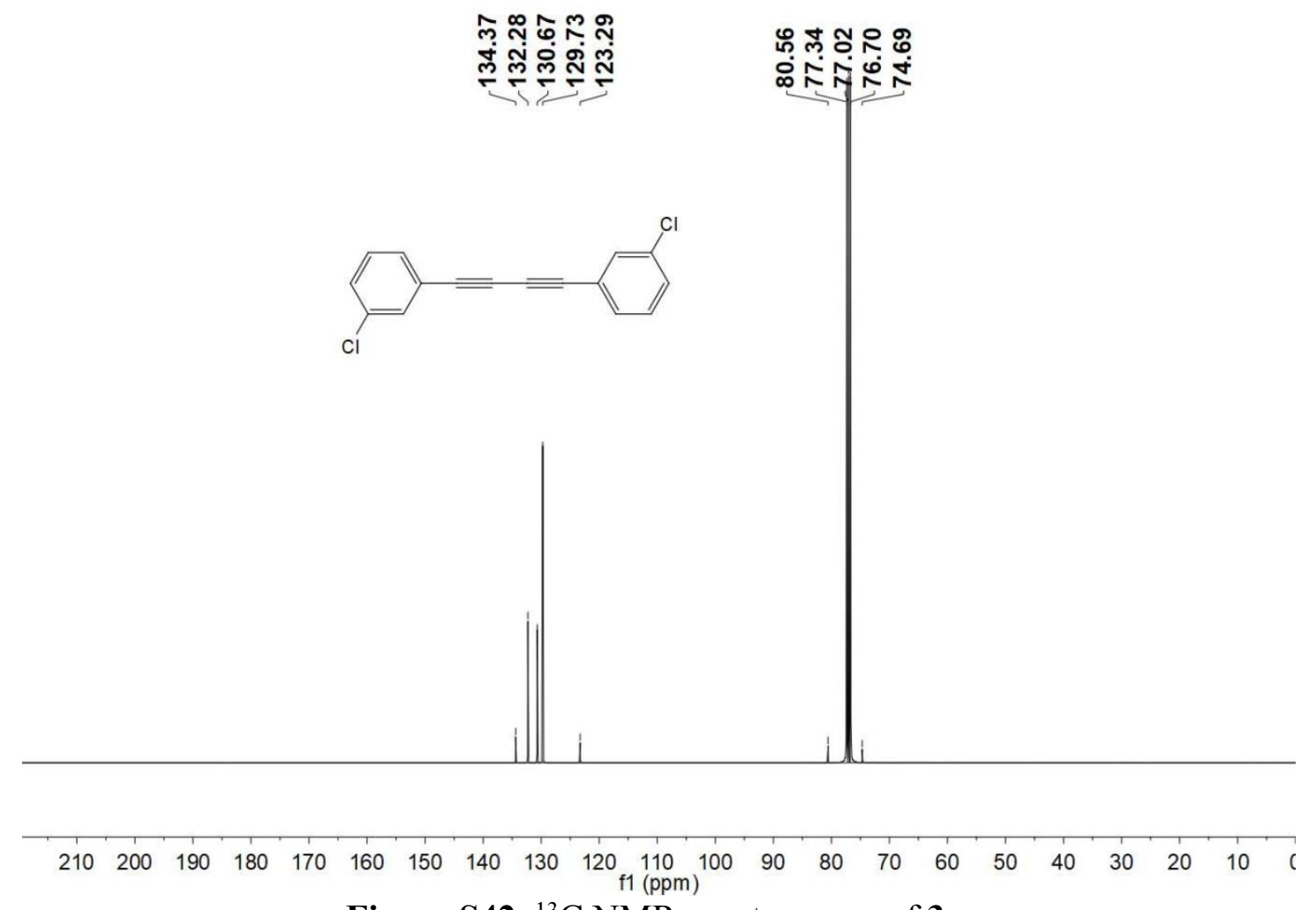

Figure S42. ${ }^{13} \mathrm{C}$ NMR spectroscopy of 30 . 


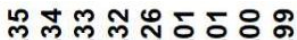

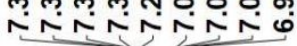
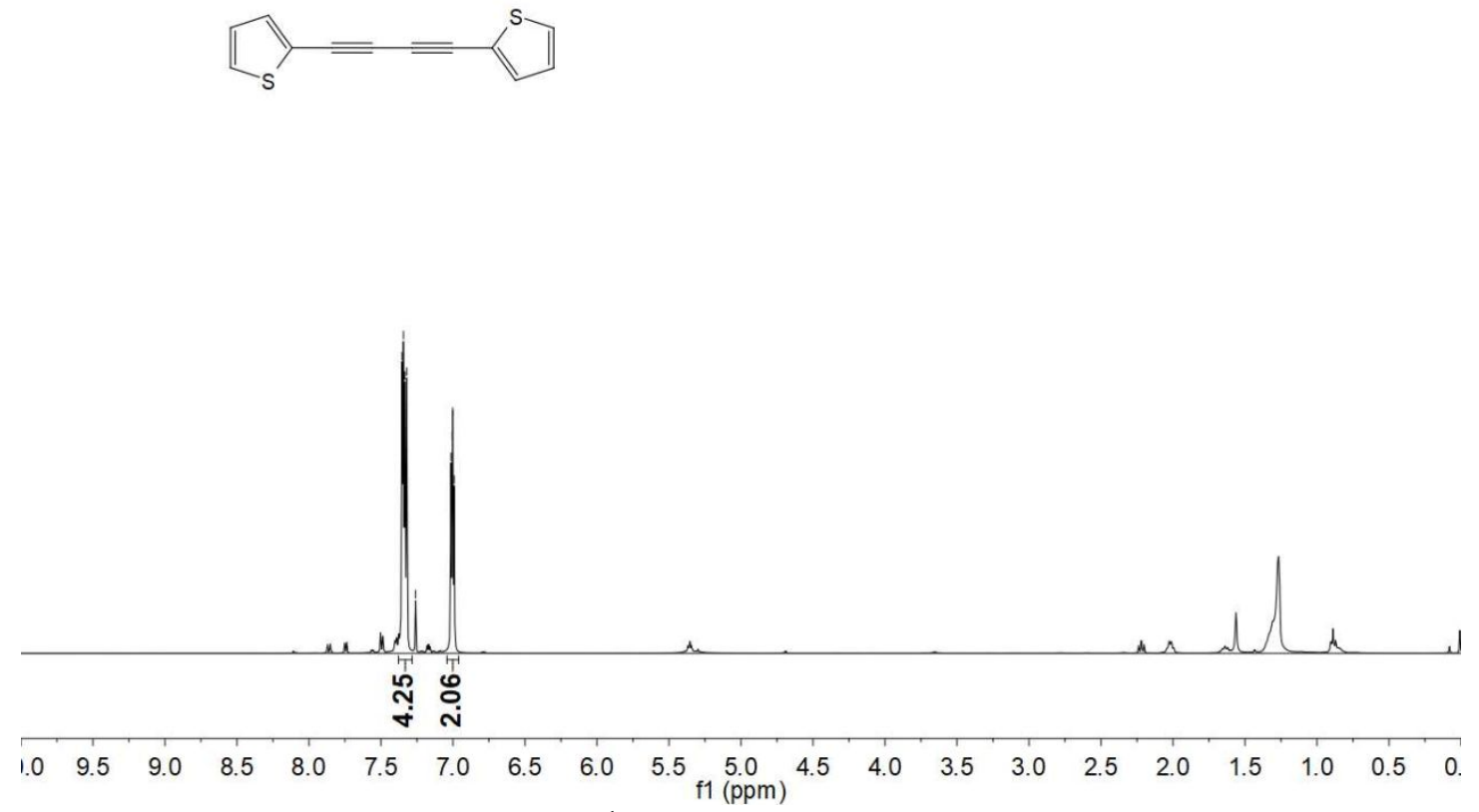

Figure S43. ${ }^{1} \mathrm{H}$ NMR spectroscopy of $\mathbf{3 p}$.

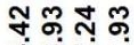

产 ָ̊ำ

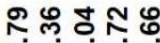

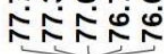
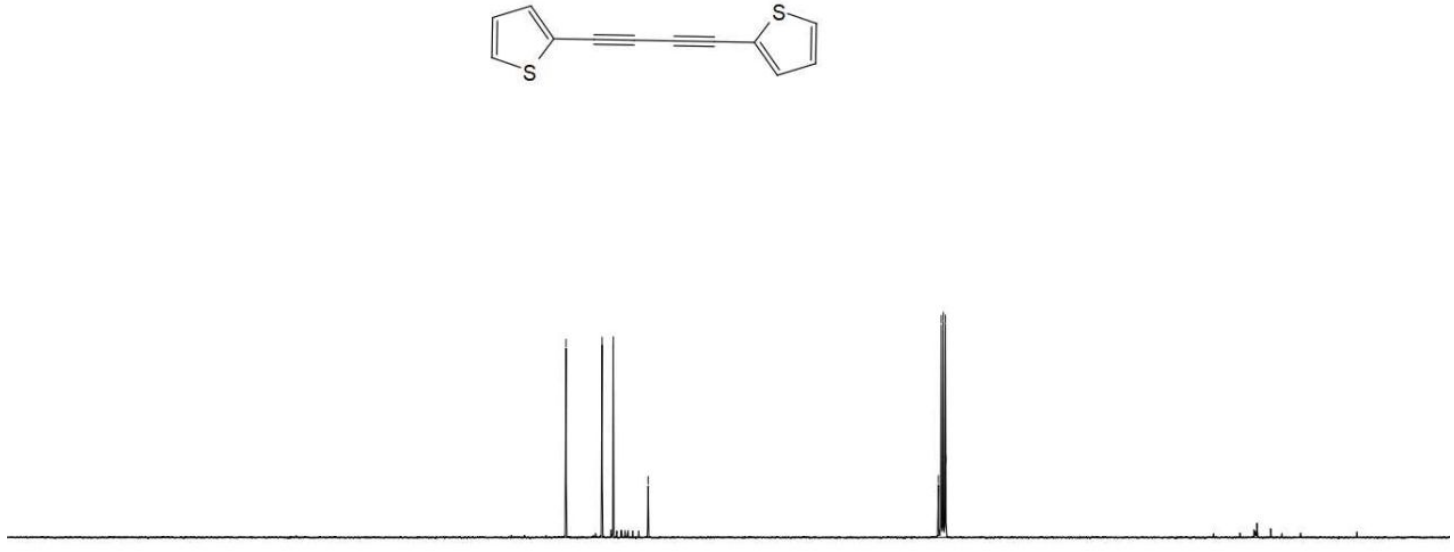

$\begin{array}{lllllllllllllllllllll}210 & 200 & 190 & 180 & 170 & 160 & 150 & 140 & 130 & 120 & 110 & 100 & 90 & 80 & 70 & 60 & 50 & 40 & 30 & 20 & 10\end{array}$

Figure S44. ${ }^{13} \mathrm{C}$ NMR spectroscopy of $3 p$. 
冓
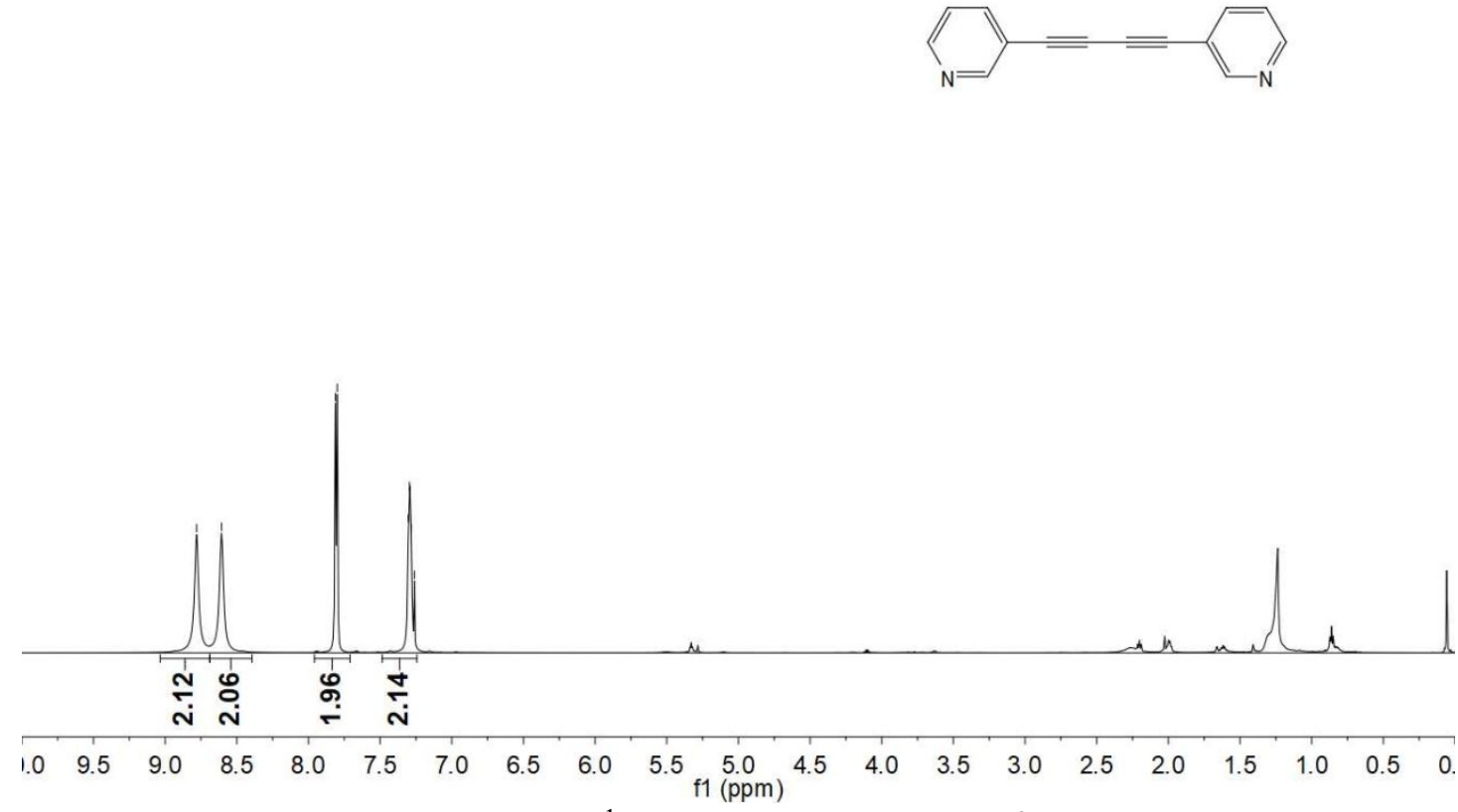

Figure S45. ${ }^{1} \mathrm{H}$ NMR spectroscopy of $\mathbf{3 q}$.

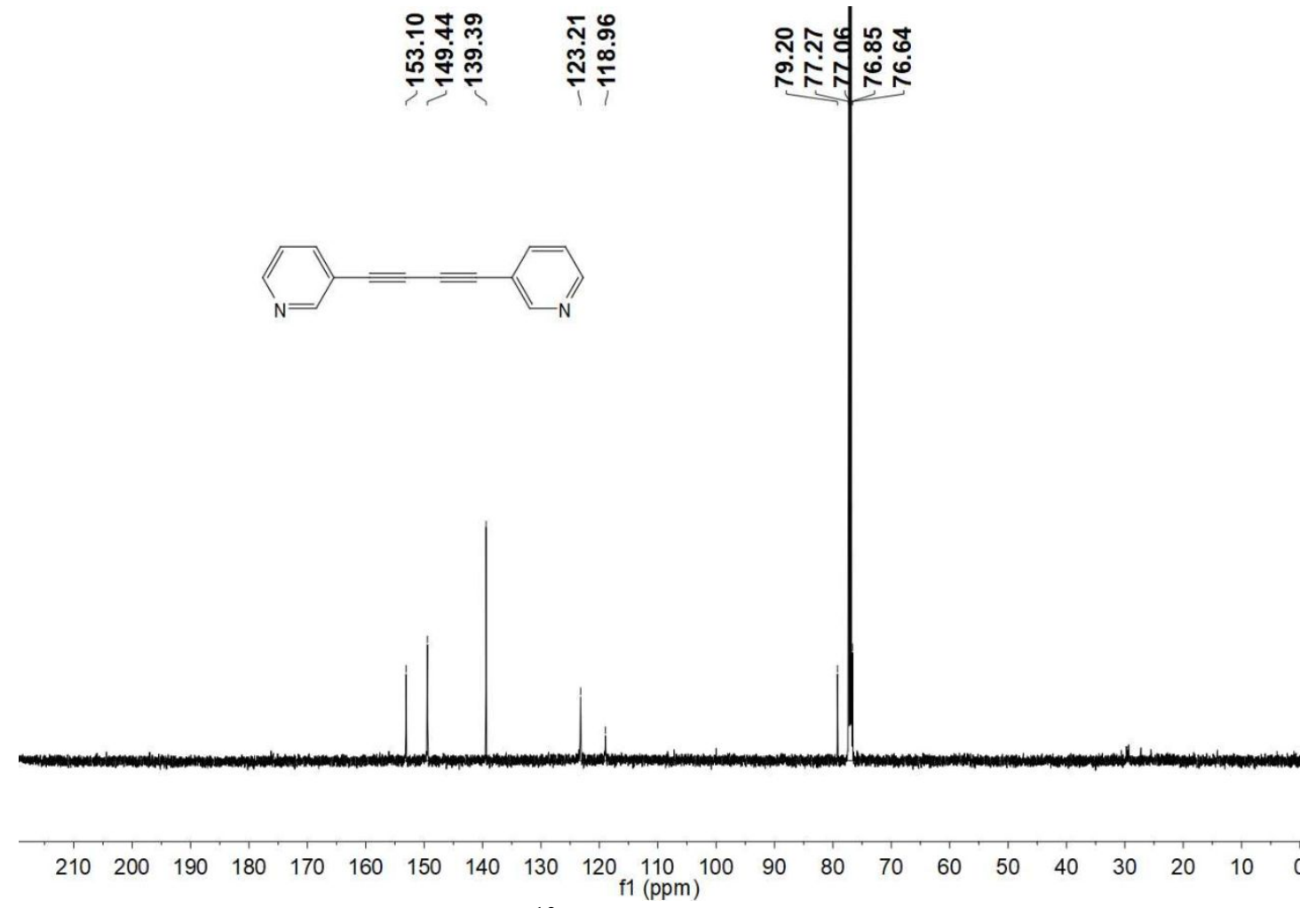

Figure S46. ${ }^{13} \mathrm{C}$ NMR spectroscopy of $\mathbf{3 q}$. 


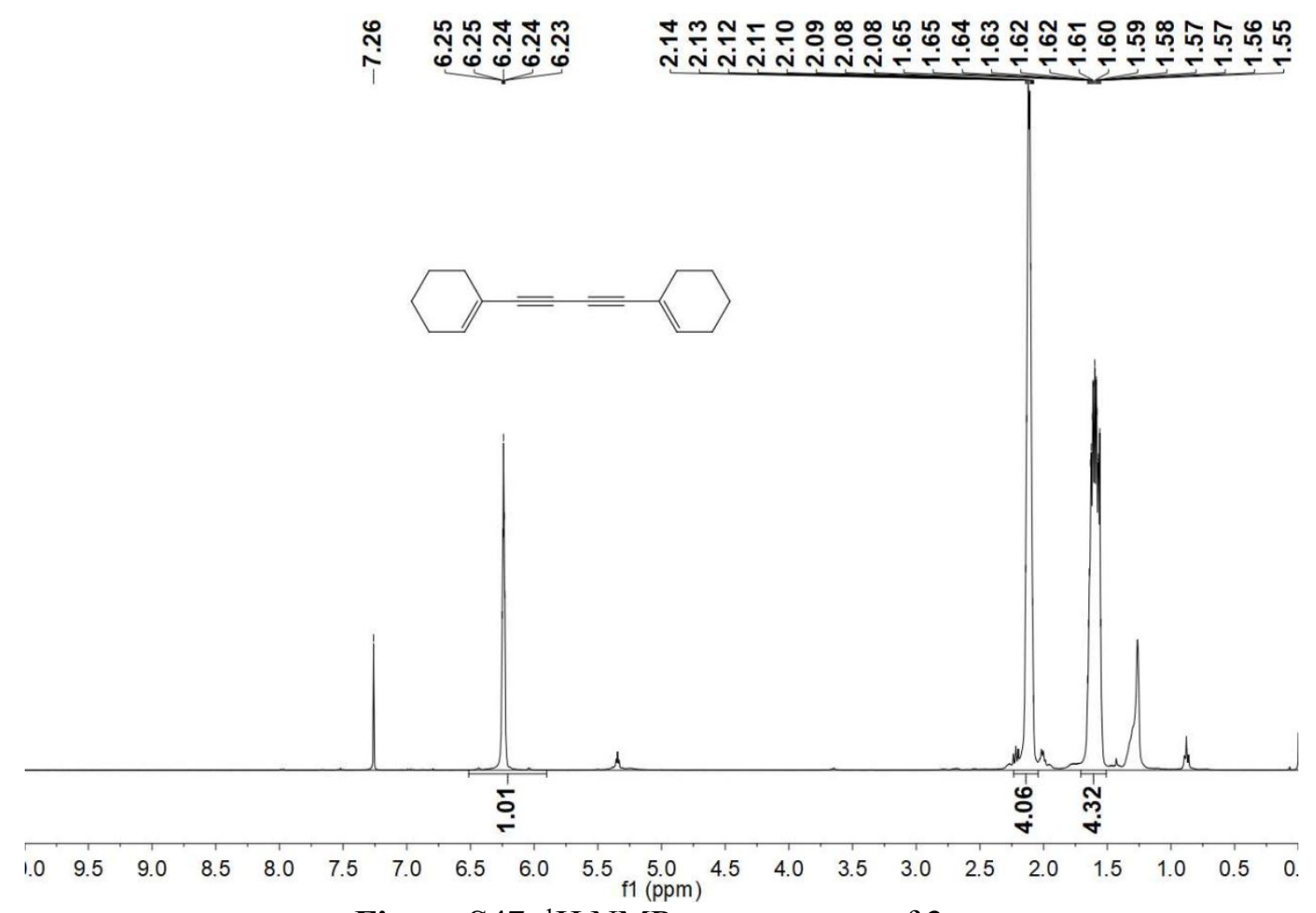

Figure S47. ${ }^{1} \mathrm{H}$ NMR spectroscopy of $3 \mathbf{r}$.

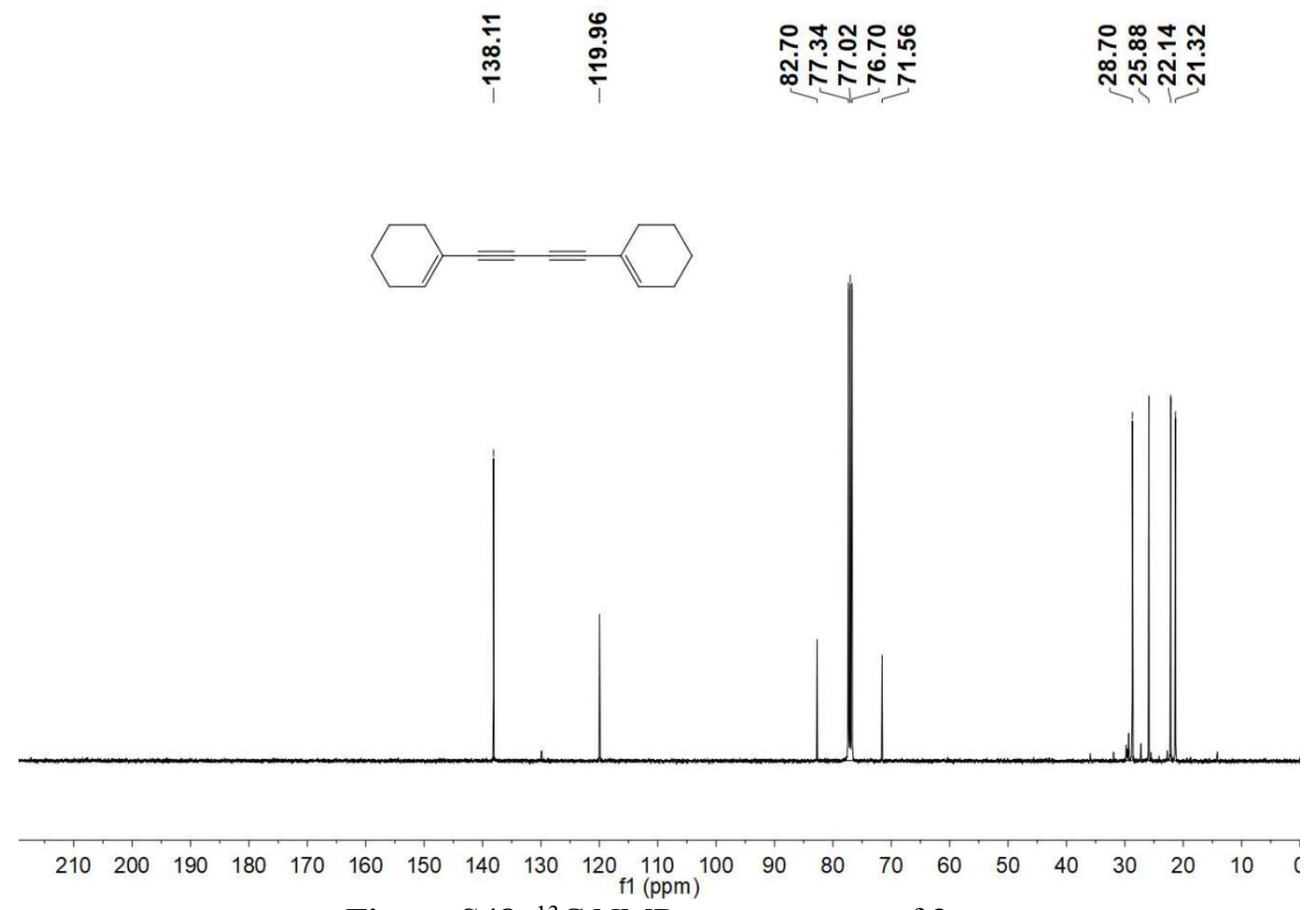

Figure S48. ${ }^{13} \mathrm{C}$ NMR spectroscopy of $\mathbf{3 r}$. 


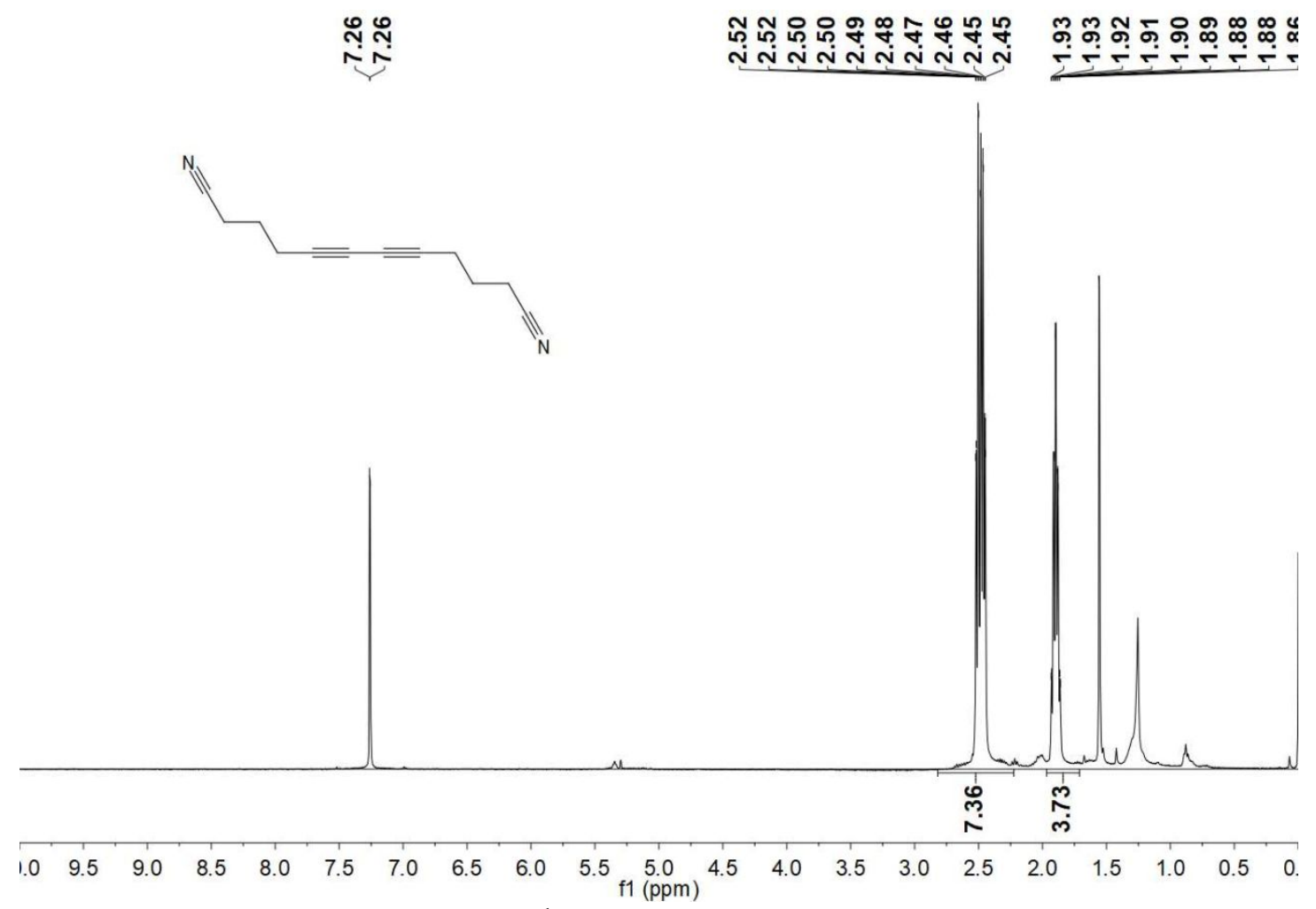

Figure S49. ${ }^{1} \mathrm{H}$ NMR spectroscopy of $\mathbf{3 s}$.

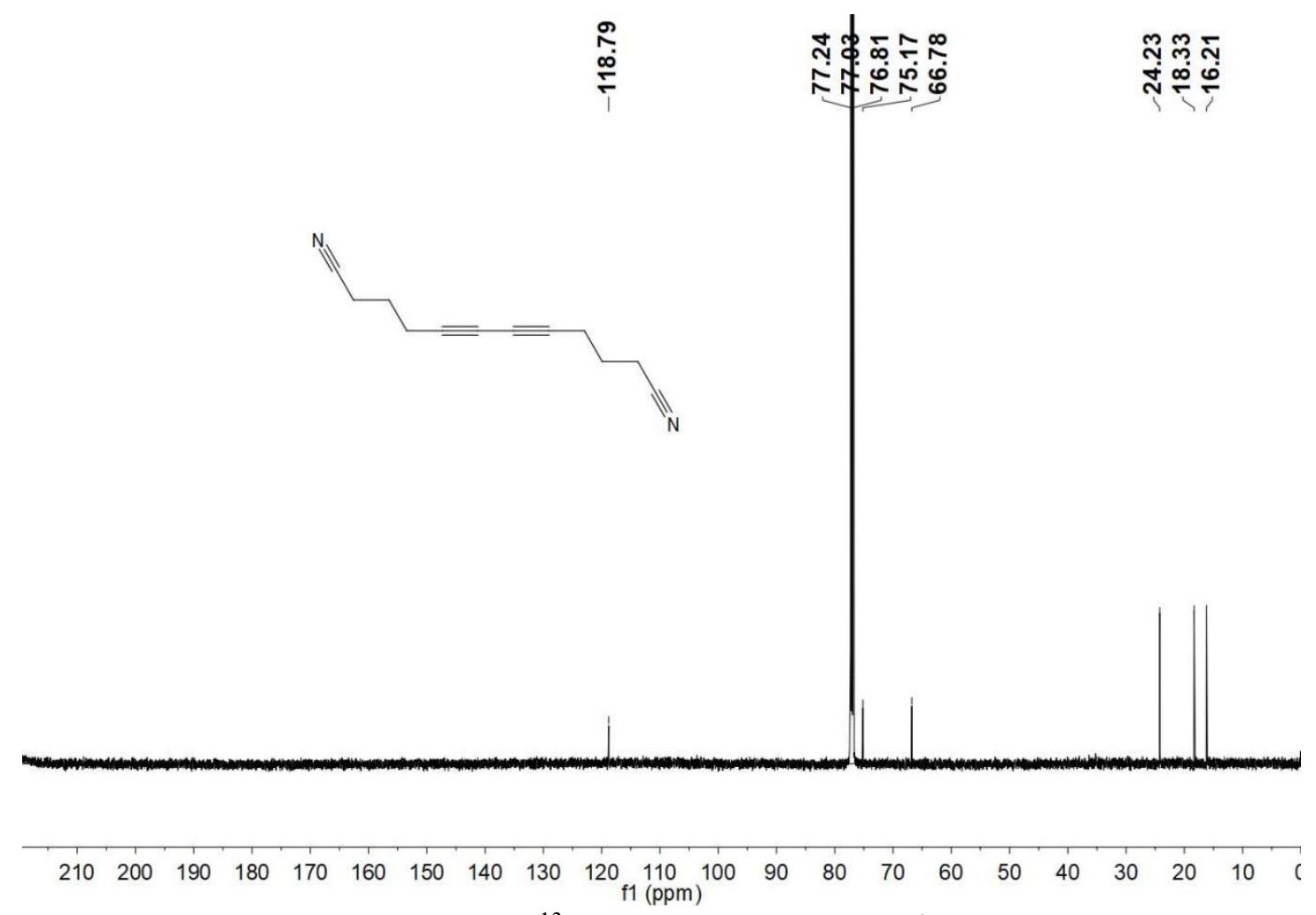

Figure S50. ${ }^{13} \mathrm{C}$ NMR spectroscopy of $\mathbf{3 s}$. 


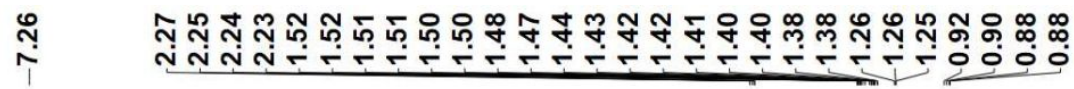

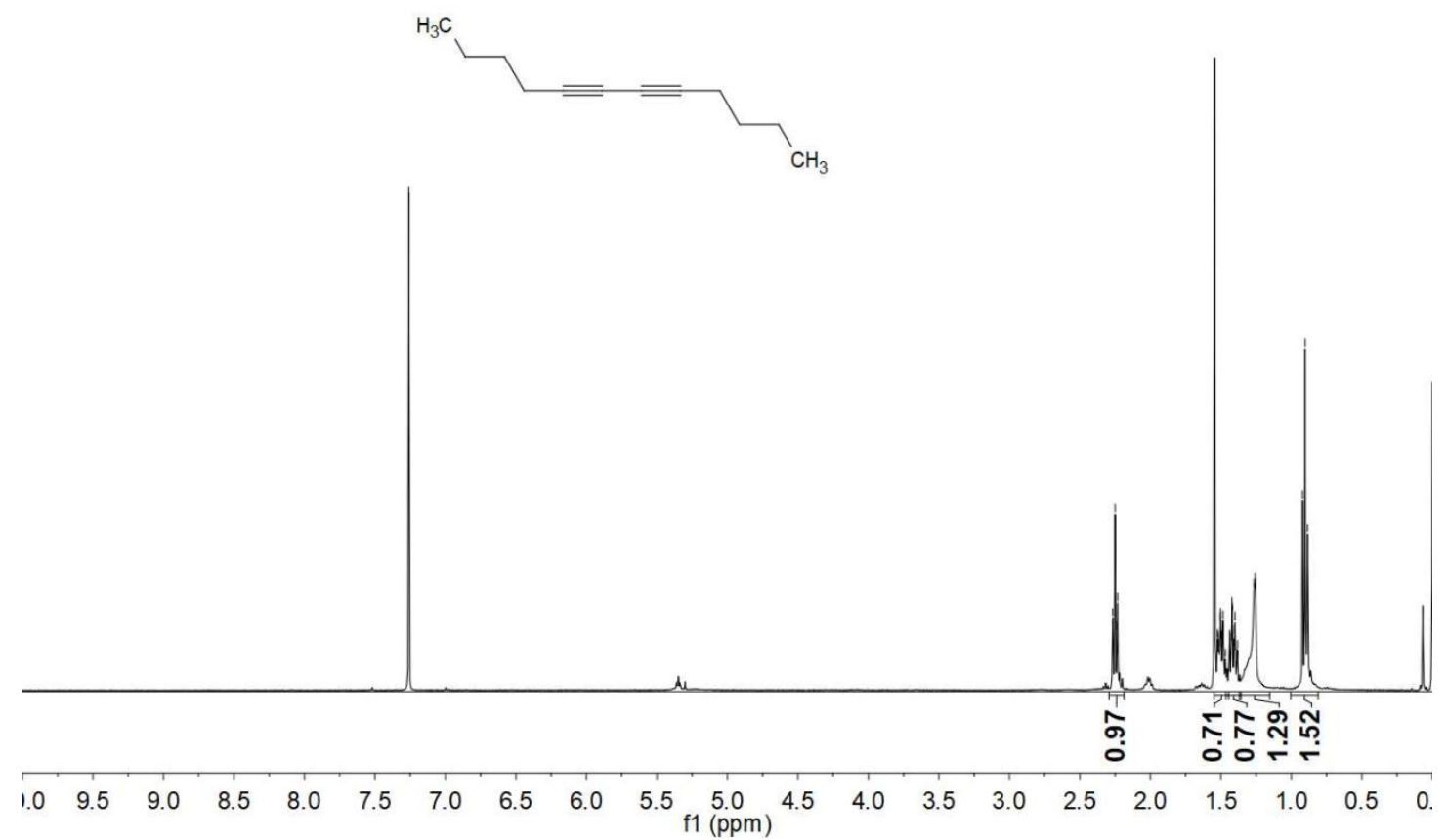

Figure S51. ${ }^{1} \mathrm{H}$ NMR spectroscopy of $3 \mathbf{t}$.

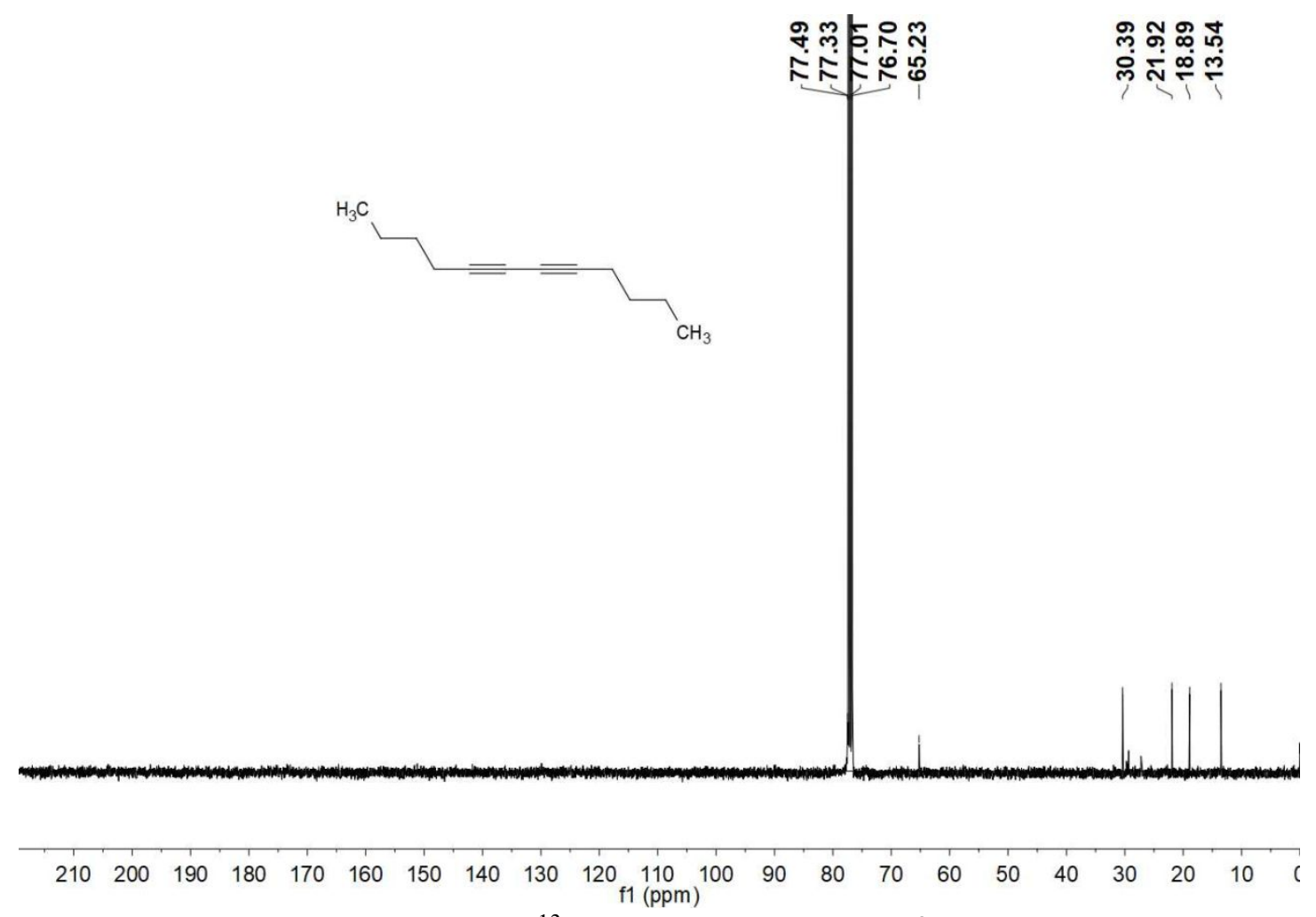

Figure S52. ${ }^{13} \mathrm{C}$ NMR spectroscopy of $3 \mathrm{t}$. 


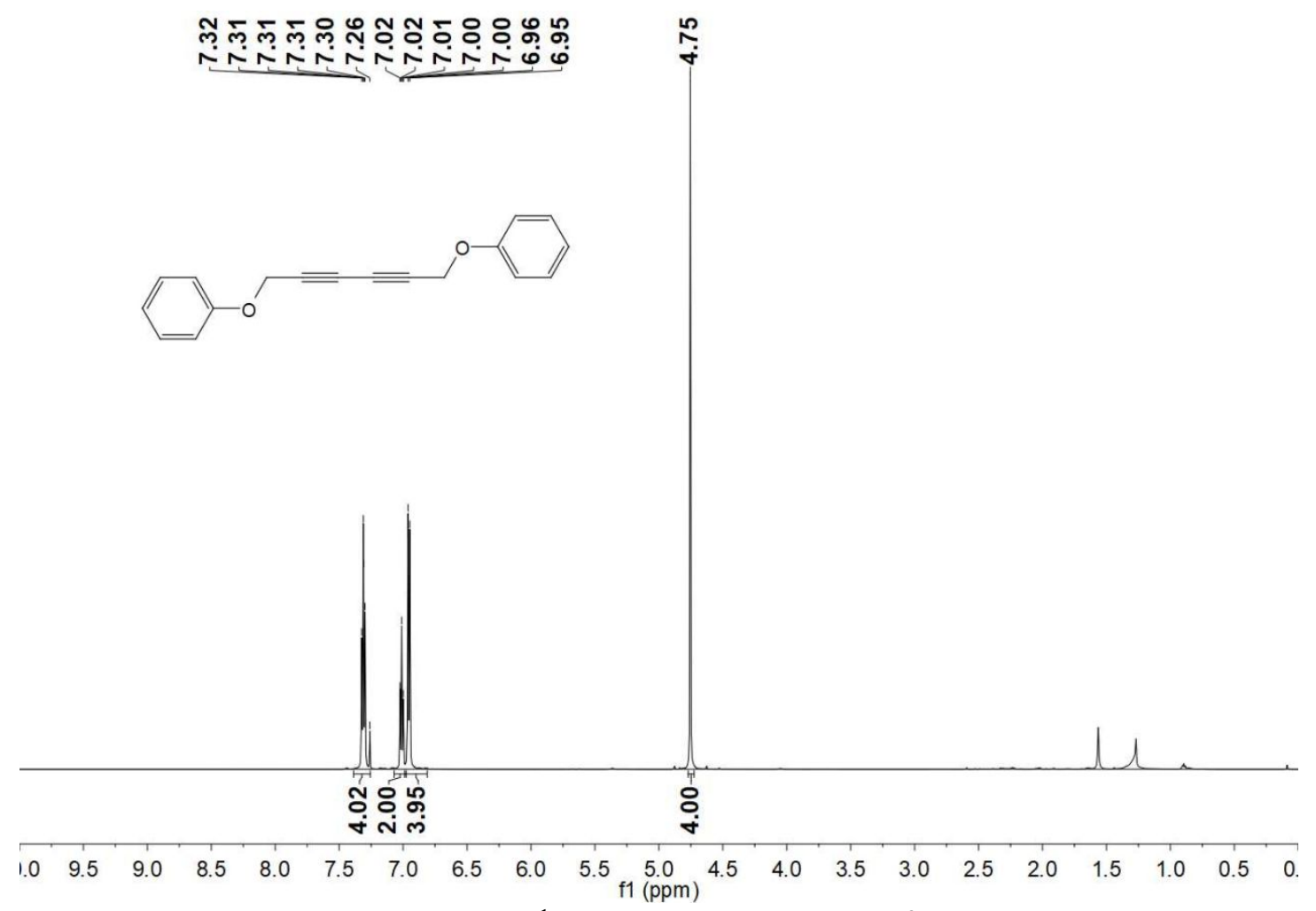

Figure S53. ${ }^{1} \mathrm{H}$ NMR spectroscopy of $\mathbf{3 u}$.

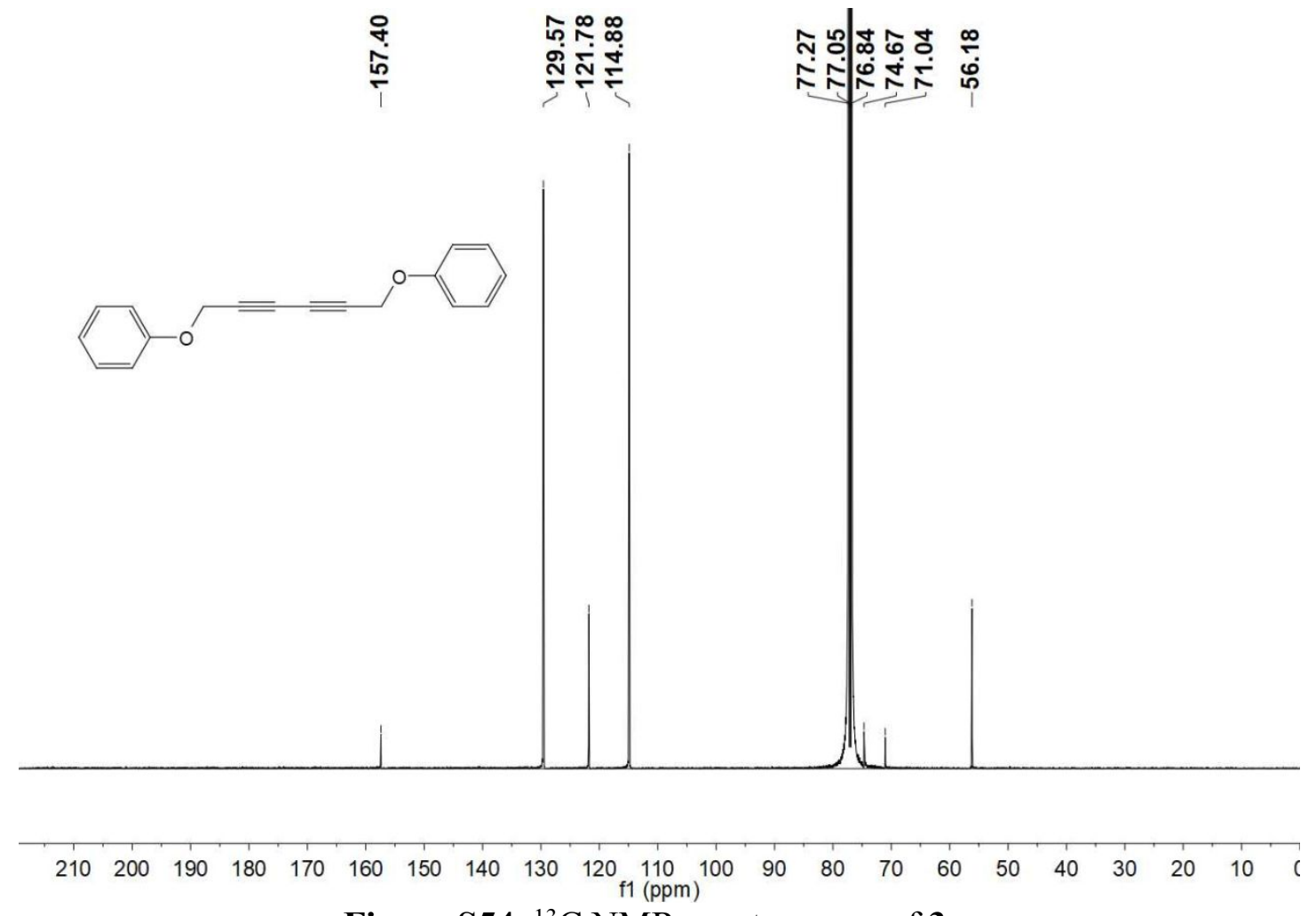

Figure S54. ${ }^{13} \mathrm{C}$ NMR spectroscopy of $3 \mathbf{u}$. 

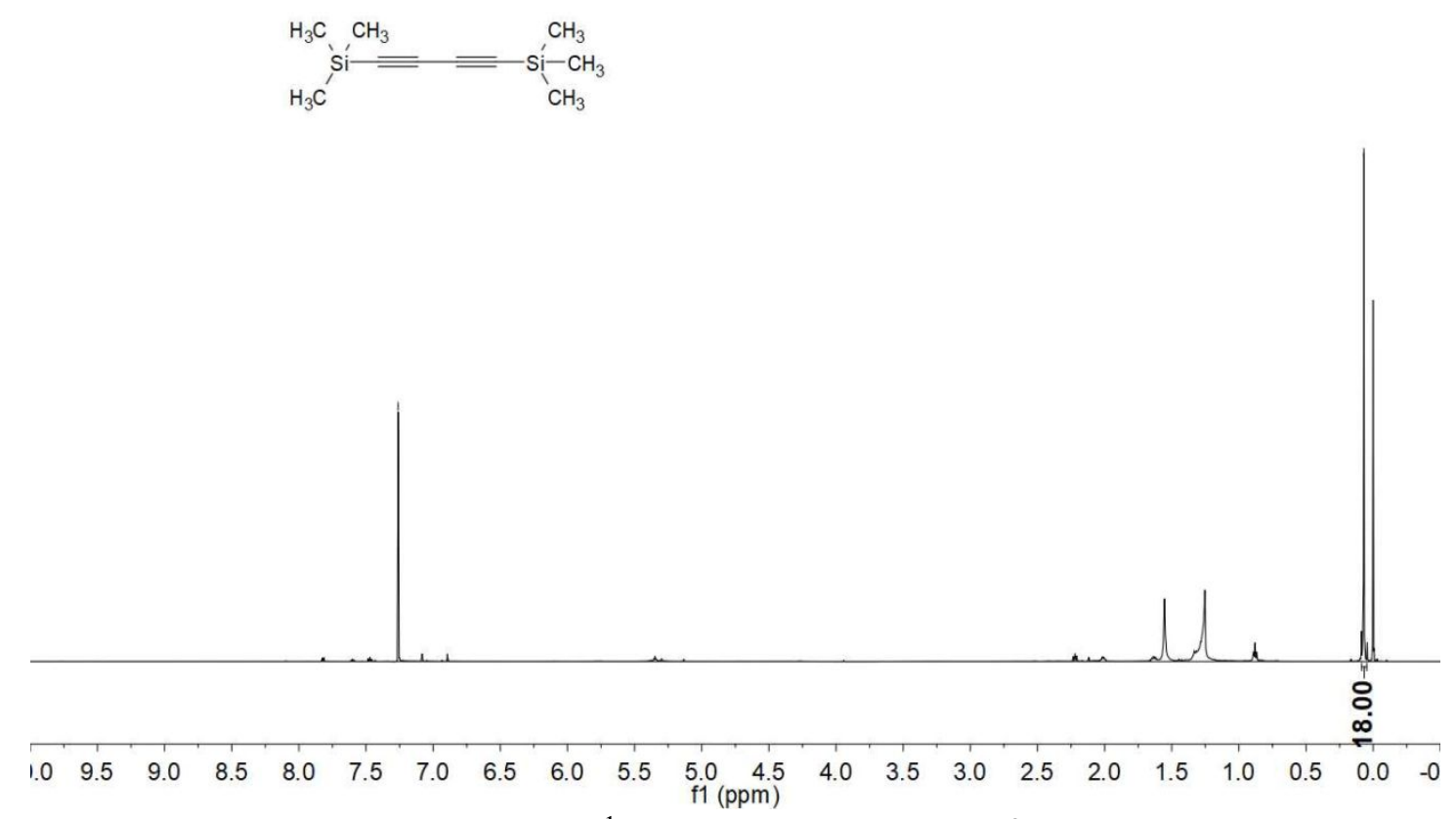

Figure S55. ${ }^{1} \mathrm{H}$ NMR spectroscopy of $\mathbf{3 v}$.

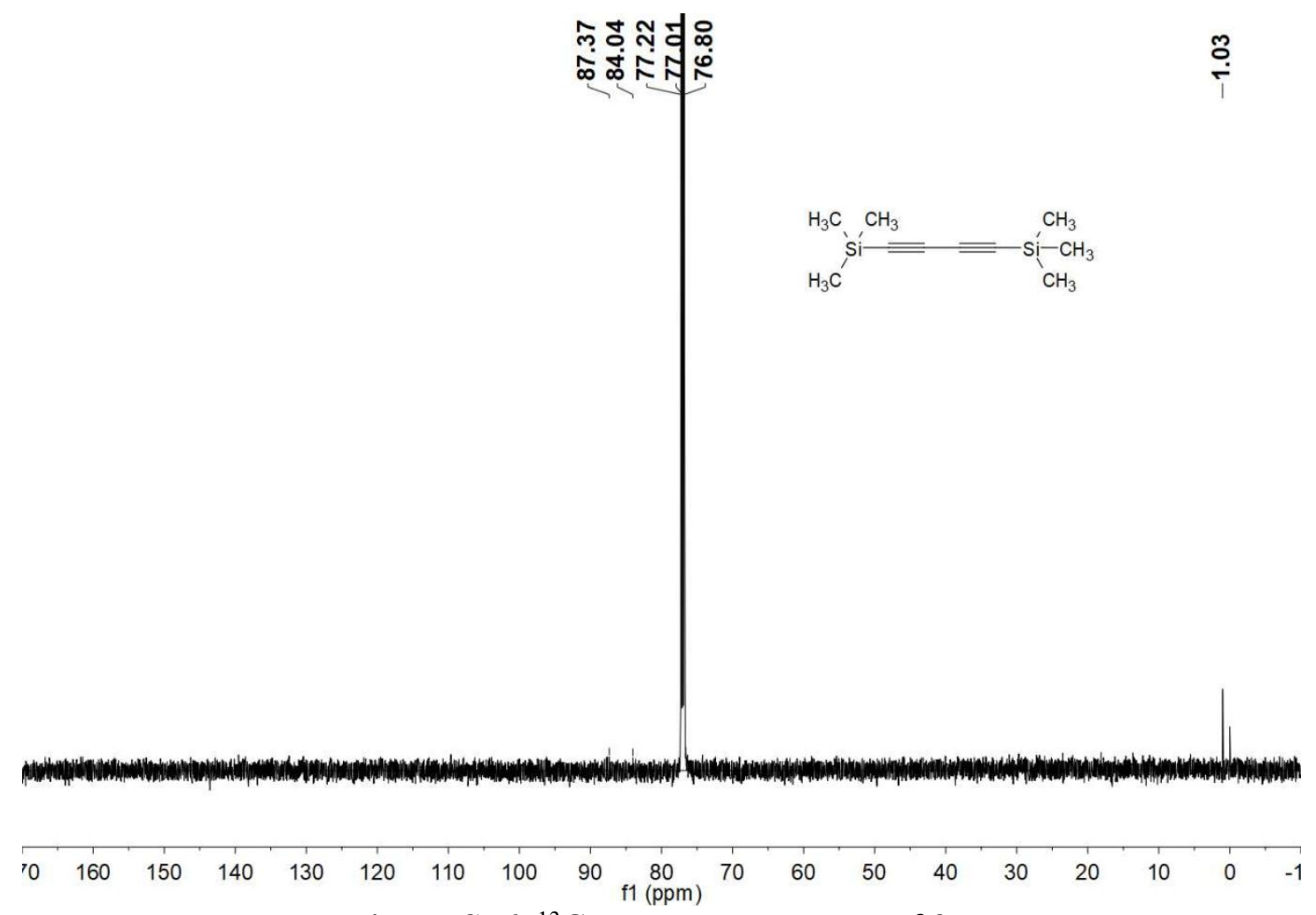

Figure S56. ${ }^{13} \mathrm{C}$ NMR spectroscopy of $\mathbf{3 v}$. 

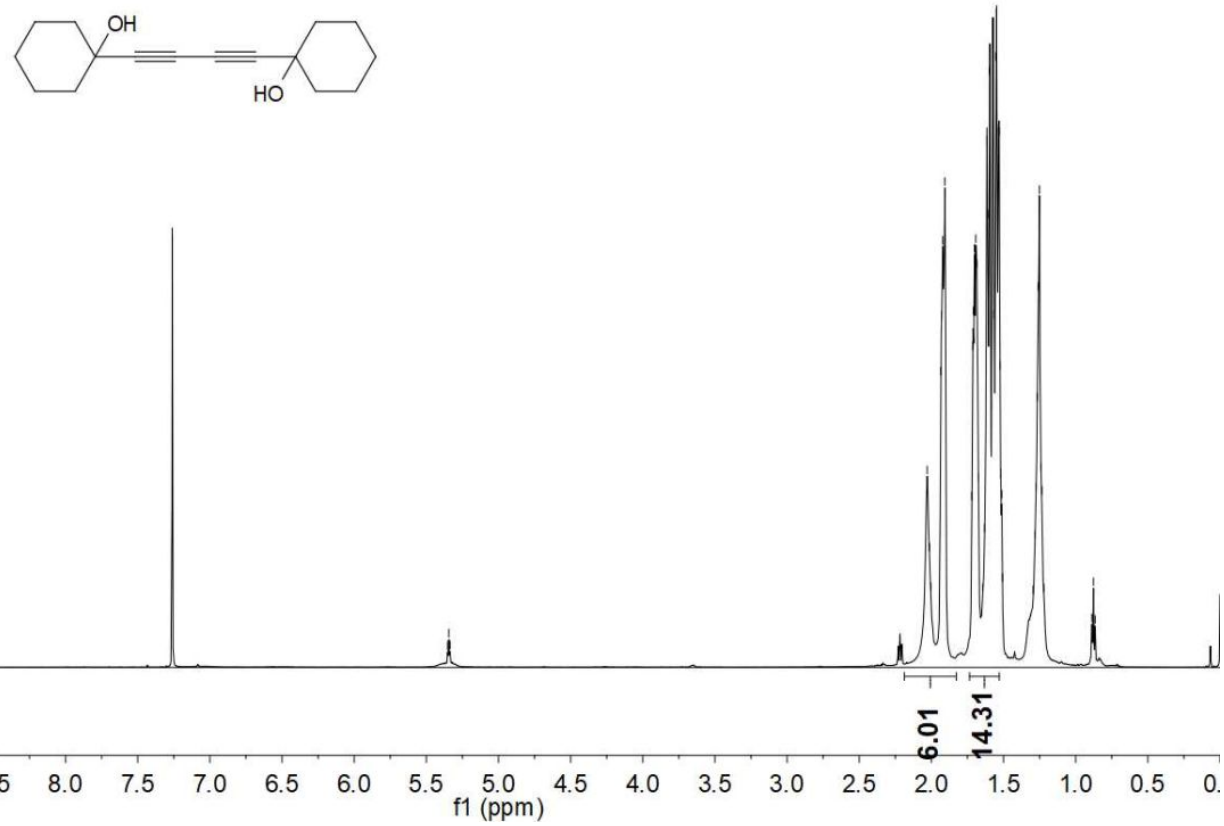

Figure S57. ${ }^{1} \mathrm{H}$ NMR spectroscopy of $\mathbf{3 w}$.

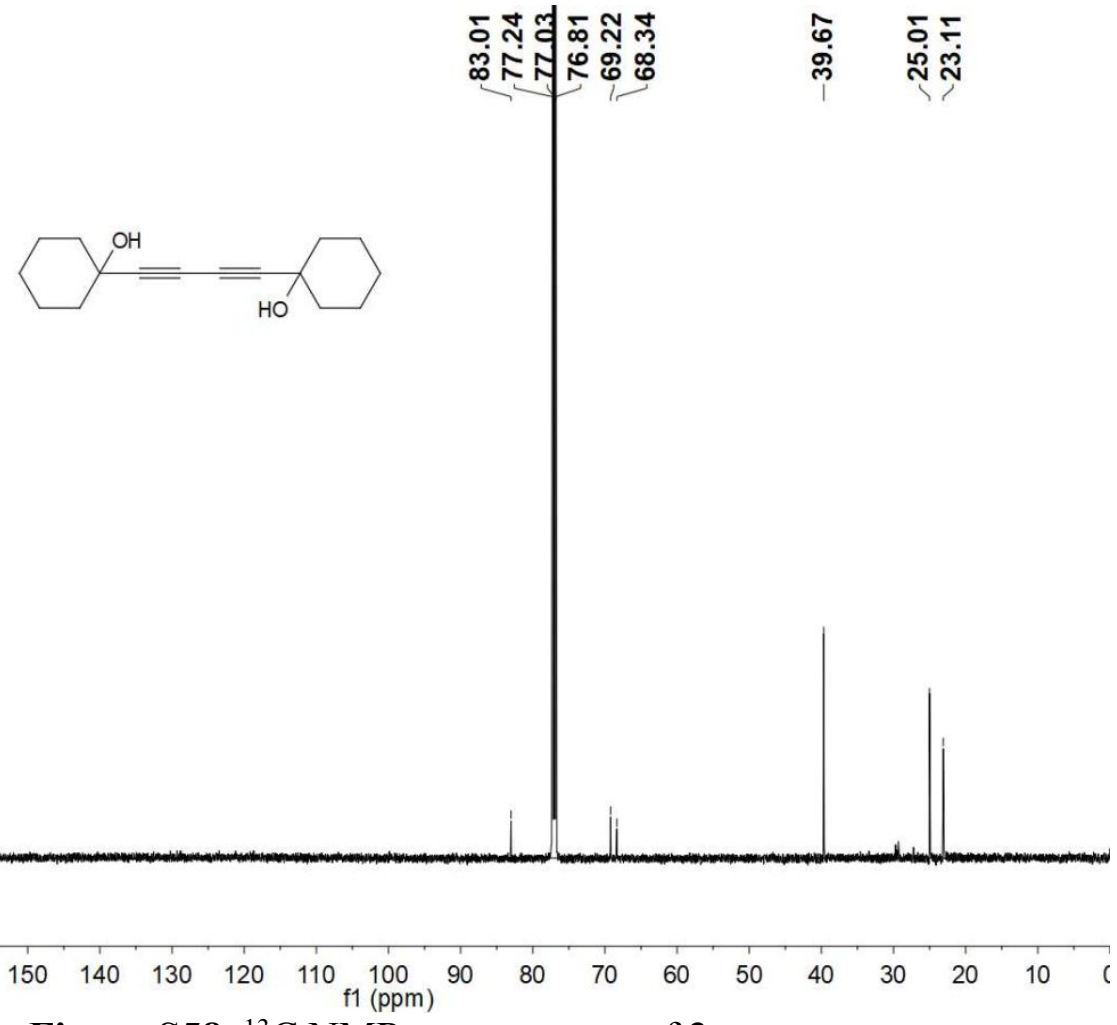

Figure S58. ${ }^{13} \mathrm{C}$ NMR spectroscopy of $\mathbf{3 w}$. 

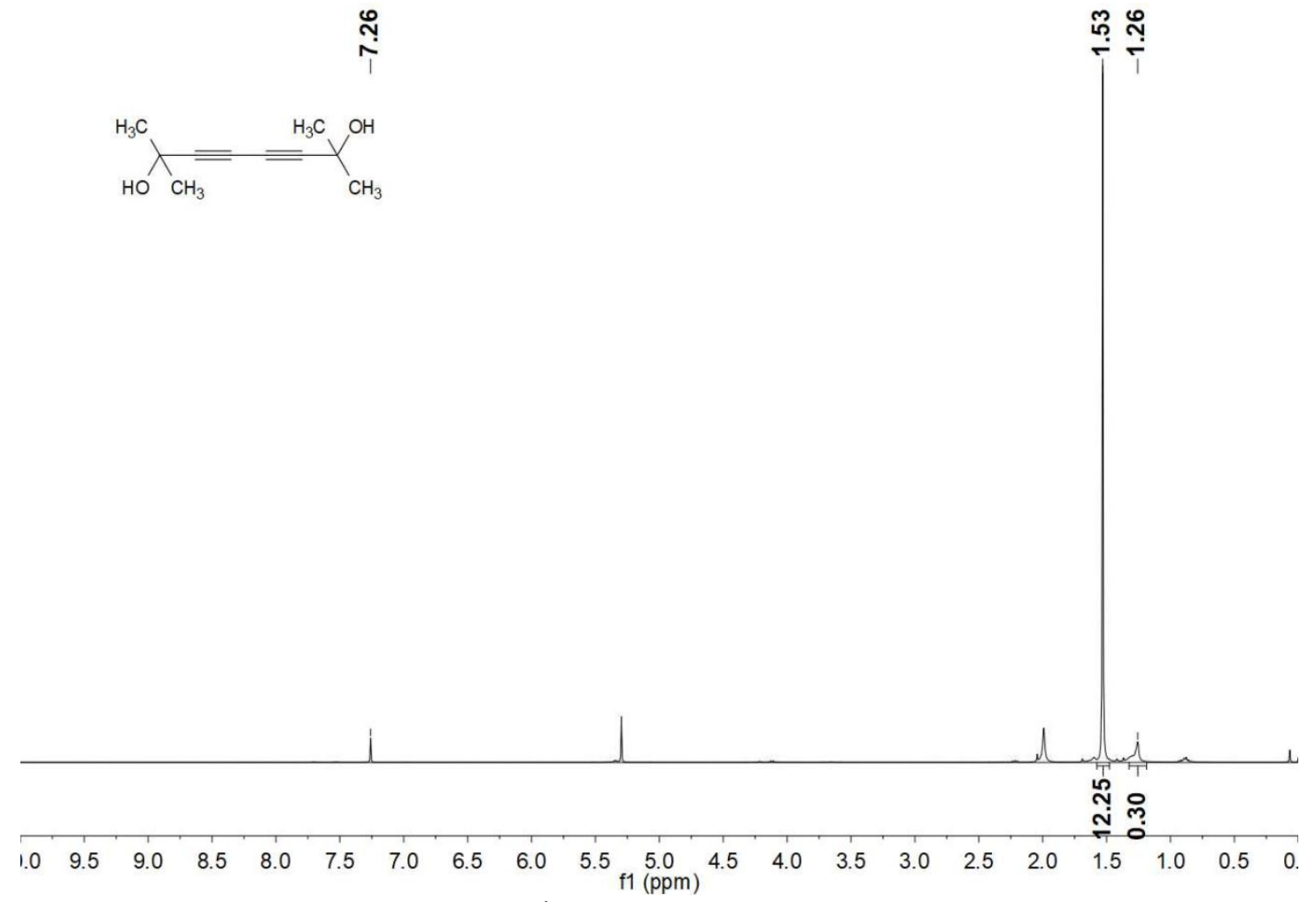

Figure S59. ${ }^{1} \mathrm{H}$ NMR spectroscopy of $\mathbf{3 x}$.

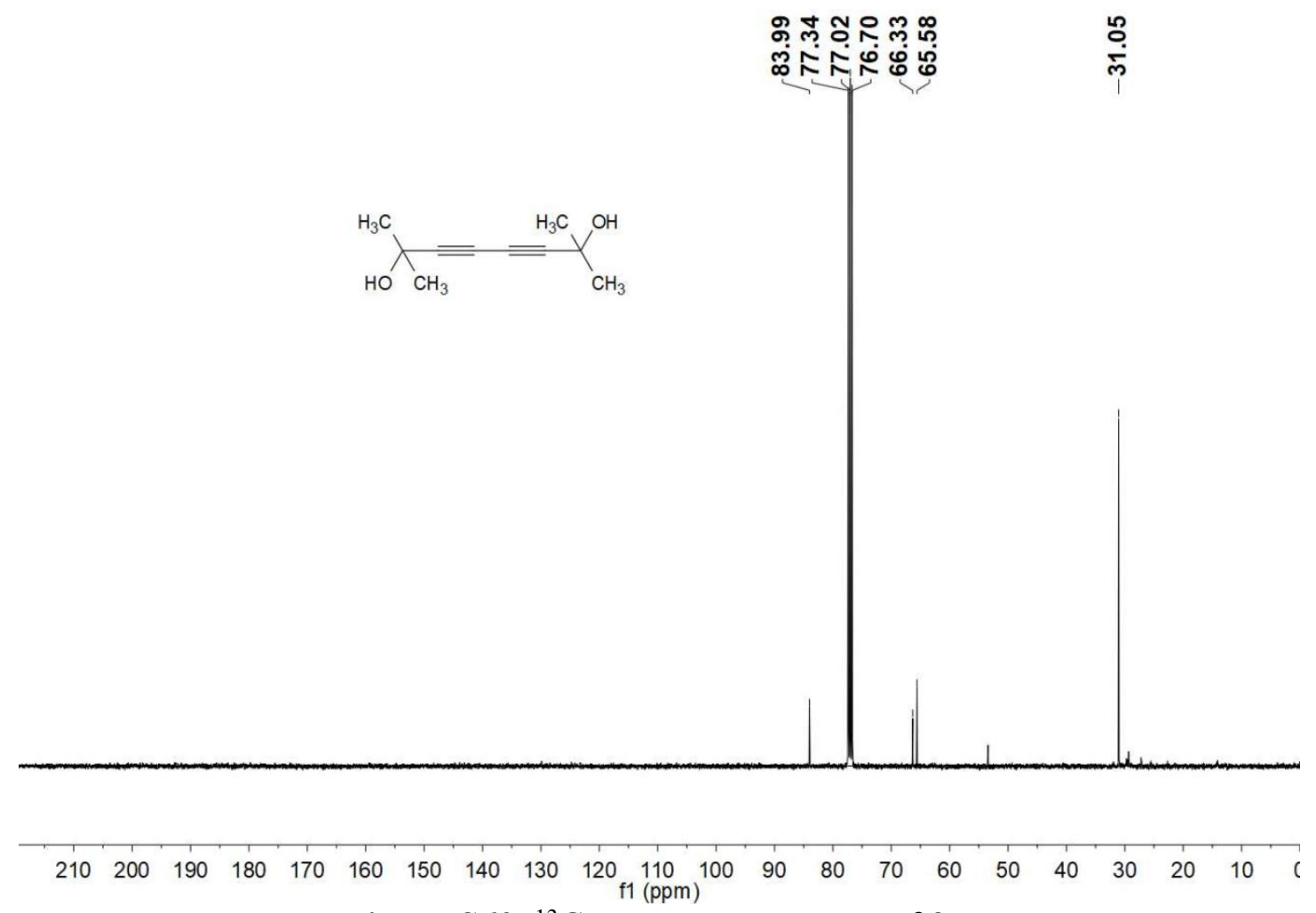

Figure S60. ${ }^{13} \mathrm{C}$ NMR spectroscopy of $\mathbf{3 x}$. 


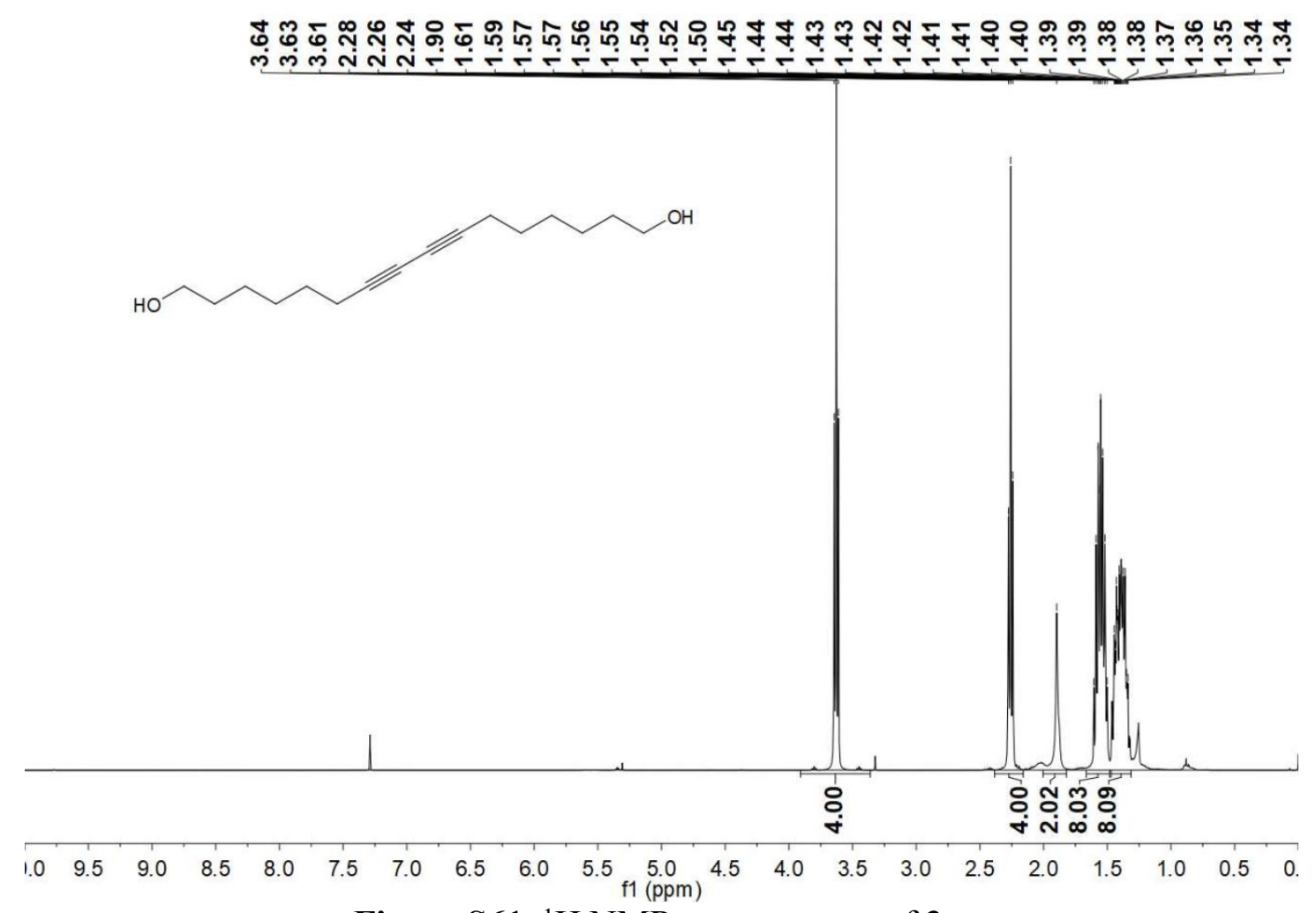

Figure S61. ${ }^{1} \mathrm{H}$ NMR spectroscopy of $\mathbf{3 y}$.

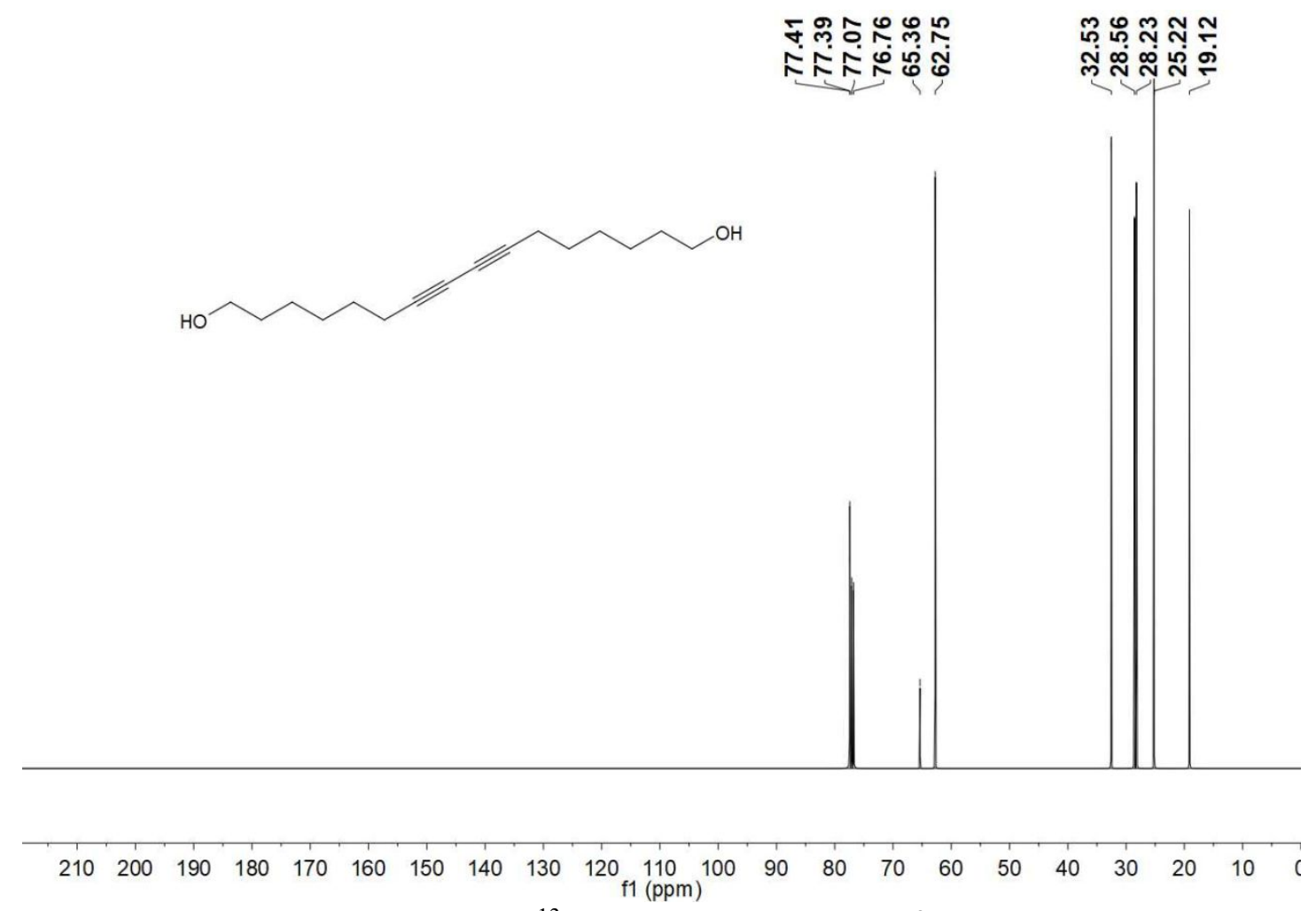

Figure S62. ${ }^{13} \mathrm{C}$ NMR spectroscopy of $\mathbf{3 y}$. 

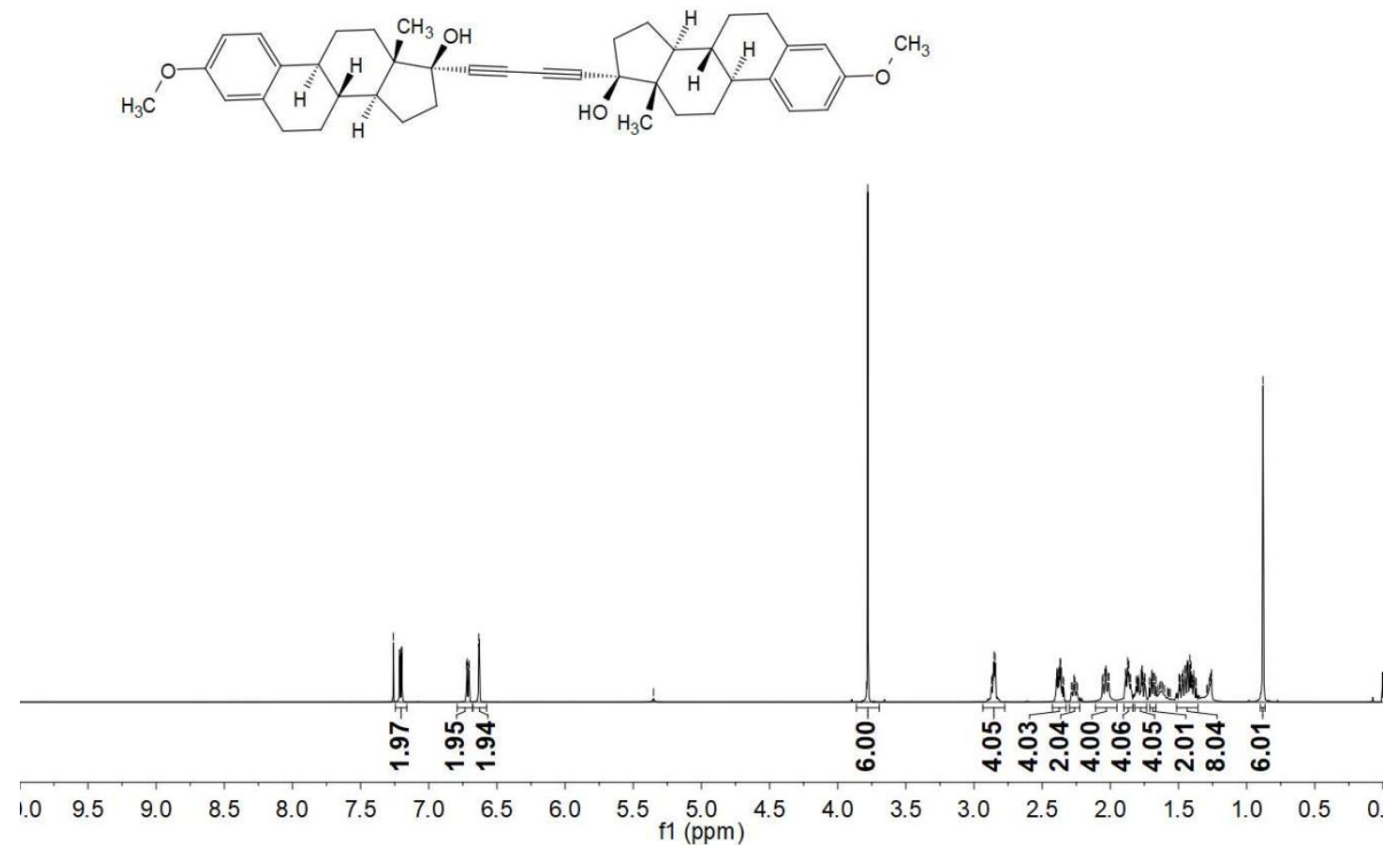

Figure S63. ${ }^{1} \mathrm{H}$ NMR spectroscopy of $\mathbf{3 z}$.

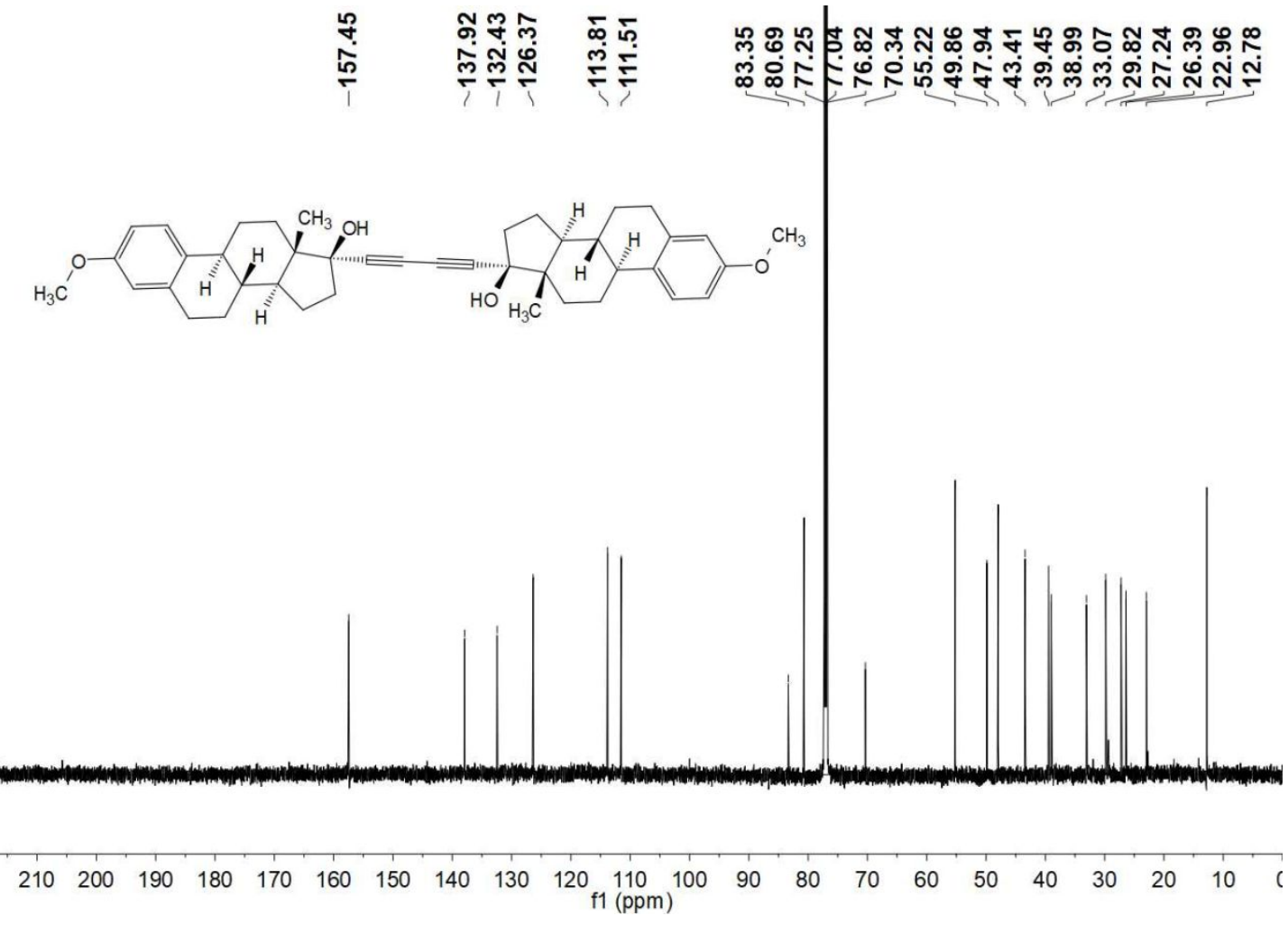

Figure S64. ${ }^{13} \mathrm{C}$ NMR spectroscopy of $\mathbf{3 z}$. 
d) $1 \mathrm{H}$ and 13C NMR spectra of products in oxidative cross-coupling

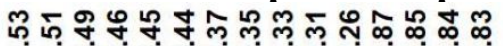

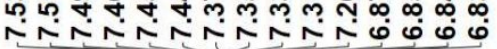

$\mathrm{H}_{3} \mathrm{C}$ œ
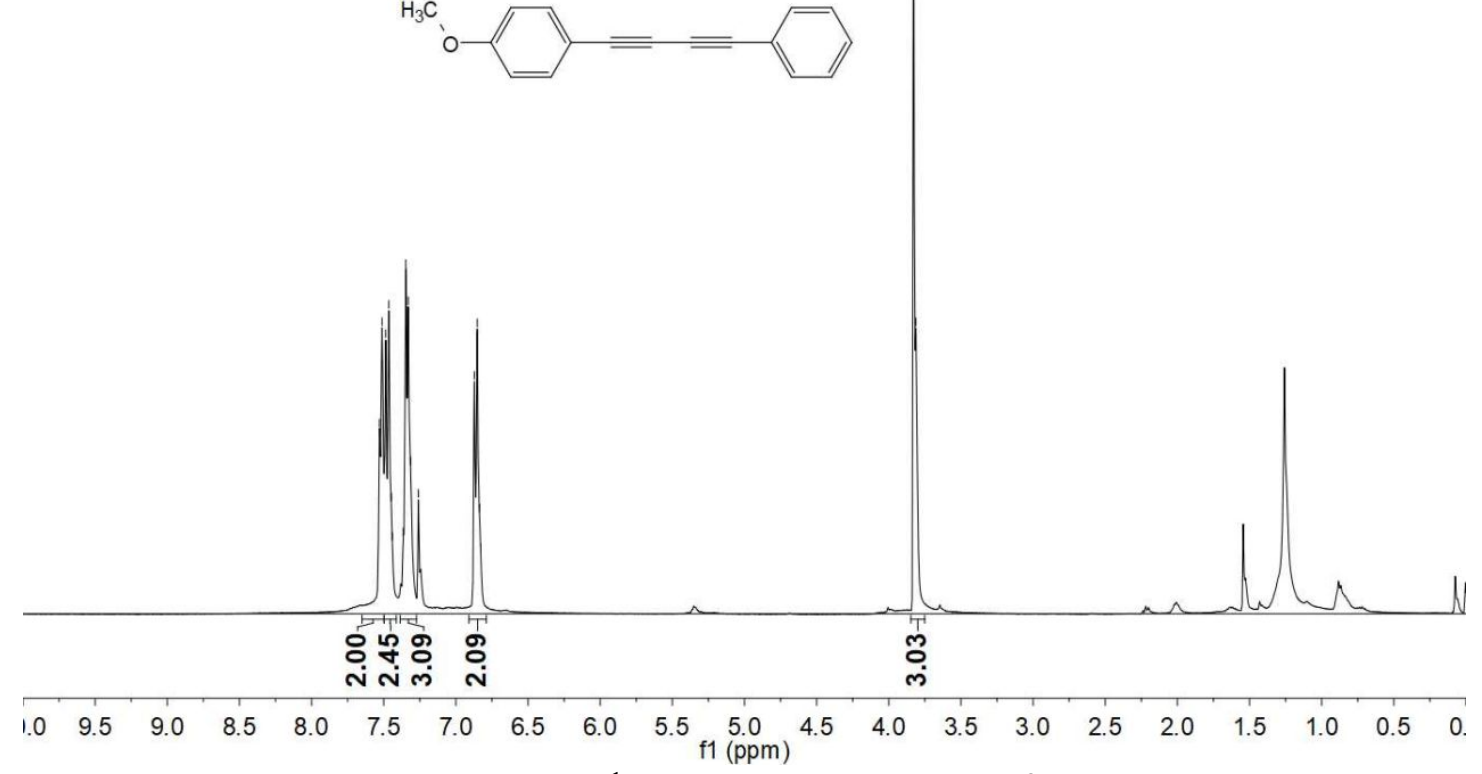

Figure S65. ${ }^{1} \mathrm{H}$ NMR spectroscopy of $\mathbf{4 a}$.

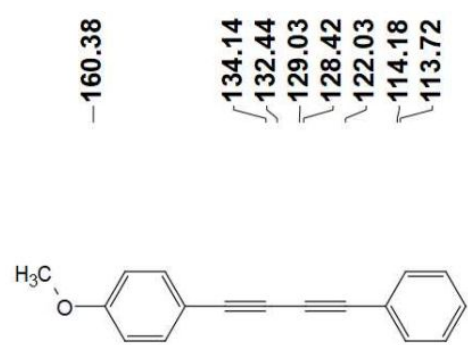

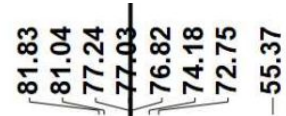

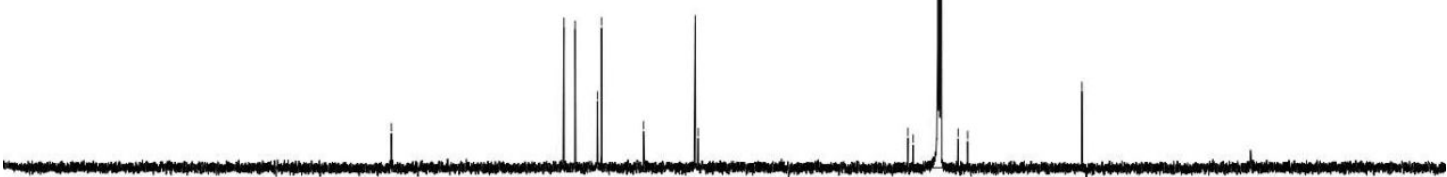

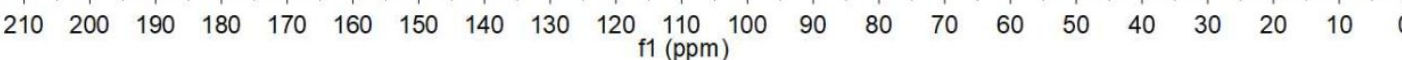

Figure S66. ${ }^{13} \mathrm{C}$ NMR spectroscopy of $\mathbf{4 a}$. 


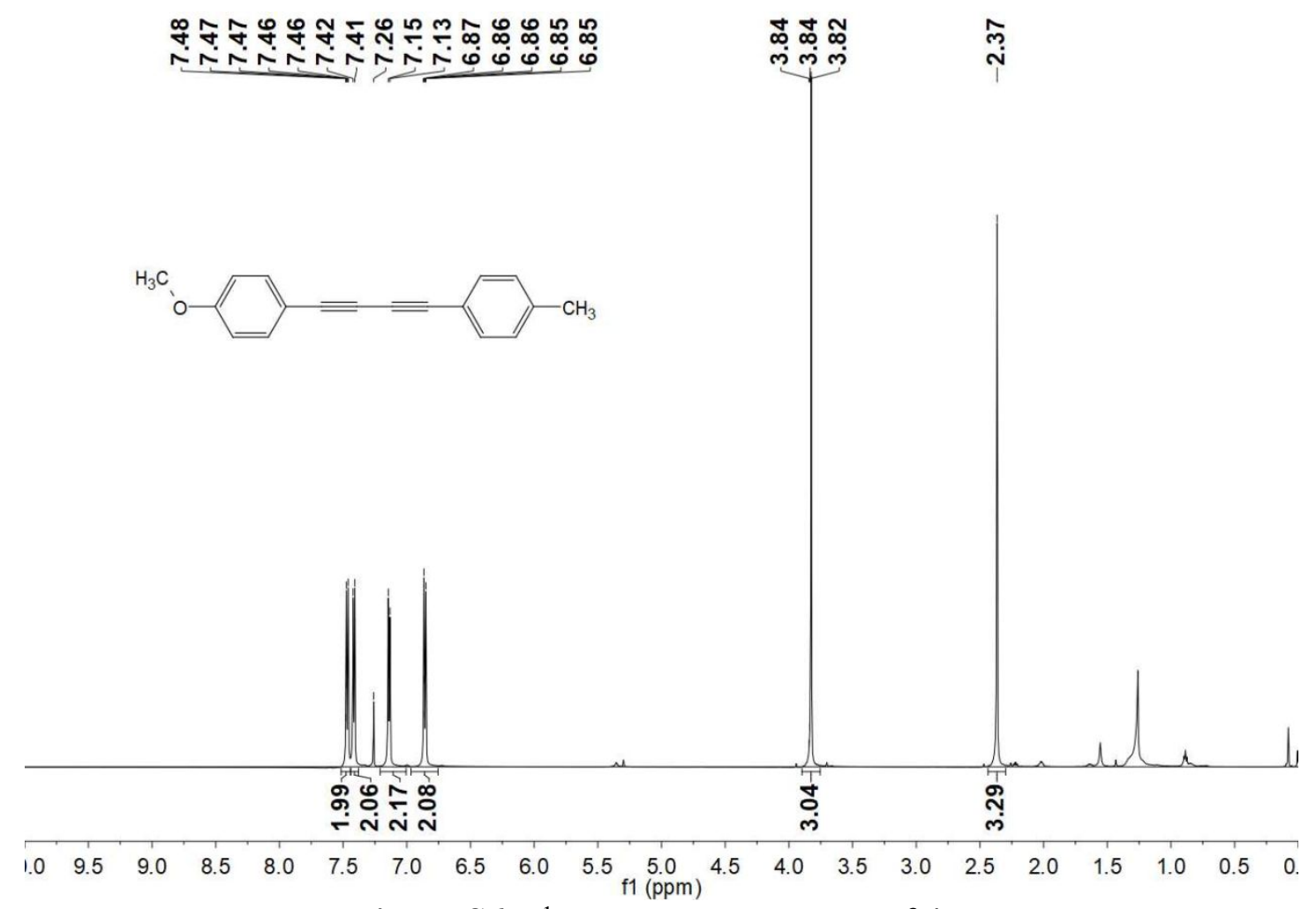

Figure S67. ${ }^{1} \mathrm{H}$ NMR spectroscopy of $\mathbf{4 b}$.

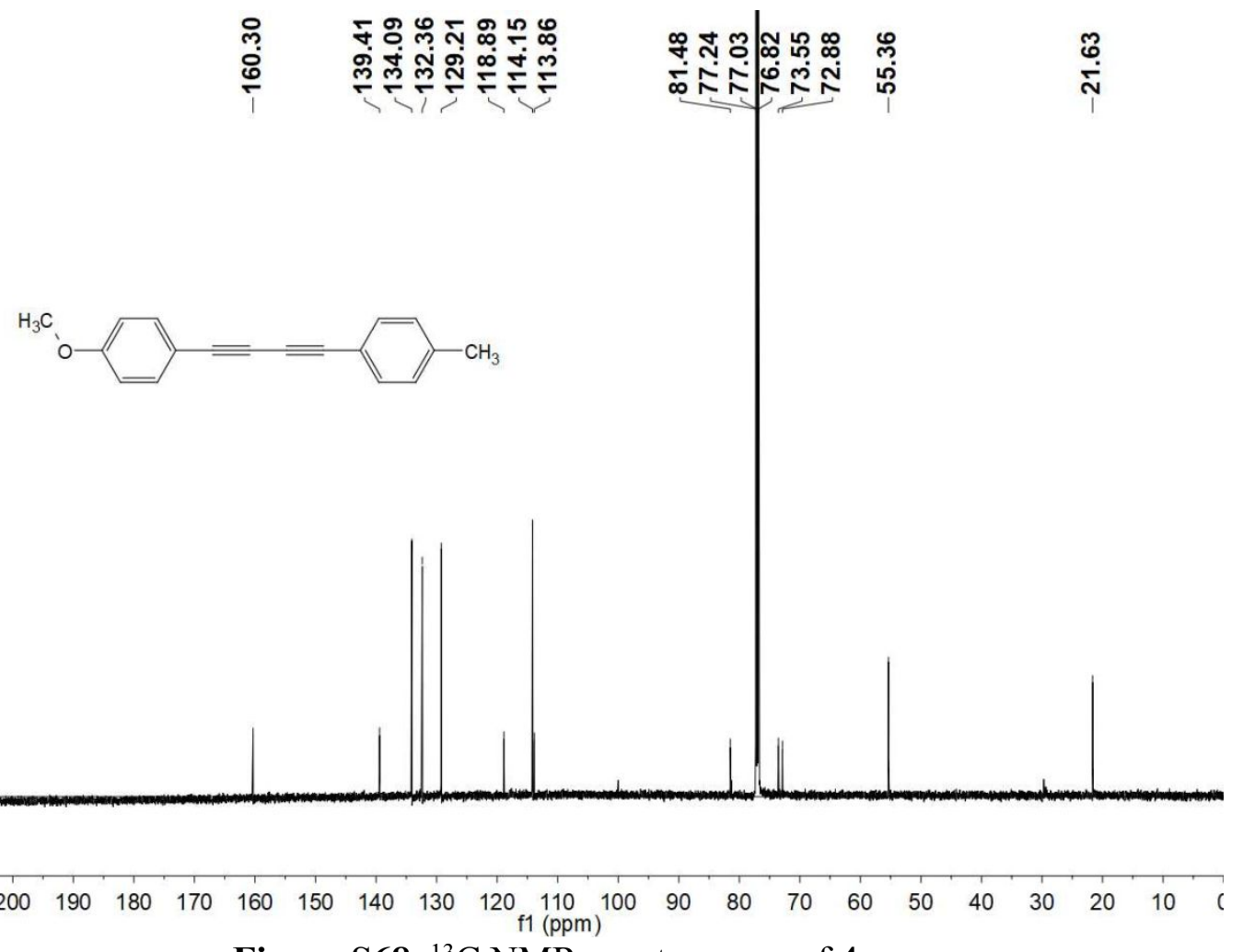

Figure S68. ${ }^{13} \mathrm{C}$ NMR spectroscopy of $\mathbf{4 a}$. 


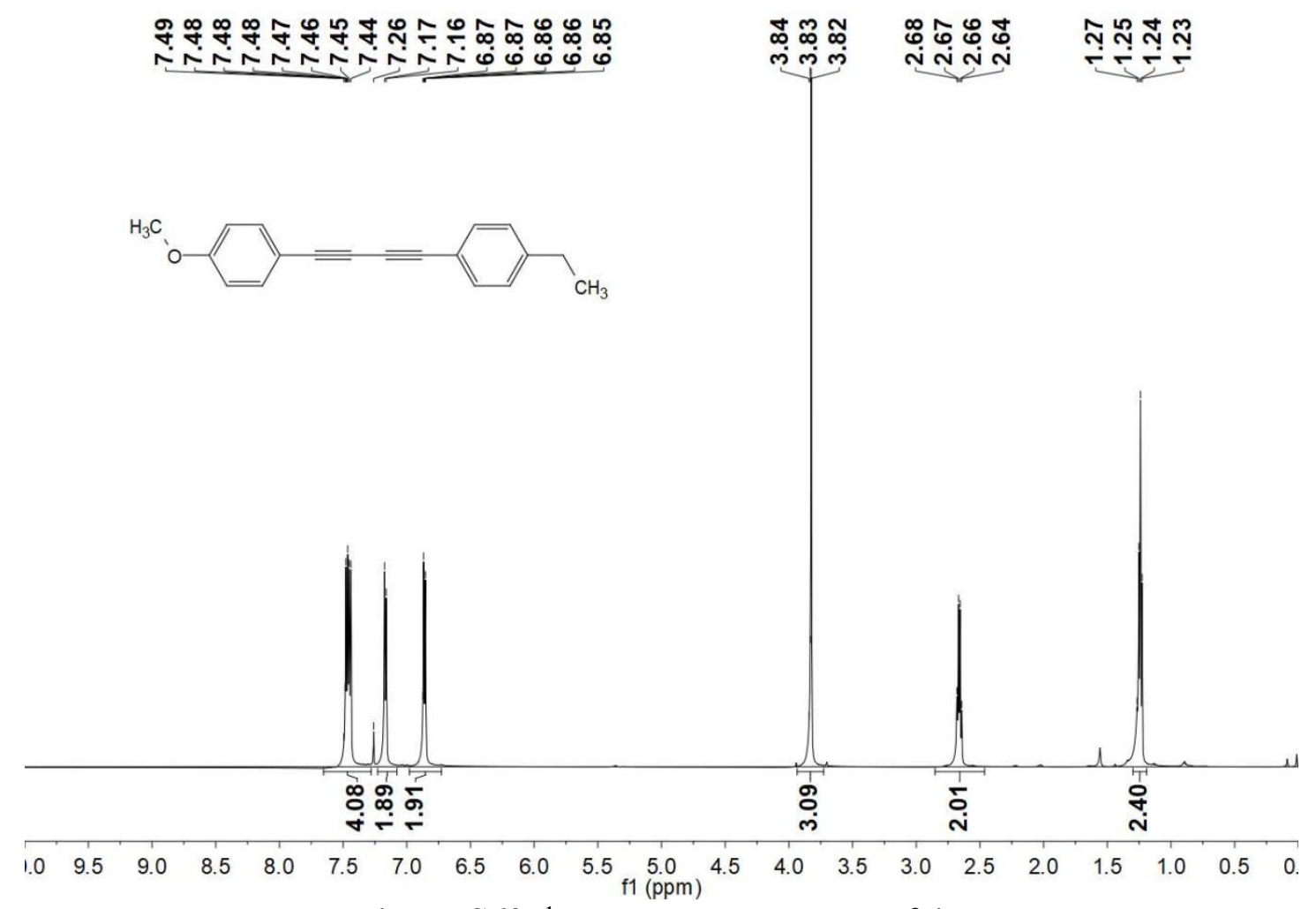

Figure S69. ${ }^{1} \mathrm{H}$ NMR spectroscopy of $\mathbf{4 c}$.

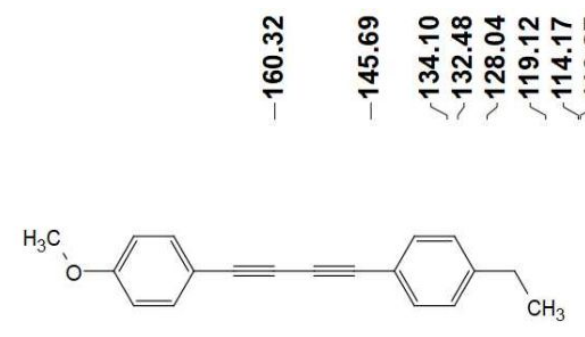

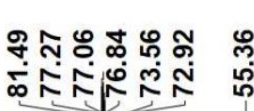

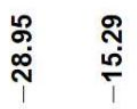

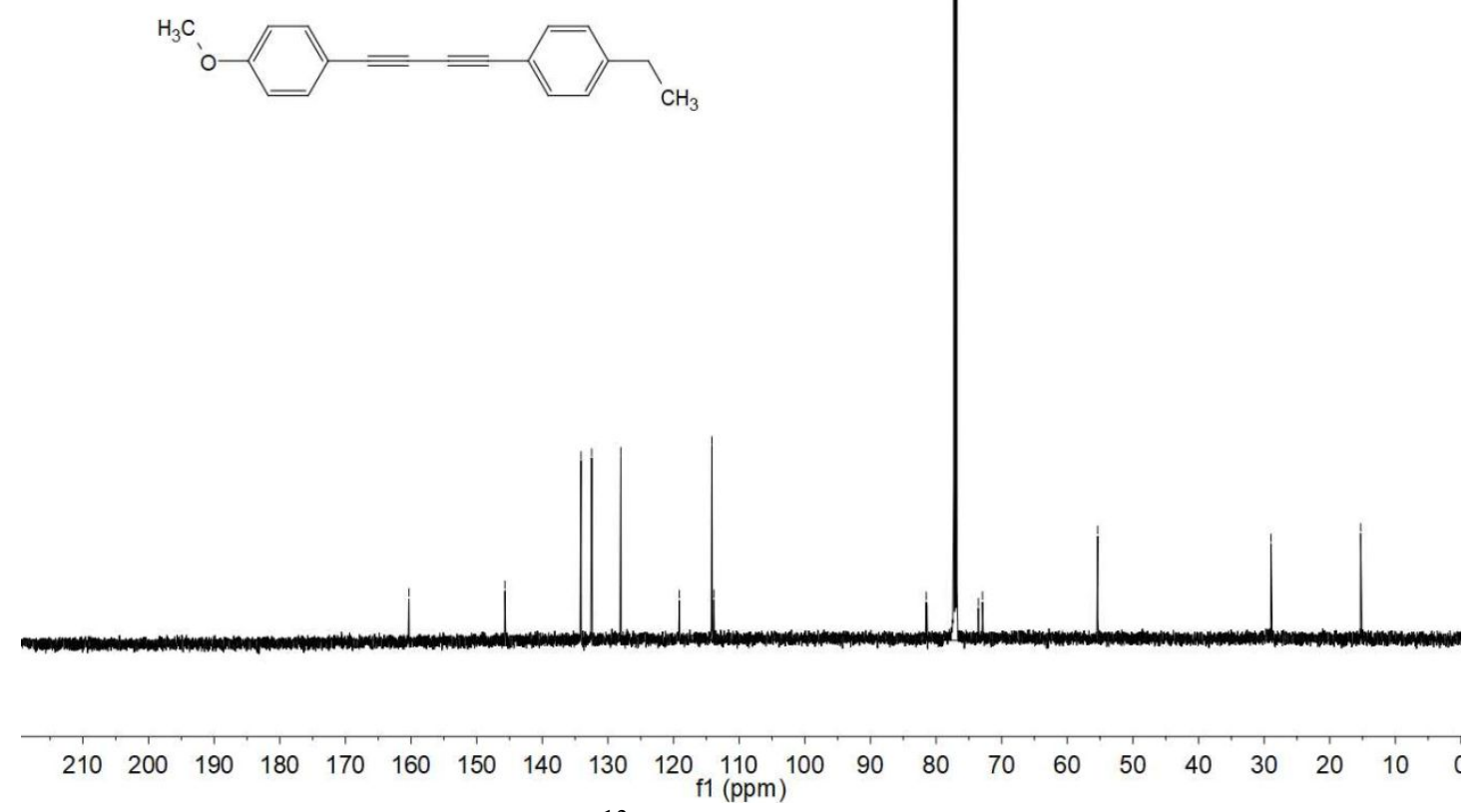

Figure S70. ${ }^{13} \mathrm{C}$ NMR spectroscopy of $4 \mathbf{c}$. 


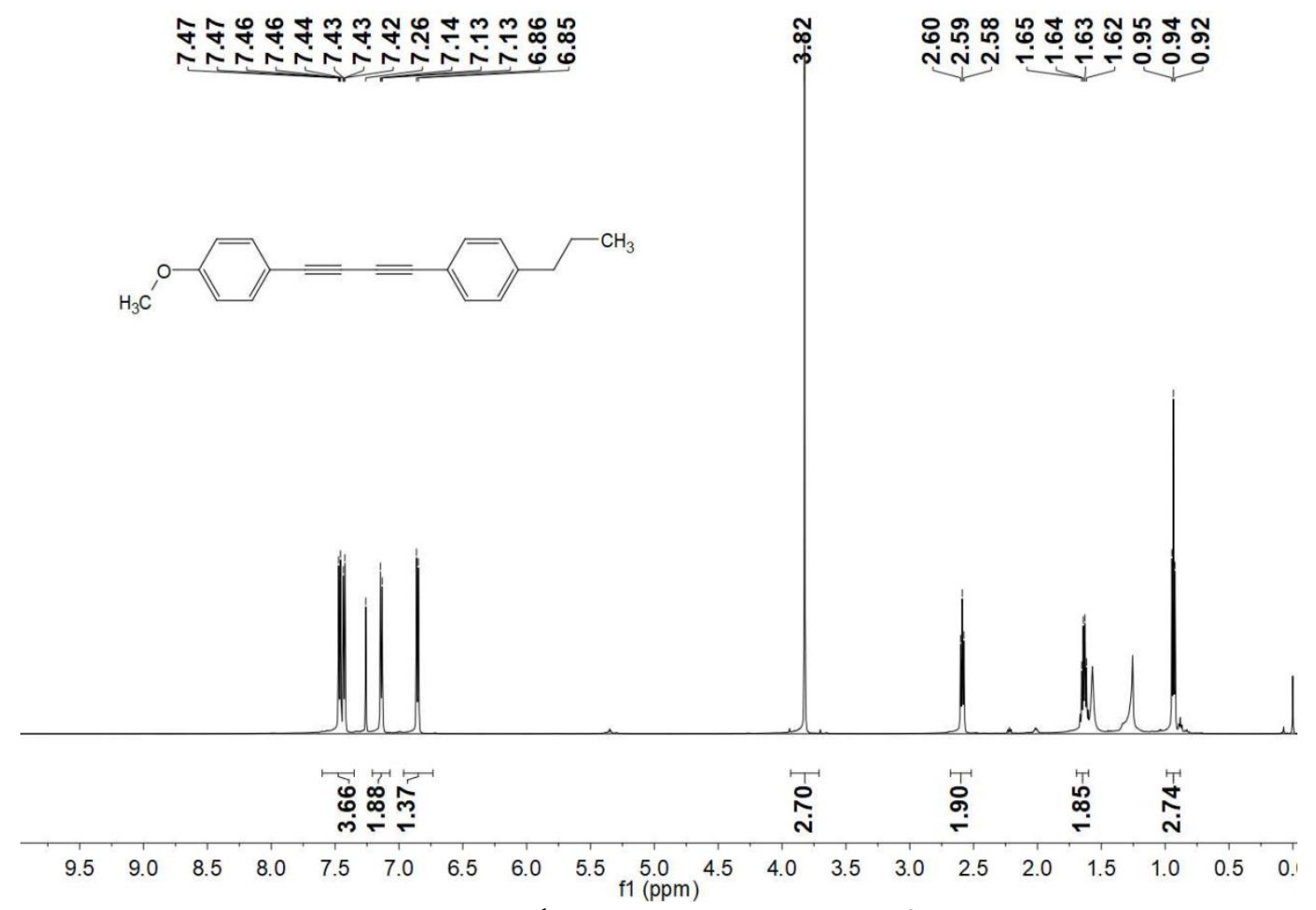

Figure S71. ${ }^{1} \mathrm{H}$ NMR spectroscopy of $\mathbf{4 d}$.

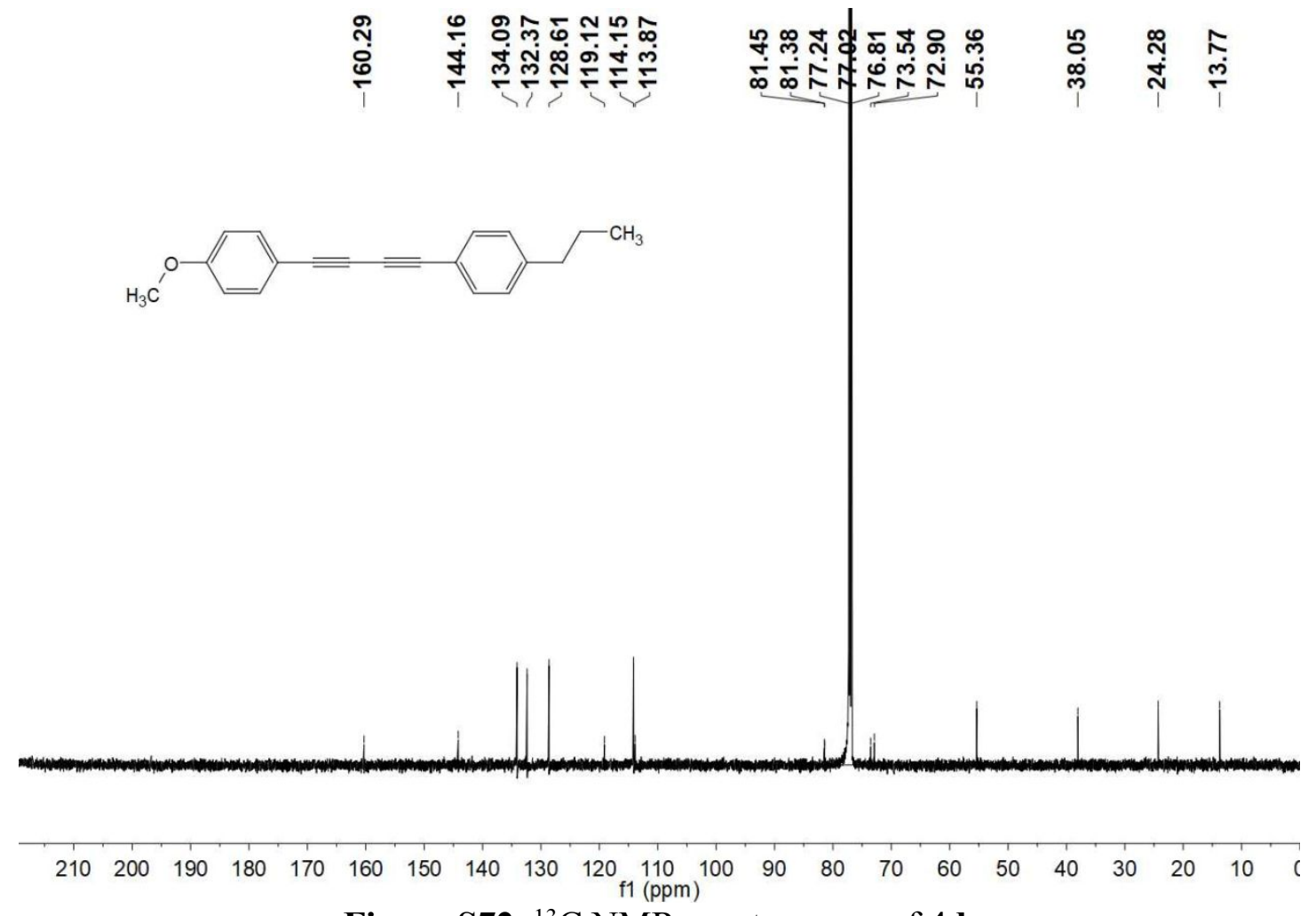

Figure S72. ${ }^{13} \mathrm{C}$ NMR spectroscopy of $\mathbf{4 d}$. 


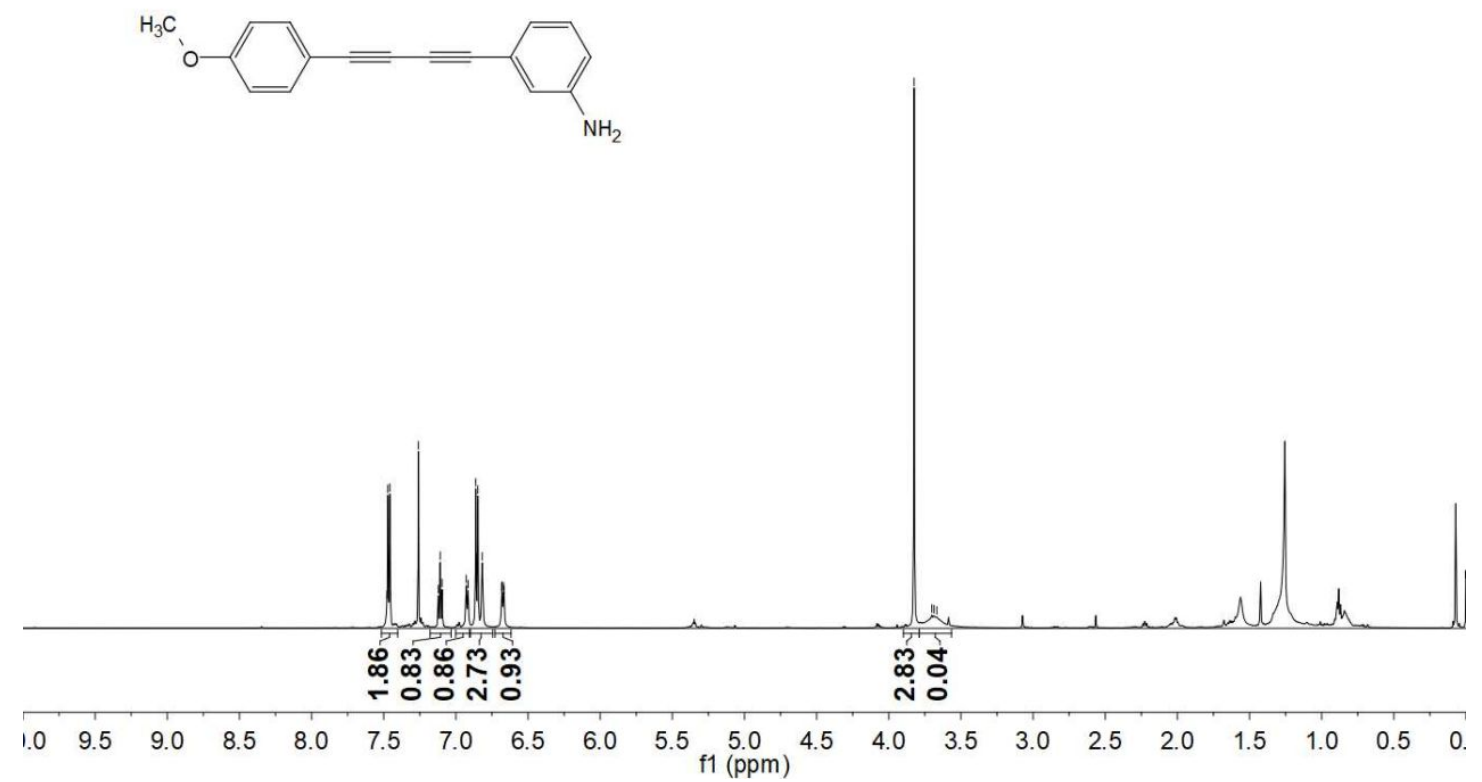

Figure S73. ${ }^{1} \mathrm{H}$ NMR spectroscopy of $4 \mathbf{e}$.

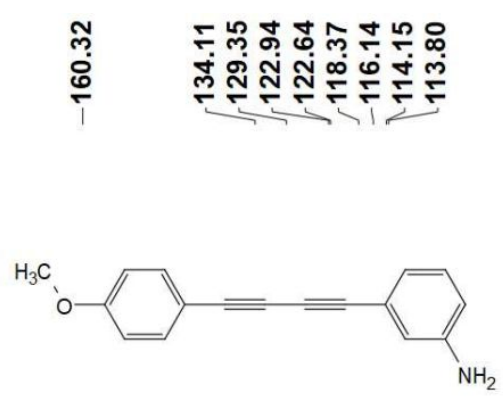

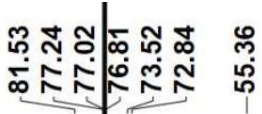

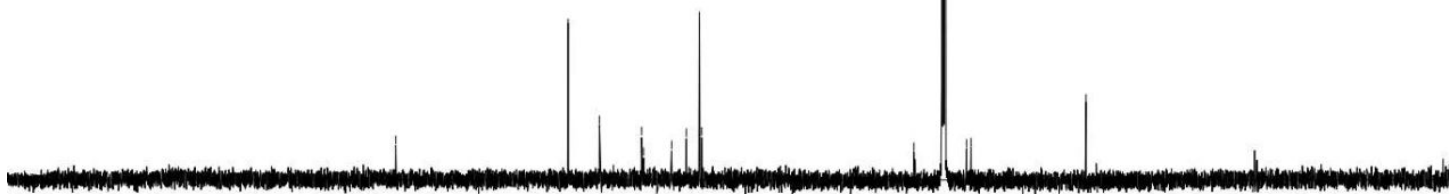

$\begin{array}{lllllllllllllllllllll}210 & 200 & 190 & 180 & 170 & 160 & 150 & 140 & 130 & 120 & \begin{array}{c}110 \\ \mathrm{f} 1(\mathrm{ppm})\end{array} & 100 & 90 & 80 & 70 & 60 & 50 & 40 & 30 & 20 & 10\end{array}$

Figure S74. ${ }^{13} \mathrm{C}$ NMR spectroscopy of 4 e. 


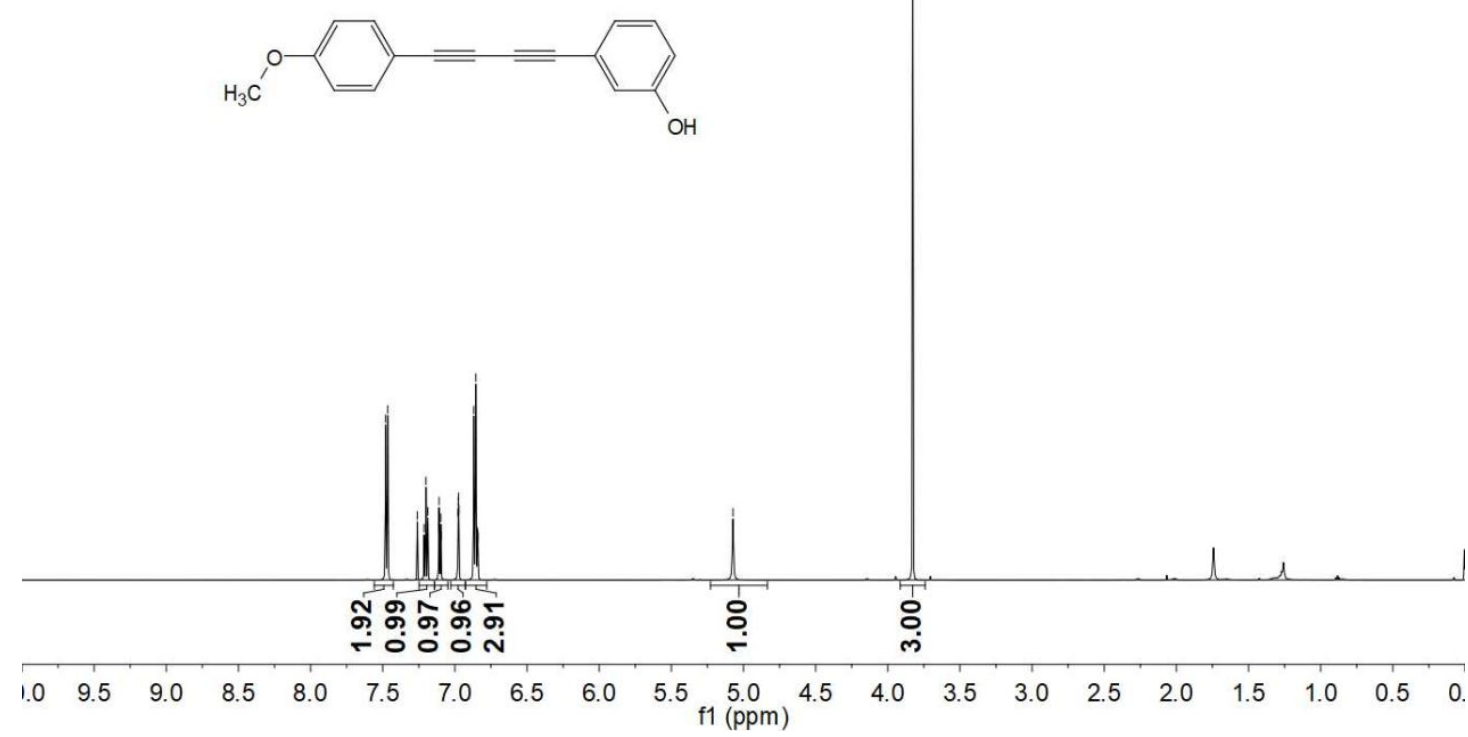

Figure S75. ${ }^{1} \mathrm{H}$ NMR spectroscopy of $\mathbf{4 f}$.

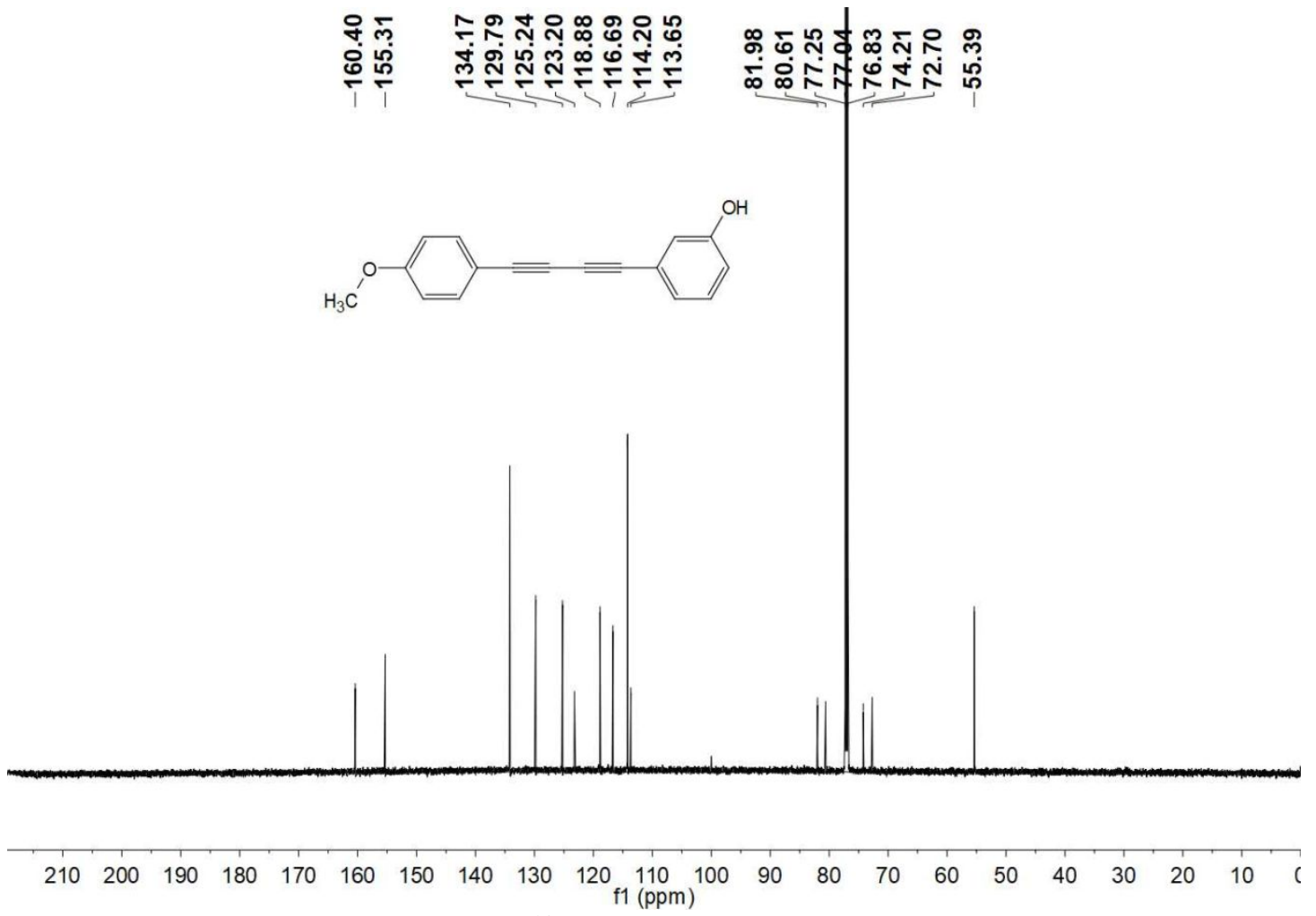

Figure S76. ${ }^{13} \mathrm{C}$ NMR spectroscopy of $\mathbf{4 f}$. 


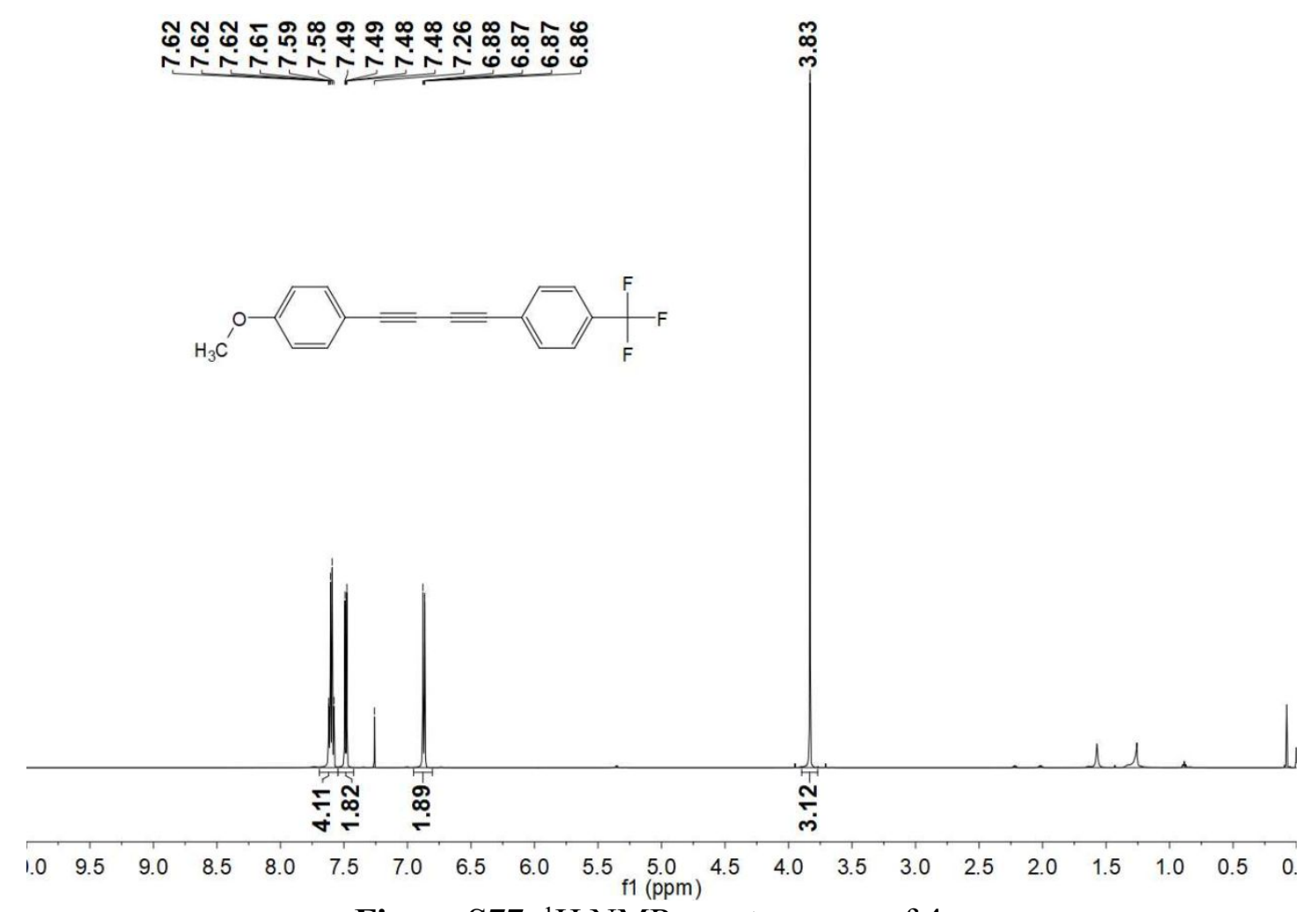

Figure S77. ${ }^{1} \mathrm{H}$ NMR spectroscopy of $\mathbf{4 g}$.

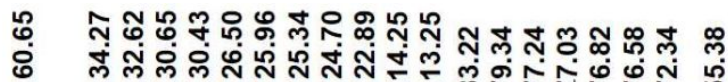

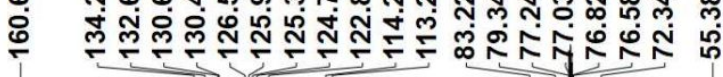
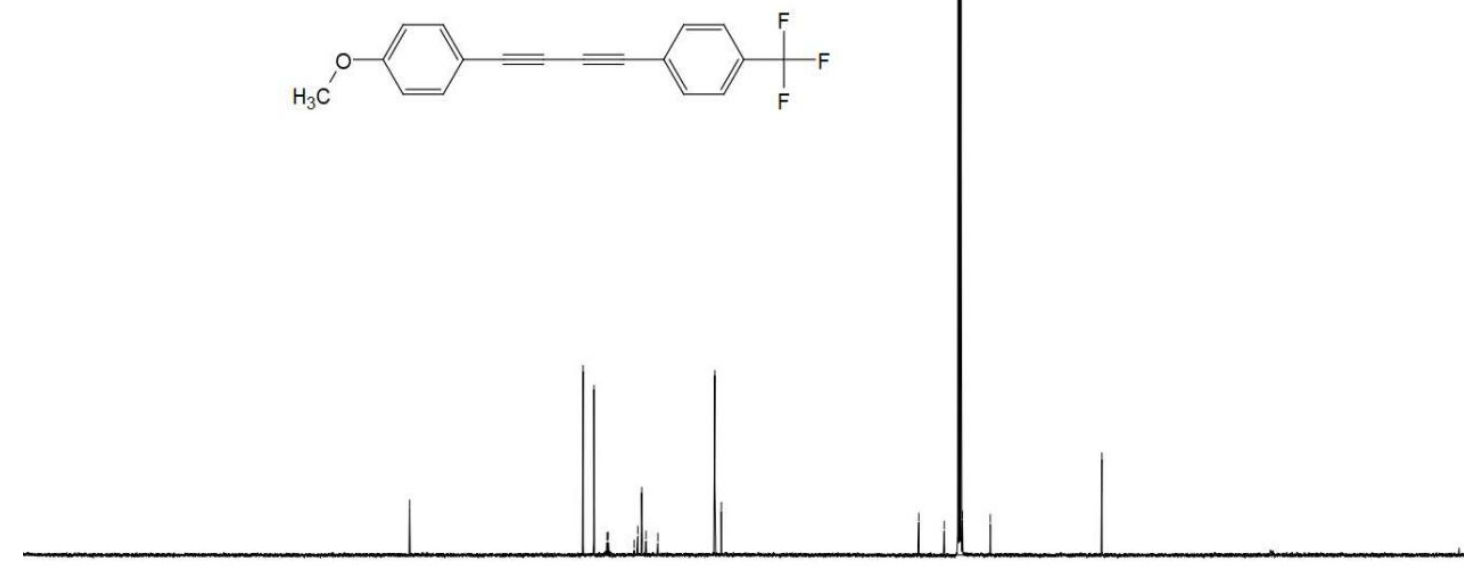

$\begin{array}{llllllllllllllllllllllll}210 & 200 & 190 & 180 & 170 & 160 & 150 & 140 & 130 & 120 & \begin{array}{c}110 \\ \mathrm{f} 1(\mathrm{ppm})\end{array} & 100 & 90 & 80 & 70 & 60 & 50 & 40 & 30 & 20 & 10 & (\end{array}$

Figure S78. ${ }^{13} \mathrm{C}$ NMR spectroscopy of $\mathbf{4 g}$. 


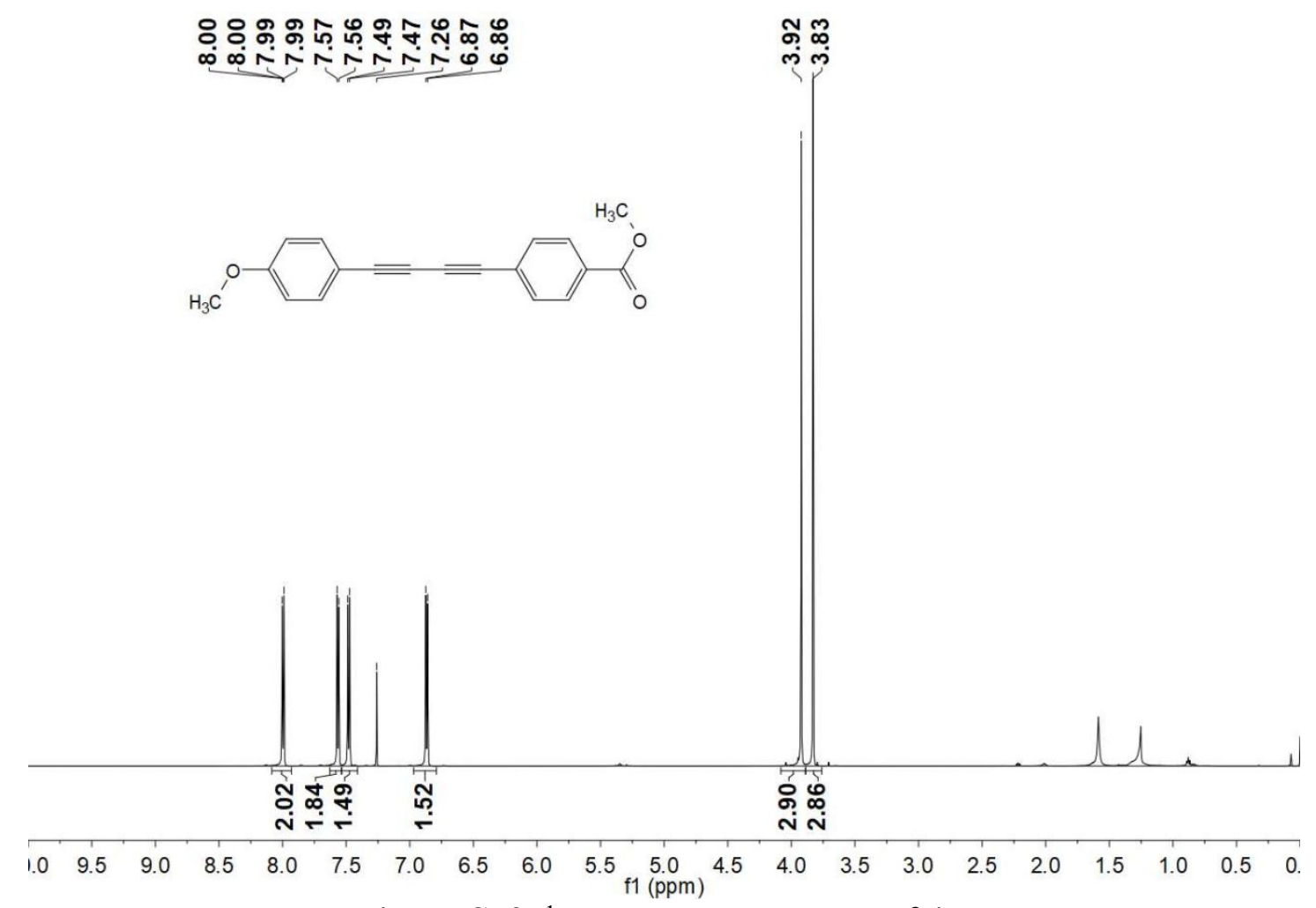

Figure S79. ${ }^{1} \mathrm{H}$ NMR spectroscopy of $\mathbf{4 h}$.

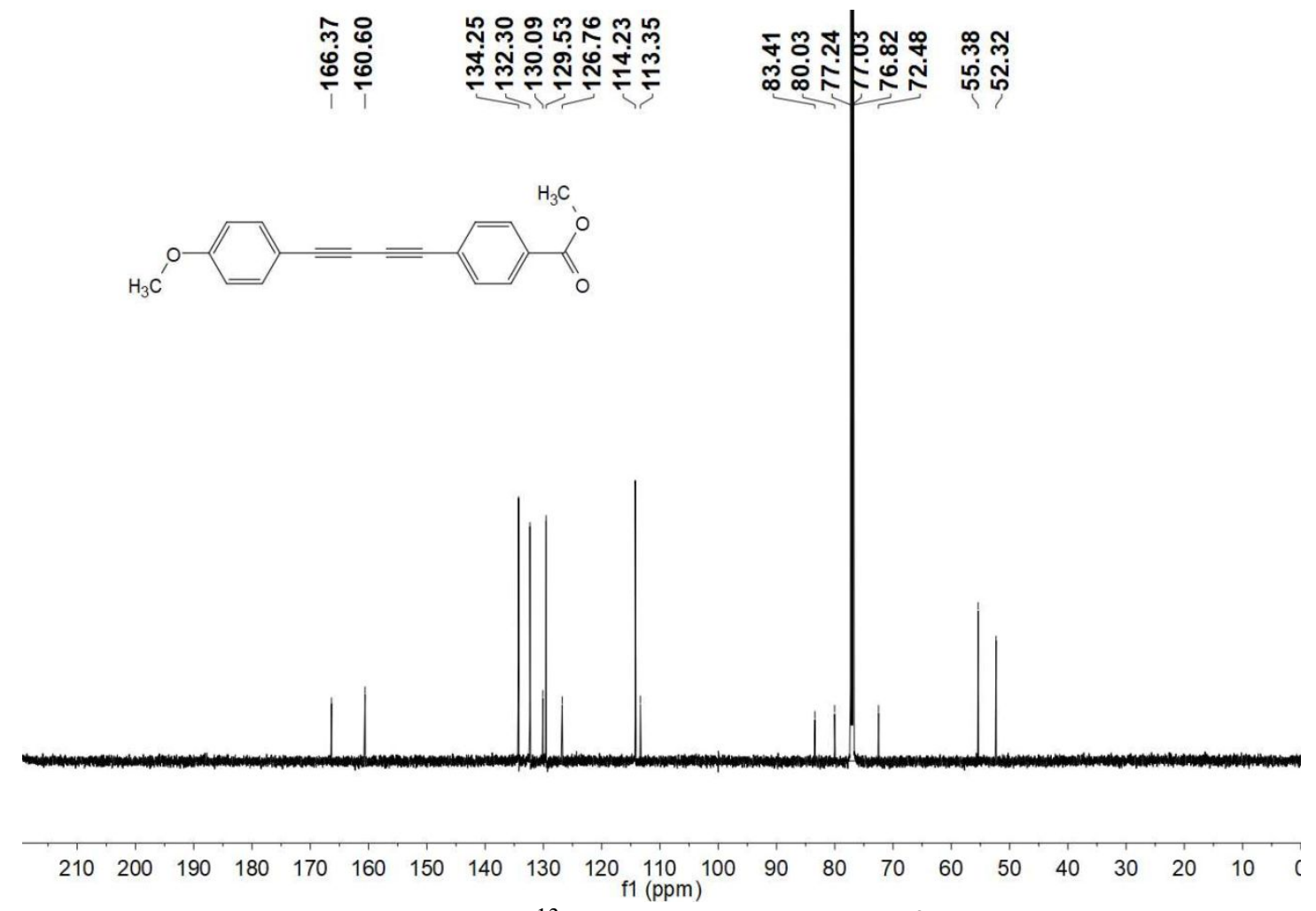

Figure S80. ${ }^{13} \mathrm{C}$ NMR spectroscopy of $\mathbf{4 h}$. 


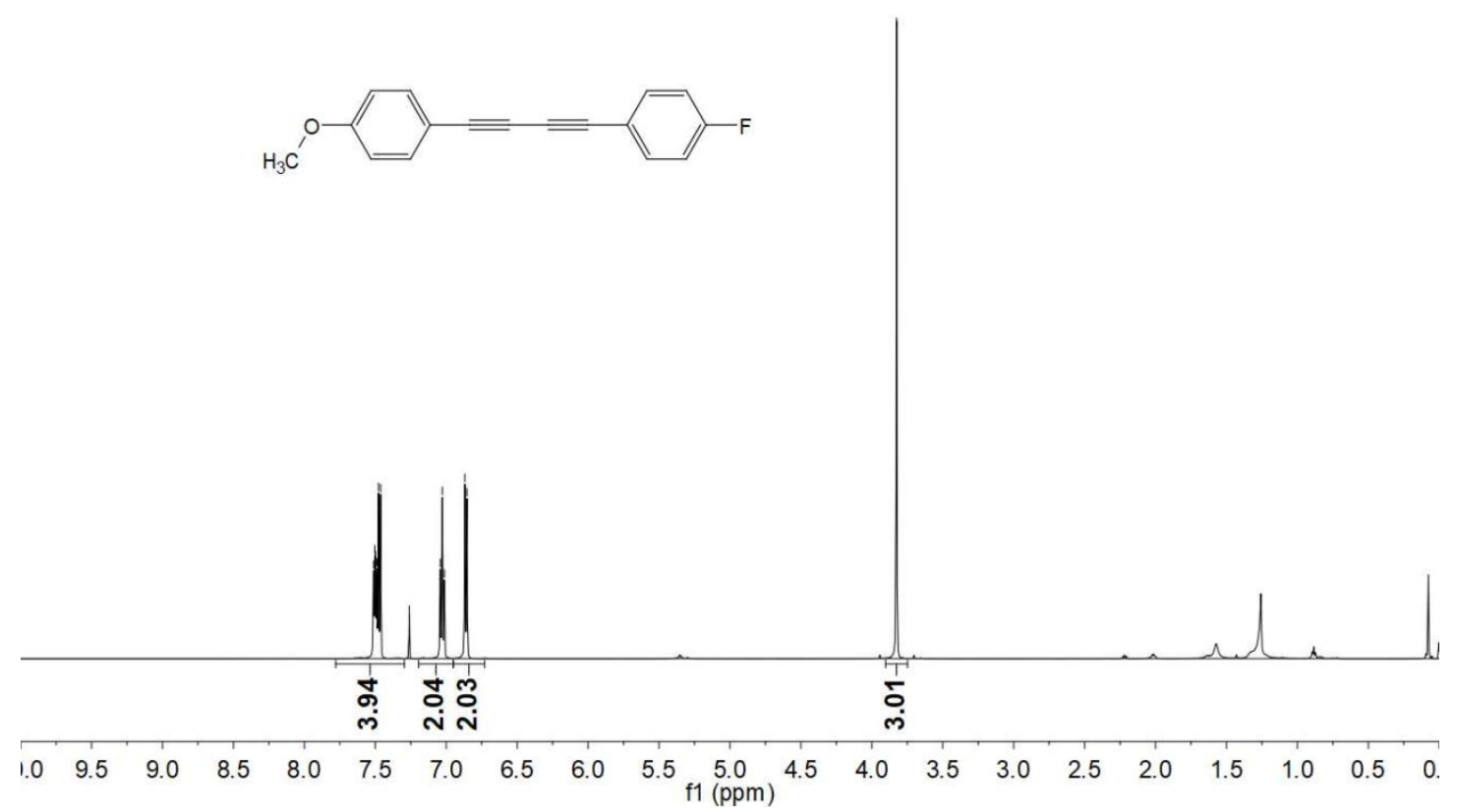

Figure S81. ${ }^{1} \mathrm{H}$ NMR spectroscopy of $\mathbf{4 i}$.

ำㅇำ 눈

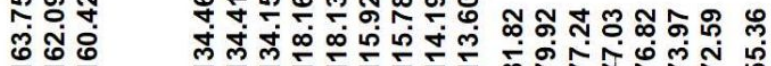

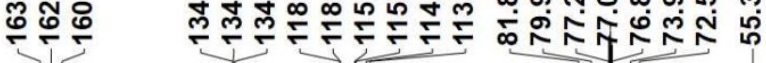
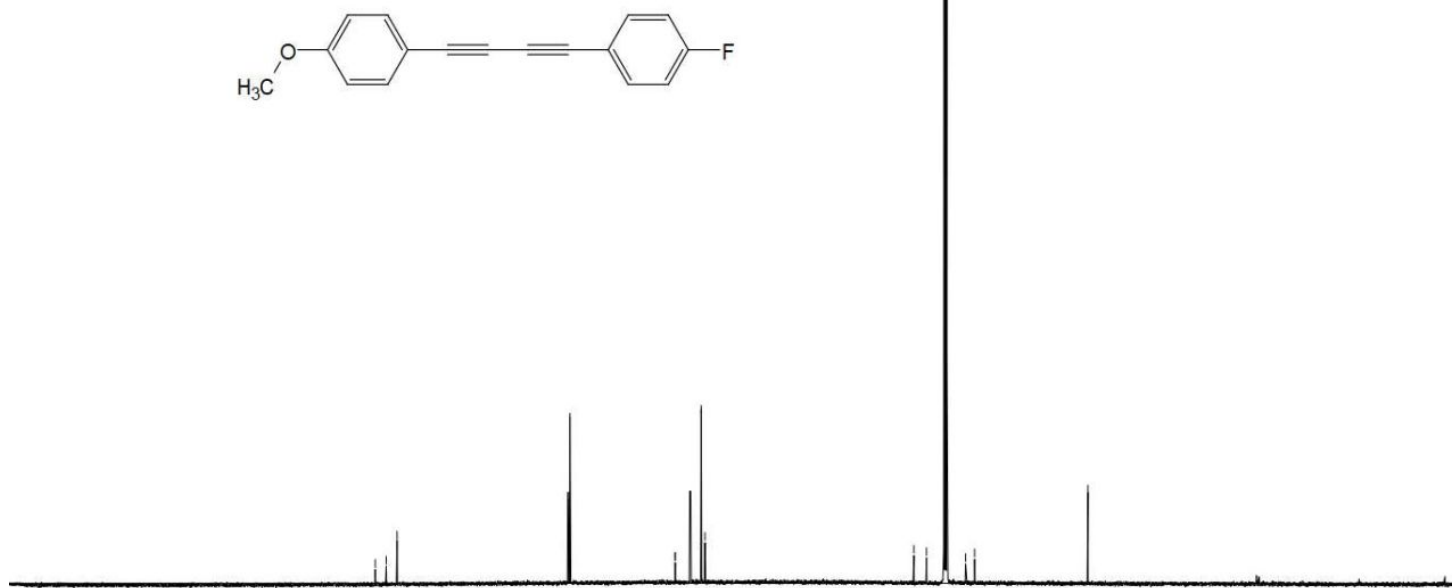

$\begin{array}{lllllllllllllllllllll}210 & 200 & 190 & 180 & 170 & 160 & 150 & 140 & 130 & 120 & 110 & 100 & 90 & 80 & 70 & 60 & 50 & 40 & 30 & 20 & 10\end{array}$

Figure S82. ${ }^{13} \mathrm{C}$ NMR spectroscopy of $4 \mathbf{i}$. 


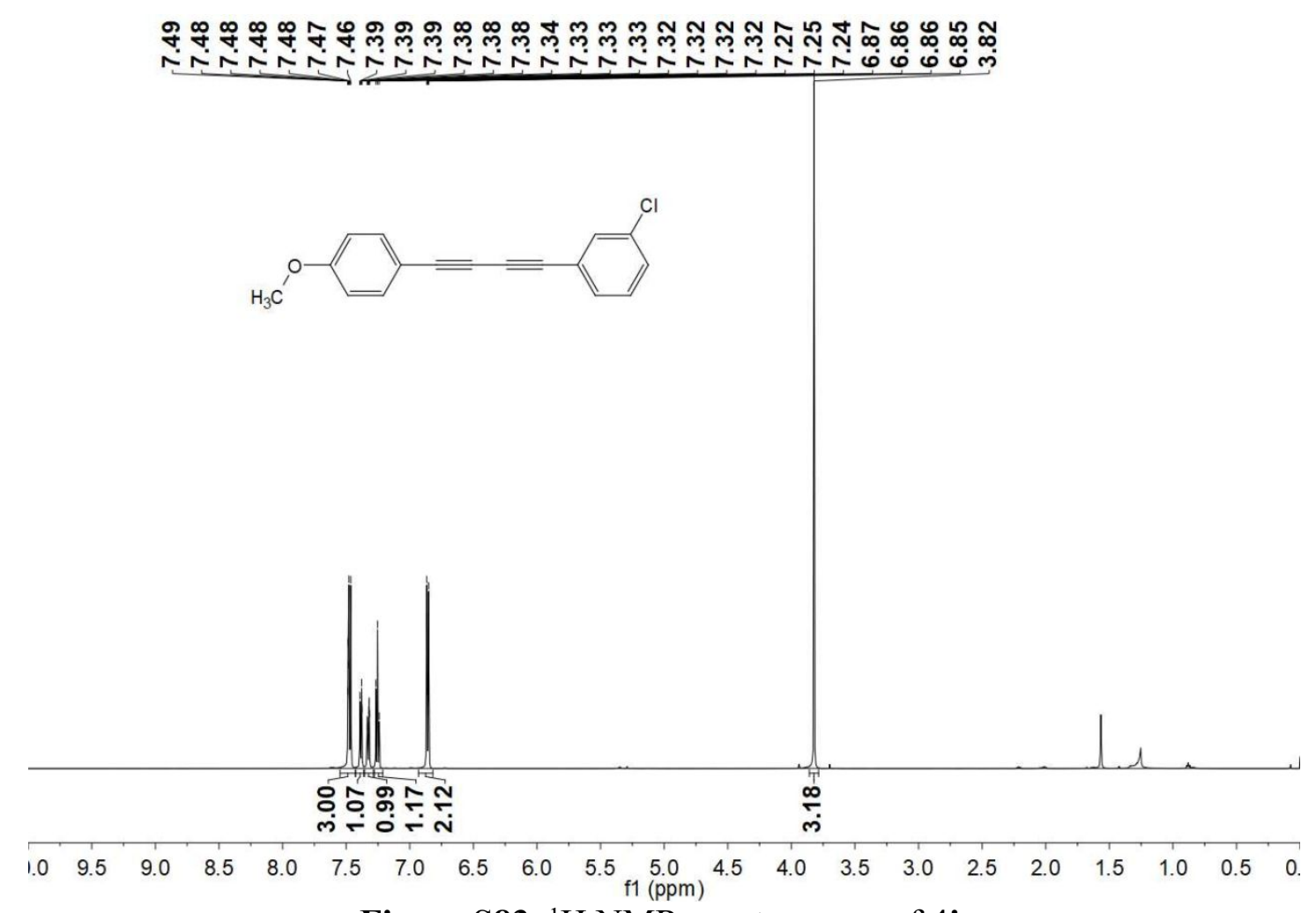

Figure S83. ${ }^{1} \mathrm{H}$ NMR spectroscopy of $\mathbf{4 j}$.

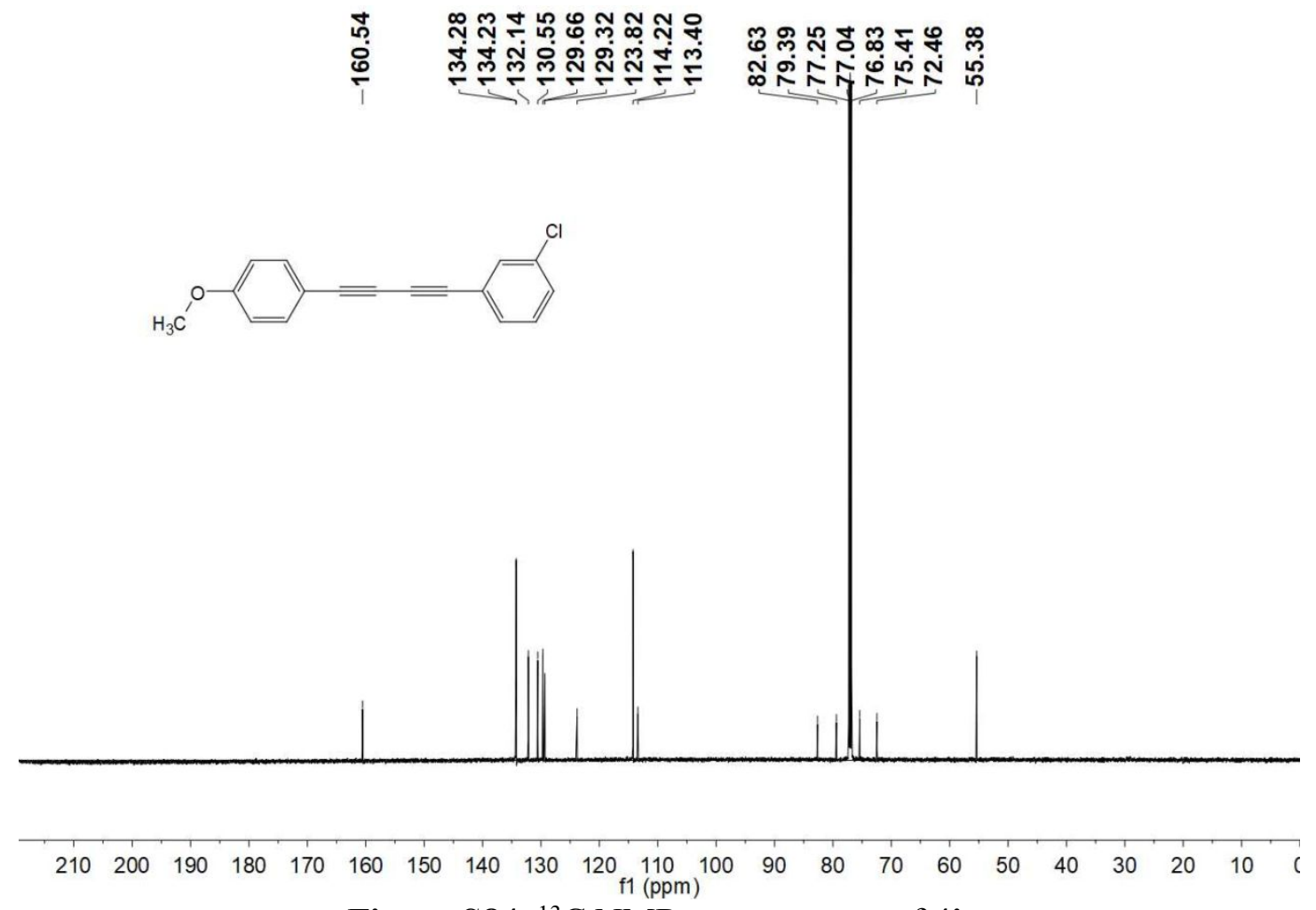

Figure S84. ${ }^{13} \mathrm{C}$ NMR spectroscopy of $\mathbf{4 j}$. 


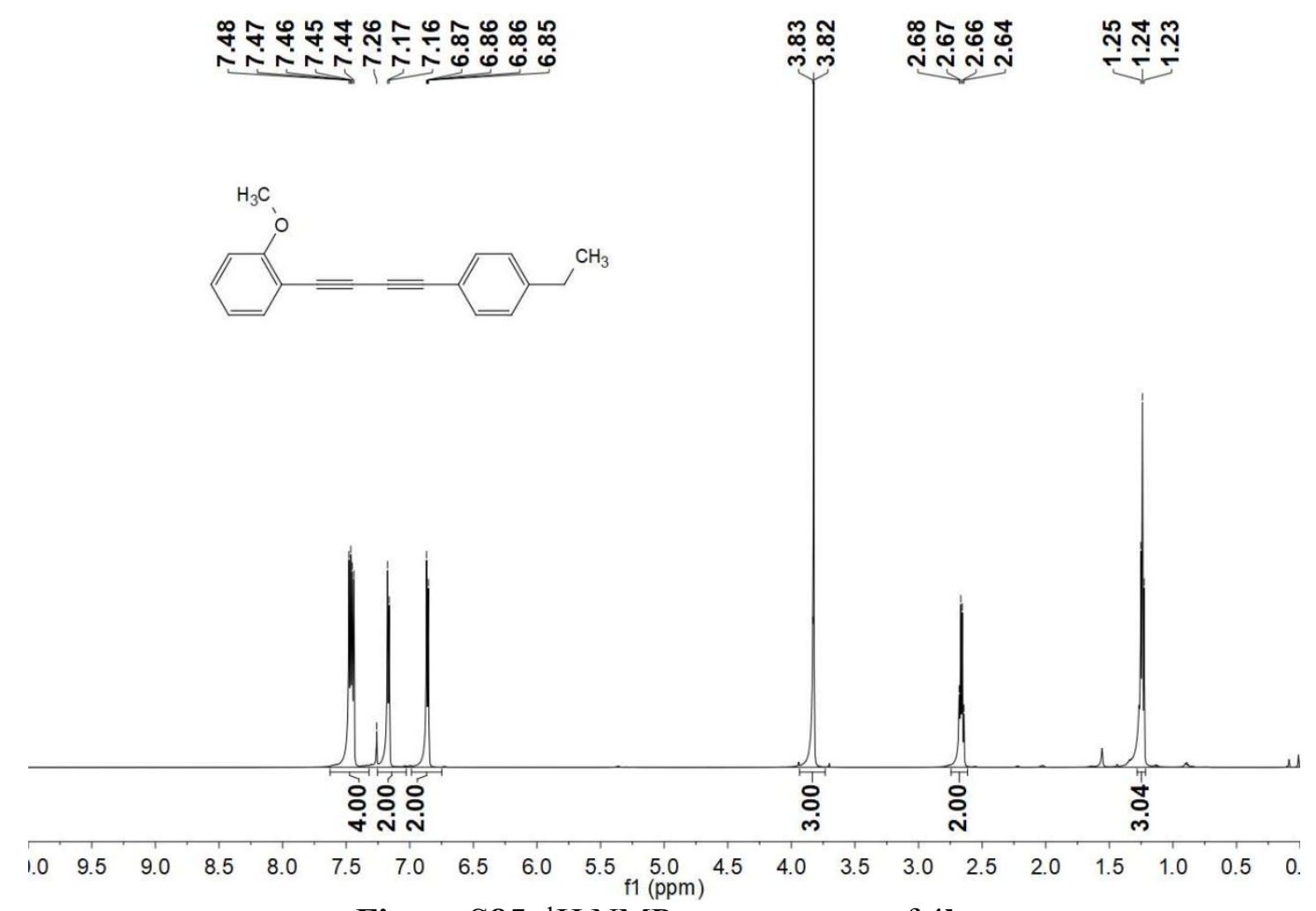

Figure S85. ${ }^{1} \mathrm{H}$ NMR spectroscopy of $\mathbf{4 k}$.

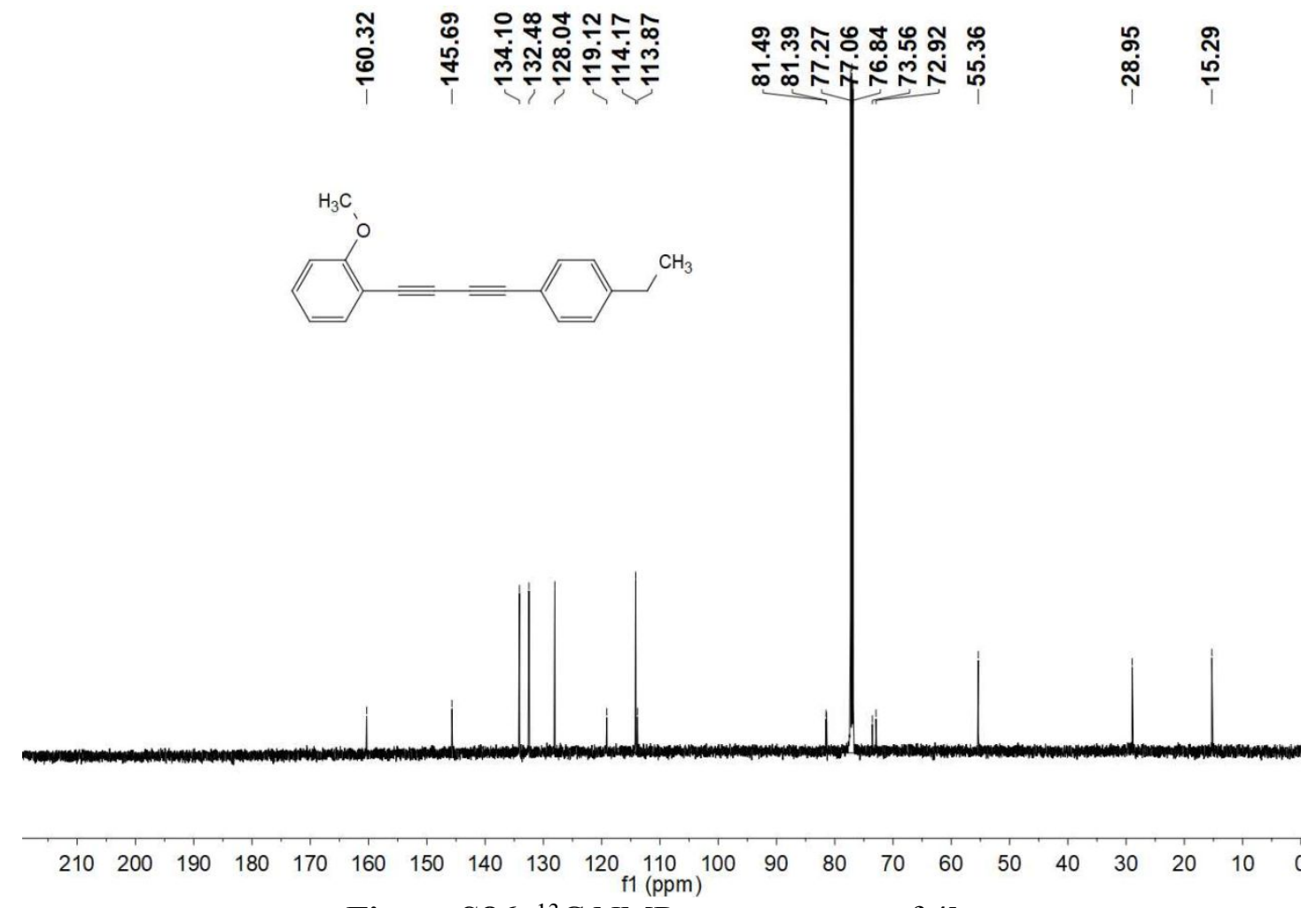

Figure S86. ${ }^{13} \mathrm{C}$ NMR spectroscopy of $\mathbf{4 k}$. 

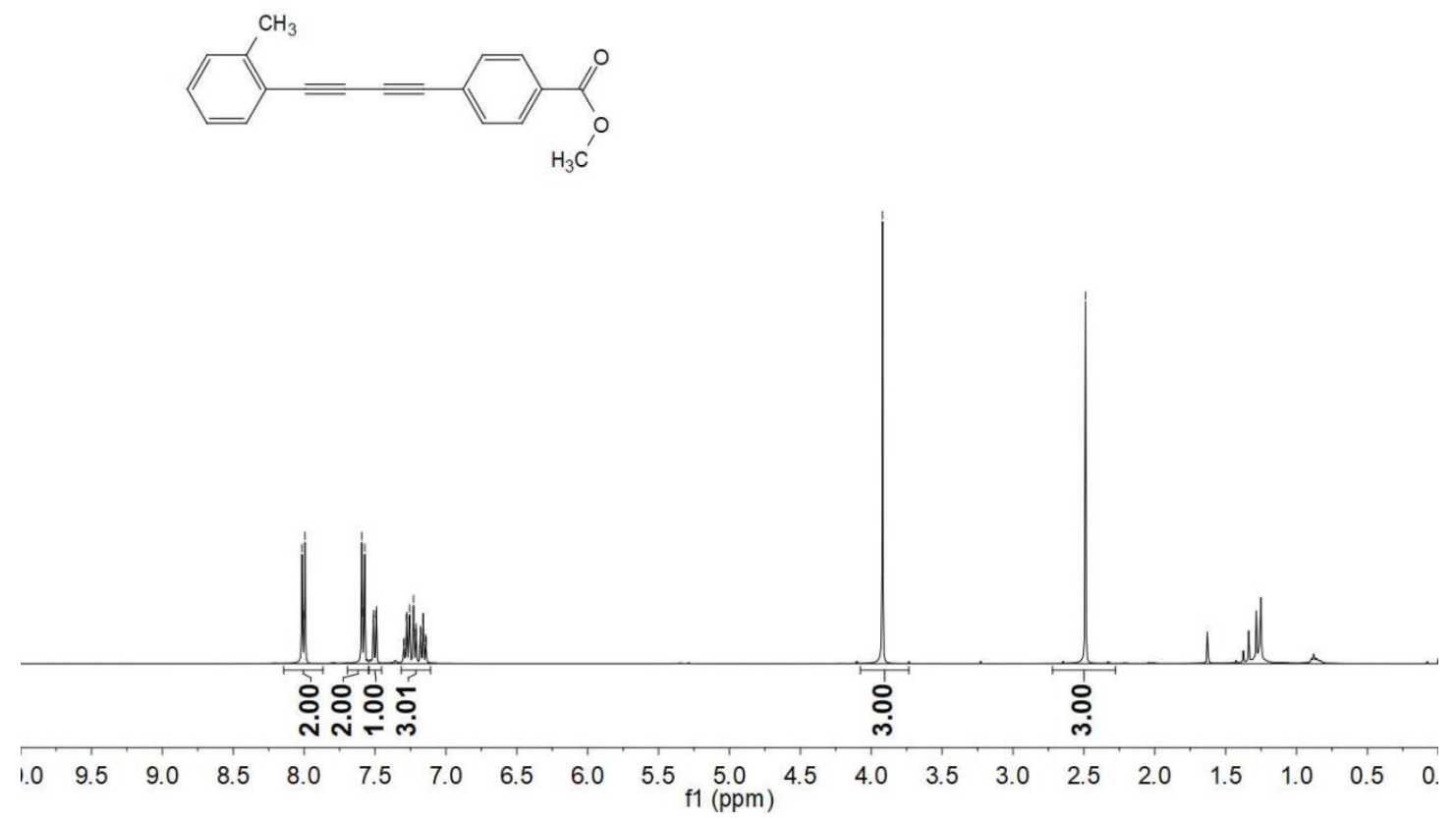

Figure S87. ${ }^{1} \mathrm{H}$ NMR spectroscopy of $\mathbf{4 l}$.

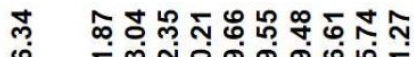

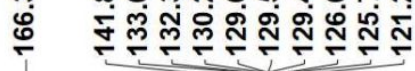

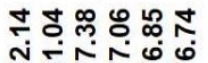

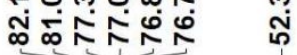

กั่
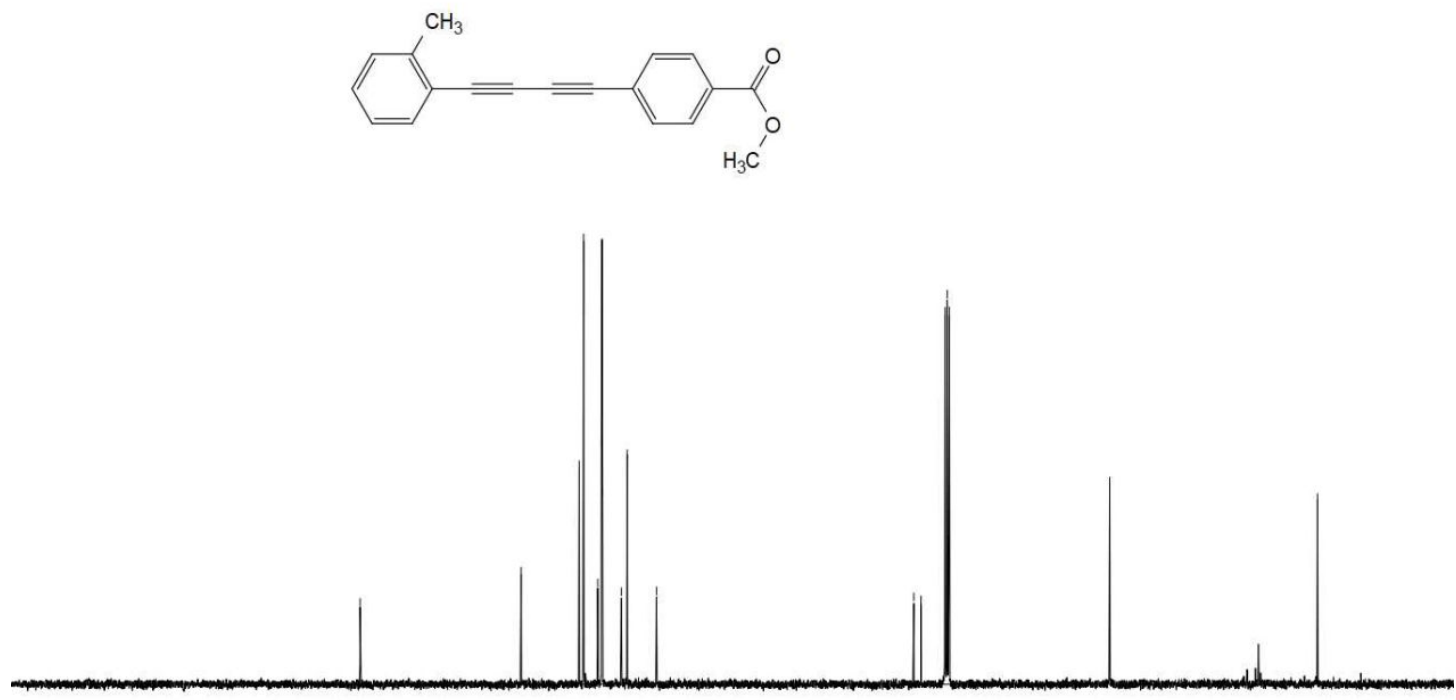

$\begin{array}{lllllllllllllllllllll}210 & 200 & 190 & 180 & 170 & 160 & 150 & 140 & 130 & 120 & 110 & 100 & 90 & 80 & 70 & 60 & 50 & 40 & 30 & 20 & 10\end{array}$

Figure S88. ${ }^{13} \mathrm{C}$ NMR spectroscopy of 41 . 


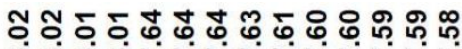

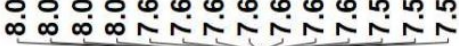
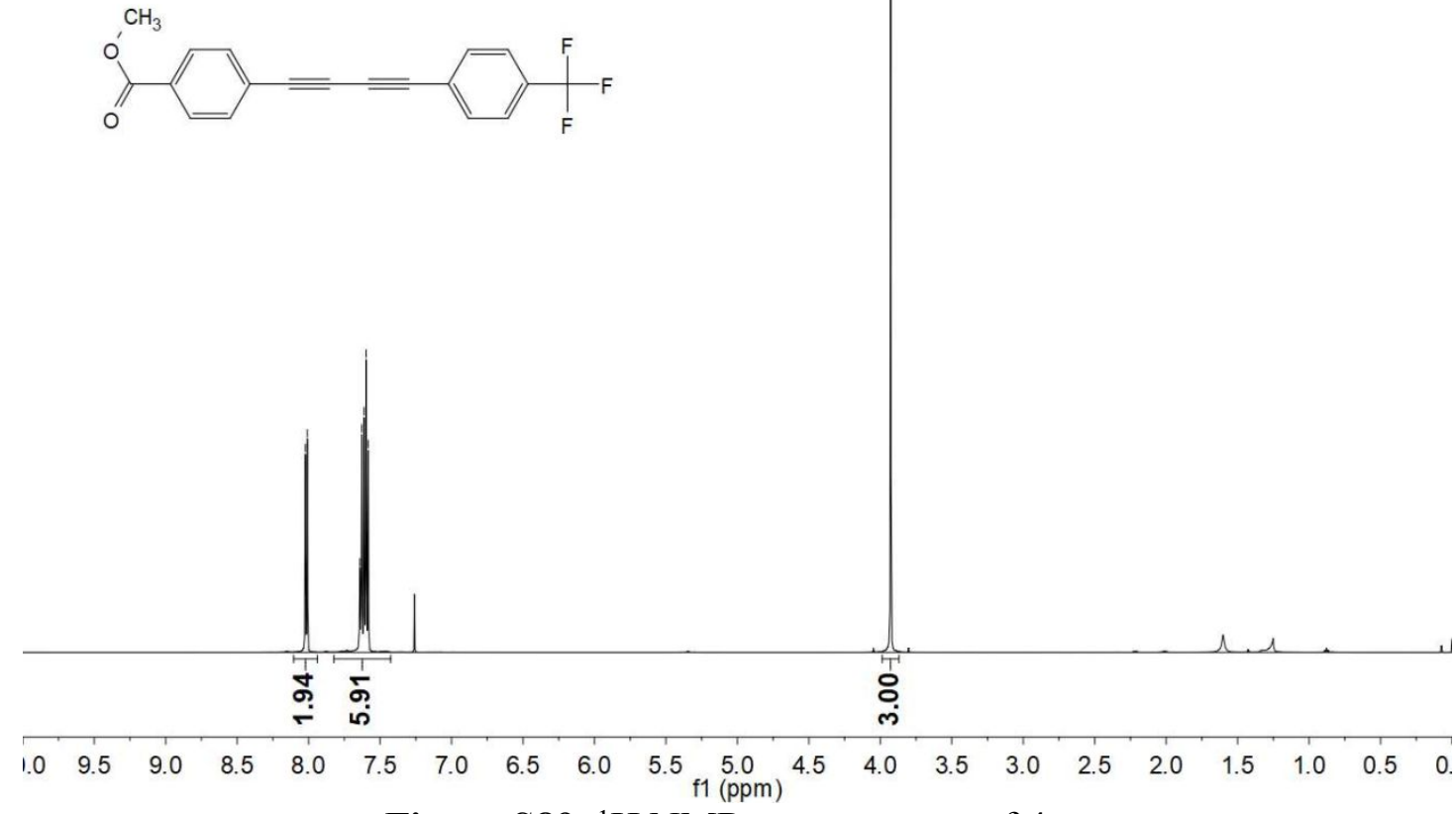

Figure S89. ${ }^{1} \mathrm{H}$ NMR spectroscopy of $\mathbf{4 m}$.

궁ำ

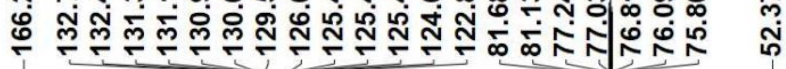
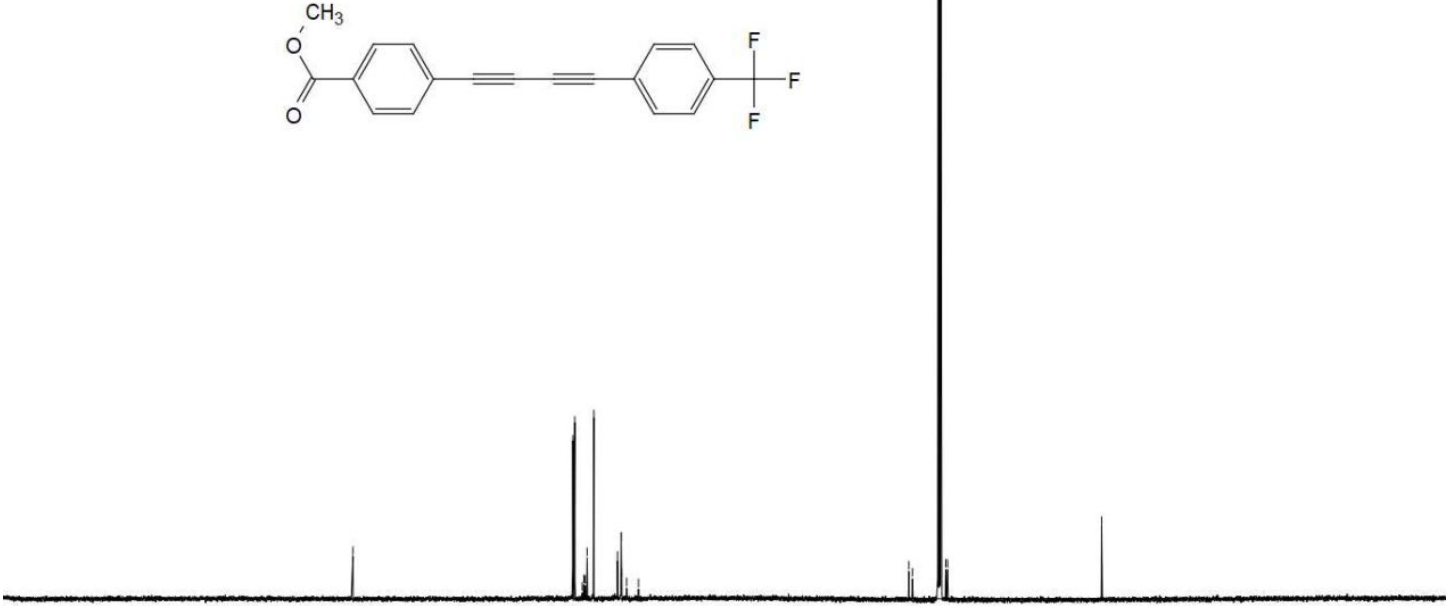

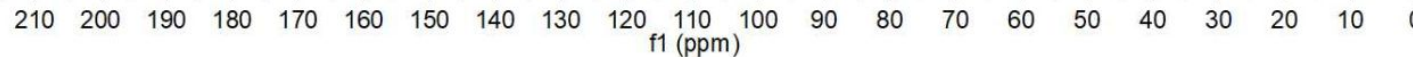

Figure 90. ${ }^{13} \mathrm{C}$ NMR spectroscopy of $4 \mathrm{~m}$. 


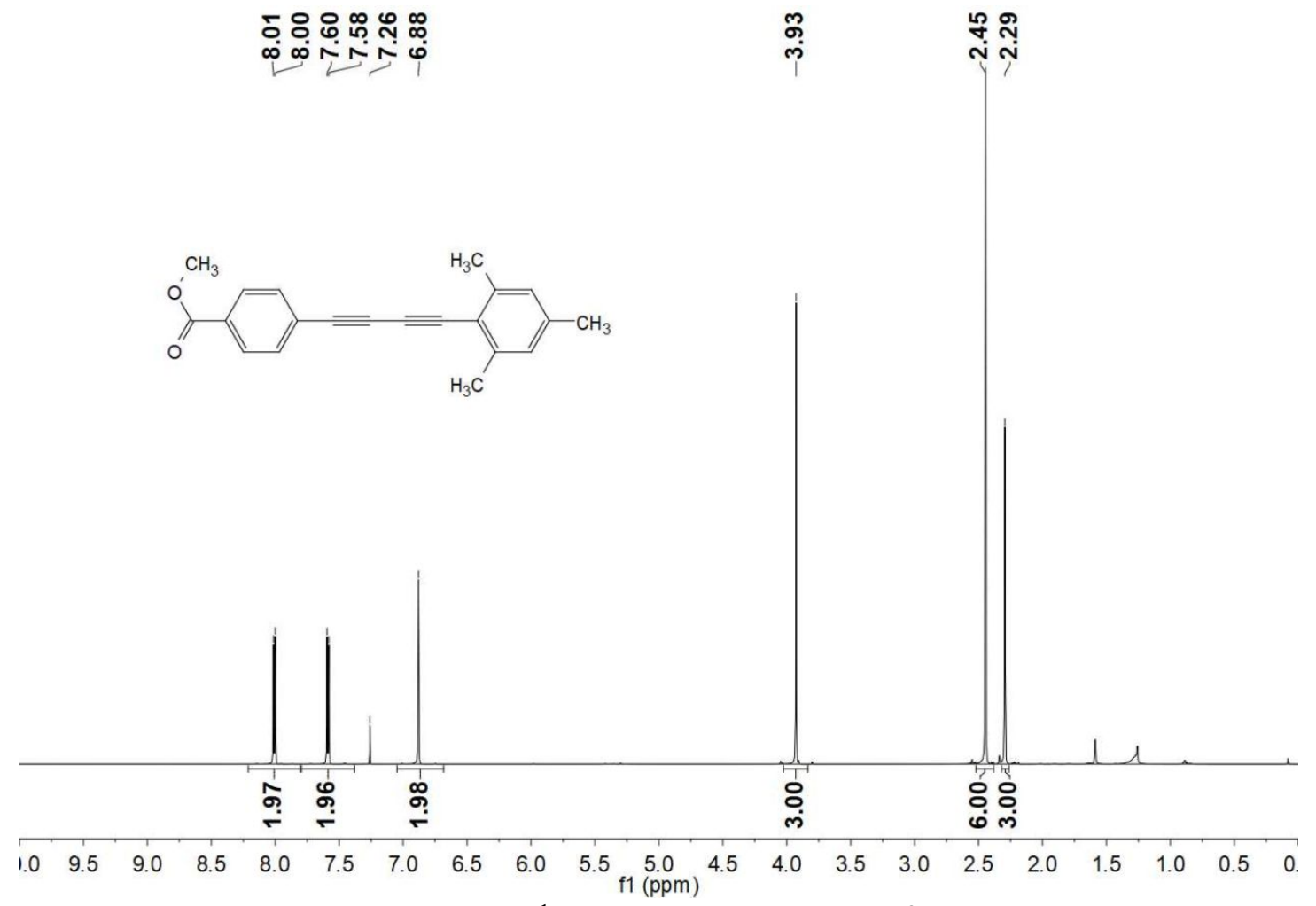

Figure S91. ${ }^{1} \mathrm{H}$ NMR spectroscopy of $\mathbf{4 n}$.

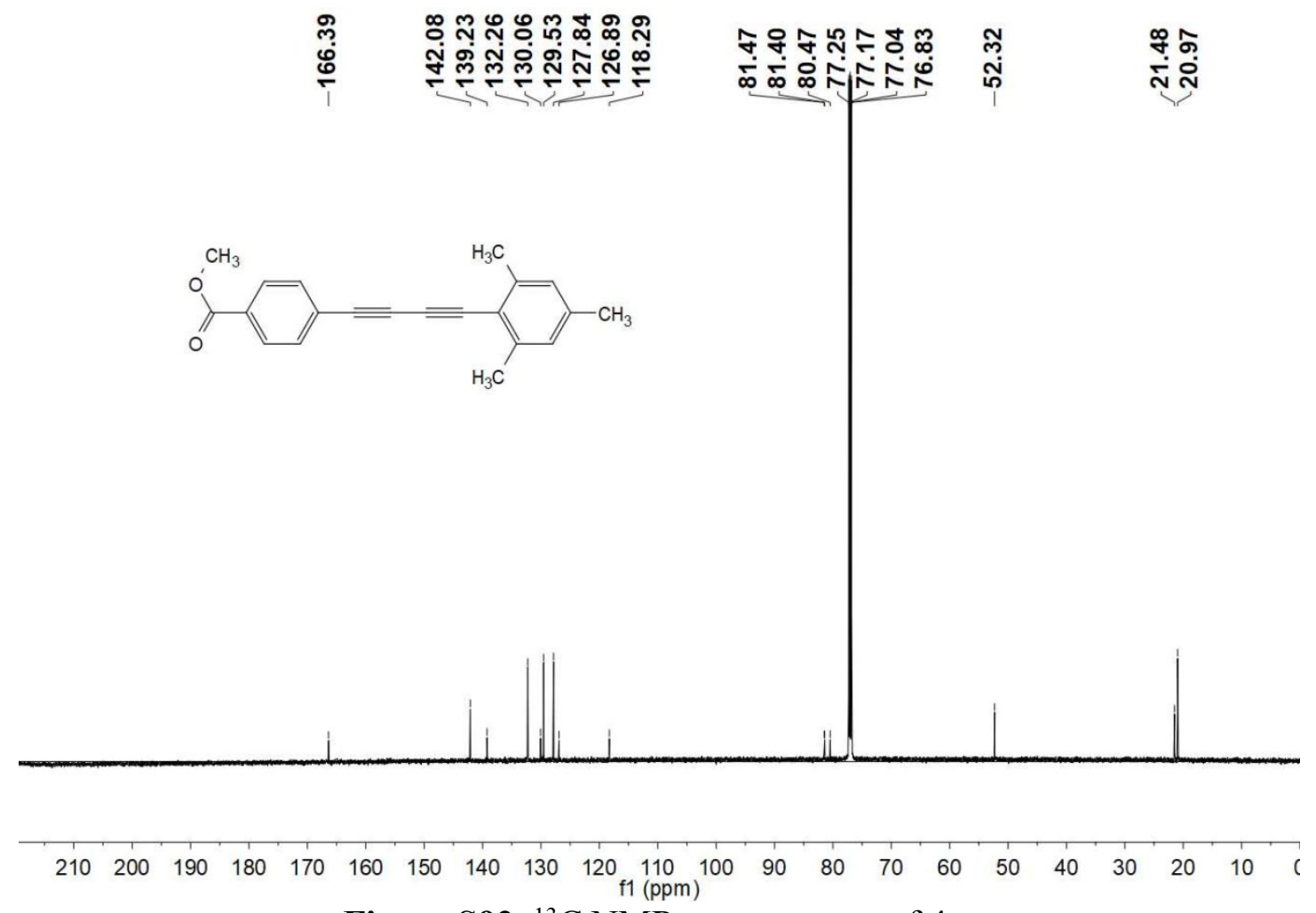

Figure S92. ${ }^{13} \mathrm{C}$ NMR spectroscopy of $\mathbf{4 n}$. 

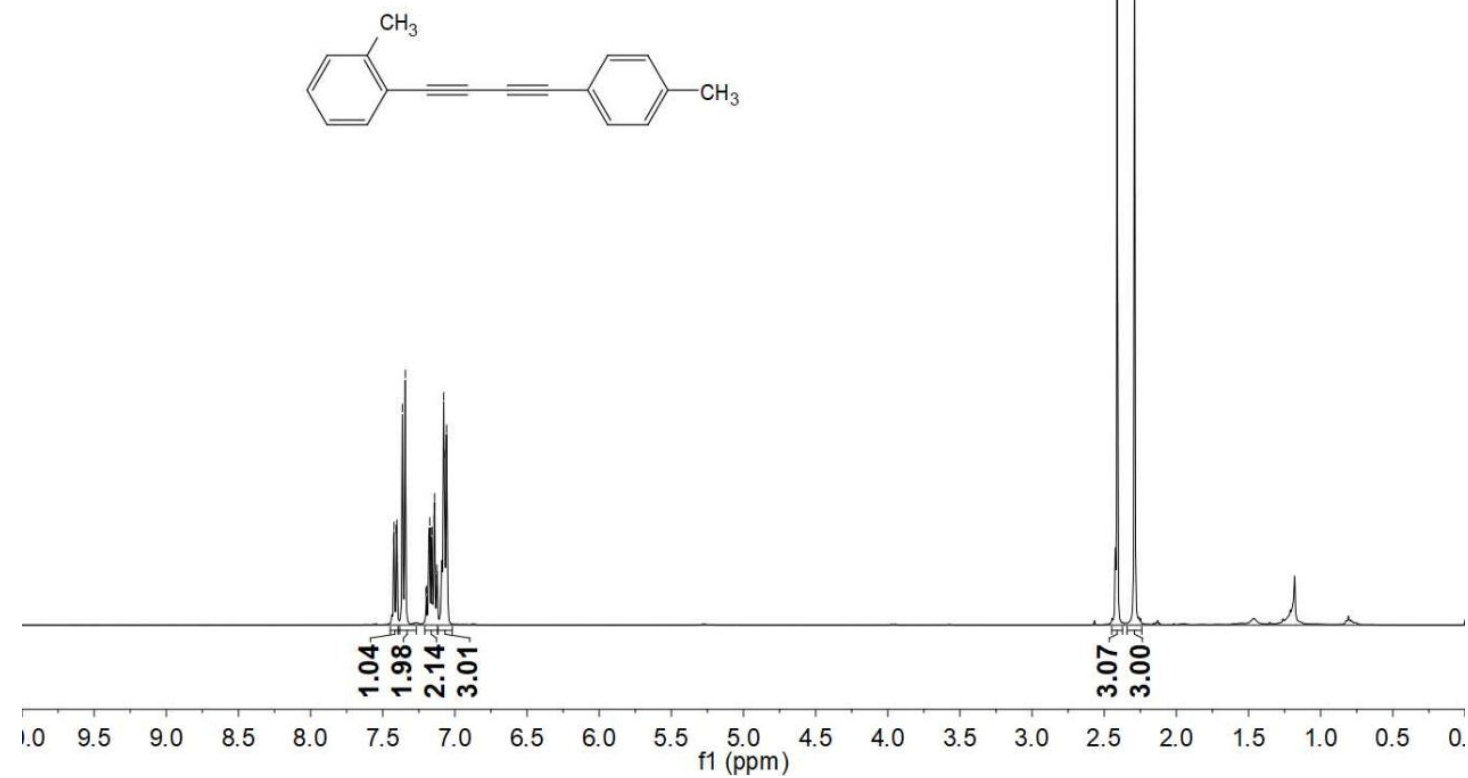

Figure S93. ${ }^{1} \mathrm{H}$ NMR spectroscopy of 40.

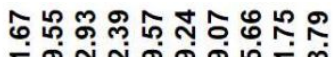

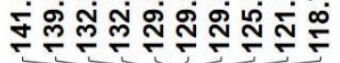

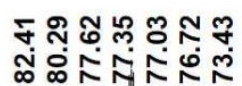

ยฺุ

సั่
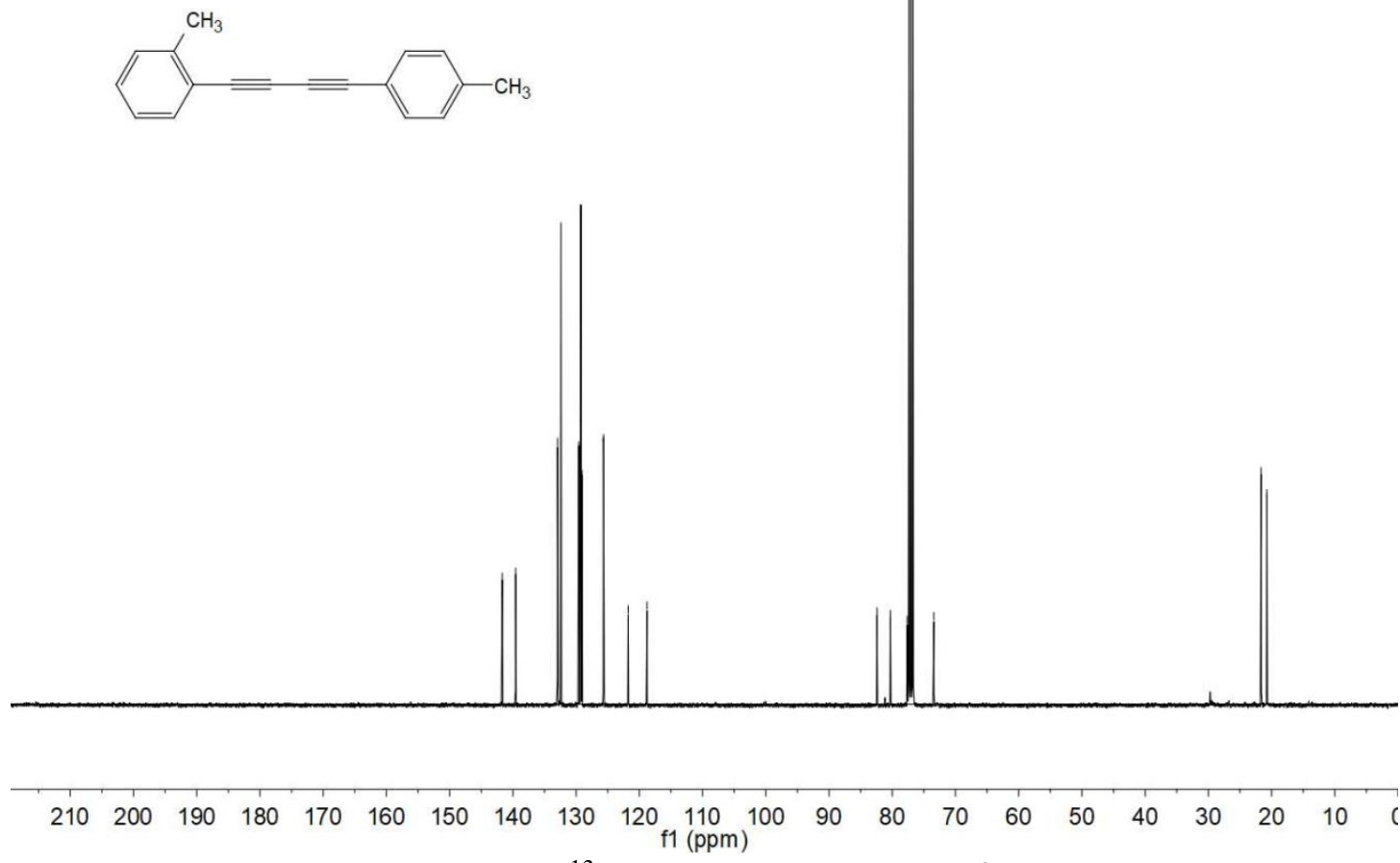

Figure S94. ${ }^{13} \mathrm{C}$ NMR spectroscopy of 40. 


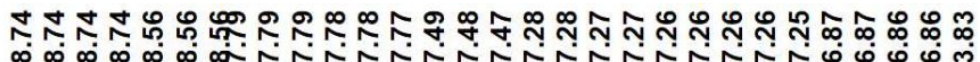

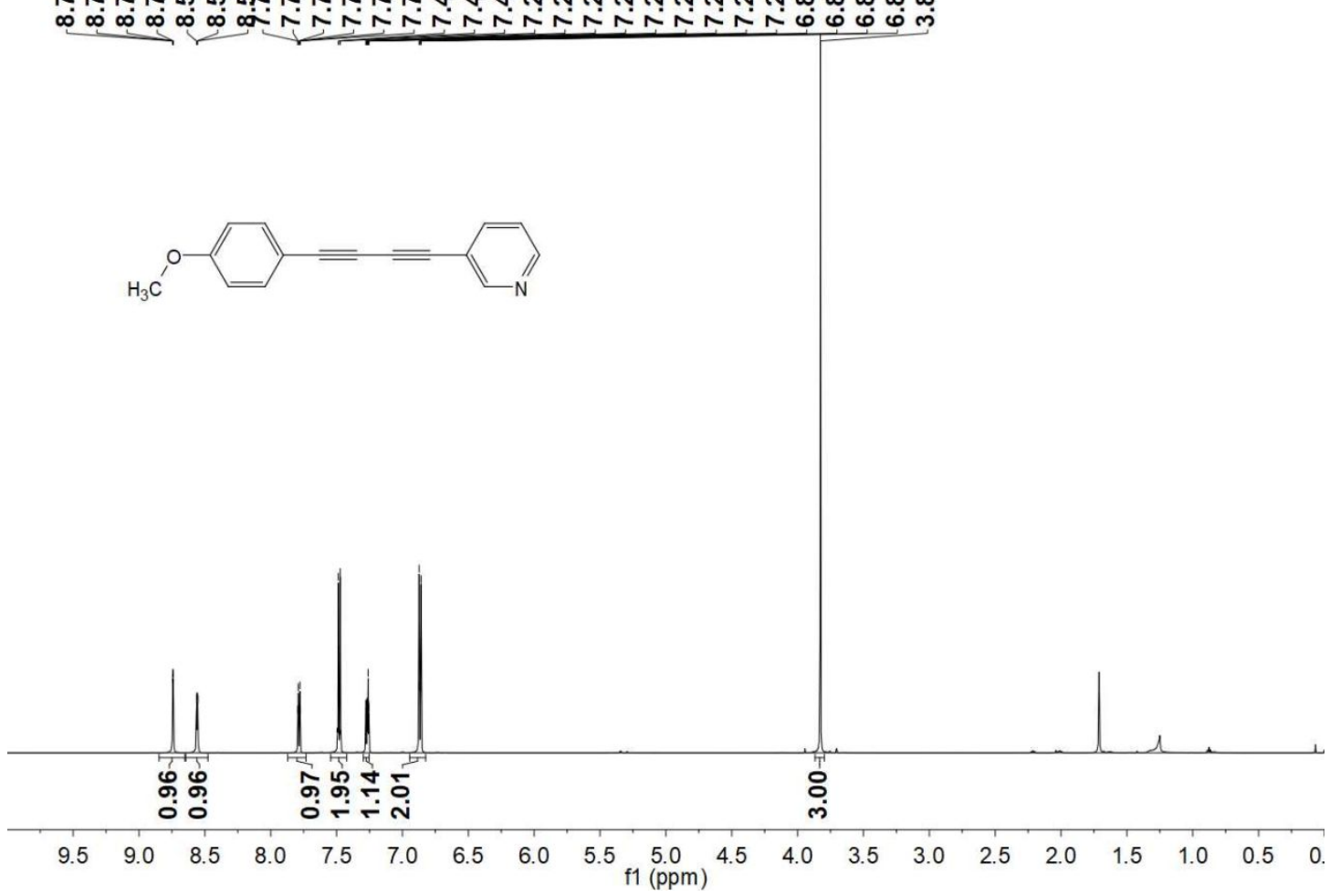

Figure S95. ${ }^{1} \mathrm{H}$ NMR spectroscopy of $\mathbf{4 p}$.

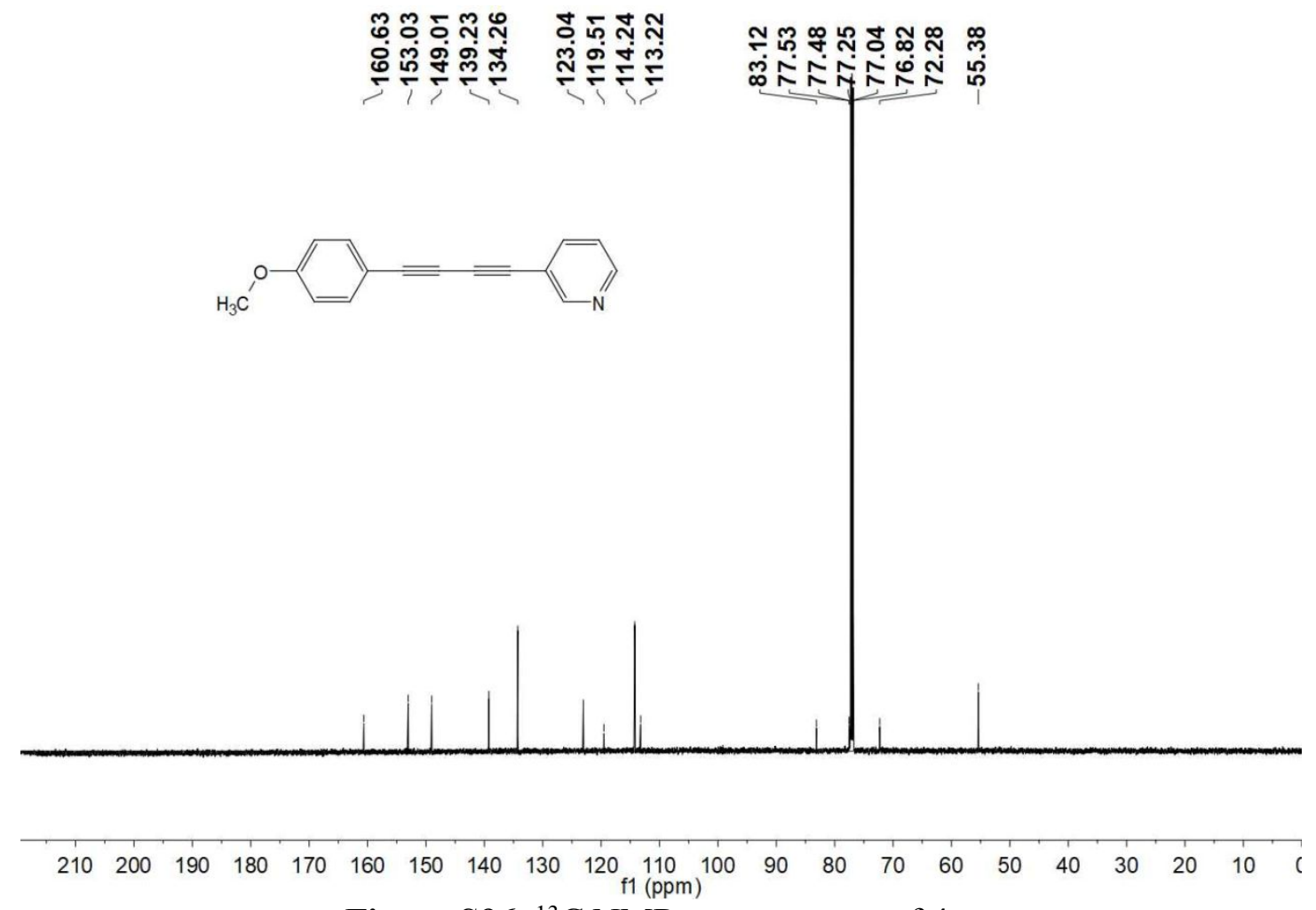

Figure S96. ${ }^{13} \mathrm{C}$ NMR spectroscopy of $4 p$. 


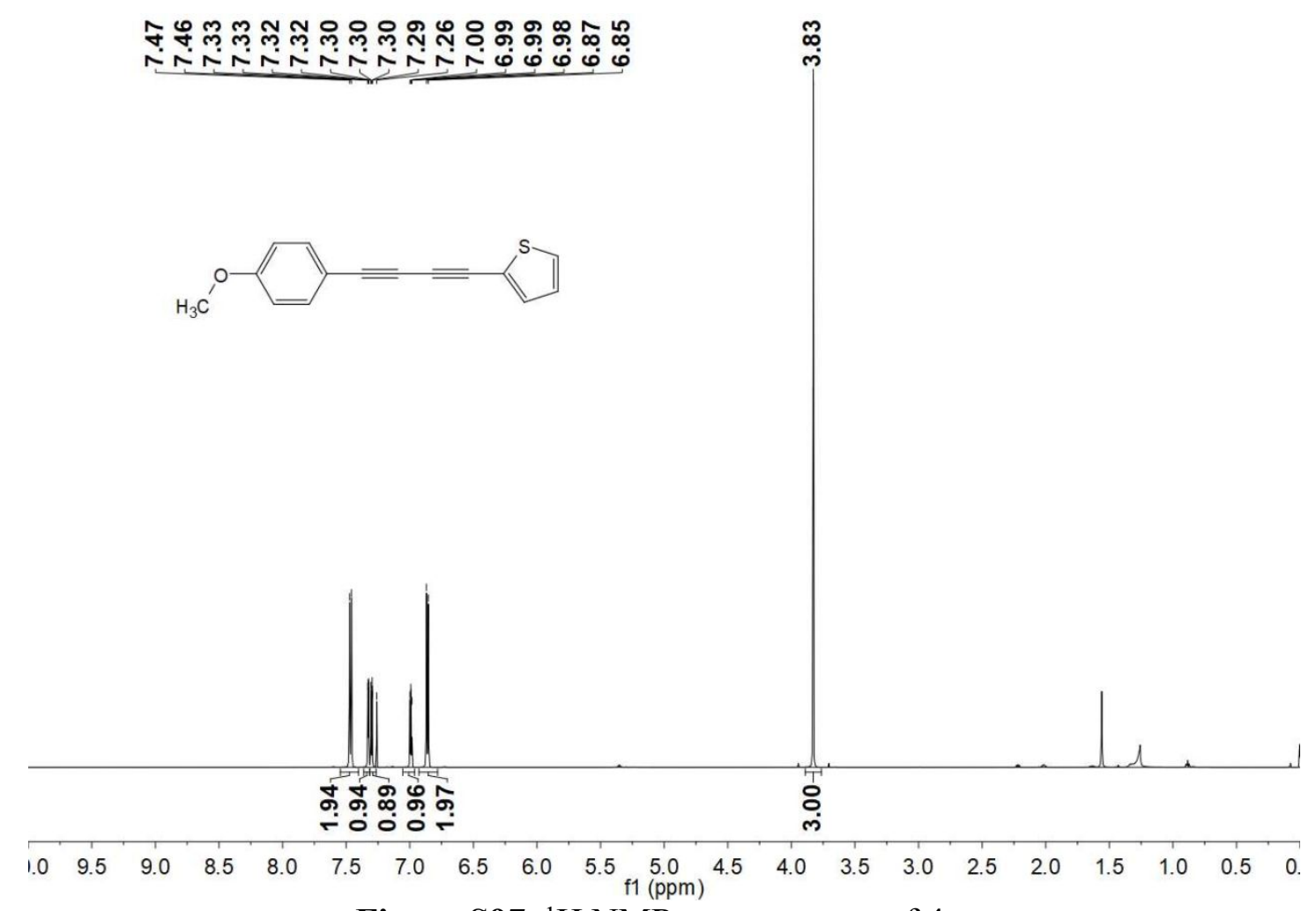

Figure S97. ${ }^{1} \mathrm{H}$ NMR spectroscopy of $\mathbf{4 q}$.

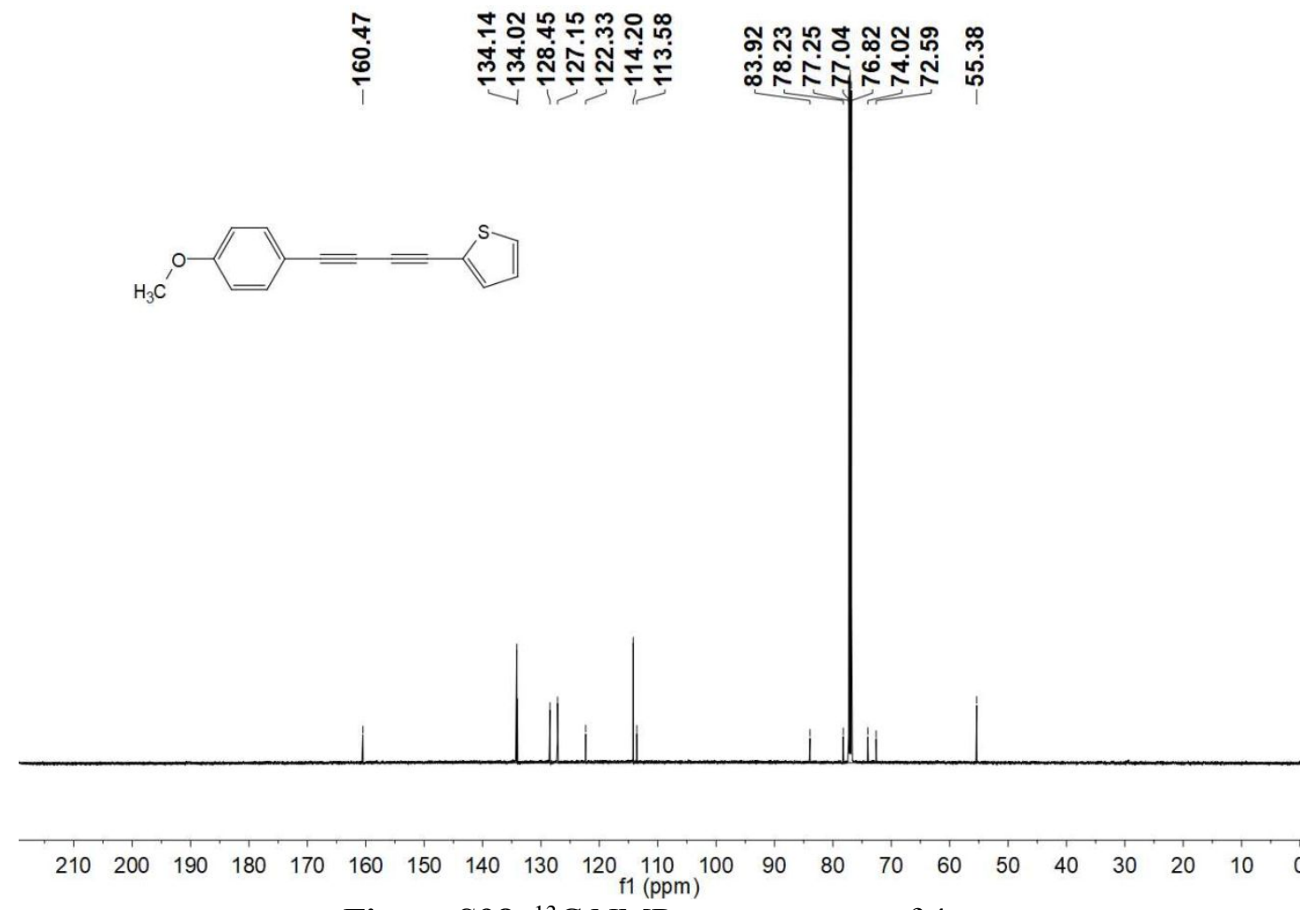

Figure S98. ${ }^{13} \mathrm{C}$ NMR spectroscopy of $\mathbf{4 q}$. 


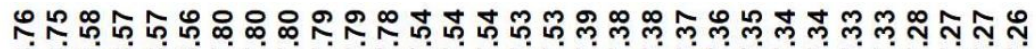

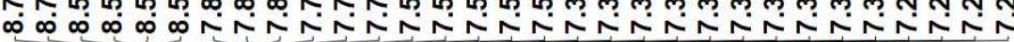

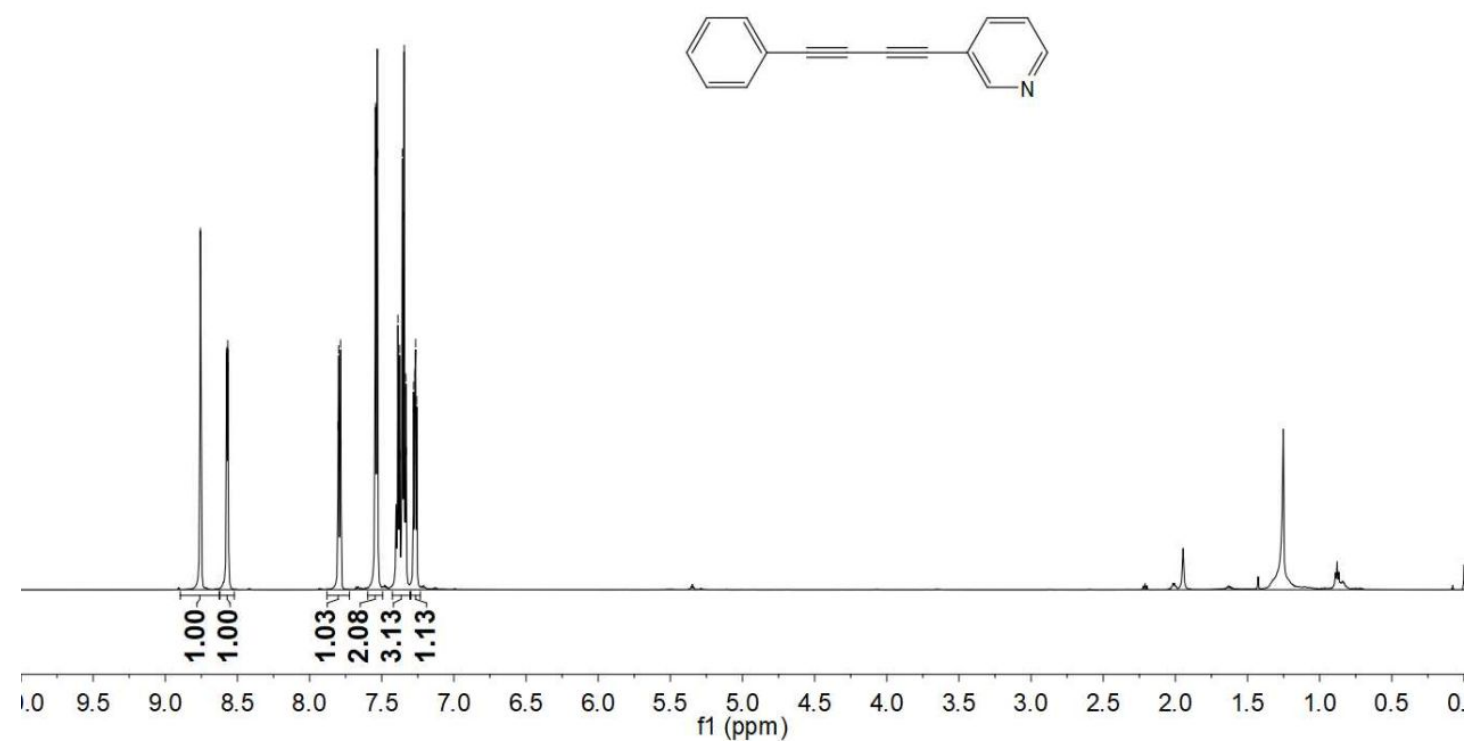

Figure S99. ${ }^{1} \mathrm{H}$ NMR spectroscopy of $4 \mathbf{r}$.

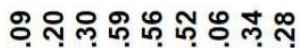

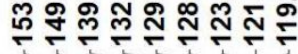

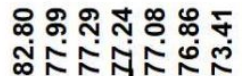
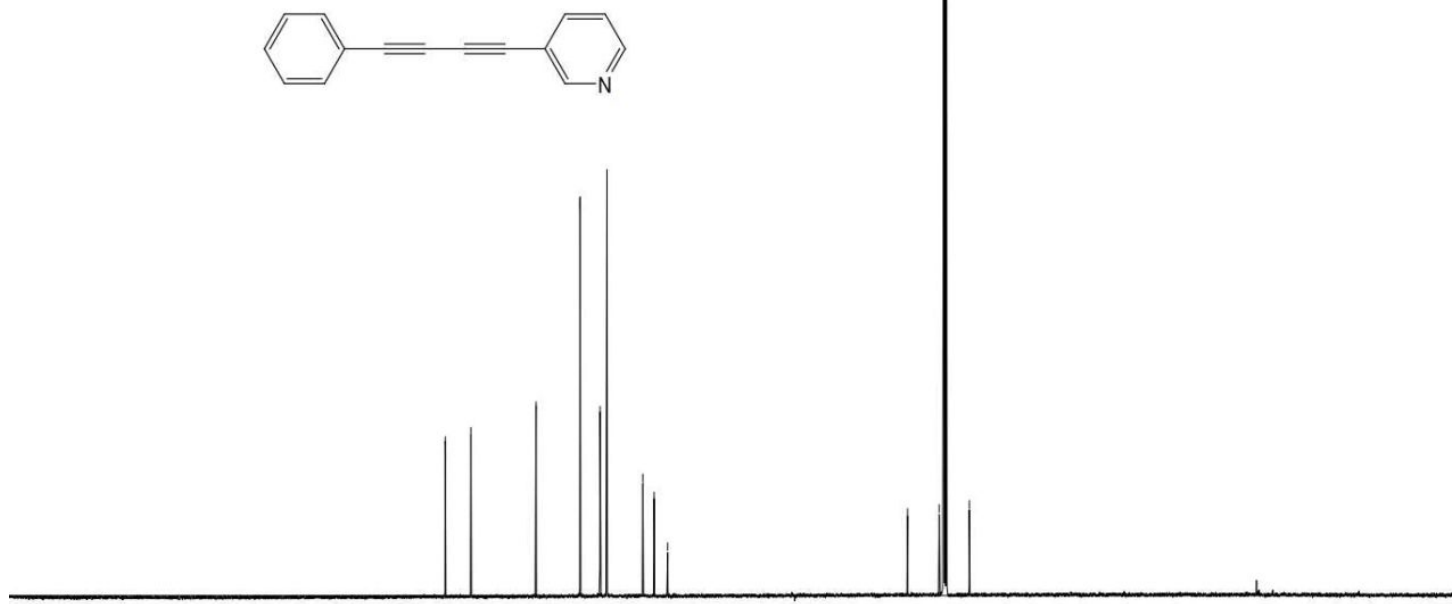

$\begin{array}{lllllllllllllllllllll}210 & 200 & 190 & 180 & 170 & 160 & 150 & 140 & 130 & 120 & \begin{array}{l}110 \\ 100\end{array} & 90 & 80 & 70 & 60 & 50 & 40 & 30 & 20 & 10\end{array}$

Figure S100. ${ }^{13} \mathrm{C}$ NMR spectroscopy of $4 r$. 

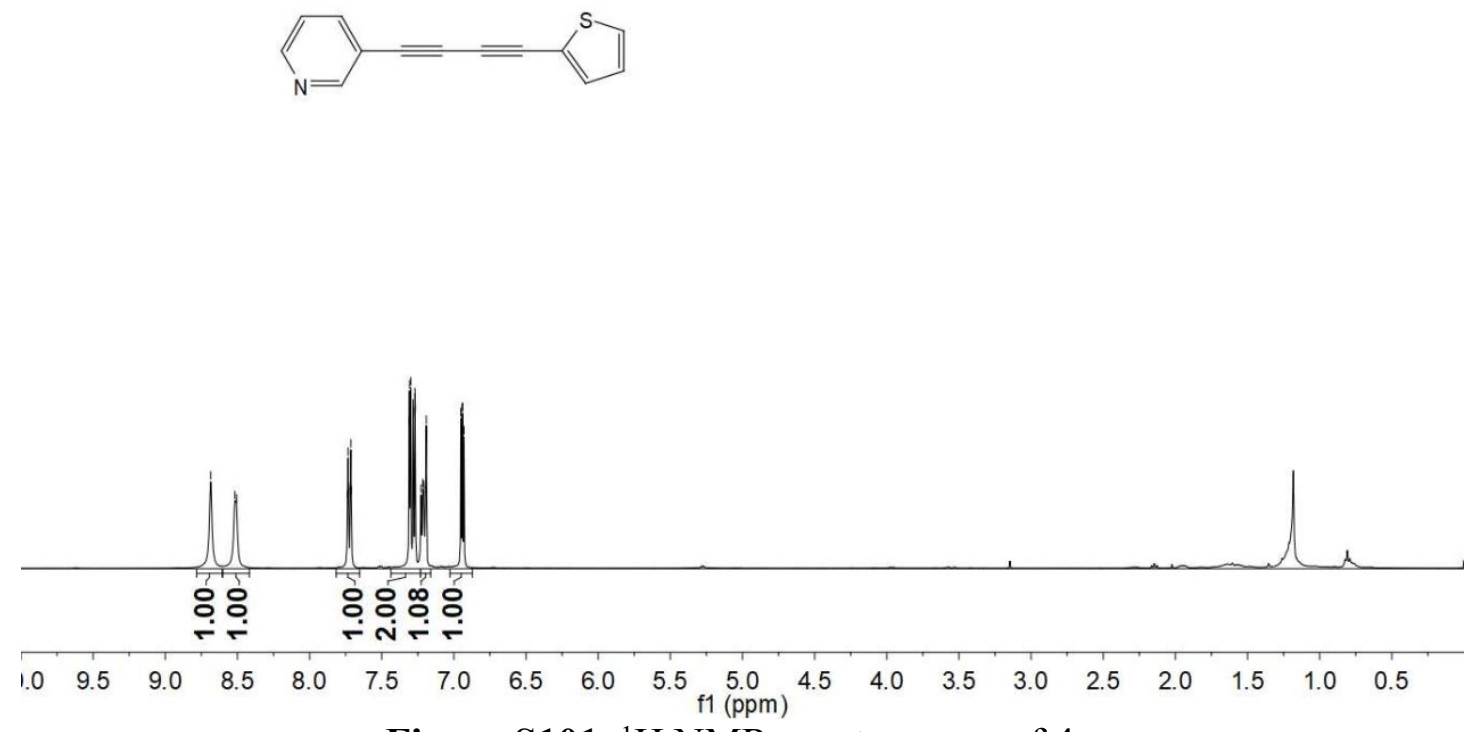

Figure S101. ${ }^{1} \mathrm{H}$ NMR spectroscopy of $4 \mathbf{s}$.

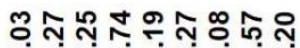

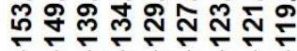

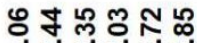

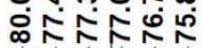
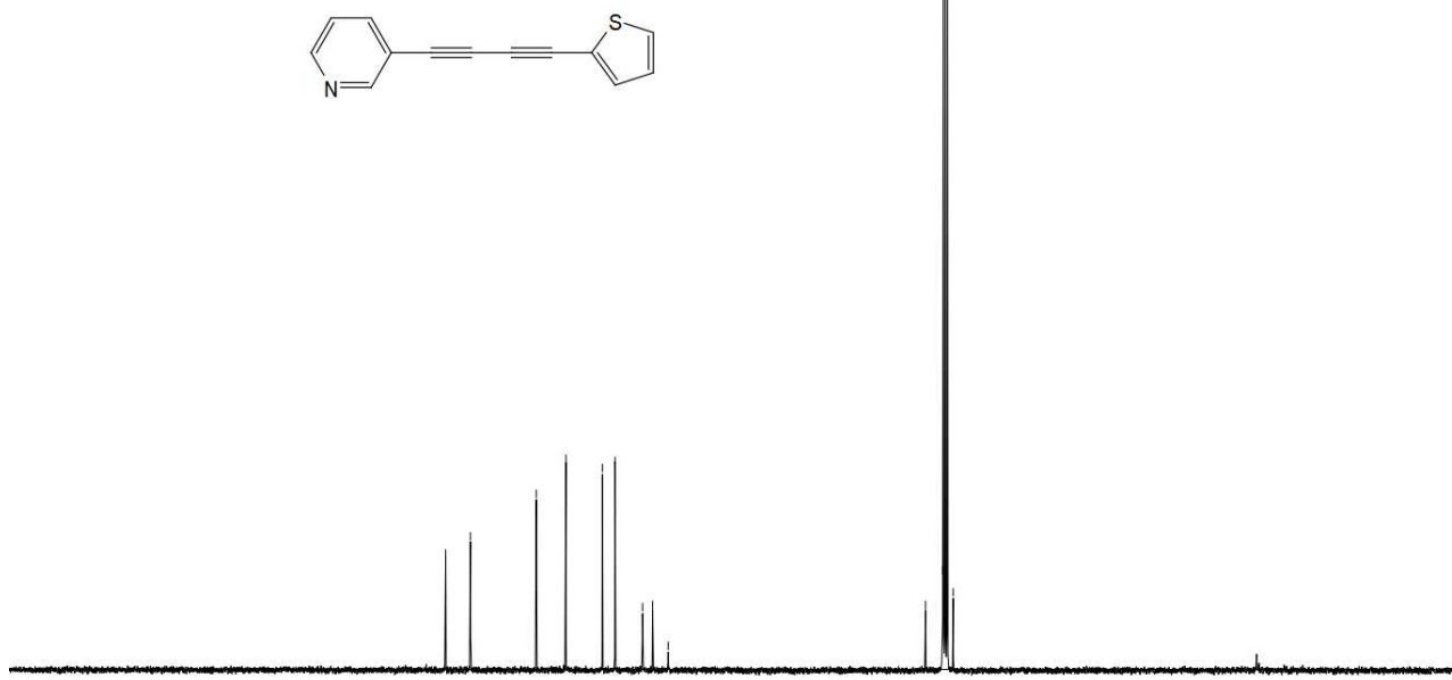

$\begin{array}{lllllllllllllllllllll}210 & 200 & 190 & 180 & 170 & 160 & 150 & 140 & 130 & 120 & 110 & 100 & 90 & 80 & 70 & 60 & 50 & 40 & 30 & 20 & 10\end{array}$

Figure S102. ${ }^{13} \mathrm{C}$ NMR spectroscopy of $4 \mathrm{~s}$. 


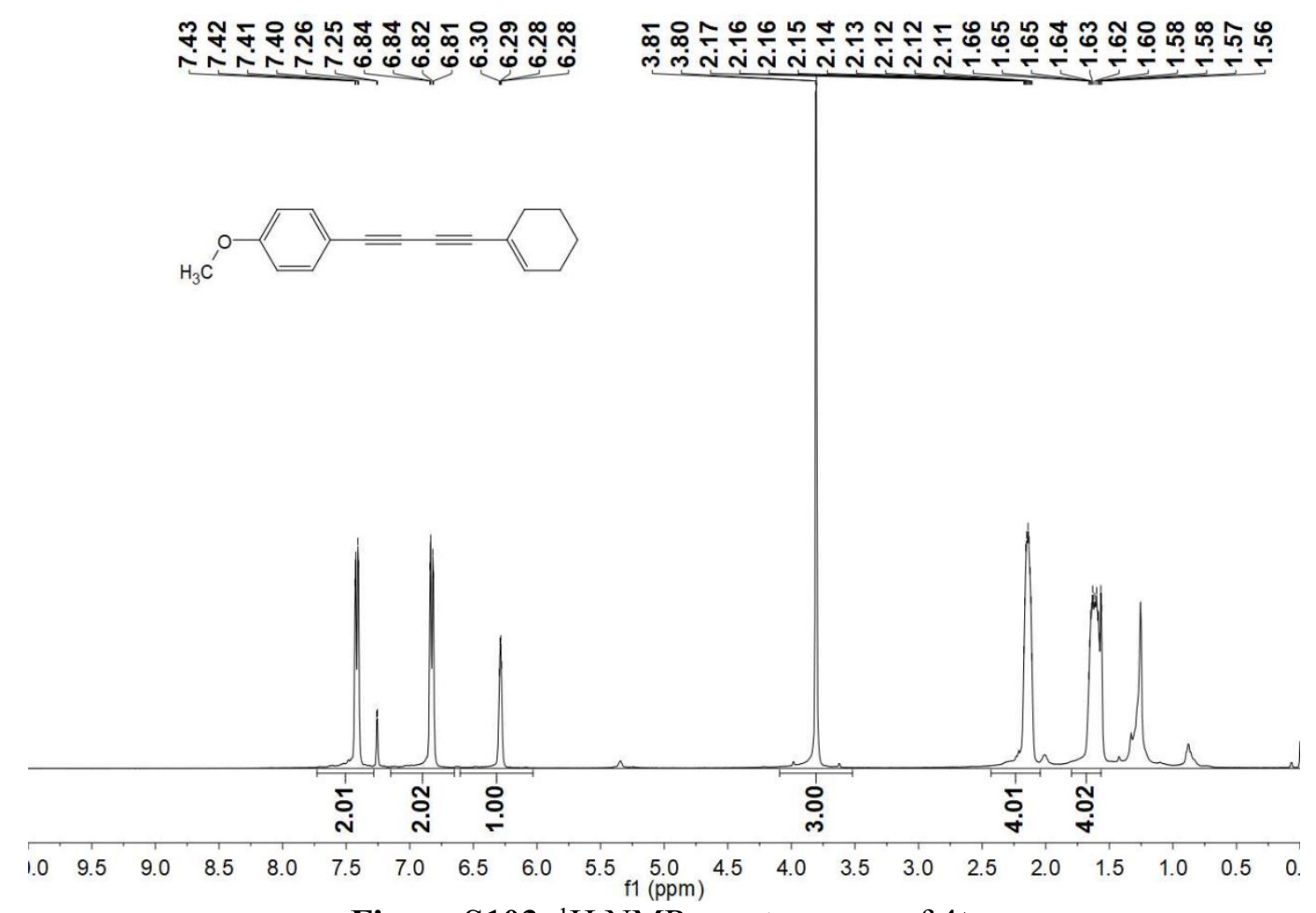

Figure S103. ${ }^{1} \mathrm{H}$ NMR spectroscopy of $\mathbf{4 t}$.

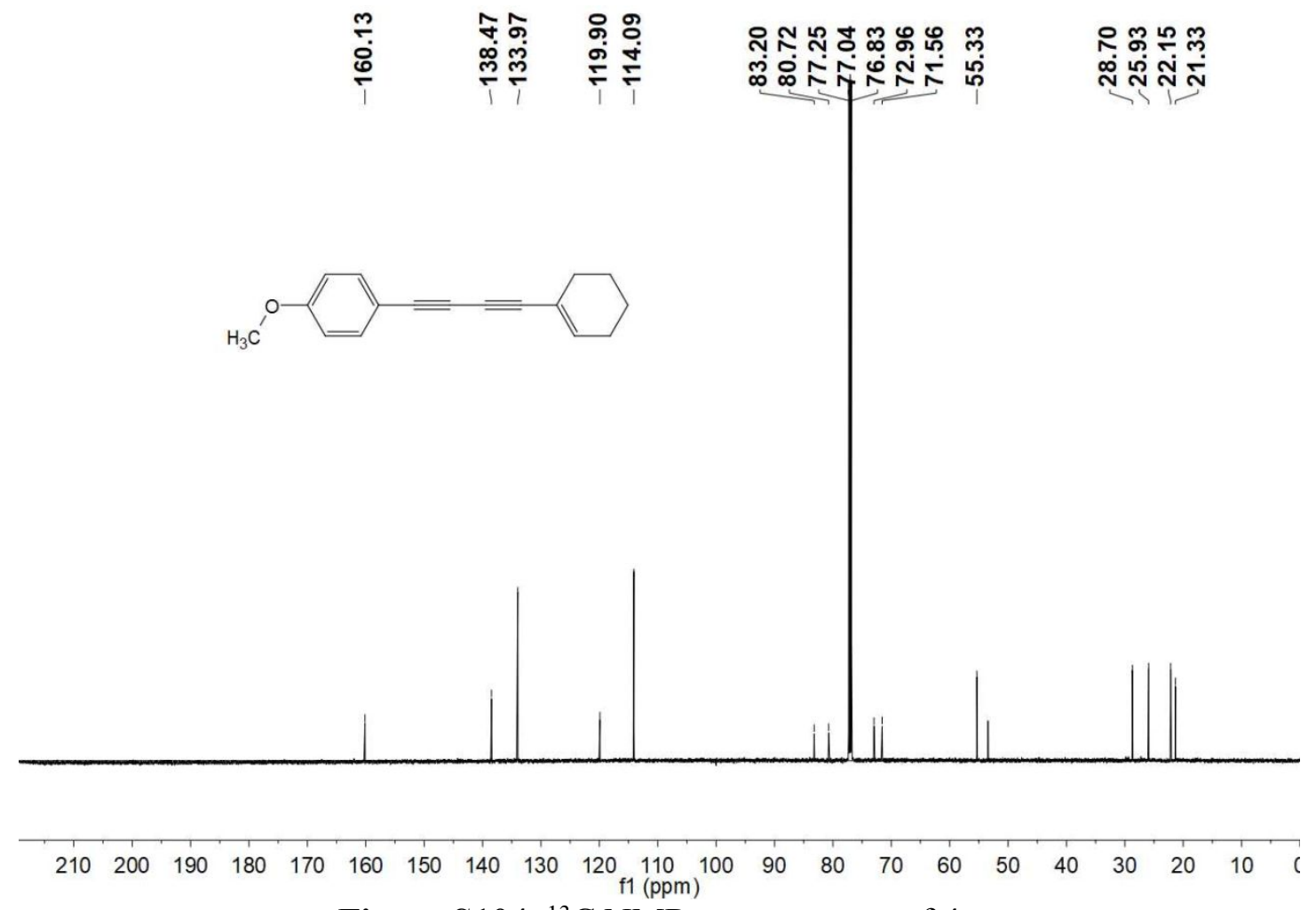

Figure S104. ${ }^{13} \mathrm{C}$ NMR spectroscopy of $\mathbf{4 t}$. 


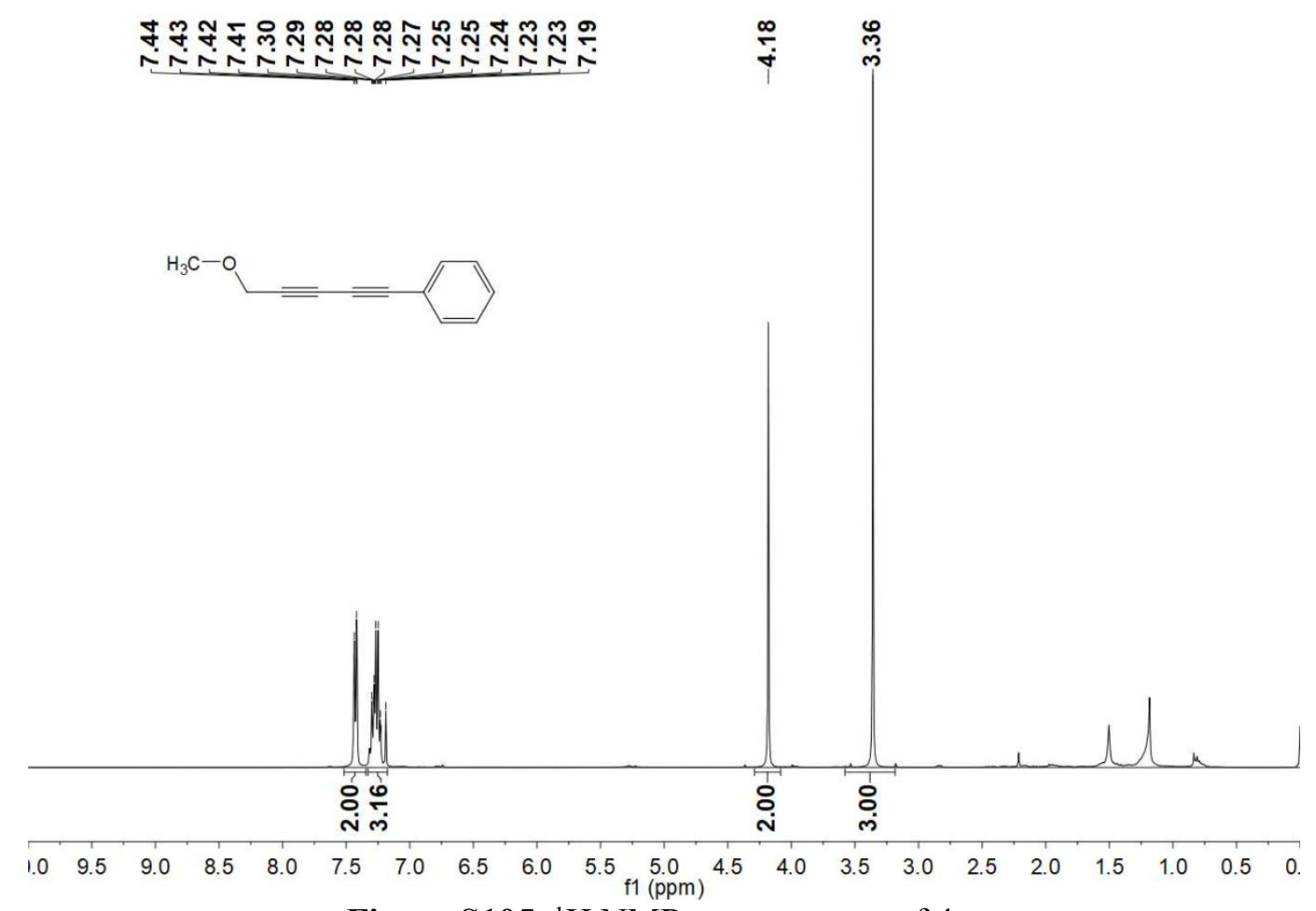

Figure S105. ${ }^{1} \mathrm{H}$ NMR spectroscopy of $\mathbf{4 u}$.

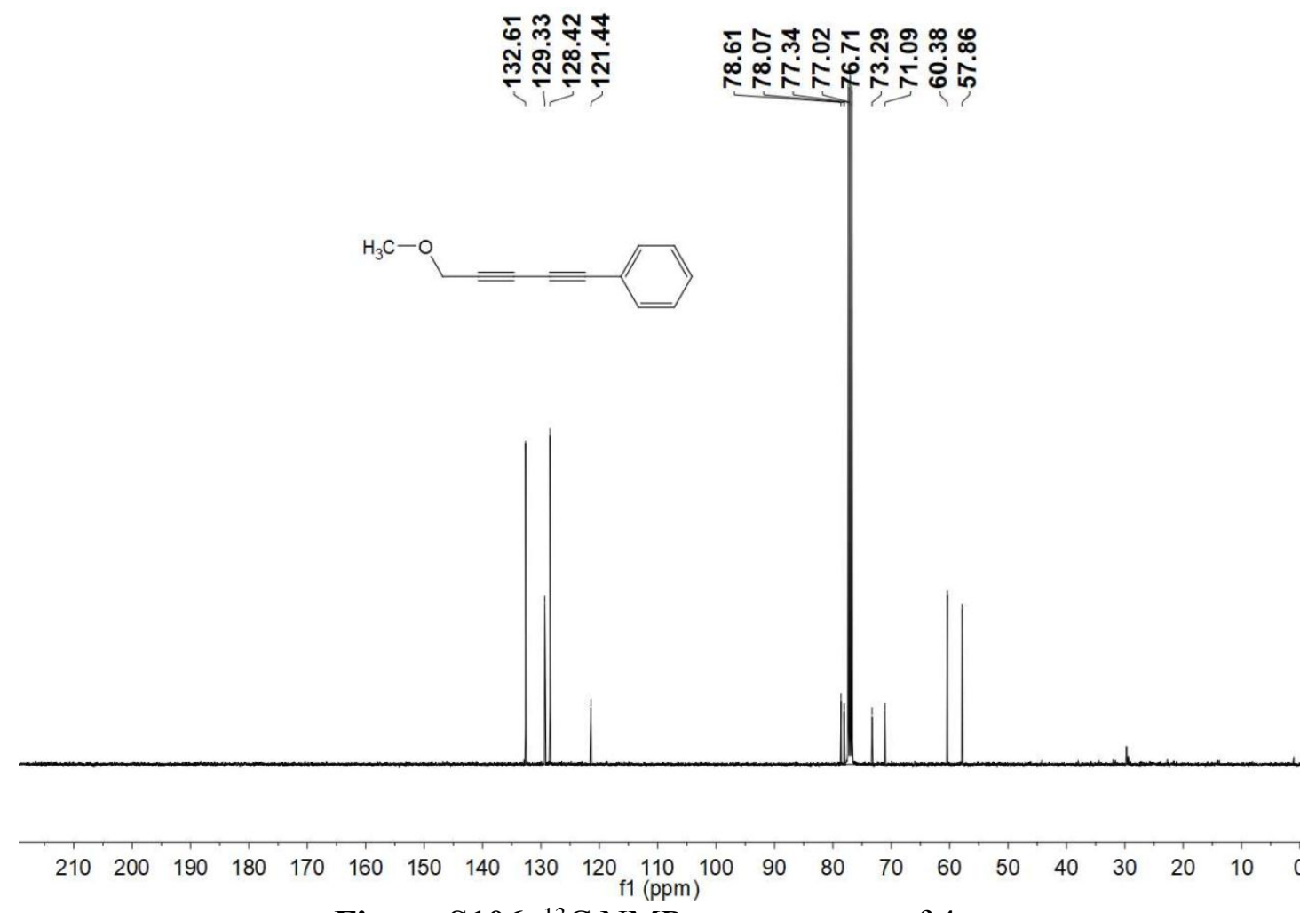

Figure S106. ${ }^{13} \mathrm{C}$ NMR spectroscopy of $\mathbf{4 u}$. 


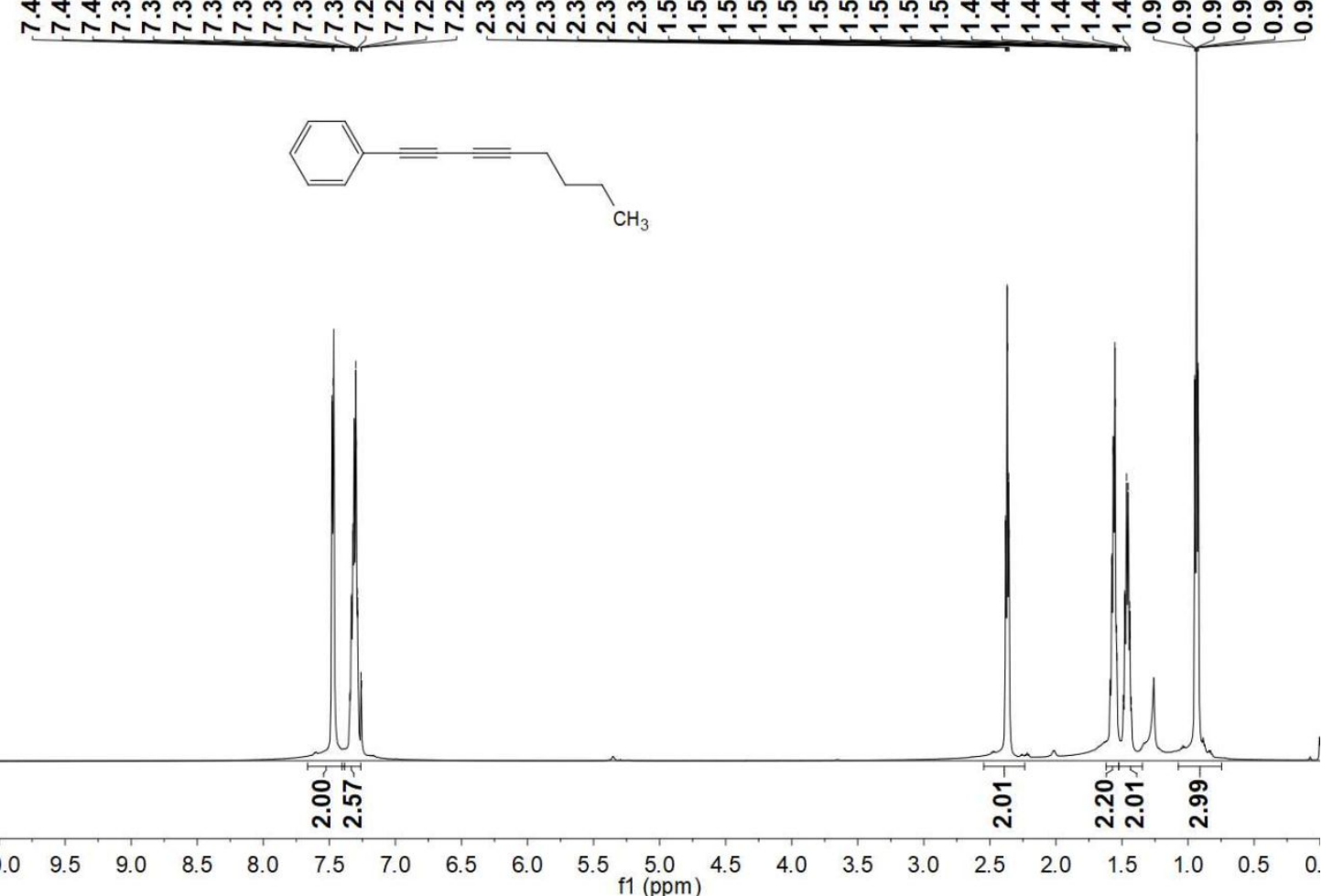

Figure S107. ${ }^{1} \mathrm{H}$ NMR spectroscopy of $4 \mathbf{v}$.

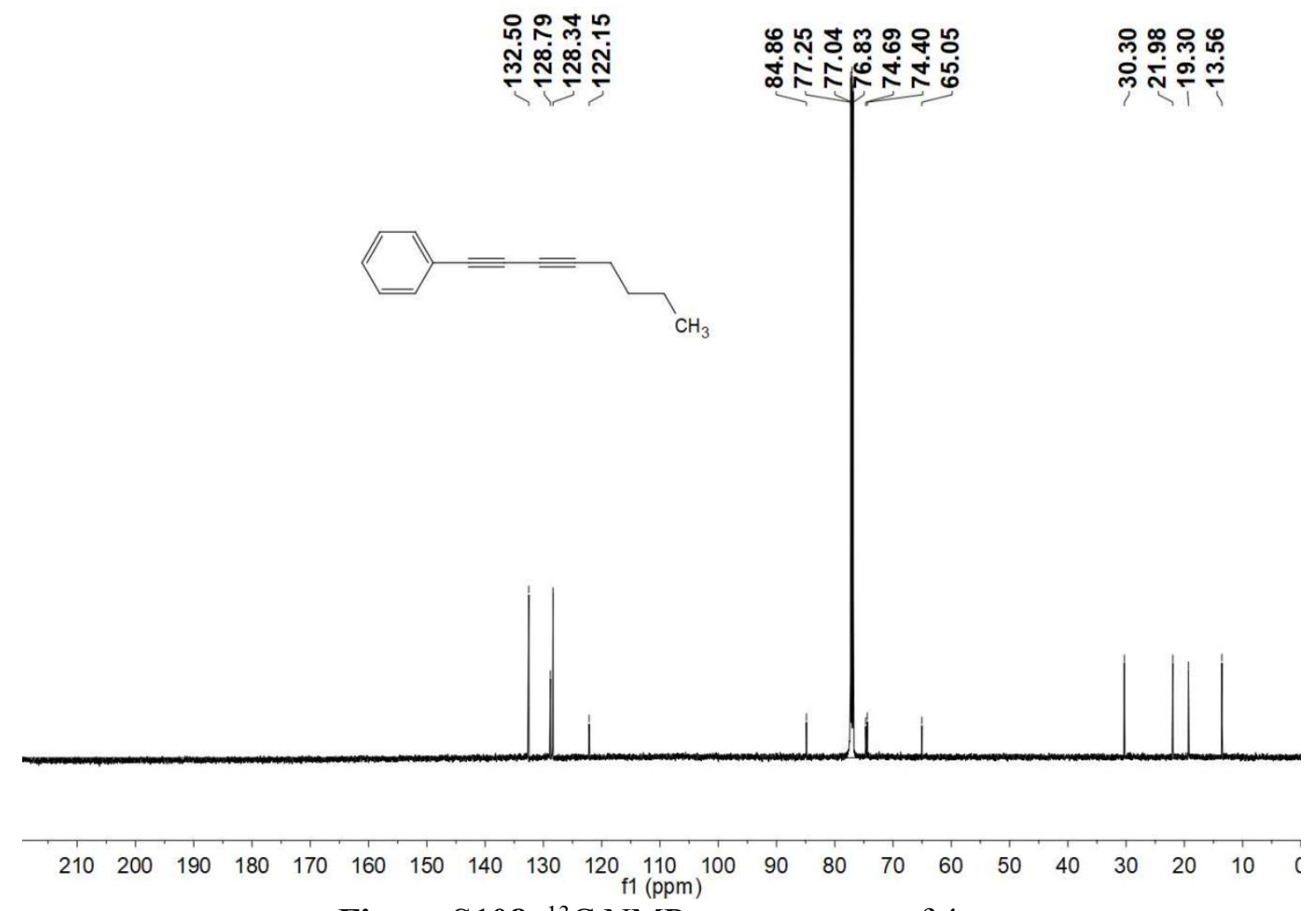

Figure S108. ${ }^{13} \mathrm{C}$ NMR spectroscopy of $4 \mathbf{v}$. 


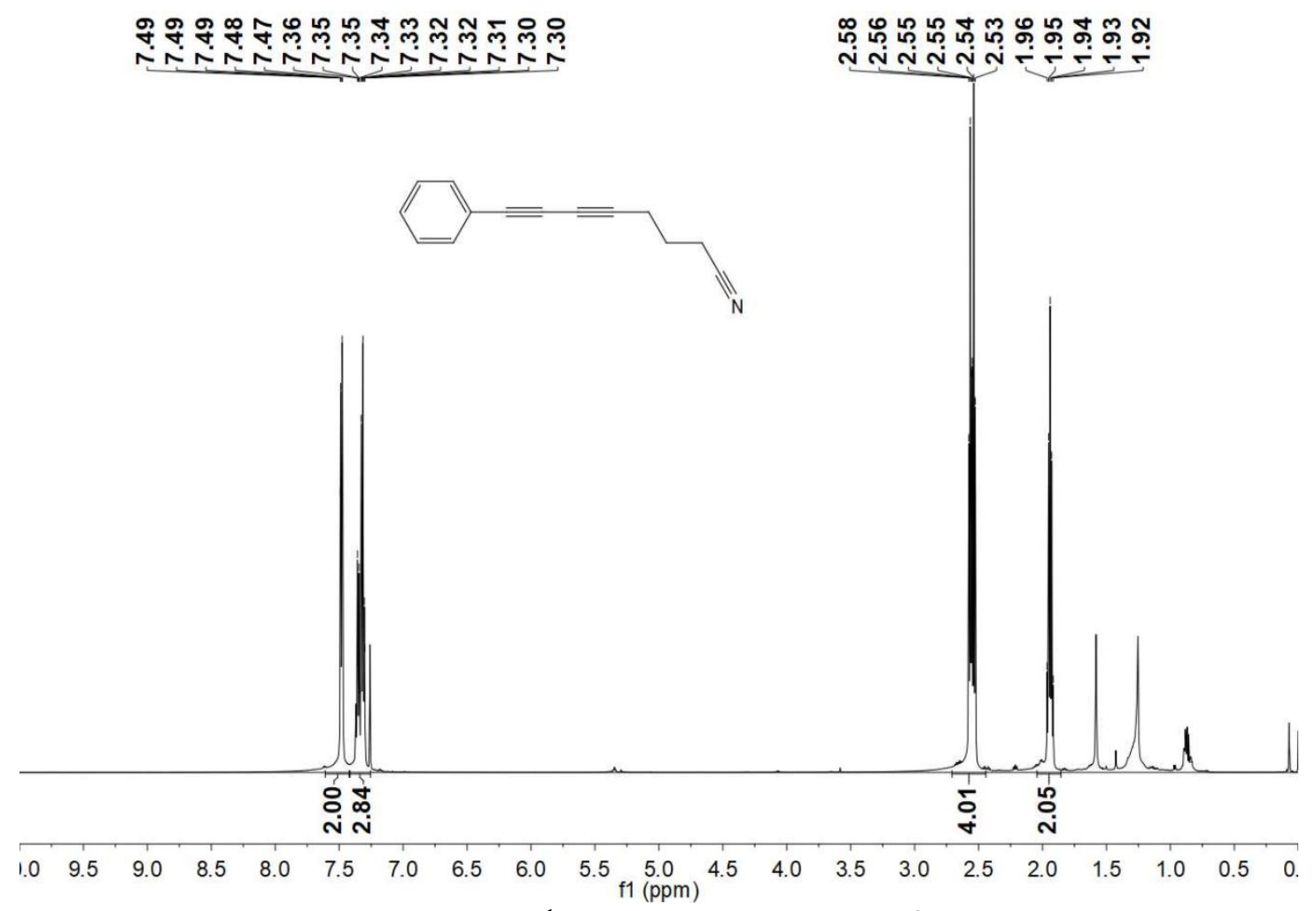

Figure S109. ${ }^{1} \mathrm{H}$ NMR spectroscopy of $4 \mathbf{w}$.

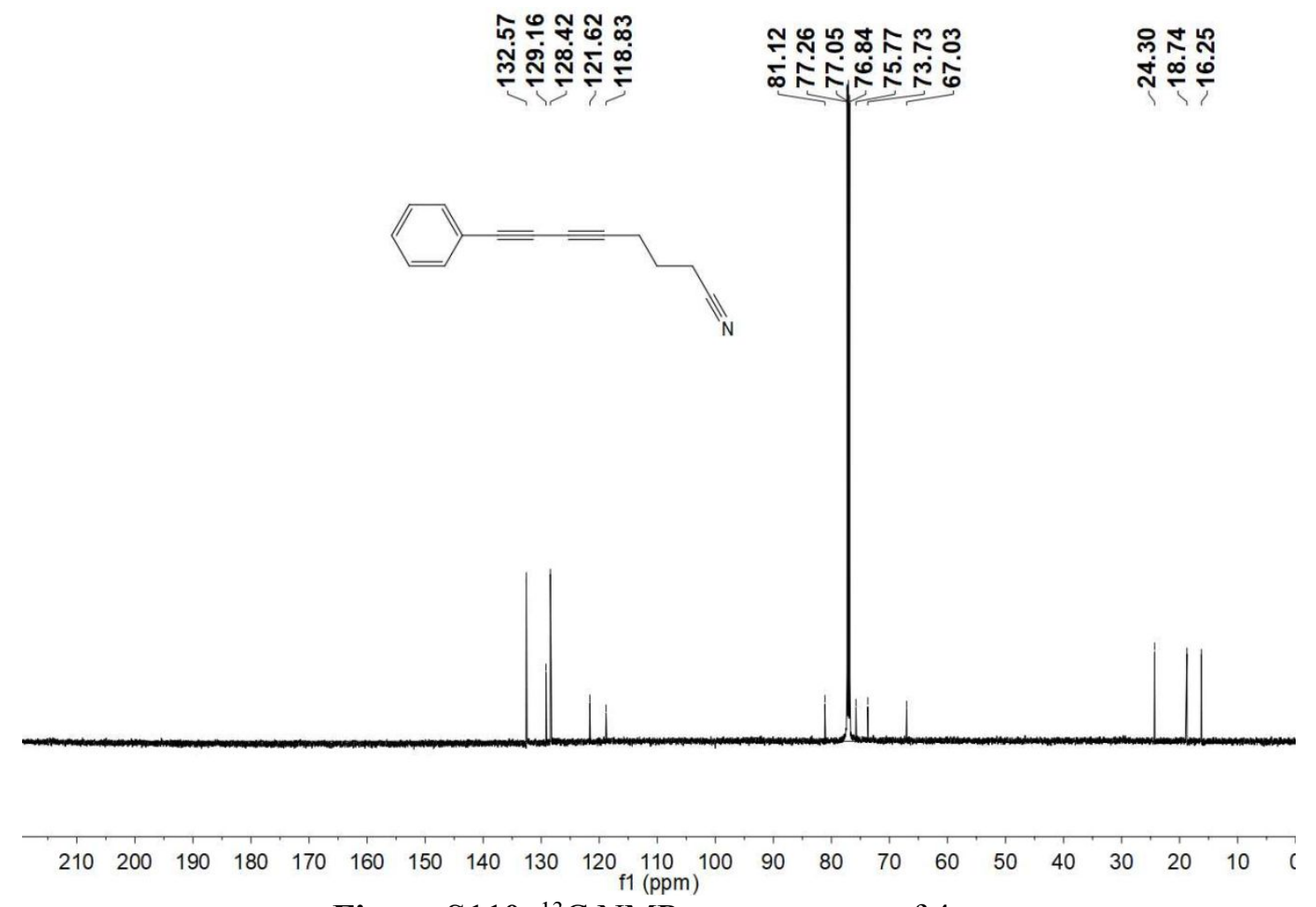

Figure S110. ${ }^{13} \mathrm{C}$ NMR spectroscopy of $\mathbf{4 w}$. 

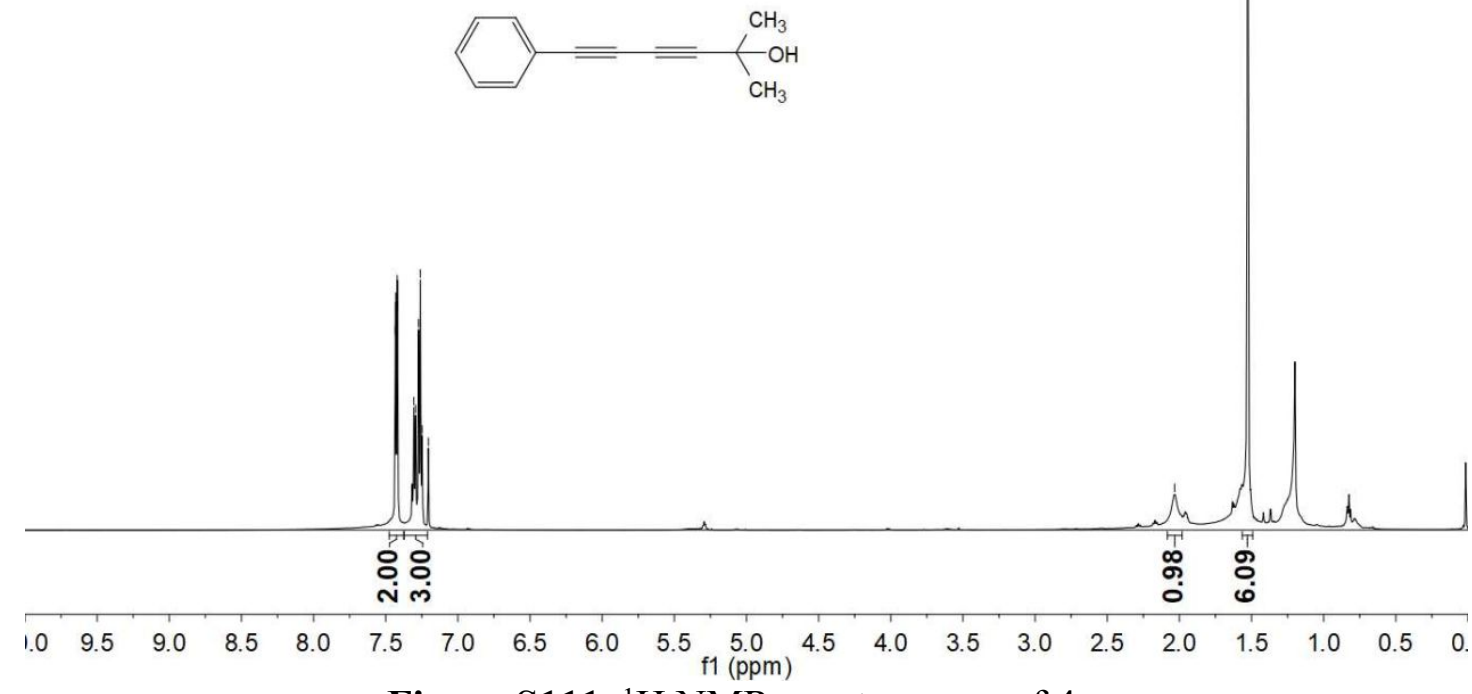

Figure S111. ${ }^{1} \mathrm{H}$ NMR spectroscopy of $\mathbf{4 x}$.

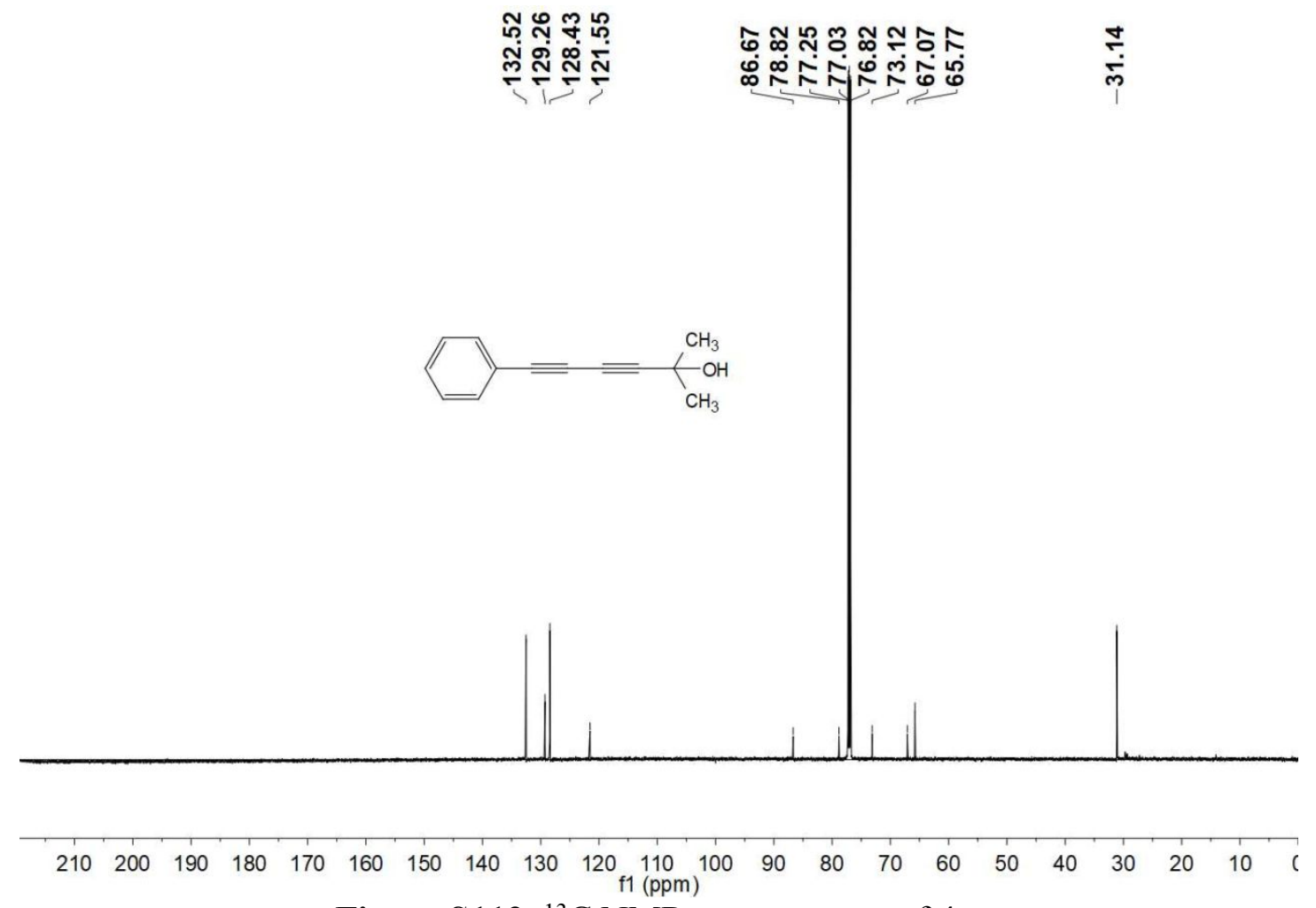

Figure S112. ${ }^{13} \mathrm{C}$ NMR spectroscopy of $4 \mathbf{x}$. 


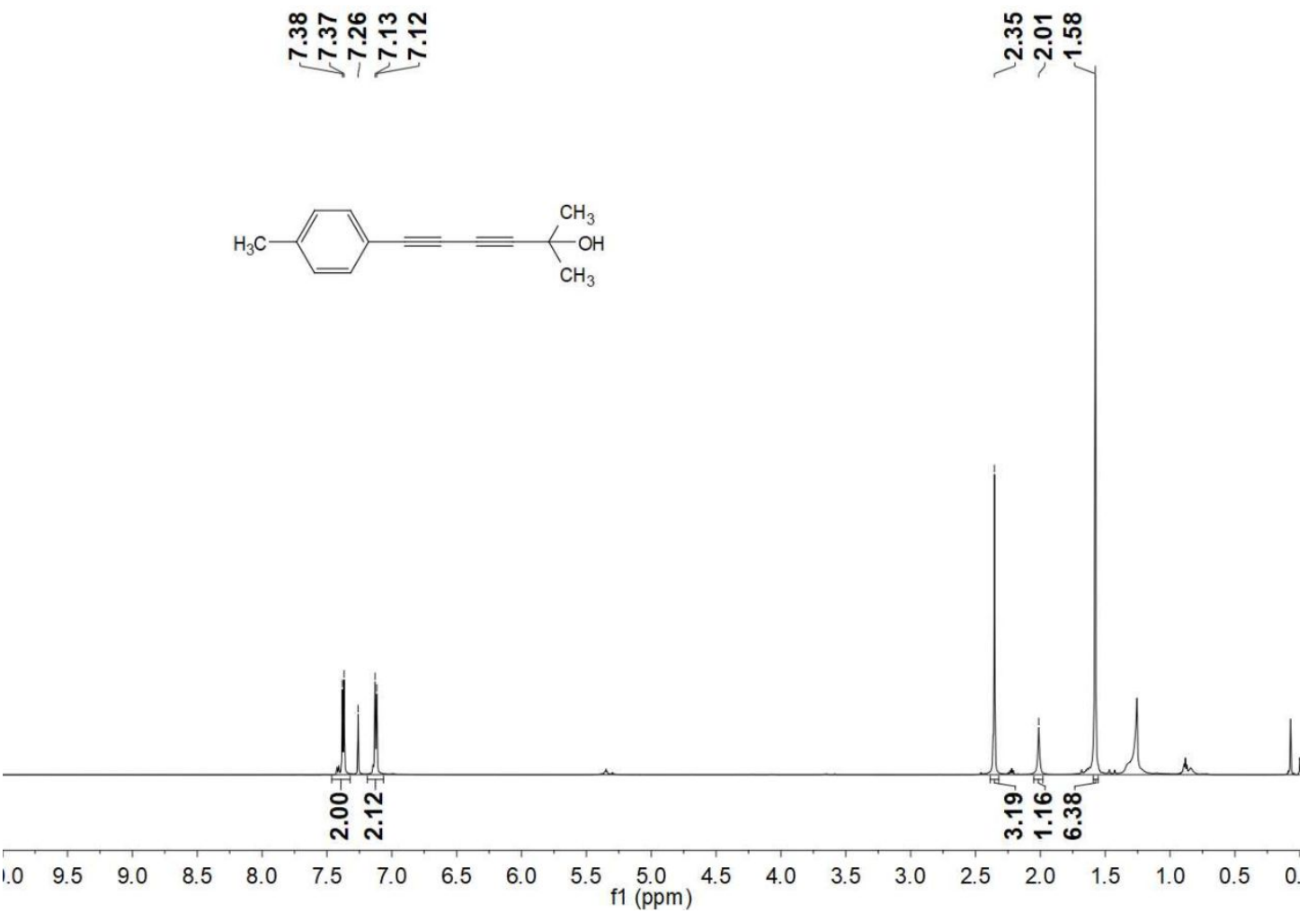

幽

Figure S113. ${ }^{1} \mathrm{H}$ NMR spectroscopy of $4 \mathbf{y}$.

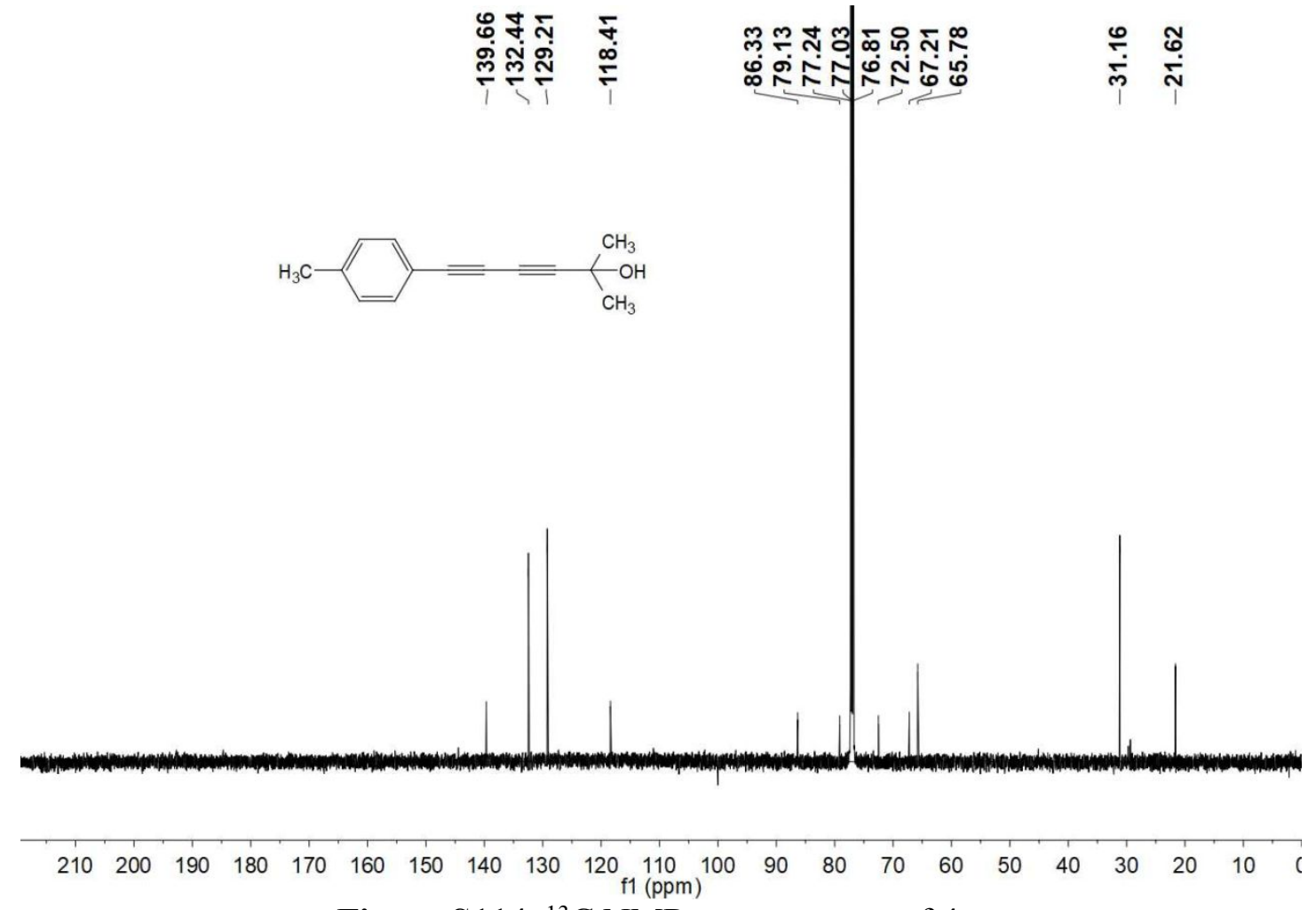

Figure S114. ${ }^{13} \mathrm{C}$ NMR spectroscopy of $4 y$. 


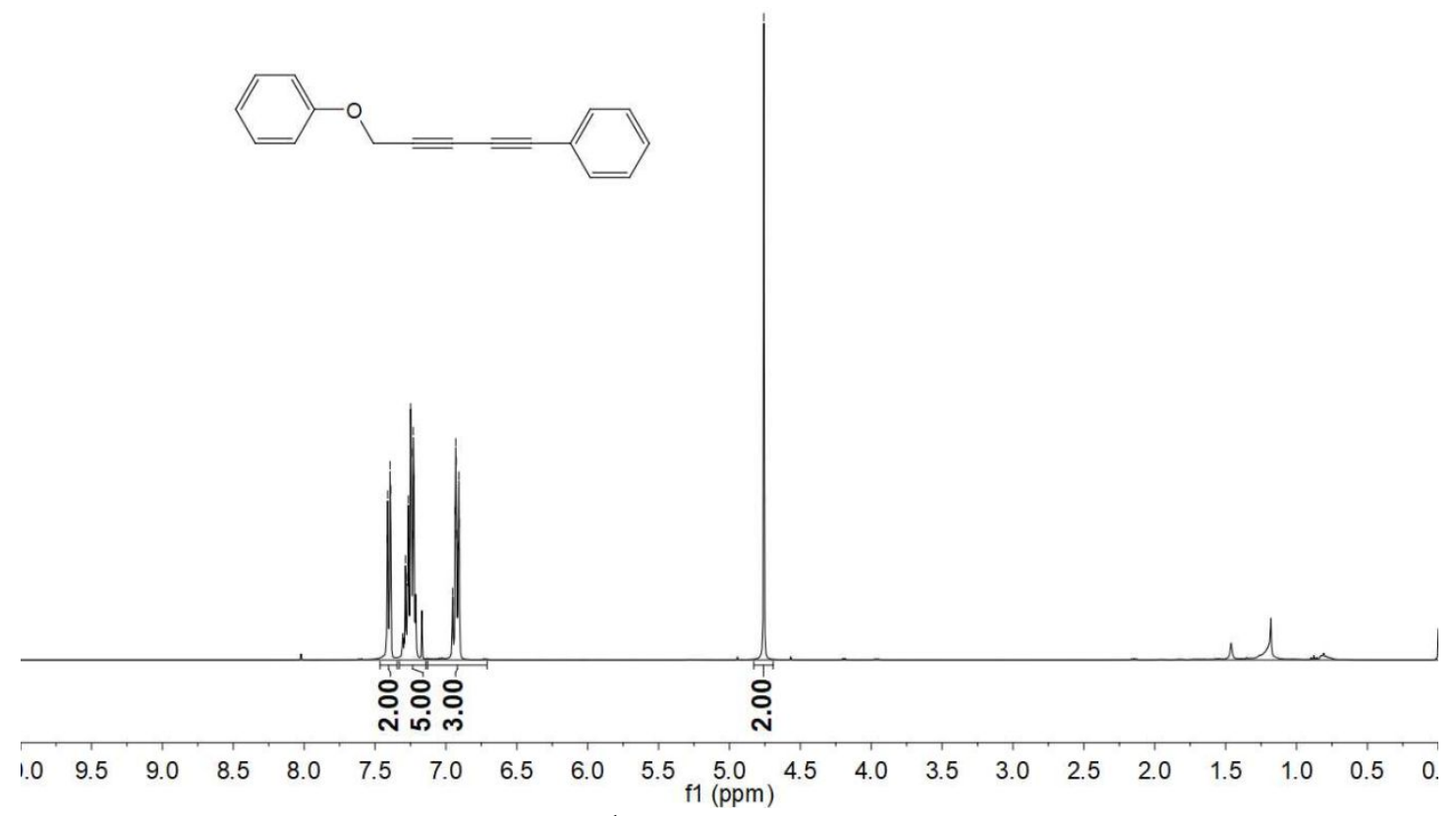

Figure S115. ${ }^{1} \mathrm{H}$ NMR spectroscopy of $\mathbf{4 z}$.

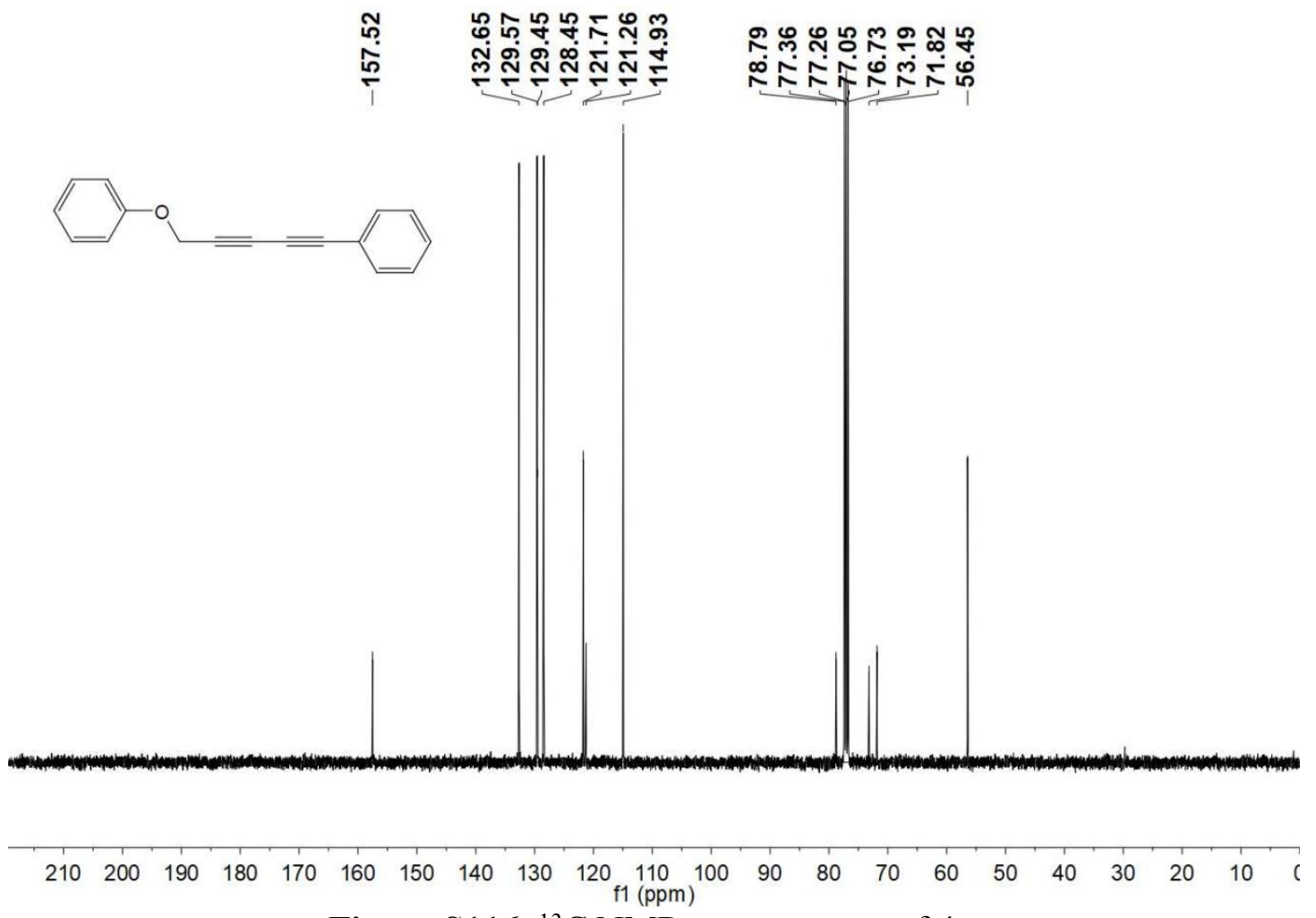

Figure S116. ${ }^{13} \mathrm{C}$ NMR spectroscopy of $4 z$. 


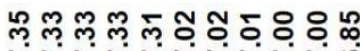

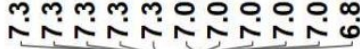

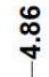

กิ ฺุ
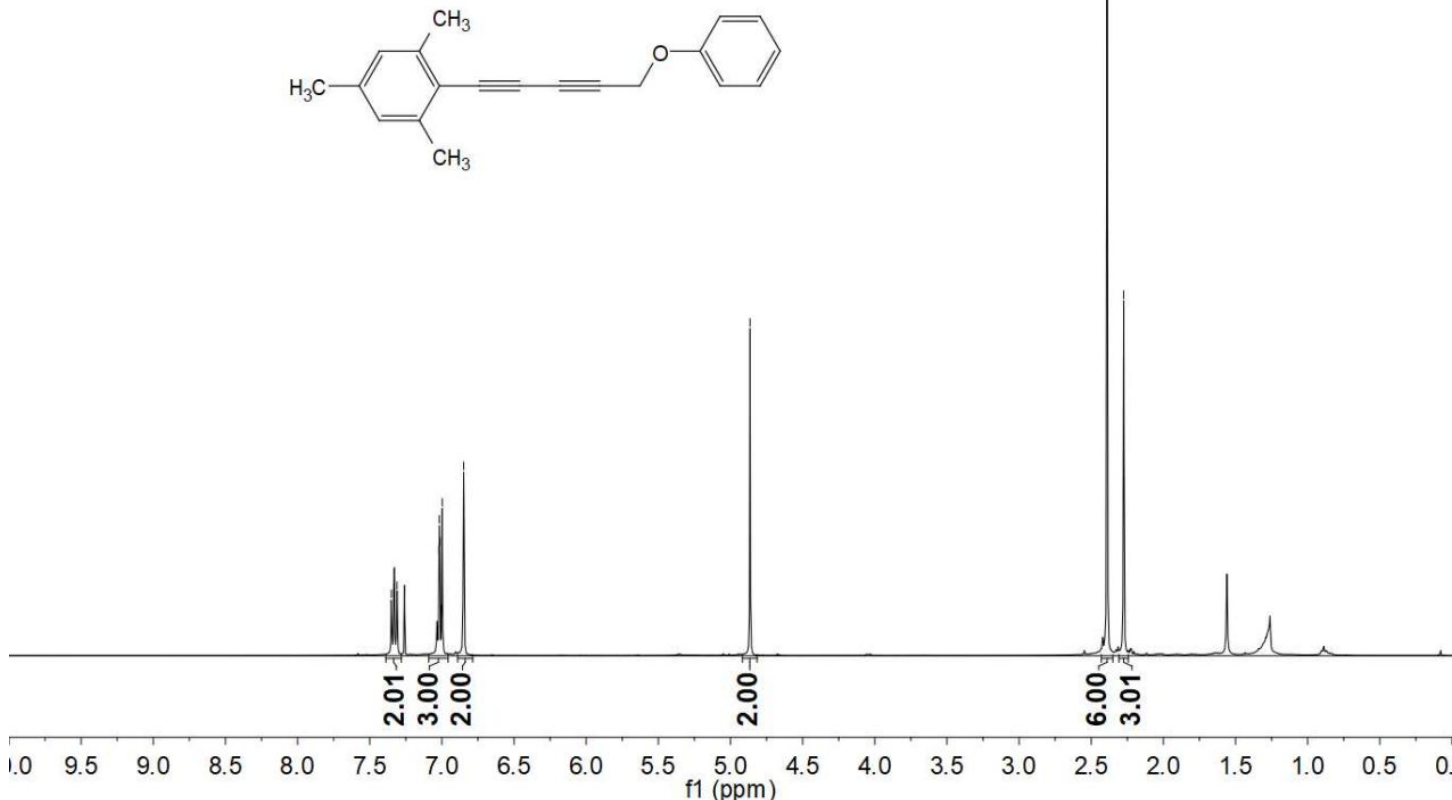

Figure S117. ${ }^{1} \mathrm{H}$ NMR spectroscopy of $4 \mathbf{a a}$.

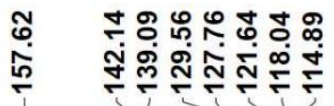

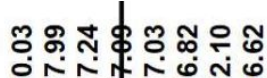

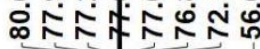

$\exists \circ$

กั่
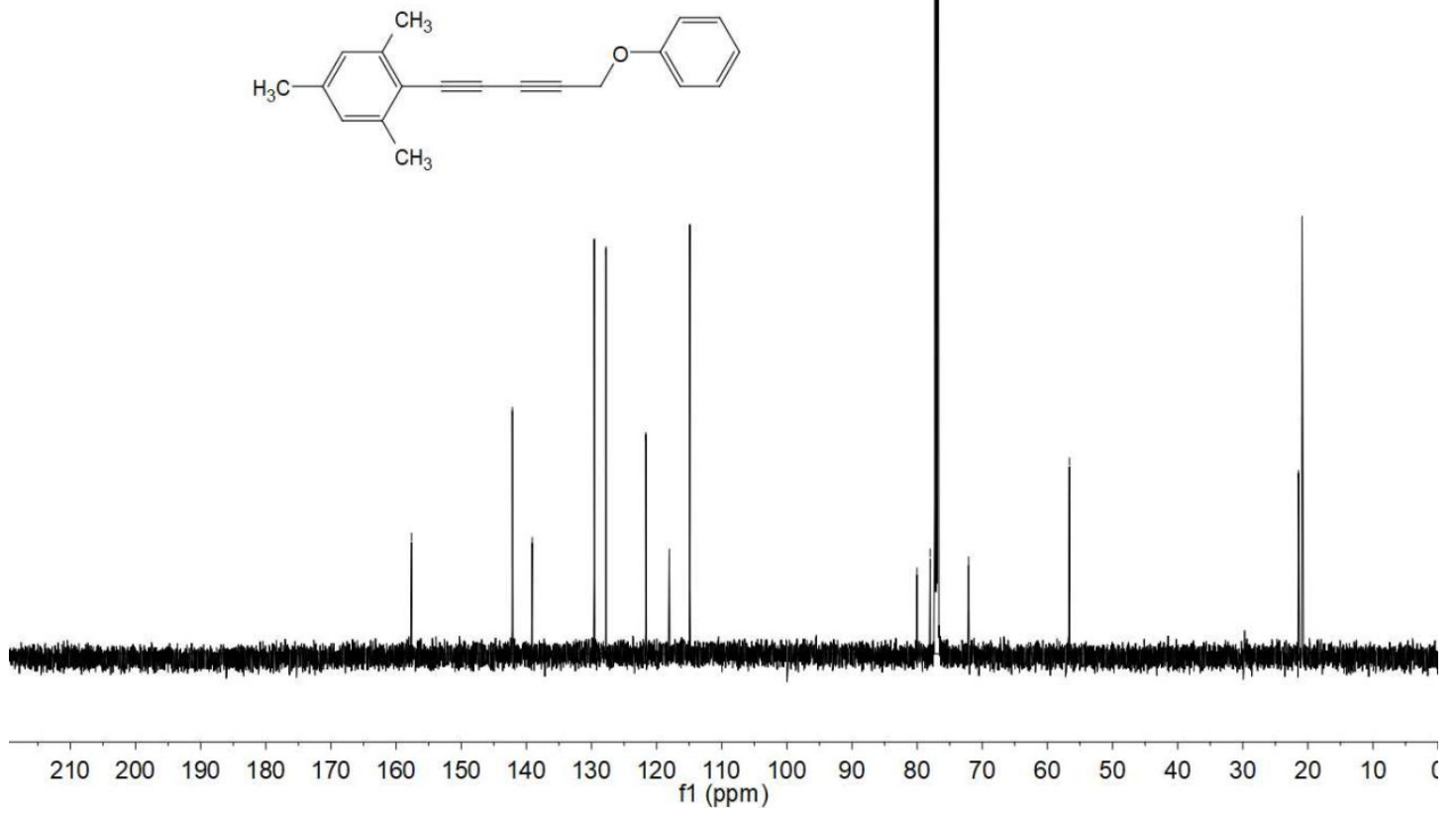

Figure S118. ${ }^{13} \mathrm{C}$ NMR spectroscopy of 4aa. 


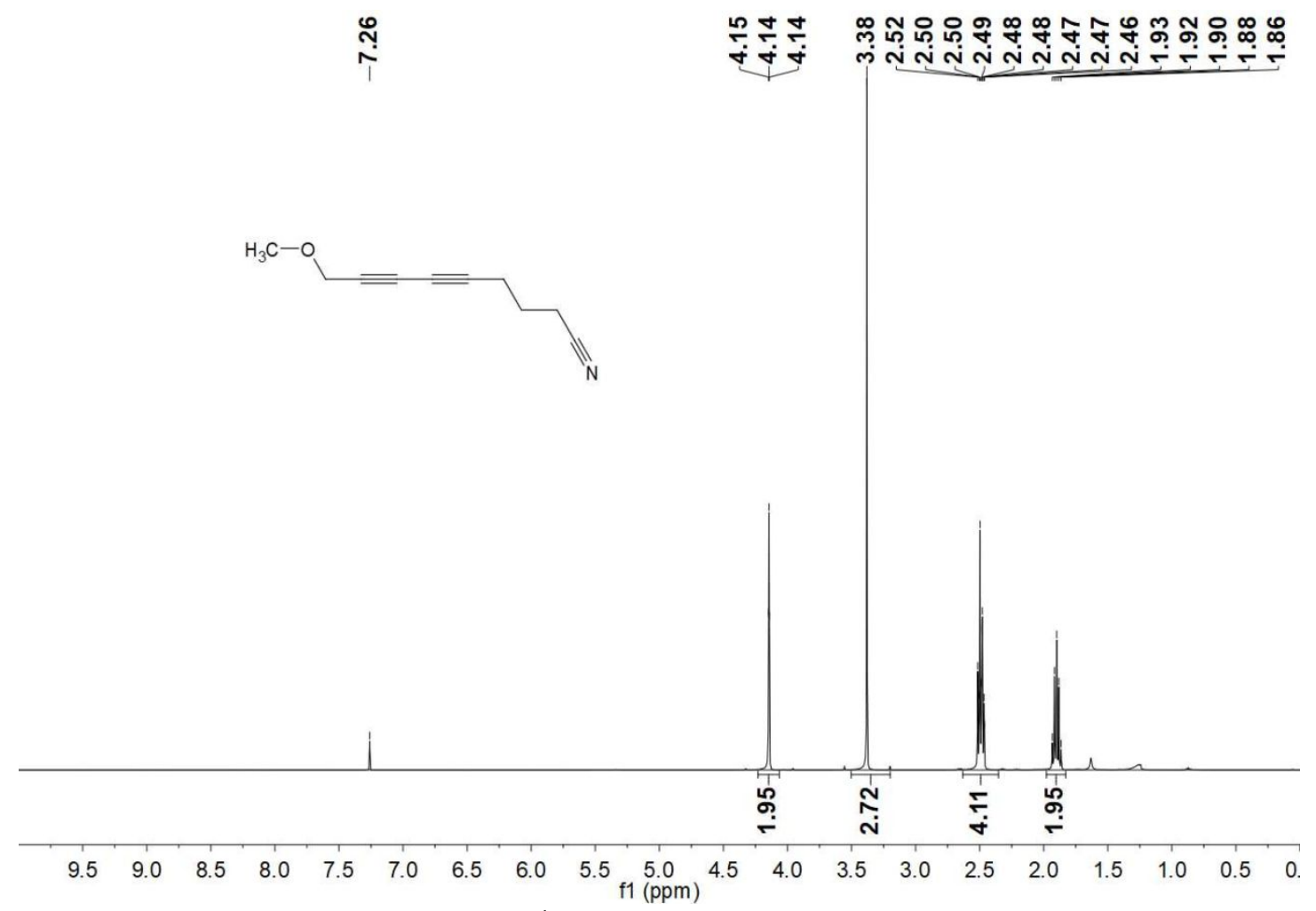

Figure S119. ${ }^{1} \mathrm{H}$ NMR spectroscopy of $4 \mathbf{a b}$.

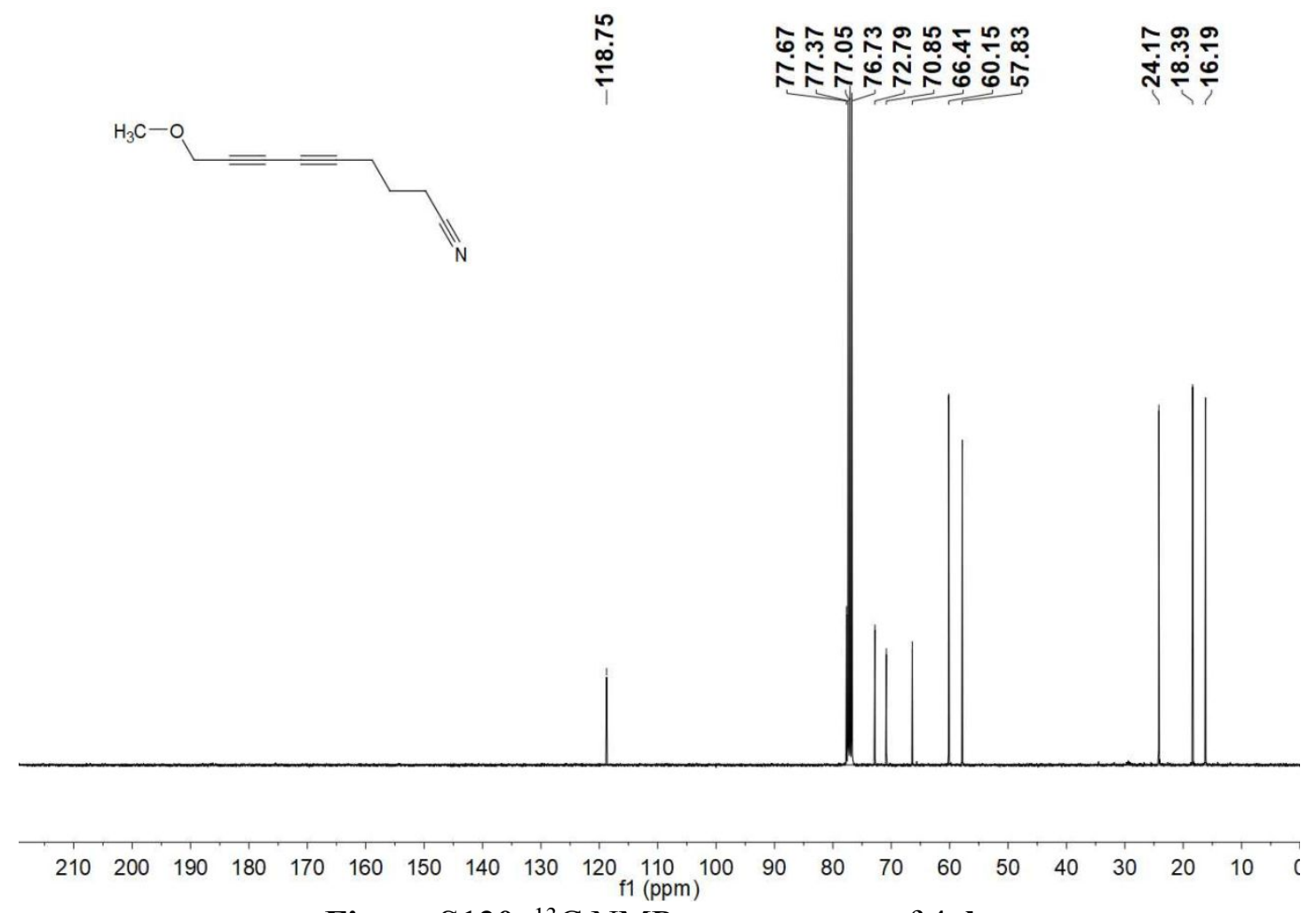

Figure S120. ${ }^{13} \mathrm{C}$ NMR spectroscopy of $4 \mathbf{a b}$. 
సุํำ

HO
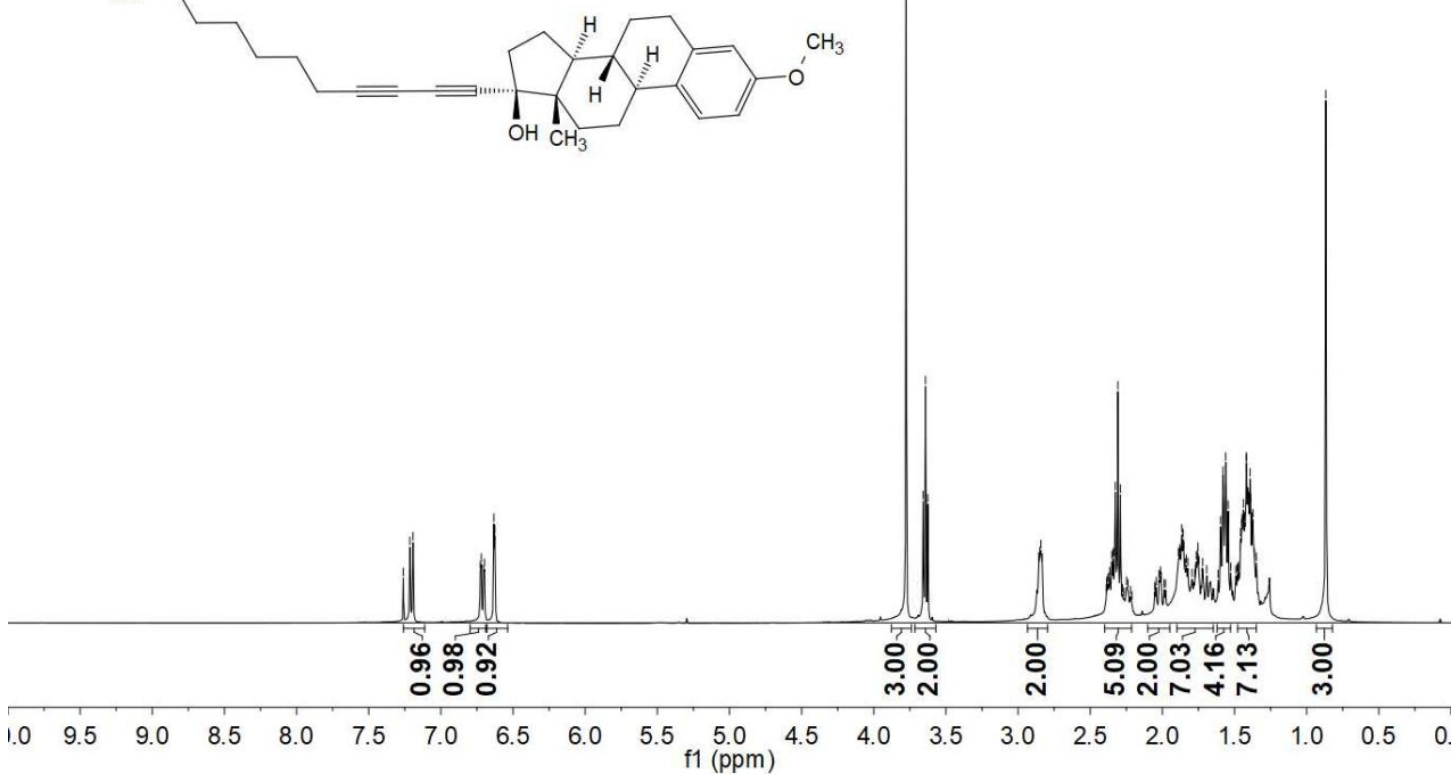

Figure S121. ${ }^{1} \mathrm{H}$ NMR spectroscopy of 4ac

รูํํำ

ผ

HO
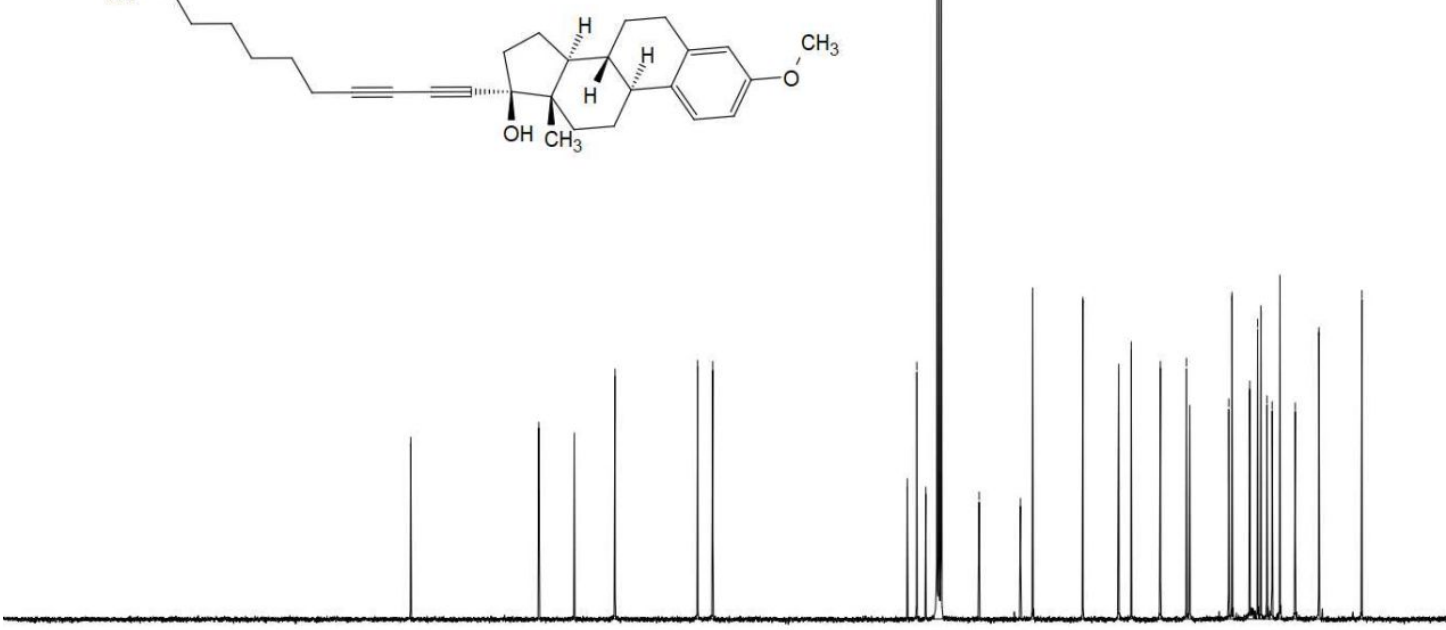

$\begin{array}{llllllllllllllllllll}210 & 200 & 190 & 180 & 170 & 160 & 150 & 140 & 130 & 120 \\ \mathrm{f} 1(\mathrm{ppm}) & 110 & 100 & 90 & 80 & 70 & 60 & 50 & 40 & 30 & 20 & 10 & (\end{array}$

Figure S122. ${ }^{13} \mathrm{C}$ NMR spectroscopy of 4ac. 

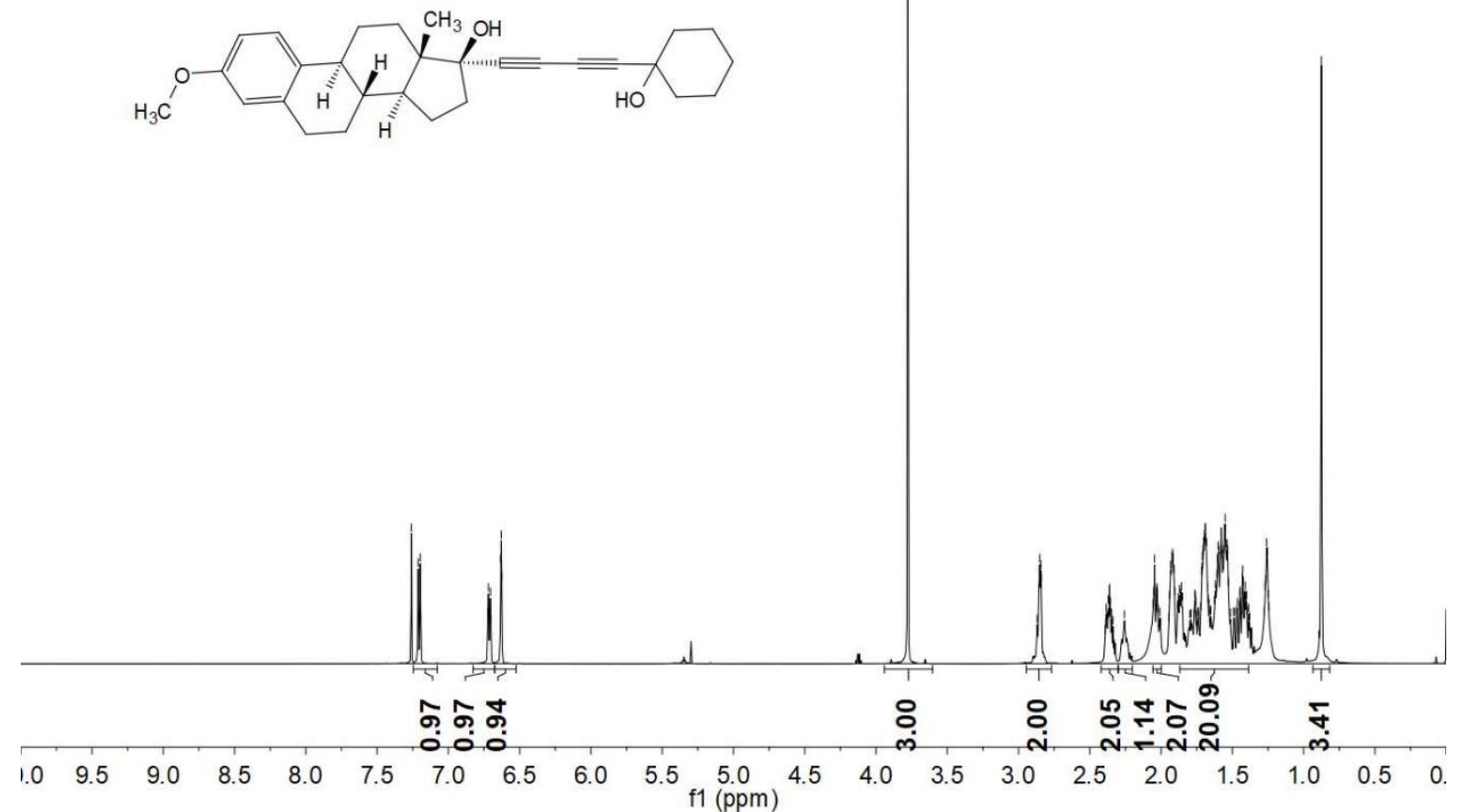

Figure S123. ${ }^{1} \mathrm{H}$ NMR spectroscopy of 4ad.

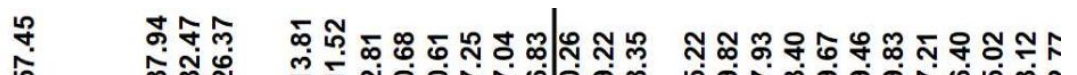

L
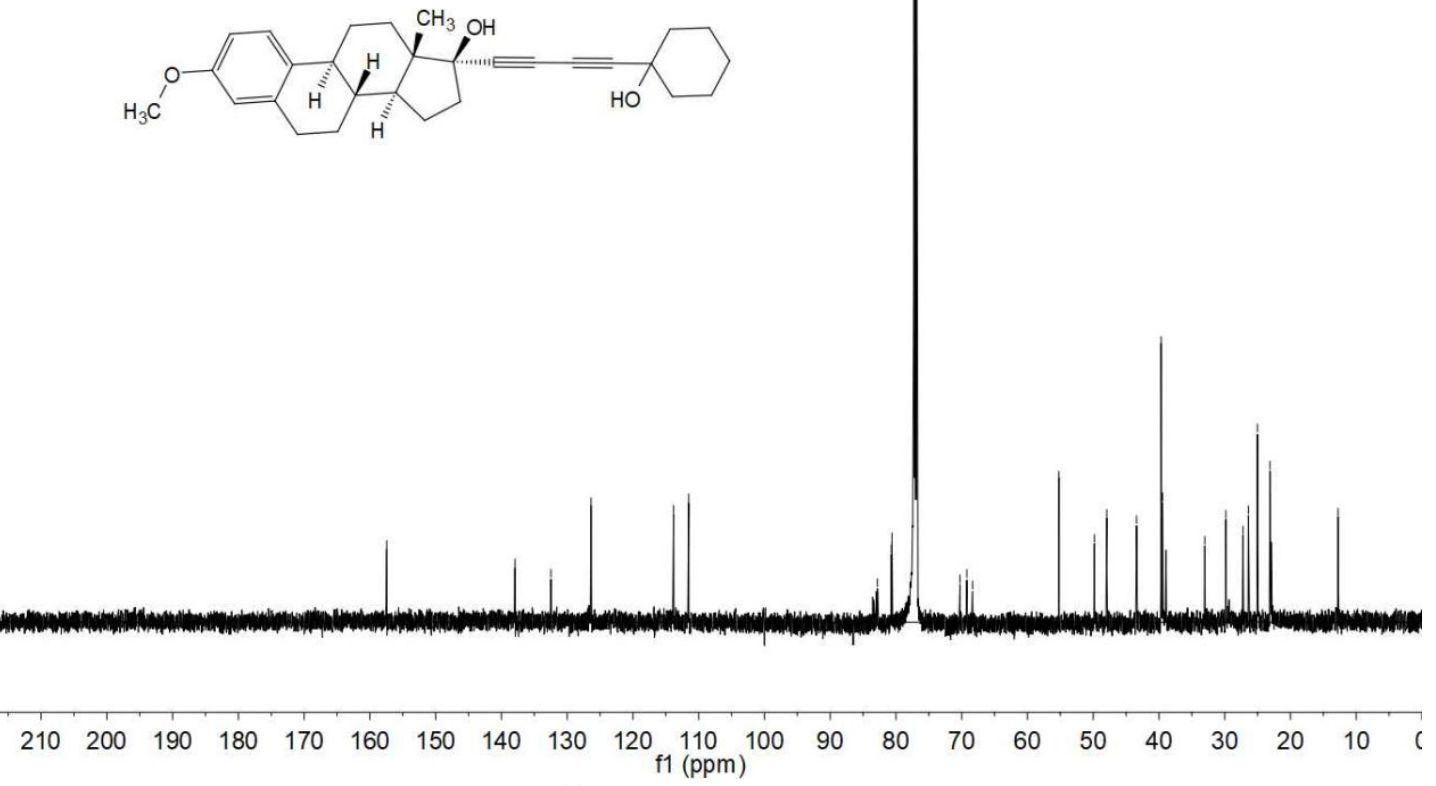

Figure S124. ${ }^{13} \mathrm{C}$ NMR spectroscopy of 4 ad. 

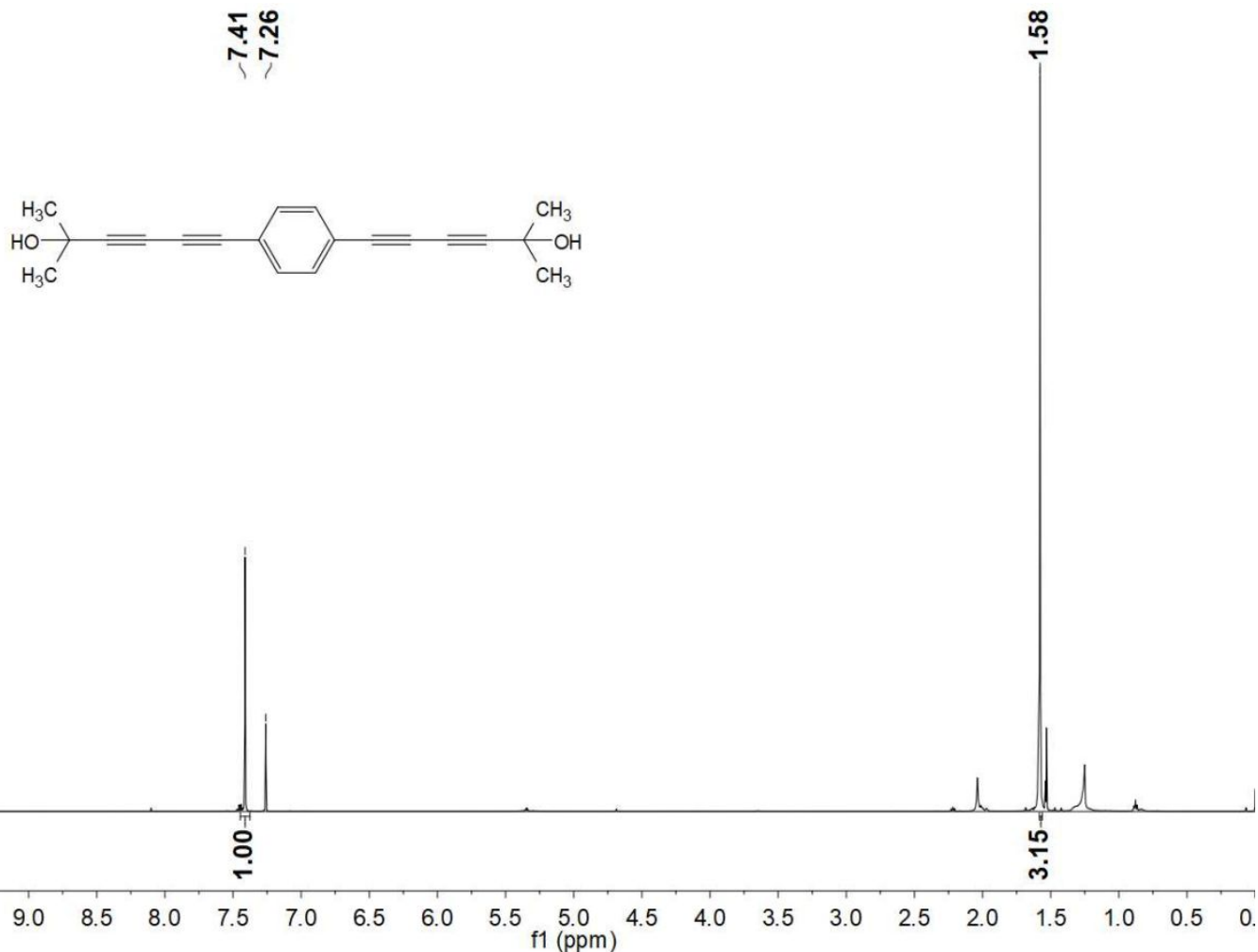

Figure S125. ${ }^{1} \mathrm{H}$ NMR spectroscopy of 4ae.

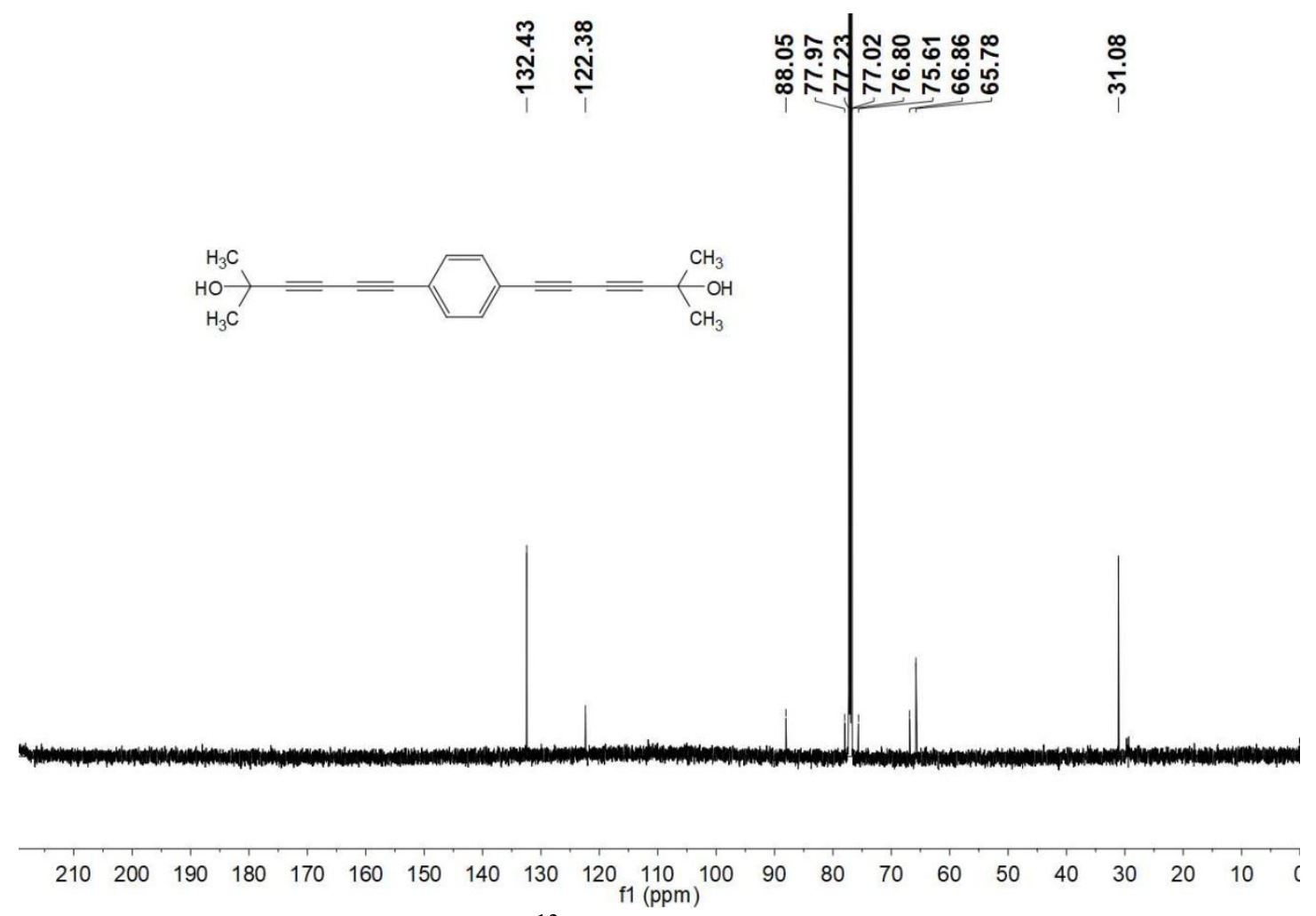

Figure S126. ${ }^{13} \mathrm{C}$ NMR spectroscopy of 4ae. 


\section{References}

1. Kresse, G.; Furthmuller, J., Efficient iterative schemes for ab initio total-energy calculations using a plane-wave basis set. Phys. Rev. B. 1996, 54, 11169.

2. Blöchl, P. E., Projector augmented-wave method. Phys. Rev. B. 1994, 50, 17953.

3. Perdew, J. P.; Burke, K.; Ernzerhof, M., Generalized gradient approximation made simple. Phys. Rev. Lett. 1996, 77, 3865.

4. Teter, M. P.; Payne, M. C.; Allan, D. C., Solution of Schrodinger's equation for large systems. Phys. Rev. B. 1989, 40, 12255.

5. $\quad \mathrm{Xu}, \mathrm{H}$.; Wu, K.; Tian, J.; Zhu, L.; Yao, X., Recyclable $\mathrm{Cu} / \mathrm{C}_{3} \mathrm{~N}_{4}$ composite catalysed homo- and cross-coupling of terminal alkynes under mild conditions. Green Chem. 2018, 20, 793.

6. Singh, M.; Singh, A. S.; Mishra, N.; Agrahari, A. K.; Tiwari, V. K., Benzotriazole as an Efficient Ligand in Cu-Catalyzed Glaser Reaction. ACS Omega 2019, 4, 2418.

7. Liu, D.-X.; Li, F.-L.; Li, H.-X.; Gong, W.-J.; Gao, J.; Lang, J.-P., Efficient and Reusable $\mathrm{CuI} / 1,10$-Phenanthroline-Catalyzed Oxidative Decarboxylative Homocoupling of Arylpropiolic Acids in Aqueous DMF. Eur. J. Org. Chem. 2014, 2014, 4817.

8. Weber, S. M.; Hilt, G., Chemoselective Cobalt(I)-Catalyzed Cyclotrimerization of (Un)Symmetrical 1,3-Butadiynes for the Synthesis of 1,2,4-Regioisomers. Org. Lett. 2019, 21, 4106.

9. Merkul, E.; Urselmann, D.; Müller, T. J. J., Consecutive One-Pot Sonogashira-Glaser Coupling Sequence - Direct Preparation of Symmetrical Diynes by Sequential Pd/Cu Catalysis. Eur. J. Org. Chem. 2011, 2011, 238.

10. Biswas, S.; Mullick, K.; Chen, S.-Y.; Kriz, D. A.; Shakil, M. D.; Kuo, C.-H.; AngelesBoza, A. M.; Rossi, A. R.; Suib, S. L., Mesoporous Copper/Manganese Oxide Catalyzed Coupling of Alkynes: Evidence for Synergistic Cooperative Catalysis. Acs Catal. 2016, 6, 5069.

11. Rzonsowska, M.; Dudziec, B.; Kownacki, I.; Marciniec, B., New protocol for one-pot synthesis of functionalized symmetrical 1,4-dialkyl- or 1,4-diaryl-1,3-diynes. J. Organomet. Chem. 2015, 775, 20.

12. Vilhanová, B.; Václavík, J.; Artiglia, L.; Ranocchiari, M.; Togni, A.; van Bokhoven, J. A., Subnanometer Gold Clusters on Amino-Functionalized Silica: An Efficient Catalyst for the Synthesis of 1,3-Diynes by Oxidative Alkyne Coupling. Acs Catal. 2017, 7, 3414.

13. Tang, S.; Li, L.; Ren, X.; Li, J.; Yang, G.; Li, H.; Yuan, B., Metallomicelle catalyzed aerobic tandem desilylation/Glaser reaction in water. Green Chem. 2019, 21, 2899.

14. Heo, J.-M.; Kim, Y.; Han, S.; Joung, J. F.; Lee, S.-h.; Han, S.; Noh, J.; Kim, J.; Park, S.; Lee, H.; Choi, Y. M.; Jung, Y.-S.; Kim, J.-M., Chromogenic Tubular Polydiacetylenes from Topochemical Polymerization of Self-Assembled Macrocyclic Diacetylenes. Macromolecules 2017, 50, 900 .

15. Li, S. T.; Schnabel, T.; Lysenko, S.; Brandhorst, K.; Tamm, M., Synthesis of unsymmetrical 1,3-diynes via alkyne cross-metathesis. Chem. Commun. 2013, 49, 7189.

16. Liu, Y.; Liu, P.; Gu, N.; Xie, J.; Liu, Y.; Dai, B., Synthesis of Unsymmetrical 1,3-Diynes via $\mathrm{Pd} / \mathrm{Cu}-\mathrm{Catalyzed}$ Cross-Coupling of Terminal Alkynes at Room Temperature. Chin. J. Chem. 2016, 34, 895.

17. Su, L.; Dong, J.; Liu, L.; Sun, M.; Qiu, R.; Zhou, Y.; Yin, S. F., Copper Catalysis for Selective Heterocoupling of Terminal Alkynes. J. Am. Chem. Soc. 2016, 138, 12348.

18. Wang, Y.; Suo, Q.; Han, L.; Guo, L.; Wang, Y.; Li, F., Copper(II)/Palladium(II) catalysed highly selective cross-coupling of terminal alkynes in supercritical carbon dioxide. Tetrahedron 2018, 74, 1918.

19. Fan, X.; Li, N.; Shen, T.; Cui, X.-M.; Lv, H.; Zhu, H.-B.; Guan, Y.-H., A mild CuI- 
catalyzed Glaser-type homo-coupling reaction using $\alpha, \alpha$-dibromo- $\beta$-dicarbonyl compounds as oxidants. Tetrahedron 2014, 70, 256. 
20. Huang, Z.; Shang, R.; Zhang, Z. R.; Tan, X. D.; Xiao, X.; Fu, Y., Copper-catalyzed decarboxylative coupling of alkynyl carboxylates with 1,1-dibromo-1-alkenes. J. Org. Chem. 2013, 78 (9), 4551-7.

21. Chinta, B. S.; Baire, B., A systematic study on the Cadiot-Chodkiewicz cross coupling reaction for the selective and efficient synthesis of hetero-diynes. RSC Adv. 2016, 6 (59), 5444954455 .

22. Li, X.; Xie, X.; Sun, N.; Liu, Y., Gold-Catalyzed Cadiot-Chodkiewicz-type CrossCoupling of Terminal Alkynes with Alkynyl Hypervalent Iodine Reagents: Highly Selective Synthesis of Unsymmetrical 1,3-Diynes. Angew. Chem. Int. Ed. 2017, 56 (24), 6994-6998. 